\title{
STED Microscopy of FRET Pairs
}

\author{
Dissertation \\ for the award of the degree \\ "Doctor rerum naturalium" \\ of the Georg-August-Universität Göttingen
}

within the

International Max Planck Research School

Physics of Biological and Complex Systems

of the

Göttingen Graduate School for Neurosciences, Biophysics, and Molecular Biosciences

\author{
submitted by \\ Maria Loidolt-Krüger \\ from Lutherstadt Wittenberg
}

Göttingen, January 2018 



\section{Thesis Committee Members}

Prof. Dr. Stefan W. Hell (Referee)

Department of NanoBiophotonics,

Max Planck Institute for Biophysical Chemistry, Göttingen

Prof. Dr. Sarah Köster ( $2^{\text {nd }}$ Referee)

Research Group for Nanoscale Imaging of Cellular Dynamics,

Institute for X-ray Physics, Georg-August-Universität Göttingen

Prof. Dr. Helmut Grubmüller

Department of Theoretical and Computational Biophysics,

Max Planck Institute for Biophysical Chemistry, Göttingen

\section{Further Members of the Examination Board}

\section{Dr. Thomas Burg}

Research Group of Biological Micro- and Nanotechnology,

Max Planck Institute for Biophysical Chemistry, Göttingen

PD Dr. Alexander Egner

Department of Optical Nanoscopy,

Laser-Laboratorium Göttingen e.V.

Prof. Dr. Fred S. Wouters

Laboratory for Molecular and Cellular Systems,

Institute for Neuropathology, Georg-August-Universität Göttingen

Date of oral examination: 2018-03-19 



\section{Abstract}

Förster resonance energy transfer (FRET) is a popular tool in life sciences, for example to detect protein-protein interactions and ligand binding, or to construct fluorescent biosensors for metabolites or ions. Obtaining such functional information provided by FRET from diffraction-unlimited images would be advantageous, because, for one thing, the spatial averaging of fluorescence signals could be reduced with a smaller detection volume, and, for another thing, FRET signals from neighboring subdiffraction areas could be distinguished. Thus, the goal of this thesis was to investigate the feasibility of measuring FRET using stimulated emission depletion (STED) microscopy.

Numerical simulations of a single FRET pair under continuous-wave and pulsed STED illumination were performed to study the interplay of FRET and STED photophysics, including the influence of STED intensity and pulse delay.

Organic fluorophores were screened to identify a STED-compatible FRET pair, and the dyes Atto532 and Star635P were chosen for further experiments. Fluorescence lifetime imaging (FLIM) STED microscopy of single FRET pairs bound to dsDNA at defined distances was performed with various STED laser powers and STED pulse delays. Thus measured fluorescence decay curves were compared with simulated ones to confirm the validity of the simplified photophysical model that was established to describe the measurement process. Based on this model, the measurement strategy was devised and the measurement settings were chosen. The spectral detection channels of donor, FRET and acceptor signals differ in spatial resolution; this excludes the use of intensity-based FRET quantification methods. Only the analysis of acceptor fluorescence decay curves can yield diffraction-unlimited FRET information.

Large spectral shifts of the fluorescence emission of both fluorophores were observed in single-molecule measurements. STED imaging showed that emission shifts to shorter wavelengths can decrease the STED efficiency and thus the image resolution. The spectral shifts also change the spectral overlap integral of donor emission and acceptor absorption and thereby the FRET efficiency; the magnitude of this effect was estimated.

In summary, a photophysical model for the interference of STED and FRET was established. Experimental requirements for STED imaging of FRET pairs were identified and a suitable data analysis strategy was proposed. Additionally, photoconversion of organic fluorophores was characterized and its effect on STED and FRET investigated. 


\section{Zusammenfassung}

Der Förster-Resonanzenergietransfer (FRET) ist ein beliebtes Werkzeug der Lebenswissenschaften. Er wird z.B. benutzt um Proteininteraktionen oder das Binden von Liganden zu detektieren, oder um fluoreszente Biosensoren für Stoffwechselprodukte oder Ionen zu konstruieren. Es wäre vorteilhaft, solche funktionellen Informationen, wie man sie durch FRET erhält, aus hochaufgelösten Bildern auslesen zu können. Zum einen würde die räumliche Mittelung der Fluoreszenzsignale durch ein kleineres Detektionsvolumen reduziert werden. Zum anderen könnten FRET-Signale aus dicht beieinander liegenden Zonen, die kleiner als die Beugungsgrenze sind, voneinander unterschieden werden. Daher war es das Ziel dieser Dissertation, die Durchführbarkeit von FRET-Messungen mittels STED-Mikroskopie (STED - Stimulated Emission Depletion) zu prüfen.

Numerische Simulationen eines einzelnen FRET-Paares unter Dauerstrich- und gepulster STED-Beleuchtung wurden durchgeführt, um das Zusammenspiel der photophysikalischen Prozesse FRET und STED zu untersuchen, den Einfluss der STED-Laserintensität und der Pulsverzögerung mit eingeschlossen.

Organische Fluorophore wurden getestet, um ein mit STED kompatibles FRET-Paar zu identifizieren; die Farbstoffe Atto532 und Star635P wurden für die weiteren Experimente ausgewählt. Fluoreszenzlebensdauer-STED-Mikroskopie einzelner FRET-Paare, die in bestimmten Abständen an doppelsträngiger DNA befestigt waren, wurde mit verschiedenen STED-Laserleistungen und STED-Pulsverzögerungen durchgeführt. Auf diese Weise gemessene Fluoreszenzlebensdauerhistogramme wurden mit simulierten Histogrammen verglichen, um die Gültigkeit des vereinfachten photophysikalischen Modells zu testen, welches entwickelt wurde, um den Messprozess zu beschreiben. Basierend auf diesem Modell wurde die Messstrategie entworfen und die Messeinstellungen gewählt. Die spektralen Detektionskanäle des Donor-, FRET- und Akzeptorsignals unterscheiden sich in der räumlichen Auflösung, dies schließt die Verwendung intensitätsbasierter FRET-Quantifizierungsmethoden aus. Nur die Analyse der Fluoreszenzlebensdauerhistogramme des Akzeptors kann beugungsunbegrenzte FRETInformationen liefern.

Große spektrale Verschiebungen der Fluoreszenz beider Fluorophore wurden in Einzelmolekülmessungen beobachtet. In STED-Bildern zeigte sich, dass eine Verschiebung der Emission hin zu kürzeren Wellenlängen die STED-Effizienz verringern kann und damit auch die Auflösung der Bilder. Die spektralen Verschiebungen ändern auch das spektrale Überlappintegral von Donoremission und Akzeptorabsorption und dadurch die FRET-Effizienz; die Größe dieses Effekts wurde abgeschätzt. 
Kurz zusammengefasst wurde ein photophysikalisches Modell für die Interferenz von STED und FRET eingeführt. Experimentelle Voraussetzungen für die STED-Mikroskopie von FRET-Paaren wurden identifiziert und eine adäquate Datenanalysestrategie wurde vorgeschlagen. Zusätzlich wurde die Photoumwandlung organischer Fluorophore charakterisiert und deren Auswirkung auf STED und FRET untersucht. 



\section{Contents}

1 Introduction 1

I Theoretical background 3

2 Theory of fluorescence $\quad 5$

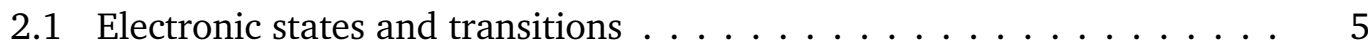

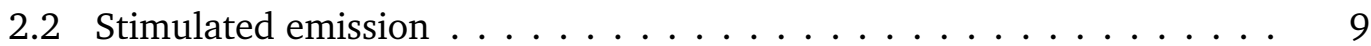

3 Photochemistry of organic fluorophores $\quad 11$

3.1 Fluorescence quenching . . . . . . . . . . . . . . . . . 11

3.2 Transient dark states . . . . . . . . . . . . . . . . . . . . . 12

3.3 Spectral diffusion . . . . . . . . . . . . . . . . . . . . . 12

3.4 Photobleaching . . . . . . . . . . . . . . . . 13

3.5 Photochromism / Photoswitching . . . . . . . . . . . . . 14

3.6 Photoactivation . . . . . . . . . . . . . . . . . . . . 14

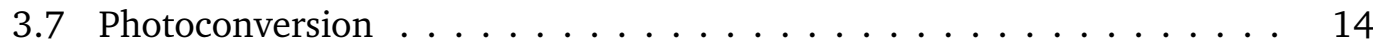

4 Theory of light microscopy 15

4.1 Image formation in the light microscope . . . . . . . . . . . . . . 15

4.2 Resolution and the diffraction limit . . . . . . . . . . . . . 17

4.3 The fluorescence microscope . . . . . . . . . . . . . . . 18

4.4 The confocal microscope . . . . . . . . . . . . . . . . . . . 19

5 Nanoscopy 21

5.1 Coordinate-targeted nanoscopy . . . . . . . . . . . . . . . 21

5.1.1 Stimulated emission depletion nanoscopy . . . . . . . . . . . . . . . 21

5.2 Single molecule localization microscopy . . . . . . . . . . . . . . . . . 24

5.3 Nanoscopy with minimal emission fluxes . . . . . . . . . . . . . . . 24

6 Förster resonance energy transfer $\quad 25$

6.1 Theory of FRET . . . . . . . . . . . . . . . . . . . . . 25

6.2 Quantification of FRET _ . . . . . . . . . . . . . . . 27

6.2.1 Fluorescence intensity-based methods . . . . . . . . . . . . . . 27

6.2.2 Fluorescence lifetime-based methods . . . . . . . . . . . . . . . 29

6.2.3 Fluorescence anisotropy-based methods . . . . . . . . . . . . . 30 
7 Brief literature review: Biological applications 33

7.1 Biological applications of STED nanoscopy $\ldots \ldots \ldots \ldots$

7.2 Biological applications of FRET imaging $\ldots \ldots \ldots \ldots$

8 Motivation: Combining FRET and STED for functional imaging 37

9 Brief literature review: Use of FRET for nanoscopy methods 39

9.1 FRET induced blinking to facilitate SMLM . . . . . . . . . . . . . 39

9.2 Enhancing resolution with saturated FRET microscopy . . . . . . . . . . 40

9.3 FRET and SMLM provide complementary information . . . . . . . . . . . 41

9.4 Extracting FRET efficiency from superresolution images . . . . . . . . . . 41

$\begin{array}{ll}\text { III Simulations } & 43\end{array}$

10 Combining FRET with CW-STED 47

10.1 System of rate equations for FRET with CW-STED . . . . . . . . . . . 47

10.2 Changing the FRET rate under CW-STED light $\ldots \ldots \ldots$. . . . . . 50

10.3 Changing the CW-STED intensity at constant FRET rate . . . . . . . . 52

11 Combining FRET with pulsed STED $\mathbf{5 5}$

11.1 System of rate equations for pulsed STED . . . . . . . . . . . 55

11.2 Changing the FRET rate under pulsed STED light $\ldots \ldots \ldots 7$

11.3 Changing STED intensity at constant FRET rate . . . . . . . . . . . . 57

11.4 Changing STED delay at constant FRET rate . . . . . . . . . . . . 59

11.5 Evolution of the acceptor's PSF over time $\ldots \ldots \ldots 60$

11.6 Donor with a short lifetime . . . . . . . . . . . . . 63

$\begin{array}{ll}12 \text { Summary and discussion of the simulations } & 67\end{array}$

$\begin{array}{ll}\text { IV Experimental Implementation } & 71\end{array}$

13 Microscope setup 73

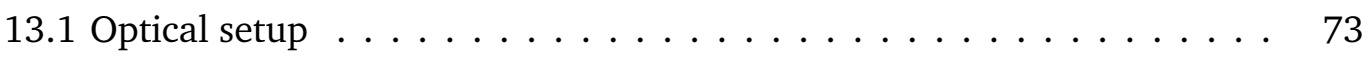

13.2 Electronic control . . . . . . . . . . . . . . . . . 79

14 Data acquisition $\quad 81$

14.1 Measurement acquisition software . . . . . . . . . . . 81

14.2 Single molecule measurement setup and workflow . . . . . . . . . . 82

15 Fluorescently labeled DNA constructs 85

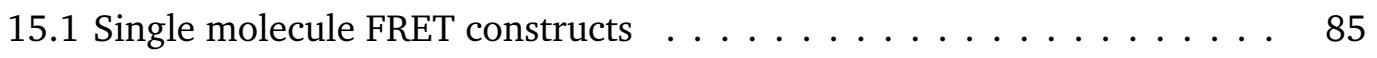

15.2 Constructs with single fluorophores $\ldots \ldots \ldots \ldots$. . . . . . 86 
16 Sample Preparation $\quad 87$

16.1 Buffers . . . . . . . . . . . . . . . . . . . . 87

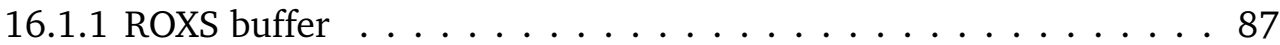

16.1.2 DNA storage buffer . . . . . . . . . . . . . . 87

16.2 Dye solutions . . . . . . . . . . . . . . . . . . . 887

16.3 Gold bead alignment samples . . . . . . . . . . . . . . . 88

16.4 Immunostaining of fixed cells $\ldots \ldots \ldots$. . . . . . . . . 88

16.5 Cleaning of coverslips and microscope slides . . . . . . . . . . . . 89

16.6 Immobilization of labeled DNA _ . . . . . . . . . . . . . . . . . 89

$\begin{array}{ll}\text { V Experimental Results } & 91\end{array}$

17 Selection of a STED-compatible dye pair for FRET experiments 93

17.1 Donor fluorophores . . . . . . . . . . . . . . . . . . . 93

17.2 Acceptor fluorophores . . . . . . . . . . . . . . . . . . . . 96

17.3 Summary and discussion $\ldots \ldots \ldots \ldots$. . . . . . . . . . 98

18 Comparison of measured and simulated decay curves 99

18.1 Summary and discussion . . . . . . . . . . . . . . . . . 104

19 Differing spatial resolution $\quad 105$

19.1 Summary and discussion . . . . . . . . . . . . . . . . 110

20 Finding a measure for the FRET efficiency compatible with STED 111

20.1 Obstacles to using established FRET measures on STED data . . . . . . . . 111

20.2 Normalizing to the acceptor emission . . . . . . . . . . . . . . . 112

20.2.1 Combining intensity ratio and photon arrival time . . . . . . . 113

20.3 Analysis of acceptor decay curves . . . . . . . . . . . . . . . 115

21 Spectral shifting of fluorophores $\quad 119$

21.1 Confocal measurements of spectral shifts . . . . . . . . . . . . . . . 119

21.2 STED measurements of spectral shifts . . . . . . . . . . . . . . . 129

21.3 Summary and discussion . . . . . . . . . . . . . . . . . . 135

21.3.1 Spectral diffusion . . . . . . . . . . . . . . . . . 135

21.3.2 Photoconversion . . . . . . . . . . . . . . . . . 136

21.3.3 Implications of blueing for STED imaging . . . . . . . . . . . 137

22 Effect of spectral shifts on the FRET efficiency 139

22.1 Overlap integral changes . . . . . . . . . . . . . . . . . . . . . 139

22.2 Spectral changes in single molecule FRET time traces . . . . . . . . . . 140

22.2.1 Summary and discussion . . . . . . . . . . . . . . . . . 148 
25 Combining FRET with other nanoscopy techniques 155

25.1 Combining with single molecule localization microscopy . . . . . . . . 155

25.2 Combining with coordinate-targeted nanoscopy other than STED . . . . 158

26 Comparison of FRET and MINFLUX

VII Conclusions and outlook

27 Supplementary theory 175

27.1 Theory of acousto-optical modulators . . . . . . . . . . . . . . . 175

27.2 Theory of acousto-optical tunable filters . . . . . . . . . . . 175

$\begin{array}{ll}28 \text { Supplementary figures } & 179\end{array}$

29 MATLAB codes $\quad 187$

$\begin{array}{ll}30 \text { Python codes } & 201\end{array}$

$\begin{array}{ll}31 \text { AOTF control scripts } & 207\end{array}$

$\begin{array}{ll}32 \text { Image acquisition parameters } & 211\end{array}$

32.1 Green and red dye screening parameters . . . . . . . . . . . . . . 211

32.2 Scan parameters for single molecule photoconversion and single pair

FRET experiments . . . . . . . . . . . . . . . . . . 213

$\begin{array}{lr}\text { Bibliography } & 215\end{array}$ 


\section{Acknowledgement}

At this point, I would like to thank all the people who accompanied and supported me during my Ph.D. time. First and foremost, I thank Prof. Stefan W. Hell for the opportunity to work on this exciting and challenging project, for creating an outstanding research environment and for letting me develop my own ideas freely. I would also like to thank my thesis advisory committee members Prof. Sarah Köster and Prof. Helmut Grubmüller for their insightful comments and kind advice.

My thanks go to Dr. Volker Westphal, for his guidance, instructive discussions and expert advice not only on matters of optics and electronics, as well as his constructive comments on this dissertation. I am also grateful to Dr. Franziska R. Winter for her mentoring during my Diploma thesis work and for teaching me how to build an optical setup.

I could not have pursued this project without the expert advice of my colleagues, many thanks to them (in no particular order).

Dr. Jan Keller-Findeisen, for his patient help with programming in MATLAB and for helpful comments on this dissertation.

Dr. Marcel Leutenegger, for sharing his profound theoretical and practical knowledge of TCSPC and the inner workings of $\mathrm{B} \& \mathrm{H}$ cards and for fruitful discussions about fluorophore photoconversion, as well as his detailed comments on this dissertation.

Jaydev Jethwa for knowing where to find everything, for holding everything in order and for his invaluable practical help with any kind of problem with electronics, lasers or any other device.

Dr. Marco Roose for his help with IT issues of any kind and for supplying delicious honey.

Björn Thiel, for aiding me with Imspector, Python and interfacing both programs.

Dr. Alexey N. Butkevich, for supplying a variety of great fluorophores and for kindly explaining their behavior to me.

Dr. Klaus Gwosch and Dr. Yvan Eilers for kindly teaching me how to prepare single molecule samples and for instructive discussions about MINFLUX. 
Michael Weber, for helpful discussions and literature tips, especially regarding fluorophores, buffers and DNA constructs.

Ellen Rothermel for preparing very many perfectly stained fixed cell samples for fluorophore testing.

Stefanie Kruse and Sarah Leondarakis for their advice on all kinds of organizational matters.

The team of the mechanics workshop for capably and readily providing me with adapters, fitting parts and adjustments of components for my setup.

The team of the electronics workshop for expertly creating various electronic components for my setup.

I am grateful to have had such wonderful office mates - Franziska R. Winter, Elke Hebisch, Yvan Eilers, Jasmin Pape and Robert Braunschweig - who created a comfortable working atmosphere and were always there for fun as well as serious conversations. I will also miss many stimulating lunch time discussions with Marcel Leutenegger, Michael Weber, Steffen J. Sahl and Roman Schmidt. Warm thanks also to all the great people in the NanoBiophotonics and Grubmüller department whom I have not mentioned yet for the good times inside and outside the institute.

Heartfelt thanks go to my husband Matthias H. Krüger and my family who continuously supported me, always had an open ear for my troubles and grievances and if necessary reminded me that it was indeed my choice to pursue a Ph.D..

Last but not least, I am grateful to the Max Planck Society for funding me throughout my time at the MPIbpc. 


\section{Glossary of symbols and acronyms}

A acceptor

a.u. arbitrary units

AOBS acousto-optical beam splitter

AOM acousto-optical modulator

AOTF acousto-optical tunable filter

APD avalanche photo diode

CW continuous wave

D donor

FCS fluorescence correlation spectroscopy

FLIM fluorescence lifetime imaging

FOV field of view

FPGA field-programmable gate array

FRET Förster resonance energy transfer

FSK frequency shift keying

FWHM full width at half maximum

GSD ground state depletion microscopy

HyperSTED multicolour STED set-up with a hyperspectral detection

IC internal conversion

ISC intersystem crossing

MPIbpc Max Planck Institute for Biophysical Chemistry

NA numerical aperture

NIM nuclear instrumentation module

PAINT point accumulation for imaging in nanoscale topography

PALM photoactivated localization microscopy

PBS phosphate-buffered saline

PSF point spread function

QS quadscanner

QY quantum yield

RESOLFT reversible saturable/switchable optical fluorescence transitions

ROI region of interest

RSFP reversibly photoswitchable fluorescent protein 
SC supercontinuum

s.d. standard deviation

SMLM single-molecule localization microscopy

SNR signal-to-noise ratio

SOFI super-resolution optical fluctuation imaging

STED stimulated emission depletion

STORM stochastic optical reconstruction microscopy

TCSPC time-correlated single photon counting

TIRF total internal reflection fluorescence (microscopy)

TL tube lens 


\section{Introduction}

A picture is worth a thousand words.

\section{— unknown}

The light microscope is a very important tool in life sciences, because it allows the minimally invasive, nondestructive, 3D imaging of live cells and tissues. This enables the observation of cellular functions in their native state. However, images often suffer from a low contrast, which is due to the small, uniform refractive index of biological material. Therefore, distinguishing particular structures can be difficult.

An major enhancement of light microscopy is the use of fluorescent markers, which can specifically label an object of interest. Thus, a fluorescence microscope can visualize specific structures with a high contrast in living cells and tissues. Fluorescence microscopes are often implemented as confocal laser scanning microscopes, because background fluorescence from out of focus layers can be suppressed this way and 3D image stacks can be acquired.

The spatial resolution of a light microscope is limited by diffraction to about half the wavelength of the utilized light. This is about 180nm in the focus plane and 500nm along the optical axis for 488nm light [118]. Yet proteins have a size of approximately $10 \mathrm{~nm}$. So many processes in cells which are driven by protein interactions take place in subdiffraction volumes. Electron microscopy uses wavelengths that are much shorter than visible light and can therefore reach atomic resolution. However, samples have to be flash frozen or embedded in resin, thinly sliced and placed in vacuum. Thus the direct observation of processes in live cells is not possible. Atomic force microscopy also reaches atomic resolution and operates at room temperature. Yet it can by design only probe surfaces.

Fortunately, the diffraction limit can be overcome in fluorescence microscopy by transiently placing a subset of markers in a dark state. Thus, adjacent fluorophores can be detected sequentially to assemble a diffraction unlimited image. This can be implemented in a coordinate-targeted manner (STED, RESOLFT) with raster scanning or in a coordinate-stochastic manner (PALM, STORM) with widefield imaging. Thus, the visualization of structures inside cells with a lateral resolution down to about $35 \mathrm{~nm}$ and an axial resolution of about 90nm was enabled with STED nanoscopy [93]. 
Often functional information is required in addition to structural data in order to understand the inner workings of cells. That is, one does not only need to know the location and form of cellular organelles and structures such as mitochondria, the endoplasmic reticulum or the microtubule network. One also wants to observe events that constitute function, such as enzymatic substrate cleavage, or ligand binding and protein phosphorylation in signal transduction. Additionally, one needs to monitor the local concentrations of metabolites, ions, oxygen, and so on.

FRET is the transfer of energy from one excited molecule to another ground state molecule via dipole-dipole interaction. It occurs if the two fluorophores, whose spectra overlap, are closer together than 10nm and are oriented roughly parallel to each other. Due to its great sensitivity to distance, FRET has been established as a valuable tool in life sciences to provide functional information about protein interactions, and a lot more.

It would be advantageous to gather functional information provided by FRET from diffraction-unlimited images. On the one hand, one could monitor processes taking place in separate but adjacent subdiffraction areas, e.g. at synaptic vesicles. On the other hand, the spatial averaging of the fluorescence signal collected from an observation volume which contains many markers could be reduced. This would increase the dynamic range of the fluorescence measurement. As a combination of FRET with a nanoscopy technique has not yet been demonstrated, the goal of this thesis is to investigate the feasibility of extracting information about FRET from superresolved images acquired with STED nanoscopy.

This thesis is structured as follows. The first part provides background information about the theory of fluorescence, of photochemistry, of light microscopy and nanoscopy techniques and of FRET. In the second part, biological applications of STED and FRET are briefly reviewed to motivate in which areas a combination of both techniques would be advantageous. Reports about the exploitation of FRET to facilitate nanoscopy techniques and about its use complementary to nanoscopy are briefly reviewed. Simulations of the interplay of STED and FRET are presented in the third part to illustrate the basic photophysics involved. The microscope setup and related software that were used in the experimental realization of STED on FRET pairs as well as the sample preparation are described in the fourth part. The fifth part reports and discusses the experimental results obtained from measurements of single fluorophores and single FRET pairs. Further discussions concerning the use of homo-FRET and the determination of the concentration and stoichiometry of labeled species follow in the sixth part. This part also considers ways to combine FRET with nanoscopy techniques other than STED and compares FRET to the new MINFLUX method. Finally, the conclusions are presented in the seventh part, followed by impulses for further research. 
Part I

Theoretical background 



\section{Theory of fluorescence}

Fluorescence is the ability of some molecules to absorb light and shortly thereafter re-emit light of a different color. Fluorescent molecules, called fluorophores, are characterized by several properties, among them:

- excitation and emission spectra

- molar absorption coefficient

- quantum yield

- excited-state lifetime

In this chapter, the physical origins of these properties will be explained. A detailed treatise can be found in [126]. Moreover, stimulated emission will be introduced, which is at the heart of stimulated emission depletion (STED) microscopy, that will be described in chapter 5.1.1.

Further fluorophore characteristics which are important in practical applications are the fluorophore's photostability, its chemical stability and solubility in aqueous media and its quenching behavior. These features will be outlined in chapter 3 .

\subsection{Electronic states and transitions}

The atoms which make up a molecule are connected by chemical bonds formed by overlapping atomic orbitals. Depending on the electronic orbitals involved, one discriminates $\sigma$ bonds and $\pi$ bonds. Non-bonding electrons are located in $n$ orbitals. Fluorophores contain conjugated systems which consist of alternating single and double carbon-carbon bonds. Such systems originate from overlapping $\pi$ orbitals, independent of the $\sigma$ bonds. Within a conjugated system the electrons participating in the $p i$ orbitals are delocalized. The energy gap between the highest occupied and the lowest unoccupied molecular orbital is relatively small in conjugated systems; it is in the same range as the photon energy of visible light. Due to this, absorption of visible light by molecules and fluorescence are possible. ${ }^{1}$

\footnotetext{
${ }^{1}$ Fluorescence emission due to transitions between atomic orbitals is also possible.
} 
At room temperature, molecules are in their least energetic electronic state, the ground state. Upon absorption of a photon, a molecule gets promoted to an excited state. That means that in most cases either a $\pi$ electron or a non-bonding electron is transferred to an antibonding $\pi^{*}$ orbital. In the latter case, a charge transfer within the molecule is induced.

Classically, the molecule can be viewed as an oscillating dipole, in which the photon's frequency matches a resonance frequency. Quantum mechanically, the transition between ground and excited state is characterized by a transition moment which represents a transient dipole moment resulting from a momentary displacement of charges (Franck-Condon principle). An excellent quantum mechanical description of the processes explained below can be found in chapter 3 of [9]. In a semi-classical description, a photon gets absorbed if it hits the absorption cross-section $\sigma(\lambda)$ of the molecule ${ }^{2}$ and if its energy matches the energy gap $\Delta \mathrm{E}$ between the molecule's ground and excited state, as stated by the Planck-Einstein relation:

$$
\Delta E=h v=\frac{h c}{\lambda}
$$

where $h$ denotes the Planck constant.

A molecule can also be excited by multiple photons if their energies add up to match the energy gap between ground and excited state. The photons have to arrive within about $10^{-16}$ s to be absorbed via short-lived virtual states [9]. In the most common case, two photons with double the wavelength for one-photon absorption are used for excitation. Two-photon excitation can occur accidentally during STED illumination, see chapter 17. But it can also be very useful in practical applications because far-red wavelengths are scattered less and penetrate deeper into biological tissue, where they also excite less autofluorescence. Additionally, due to the high intensities required, the effective focal volume is smaller compared to one-photon excitation at the same wavelength. Compared to one-photon excitation at half the wavelength, the focal volume is larger.

The Franck-Condon principle states that electronic transitions are most probable when the induced changes in the position of the nuclei of the molecule and surrounding solvent molecules are minimal. Thus, it can explain the shape of the absorption and emission spectra of a fluorophore. Nuclear motion takes place on a time scale of $10^{-12}$ to $10^{-10} \mathrm{~s}$, in comparison electrons change their conformation in femtoseconds, i.e. instantaneously in the Born-Oppenheimer approximation. After absorption of a photon, which takes $10^{-15} \mathrm{~s}$, the electrons adapt a non-equilibrium distribution with respect to

\footnotetext{
${ }^{2}$ The molar absorption coefficient $\varepsilon_{\mathrm{A}}(\lambda)$ also describes the ability of the molecular species to absorb light of a certain wavelength. It is commonly given in units of $\left[\frac{\mathrm{L}}{\mathrm{mol} \cdot \mathrm{cm}}\right]$, and is related to the molecular absorption cross-section $\sigma(\lambda)$, given in units of $\mathrm{cm}^{2}$, as follows: $\varepsilon_{\mathrm{A}}(\lambda)=\frac{N_{\mathrm{A}} \sigma(\lambda)}{10^{3} \cdot \ln 10}$.
} 
the nuclei. This induces vibrations of the nuclei, which arrange themselves in a new equilibrium geometry. The transition to the lowest vibrational mode takes $10^{-13}$ to $10^{-11} \mathrm{~s}$. The absorption and emission bands, which can be understood as transition probability distributions, are illustrated in Fig.2.1.

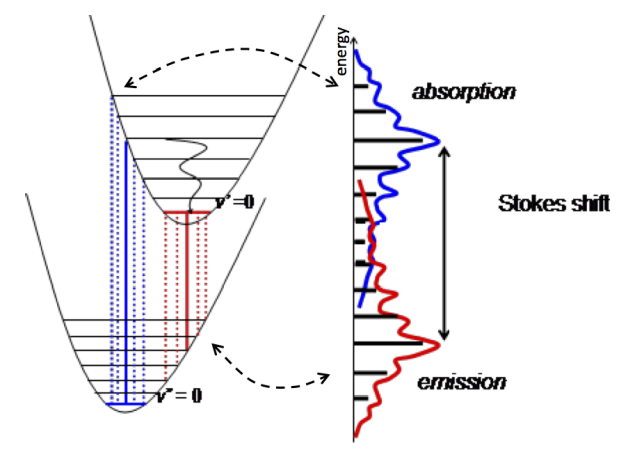

Fig. 2.1: Instantaneous (vertical) transitions, according to the Franck-Condon principle, between different vibrational levels of ground and excited state (potential energy curves of nuclear configurations), give rise to the shape of absorption and emission spectra of fluorophores. Taken from [95].

As one can see in Fig.2.1, the maximum of the emission spectrum is located at a lower energy, corresponding to a longer wavelength, than the absorption maximum. This shift is called the Stokes shift. It is due to the energy loss during the non-radiative vibrational relaxation to the lowest vibrational mode of the excited electronic state. This wavelength shift is of great practical use for fluorescence microscopy, as will be explained in section 4.3 .

The absorption and emission spectra of typically used fluorescent organic dyes do not show discrete bands, as Fig.2.1 suggests. Instead, they are usually smooth curves as shown exemplarily in Fig.2.2. This is because at room temperature in solution an almost continuous set of vibrational modes in each electronic state can be addressed. ${ }^{3}$

The emission spectrum is typically independent of the excitation wavelength, because upon excitation to a higher electronic state, internal conversion (IC) followed by vibrational relaxation is usually the fastest process. IC refers to radiationless transitions which occur between isoenergetic vibrational levels of different electronic states of equal spin multiplicity [9]. Therefore, emission of a photon almost always takes place from the lowest vibrational levels of the first excited state, which are populated in thermal equilibrium. Vibrational relaxation from the excited state to the ground state can also occur, e.g. due to collision with solvent molecules [126].

Some electronic transitions are quantum-mechanically forbidden, that is, highly improbable, namely spin-forbidden transitions and symmetry-forbidden transitions. Symmetryforbidden transitions can occur due to molecular vibrations and interactions with

\footnotetext{
${ }^{3}$ A molecule consisting of $\mathrm{N}$ atoms has in total $3 \mathrm{~N}-6$ vibrational modes [116].
} 


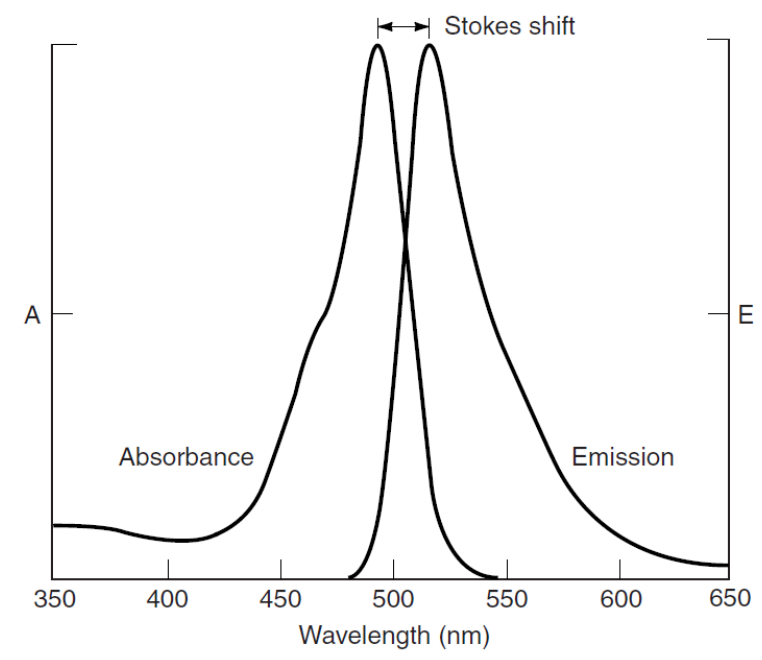

Fig. 2.2: Normalized absorption and fluorescence emission spectra of fluorescein-conjugated immunoglobulin G antibodies. Taken from [88].

solvent molecules. Spin-forbidden transitions, or intersystem crossing between singlet and triplet states, are possible due to spin-orbit coupling, especially when heavy atoms are contained in the molecule. After a transition to the triplet state, the electrons have the possibilities to either transition within the triplet system, or to undergo reverse intersystem crossing back to the excited singlet state, or to decay radiatively to the singlet ground state (phosphorescence) in another spin-forbidden transition.

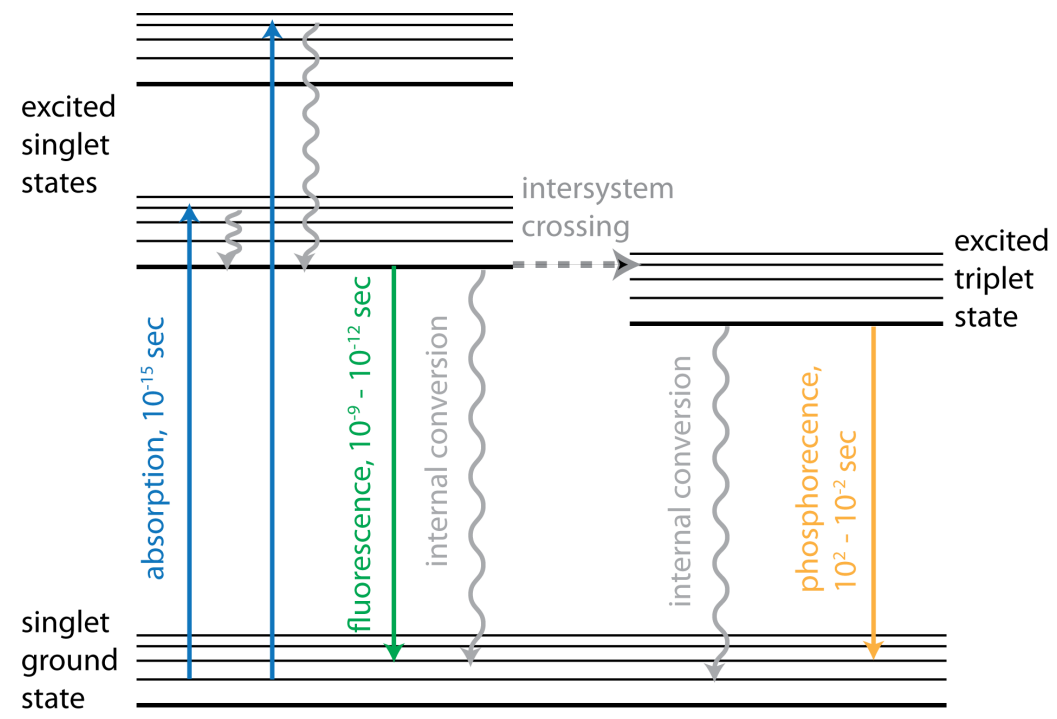

Fig. 2.3: Jablonski diagram depicting a molecule's electronic states and transitions between them. Adapted from [88].

The Jablonski diagram, shown in Fig.2.3, is the established way of visualizing the electronic states of a molecule and all possible radiative and non-radiative transitions between them. With a view to the Jablonski diagram, one observes that fluorescence, internal conversion (IC) and intersystem crossing (ISC) are competing relaxation pathways from the first excited state. The time that the electron spends in the excited 
state, the so-called fluorescence lifetime, is inversely inversely proportional to the rates of the individual transitions:

$$
\tau_{\mathrm{s}}=\frac{1}{k_{\mathrm{r}}^{\mathrm{s}}+k_{\mathrm{ic}}^{\mathrm{s}}+k_{\mathrm{isc}}^{\mathrm{s}}}
$$

where s denotes the singlet state. The same relation holds true for the lifetime of the excited triplet state:

$$
\tau_{\mathrm{t}}=\frac{1}{k_{\mathrm{r}}^{\mathrm{t}}+k_{\mathrm{nr}}^{\mathrm{t}}}
$$

where $\mathrm{r}$ stands for radiative decay and $\mathrm{nr}$ for nonradiative decay.

The quantum yield (QY) of a fluorophore is the ratio of emitted photons to absorbed photons, or, in other words, the probability that an excited electron decays radiatively:

$$
Q Y=\frac{k_{\mathrm{r}}^{\mathrm{s}}}{k_{\mathrm{r}}^{\mathrm{s}}+k_{\mathrm{ic}}^{\mathrm{s}}+k_{\mathrm{isc}}^{\mathrm{s}}}
$$

It is proportional to the fluorescence lifetime $\tau_{\mathrm{s}}$. With the QY, the brightness of a fluorophore upon excitation at a given wavelength can be defined as $\varepsilon_{\mathrm{A}}(\lambda) \cdot Q Y$.

\subsection{Stimulated emission}

As discussed above, a photon can induce an electronic transition (stimulated absorption) if its energy matches the energy difference between the initial and final state, which means that it meets the resonance condition. In addition to stimulated absorption described before, the reverse process of stimulated emission also exists. Both processes are essentially the same and occur with the same probability. If a photon which fulfils the resonance condition hits a molecule in the excited state, then instead of being absorbed, it stimulates the emission of a second photon. The incoming and the stimulated photon are coherent, which means they propagate in the same direction with the same phase and have the same wavelength. This can be derived from theoretical considerations, as explained in detail in [116].

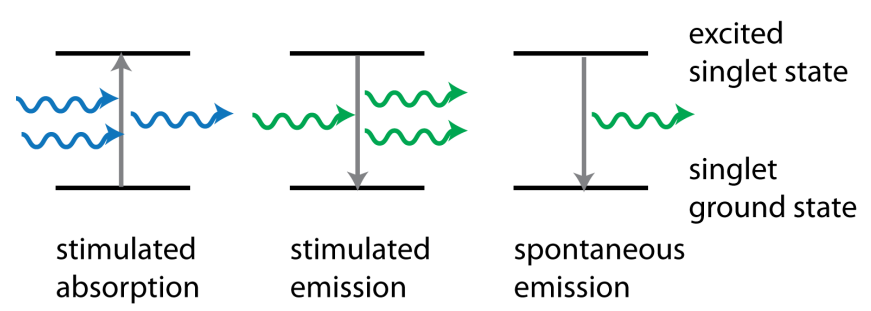

Fig. 2.4: Schematic of stimulated absorption and stimulated and spontaneous emission. Adapted from [86].

In order to understand why mostly stimulated absorption and spontaneous emission of fluorescence, but hardly any stimulated emission are observed, let's consider a model 
system with two energy levels as shown in Fig.2.4. Each energy level $E_{1}$ and $E_{2}$ is populated by a certain number of molecules $N_{1}$ and $N_{2}$. The ratio of $N_{1}$ to $N_{2}$ in thermal equilibrium obeys the Boltzmann distribution:

$$
\frac{N_{1}}{N_{2}}=\exp \left(-\frac{E_{1}-E_{2}}{k T}\right)=\exp \left(\frac{h v}{k T}\right)
$$

It follows that in thermal equilibrium almost all molecules are in the ground state and therefore stimulated absorption is much more likely than stimulated emission.

The state population changes upon interaction with a light field can be quantified using the Einstein probability coefficients for each possible transition: $B_{12}$ is the induced absorption coefficient, $B_{21}$ the stimulated emission coefficient and $A_{21}$ the spontaneous emission coefficient. They are defined by the following rate equation:

$$
\frac{d N_{1}}{d t}=-\frac{d N_{2}}{d t}=-B_{12} \rho(v) N_{1}+B_{21} \rho(v) N_{2}+A_{21} N_{2}
$$

where $\rho(v)$ is the energy density of the radiation field per frequency interval.

By solving the equation for $\rho(v)$ and assuming $N_{1}$ and $N_{2}$ in thermal equilibrium one obtains

$$
\rho(v)=\frac{A_{21}}{B_{12} \exp \left(-\frac{E_{1}-E_{2}}{k T}\right)-B_{21}}
$$

By identifying this with the Planck radiation law, we can deduce

$$
B_{12}=B_{21} \quad \text { and } \quad \frac{A_{21}}{B_{21}}=\frac{16 \pi^{2} \hbar v^{3}}{c^{3}}
$$

thus proving that stimulated absorption and emission are indeed equally probable, as stated above.

In a STED microscope, the excitation pulse first increases $N_{2}$, thereby increasing the rate of stimulated emission when the second laser pulse hits the sample. If there are molecules remaining in the ground state, $N_{1}>0$, absorption and subsequent emission can also take place, as described in chapter 17. 


\section{Photochemistry of organic fluorophores}

In this chapter, various photochemical phenomena observable with organic fluorophores are summarized, which are either essential features or major issues in different nanoscopy techniques. Knowledge of these phenomena is also important for the correct analysis of single molecule FRET data.

\subsection{Fluorescence quenching}

Fluorescence quenching is the loss of fluorescence intensity due to a shortened fluorescence lifetime and/or a lower QY. It can be caused by different intrinsic or intermolecular processes, all of which occur on the same timescale as spontaneous emission [126]:

- intramolecular de-excitation:

- oxygen-catalyzed intersystem crossing [67]

- charge transfer

- internal rotation

- proton transfer

- intermolecular processes:

- collision with heavy atom-containing or paramagnetic molecules [126]

- electron transfer

- proton transfer

- FRET

- excimer ${ }^{1}$ or exciplex ${ }^{2}$ formation (at high concentrations, usually due to $\pi-\pi$ interaction)

- photochemical de-excitation by breaking and formation of new bonds

One can distinguish between static and dynamic quenching, the latter is influenced by the diffusion kinetics of both interacting species (Stern-Volmer kinetics). Both dynamic quenching and transient dark states are fundamental for various single molecule

\footnotetext{
${ }^{1}$ Dimer of two molecules of the same kind, one of which is in the excited state.

${ }^{2}$ Excited state complex of two different excited molecules.
} 
localization microscopy (SMLM) techniques, which are outlined in section 5.2. They can be controlled by the buffer composition.

\subsection{Transient dark states}

The transition of fluorophores to short-lived non-fluorescent (dark) states causes fluorescence intermittencies, which is called blinking. These transient dark states can be of different nature [77]:

- triplet states

- semi-reduced fluorophore radicals

- thiol and phosphine adducts

- leuco forms of photochromic fluorophores (refer to section 3.5)

Triplet states usually have a lifetime of a few $\mu$ s in aqueous buffers, because they are efficiently quenched by molecular oxygen. If oxygen is removed from the solution, the triplet state lifetime increases to ms [45]. Since the triplet state has such a long lifetime, the probability for collision-induced reactions is increased, which may lead to photobleaching (refer to section 3.4).

Fluorophore radicals, which are generally non-fluorescent, are formed by photoinduced electron transfer. This is more likely to occur from the triplet state. By adding reducing and oxidizing agents (ROXS, refer to section 16.1) to the buffer and removing oxygen, radicals and photoionized species can be recovered and triplet states quenched, such that blinking and photobleaching are minimized [130].

For FRET, it is important to note that even though the acceptor in the triplet state does not emit ${ }^{3}$, it can often still absorb energy from the donor. This appears as collective quenching in single pair fluorescence time traces.

\subsection{Spectral diffusion}

The term spectral diffusion describes the change of spectral features of fluorophores, namely their absorption and emission spectra and spectral width, over time [2]. Usually, changes on the timescale of hundreds of $\mathrm{ms}$ to min are reported. Spectral diffusion is often attributed to changes in the local environment of the fluorophore, such as:

\footnotetext{
${ }^{3}$ Fluorescence emission after electronic transitions between the triplet ground state $T_{1}$ and the excited triplet state $T_{2}$ can occur, but usually it is shifted to longer wavelengths.
} 
- strain or local electric fields for single molecules embedded in a crystal [2]

- low-frequency excitation of an amorphous polymer matrix ([65], [115])

- reorientation of matrix molecules changing the local polarizability [122]

- thermal fluctuations [145]

- change of polarity [44]

- conformational changes or perturbations induced by excess energy released in nonradiative relaxation ([145], [36])

- transitions between metastable minima in the molecule's potential-energy surface, either intramolecular conformations or hydrogen bonds to molecules in the environment ([82], [120])

The dependence of a dye's spectral features on environmental parameters actually enables its use as a fluorescence indicator for electrostatic potential, $\mathrm{pH}$, hydrophobicity or the concentration of certain ions, as was noted by [68] and [16].

\subsection{Photobleaching}

Photobleaching is the chemical alteration of a fluorophore, such that it is permanently unable to emit fluorescence. Thus it is a major hindrance in most fluorescence techniques. There are two main mechanisms of photobleaching, namely photo-oxidation and photoionization [135]. Photo-oxidation occurs if singlet oxygen is generated by sensitization of oxygen molecules, which reside in the triplet ground state, by fluorophores in the triplet state. Photoionization can happen after the excitation of fluorophores to first or higher excited singlet or triplet states and is more likely in polar solvents. The arising fluorophore radicals and solvated electrons are highly reactive.

Consequently, photobleaching can be minimized experimentally by:

- decreasing the oxygen concentration

- quenching of the triplet state

- avoiding multiphoton excitation

- in STED, using longer STED laser pulses with lower peak powers [91]

Oracz et al. [91] found that in case of STED there is both intensity-dependent bleaching from higher excited singlet and triplet states and intensity-independent bleaching from the first triplet state. 


\subsection{Photochromism / Photoswitching}

Photochromism, also referred to as photoswitching, is the reversible chemical transformation of a fluorophore between two forms that have different absorption spectra [67]. The transformation in one or both directions can be induced by the absorption of light of the appropriate wavelength. Often, fluorophores change their color in the light and return to their original color in the dark [57]. The underlying mechanism can be cis-trans isomerization or a cyclization reaction. If one form of the fluorophore appears colorless, it is called the leuco form. Photoswitching is essential for the function of SMLM and RESOLFT nanoscopy, as outlined in chapter 5.

\subsection{Photoactivation}

Photoactivatable fluorophores, also called caged fluorophores, are originally nonfluorescent and can be photochemically converted to efficient fluorophores, i.e. activated [74]. During this photoactivation by irradiation with light of the appropriate wavelength, a photolabile chemical group (caging group) is irreversibly removed. This is the basis of PALM microscopy, refer to section 5.2.

\subsection{Photoconversion}

Photoconversion of fluorophores (often uncontrolled) by light induced chemical reactions from higher excited or triplet states has previously been described in literature. The chemical mechanism of the observed photoconversion is not always established. Irreversible red-to-blue photoconversion of Cy3.5 and Cy5 upon two-photon excitation has been reported by Kwok et al. [70], who conducted cell staining experiments. Liao et al. found green emitting photoproducts from terrylene diimide [76] in both dye solution and single molecule measurements. Additionally, photoconversion of tris(diethylamino)-trioxatriangulenium, which is similar to rhodamine, to both blueand red-shifted photoproducts has been observed in ensemble measurements by Liao et al. upon excitation with blue light [75]. They state that this "might help to explain spectral shifts observed for rhodamine B and Texas red" ([60], [132]), which are commonly used dyes. Photoconversion of fluorophores Atto532 and Star635P to various red- and blue-shifted products is described in chapter 21 . 


\section{Theory of light microscopy}

\subsection{Image formation in the light microscope}

A microscope is an optical instrument which allows the user to view objects that are too small to be seen by the naked eye. Magnification of the object is achieved in two stages by two essential components: The objective forms an enlarged, real image of the object inside the microscope. The ocular, also called eyepiece, projects a magnified, virtual, inverted image of that real image to the eye. This is illustrated in Fig.4.1. Often, infinity-corrected microscopes, which feature a tube lens between objective and ocular as depicted in Fig.4.2, are used. Between objective and tube lens, the rays run parallel, therefore the optical path length is not critical. This permits the insertion of additional planar optical components, such as beam splitters or filters.

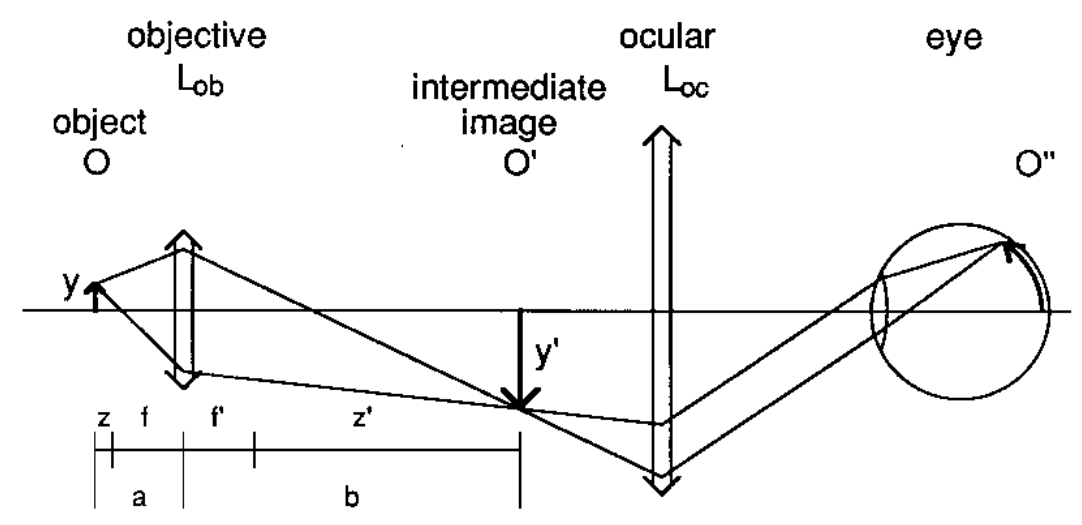

Fig. 4.1: Ray path in the microscope from the object to the observer's eye. Taken from [46].

There are two ways in which the object can be illuminated: In transillumination mode, light is shone onto the sample through a condenser lens, which opposes the objective lens. In epi-illumination mode, the sample is illuminated through the objective lens. In the work presented here, epi-illumination through an infinity-corrected objective was used. The final image can either be viewed through the ocular by eye or the intermediate image O' can be detected by a camera sensor. This is called wide-field microscopy, in contrast to confocal microscopy, which will be explained in section 4.4.

The total magnification $M_{\mathrm{tot}}$ of the microscope is

$$
M_{\mathrm{tot}}=M_{\mathrm{ob}} \cdot M_{\mathrm{oc}}
$$




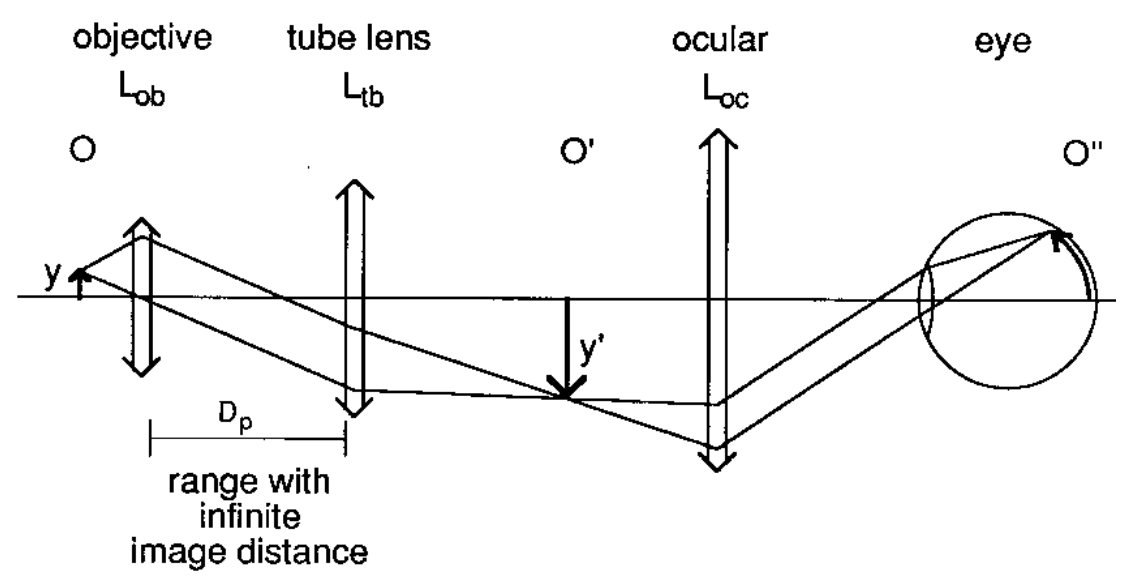

Fig. 4.2: Ray path in a microscope with infinity-corrected objective and tube lens. Taken from [46].

with $M_{\mathrm{oc}}=25 \mathrm{~cm} / f_{\mathrm{oc}}$ and $M_{\mathrm{ob}}=b / a=f / z=y^{\prime} / y$. The standardized distance of $25 \mathrm{~cm}$ is the so-called near point, the shortest reading distance most people can accommodate without visual aids.

The microscope's resolving power as well as its light gathering power are determined by the objective's numerical aperture (NA). It is defined as

$$
\mathrm{NA}=n \cdot \sin (\theta)=n \cdot \frac{d}{2 f}
$$

where $n$ is the index of refraction, $\theta$ is the one-half angular aperture of the objective's illumination and detection light cone, $d$ is the diameter of the objective's aperture and $f$ its focal length.

The image formation in a microscope can be described with the help of convolution and the Fourier transformation. The intermediate image of an object point-like source is not reproduced as a point, but as a three-dimensional diffraction pattern called point spread function (PSF). This is due to diffraction at the objective aperture that acts as a low-pass filter for spatial frequencies of the object distribution. Wavefront distortions, i.e. abberations, introduced by the optical system further widen the diffraction pattern. The thus-determined PSF blurs the geometric image of the object [78]. The image formed by the ocular is the Fourier transform of the intermediate image. During image formation, the intensity of each spatial frequency contained in the object is multiplied with the optical transfer function ${ }^{1}$ of the respective lens. ${ }^{2}$ Thus, the microscope filters spatial frequencies linearly. Those above a certain cut-off frequency, where the optical transfer function falls off to zero, are not transmitted. Thus, the resulting image is a convolution of the object intensity distribution and the PSF of the microscope.

\footnotetext{
${ }^{1}$ Fourier transform of the PSF.

${ }^{2}$ Note that the convolution of two functions is equal to the multiplication of the Fourier transforms of both functions.
} 


\subsection{Resolution and the diffraction limit}

Due to the objective's finite aperture, the excitation light forms a three-dimensional diffraction pattern at the focus and conversely the emitted light at the detector. In the case of far-field diffraction at a circular aperture, this is the Airy pattern. Its size is set by the NA of the objective and the wavelength $\lambda$ of the light. The axial extent, which corresponds to the depth of field, is

$$
z \simeq \frac{2 \lambda \cdot n}{(\mathrm{NA})^{2}}
$$

The lateral extent of the central Airy disk is

$$
d_{\mathrm{Airy}} \simeq \frac{1.22 \lambda}{\mathrm{NA}}
$$

In the field of microscopy, the term resolution is not defined uniquely. Classically, the resolving power of a microscope is determined by its ability to distinguish two point sources which are a certain distance apart. There are two established criteria for distinguishability: The Rayleigh criterion states that the two point sources can be resolved when the maximum of the first Airy disc overlaps with the first minimum of the second Airy disk, then $d_{\text {Rayleigh }} \simeq 0.61 \lambda /$ NA. The Sparrow criterion defines the smallest resolvable distance between equally bright point sources as the one at which the minimum between the two maxima in the summed intensity distribution of both Airy disks vanishes, $d_{\text {Sparrow }} \simeq 0.47 \lambda / \mathrm{NA}$. Both criteria assume noise-free images.

Resolution can be defined more clearly in the spatial frequency domain. Due to the finite size of the objective aperture, spatial frequencies above a certain cut-off frequency are not transmitted. This cut-off frequency represents the diffraction limit. As a matter of fact, the product of the minimal distance according to the Rayleigh criterion and the cut-off frequency is constant, which shows that both definitions are related [108].

In images obtained with a fluorescence microscope, the full width at half maximum (FWHM) of a single emitter, which can be regarded as a point source, can be measured easily. The FWHM can then serve as an approximation of the resolution according to the Sparrow criterion. Alternatively, to ascertain the spatial resolution of a given microscope image, one can apply a method based on Fourier ring correlation to determine the effective cut-off frequency, the inverse of which is the effective resolution [121].

The term superresolution was originally coined in the field of electron microscopy for methods which aim to reconstruct spatial frequency components beyond the cut-off frequency of the microscope. To achieve this, prior knowledge is fed into a model, 
a
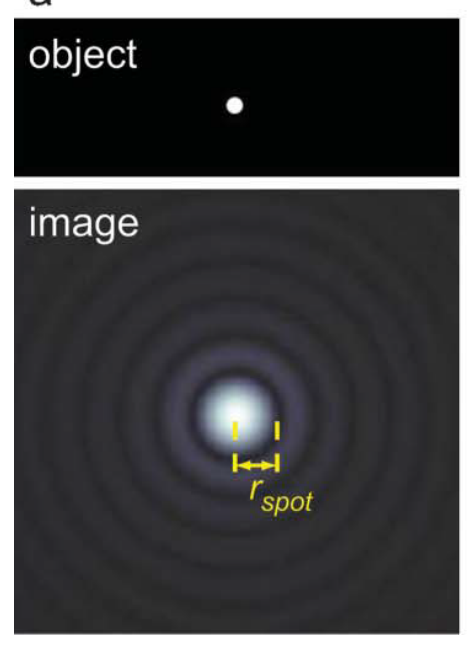

b
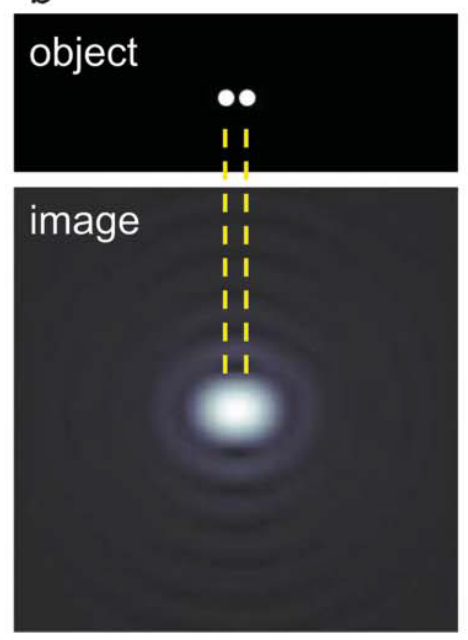

C
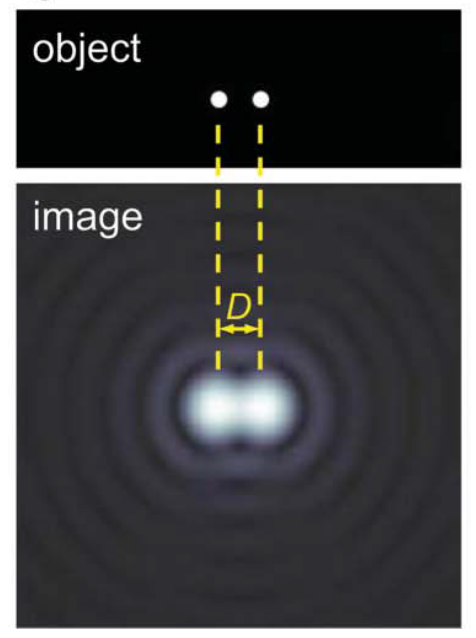

Fig. 4.3: Conceptual-model illustration of the Rayleigh criterion for the minimum resolvable distance D between two small point-like objects. (a) The diffraction-limited predicted image (lower panel) of a small object (upper panel) consisting of a bright sphere. (b) The corresponding image of two such small objects, unresolved case. (c) Same as in (b) but the limit where the spacing, D, is just large enough for resolving the two small spheres. The radius of the imaged spot, $r_{\text {spot }}$, in (a) is equal to $\mathrm{D}$ in (c). The situation in (c) illustrates the Rayleigh criterion. Adapted from [136].

turning an image reconstruction problem into a parameter estimation problem. A good introduction can be found in chapter 20 of [108]. However, the term is often misunderstood in the field of fluorescence microscopy with techniques such as STED, where no model-based image reconstruction is necessary to overcome the diffraction limit.

\subsection{The fluorescence microscope}

One way to generate contrast in microscope images is to use fluorescent molecules as markers for structures of interest. Due to the Stokes shift, the excitation light and the emitted fluorescence can be separated with dichroic filters as depicted in Fig.4.4, such that only the fluorescence light is detected. Thus, one obtains images where only the structure of interest is visible above a dark background.

Fluorescence microscopy offers several unique advantages: Living biological samples can be imaged noninvasively in three dimensions. Structures of interest can be labeled with high specificity and observed with great contrast. Endogenous fluorescence markers can be used, reducing the complexity of sample preparation and thereby the risk of artefacts. Fluorophores which are sensitive to certain environmental parameters can be used to probe properties such as $\mathrm{pH}$, metal ion concentration, oxygen concentration, viscosity, solvent polarity, membrane potential, temperature or pressure locally [126]. 


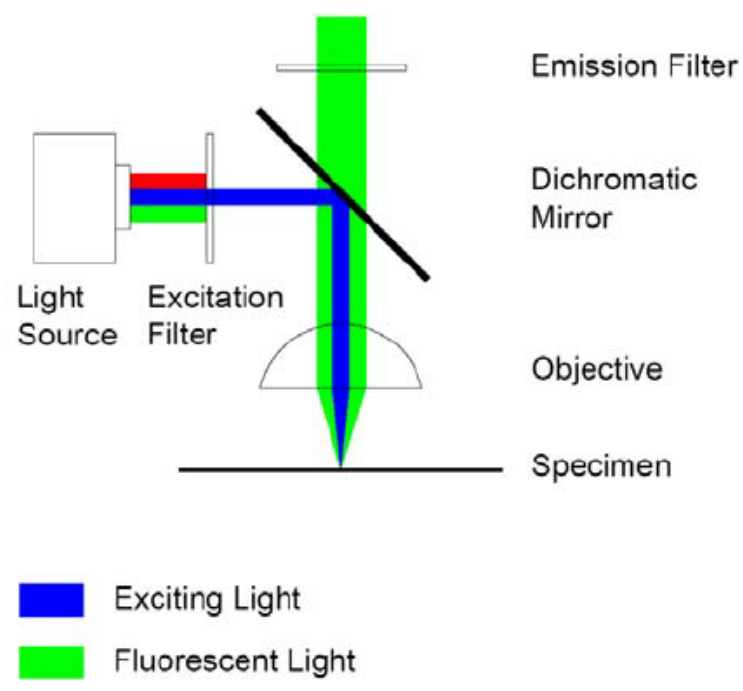

Fig. 4.4: Working principle of the fluorescence microscope, taken from [103].

\subsection{The confocal microscope}

Although fluorescence microscopy has a good contrast, it still suffers from background light generated outside the depth of field. This out-of-focus light can be blocked by a pinhole placed in a conjugate plane to the focal plane (thus "confocal") in front of a point detector, as sketched in Fig.4.5. In order to acquire an image, the excitation laser focus is scanned across the sample and at each point the fluorescence is collected by the detector. In this manner, the signal-to-background ratio is increased. The resulting image is a convolution of the object intensity distribution with the convolution of the PSF of the objective and the pinhole aperture function.

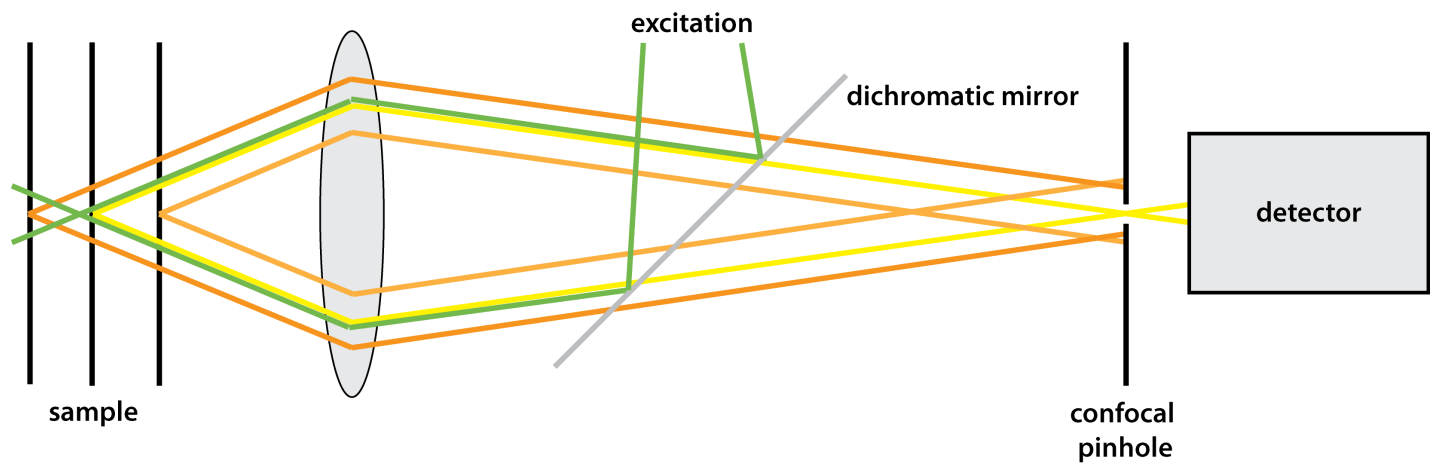

Fig. 4.5: The confocal pinhole rejects light from out-of-focus planes. 



\section{Nanoscopy}

\subsection{Coordinate-targeted nanoscopy}

Deterministic nanoscopy methods achieve diffraction-unlimited resolution by driving reversible saturable/switchable optical linear (fluorescence) transitions (RESOLFT) in a coordinate-targeted manner with illumination patterns, usually a torus-shaped focus or a grid [33]. The RESOLFT concept has been realized experimentally using different transitions of the fluorophores: stimulated emission depletion (STED) microscopy [43], as the name suggests, uses stimulated emission to deplete the fluorescence in the outer rim of an excitation spot. Ground state depletion (GSD) microscopy [18] achieves diffraction-unlimited resolution by transferring part of the molecules to a metastable dark state, such as the triplet state, before image acquisition. RESOLFT [42] was also performed by shifting reversibly switchable fluorescent proteins into their bright and dark states prior to imaging. Multiple transitions can be combined to achieve higher contrast and resolution at smaller light doses and less photodamage, as implemented in protected STED (MOST) [29].

\subsubsection{Stimulated emission depletion nanoscopy}

The point-scanning STED microscope enhances the confocal fluorescence microscope to achieve diffraction-unlimited resolution. It overcomes the diffraction barrier by toggling fluorophores between the non-fluorescent ground state and the first exited state in a targeted manner with STED.

Two diffraction-limited laser foci are employed in a STED microscope, as illustrated in Fig. 5.1: The Gaussian focus excites the fluorophores. Immediately afterwards, a torus-shaped ("doughnut-shaped") focus transfers the fluorophores in the periphery of the excitation spot back to the non-fluorescent ground state by STED. The fluorophores in the center of the STED doughnut-focus receive no STED light and are allowed to emit fluorescence spontaneously. Thus, the STED microscope sets up an effective PSF by confining the region in which fluorophores remain excited. Both laser beams are superimposed and scanned across the sample simultaneously to read out fluorescence, as depicted in Fig. 5.2. As the effective PSF is smaller than the diffraction limit, 


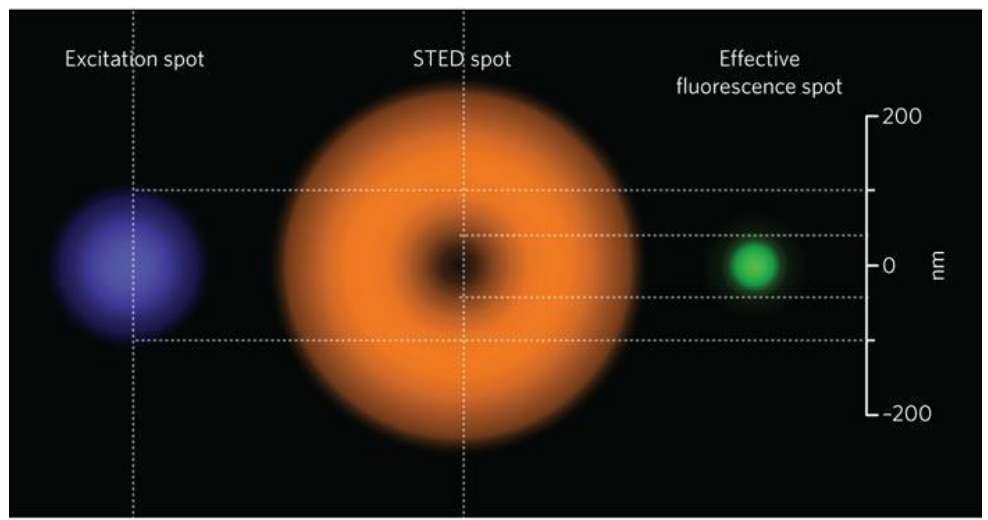

Fig. 5.1: STED mode of operation: The diffraction-limited excitation focus excites the fluorophores. The torus-shaped STED focus transfers the fluorophores in the periphery of the excitation spot back to the non-fluorescent ground state. The remaining fluorophores in the center of the STED doughnut-focus are allowed to emit fluorescence. Taken from [1].

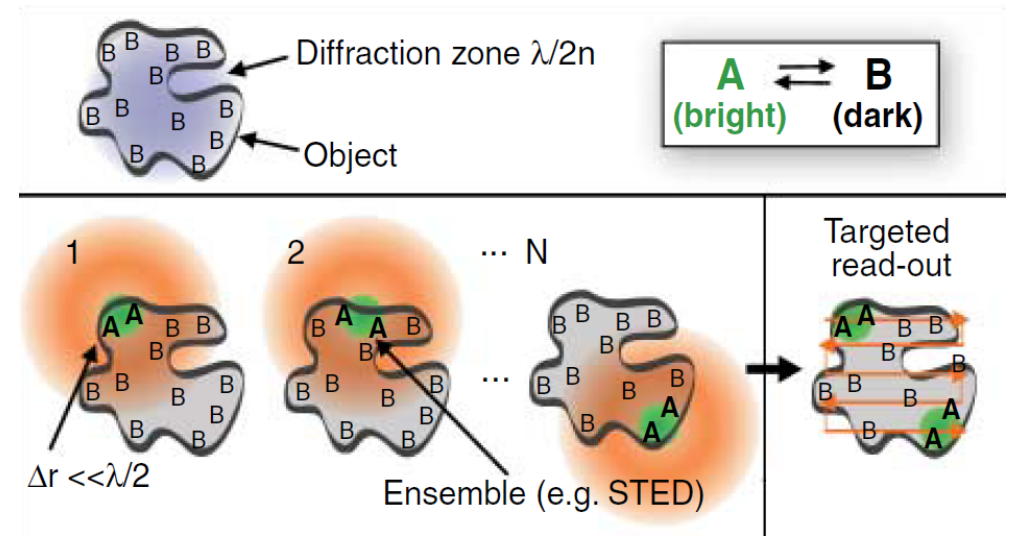

Fig. 5.2: Coordinate-targeted read-out with STED: Excitation focus (blue) and STED focus (orange) are superimposed and scanned across the sample simultaneously. Only fluorophores in the effective excitation spot (green) emit fluorescence, as illustrated in Fig. 5.1. Thus fluorophores spaced closer than the diffraction limit are read out sequentially to obtain a diffraction-unlimited image. Adapted from [49].

fluorophores spaced close together are detected sequentially, thus the diffraction limit is overcome.

The torus-shape of the STED focus can be generated by passing a circularly polarized laser beam through a vortex phase plate, which induces a helical phase shift from zero to $2 \pi$. The phase shift causes destructive interference of all field components when the laser beam is focused, as illustrated in Fig. 5.3.

The diameter of the effective excitation spot is dictated by the STED laser intensity. The higher the maximum STED intensity $I_{\max }$, the closer to the zero it exceeds the 


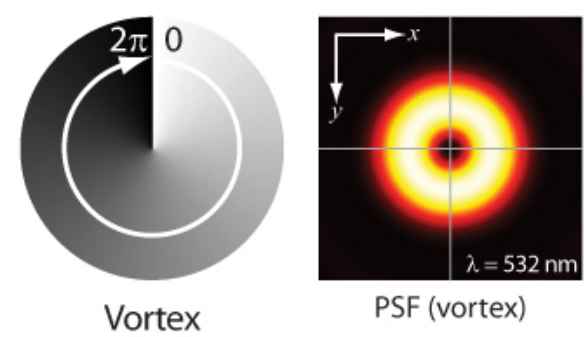

Fig. 5.3: A vortex phase plate (left) induces a helical phase shift in the circularly polarized STED beam, which creates a doughnut-shaped focus (right) by destructive interference. Taken from [137].

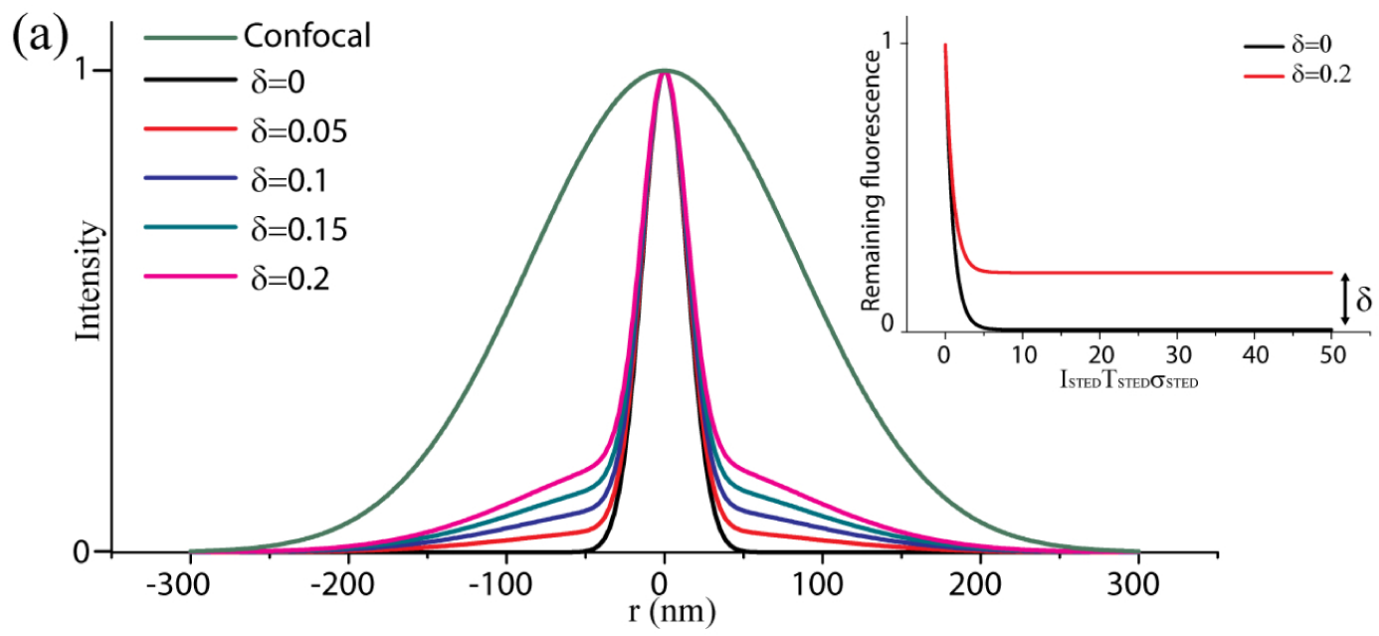

Fig. 5.4: Simulated effective STED PSF for different incomplete inhibition factor $\delta$. Incomplete depletion was modeled by adding the constant offset $\delta$ in the spontaneous emission probability. Adapted from [38].

saturation intensity $I_{S}$ of the depletion transition. Thus the lateral spatial resolution of the STED microscope is defined as follows [49]:

$$
d=\frac{\lambda}{2 n \cdot \sin (\alpha) \cdot \sqrt{1+\frac{I_{\max }}{I_{\mathrm{S}}}}}
$$

The axial resolution is not altered by the torus-shaped STED focus.

Additionally, the lateral resolution of the STED microscope is influenced by the temporal delay with which excitation and STED pulse hit the sample. If the STED pulse comes too early, the fluorophores are not excited yet or have not yet relaxed to the lowest vibrational level of the first excited state, thus impeding STED. If the STED pulse reaches the sample too late, fluorophores will have emitted fluorescence spontaneously in the mean time, causing a pedestal in the PSF. This is shown in Fig. 5.4. The pedestal due to early emission can be removed by time gating at the cost of decreasing the overall signal. 


\subsection{Single molecule localization microscopy}

As the name implies, single molecule localization microscopy (SMLM) is achieved through the sequential localization of single, temporally and spatially non-overlapping emitter images in a series of time frames to reconstruct a final, diffraction-unlimited image. The localization precision, and thus the achievable resolution, scales with the square root of the number of photons collected. In order to achieve a sufficient signalto-noise ratio (SNR), the microscope is often used in a total internal reflection (TIRF) illumination mode [35]. A diversity of molecular states and transitions can be used to achieve the activation of only a sparse subset of molecules in each time step. There are irreversible transitions, such as photoactivation, photoconversion and bleaching, which are used in photoactivated localization microscopy (PALM). Furthermore there are reversible transitions, such as photoswitching and blinking, used in STORM, and binding/unbinding, which are used in point accumulation for imaging in nanoscale topography (PAINT) [109]. All these transitions can be found in both organic dyes

and fluorescent proteins and all, except transient binding, can be driven by light of the appropriate wavelength. Using several cameras for detection in separate spectral bands is possible. Further information can be found in [50], [33] and the references therein.

\subsection{Nanoscopy with minimal emission fluxes}

MINFLUX [10] combines the stochastic photoswitching necessary for SMLM with a doughnut-shaped excitation focus, reminiscent of RESOLFT approaches, for locating a fluorophore [114]. MINFLUX sequentially pinpoints fluorescence emitters using the local intensity minimum of the excitation focus, thus minimizing the number of photons needed for precise localization. This method can be used for imaging as well as for single molecule tracking. A localization precision of about $1 \mathrm{~nm}$ has been demonstrated experimentally using 500 photons, which would require about 20,000 photons or more in SMLM. Thus, two molecules which were spaced $6 \mathrm{~nm}$ apart were resolved clearly. Thereby, MINFLUX is a nanoscopy method which already at its first demonstration entered the domain of inter-dye distances that were so far measurable by FRET only. 


\section{Förster resonance energy transfer}

The first section of this chapter presents the physical theory of Förster resonance energy transfer (FRET). In the second section, different methods to quantify the FRET efficiency from experimental data are introduced. Biological applications of FRET are outlined in section 7.2. Further information about FRET can be found in [84], [71], [126] and [94], amongst others.

\subsection{Theory of FRET}

The term Förster resonance energy transfer (FRET) describes the non-radiative ${ }^{1}$ transfer $^{-}$ of energy from an excited donor molecule to a nearby ground-state acceptor molecule, which is converted to the excited state and subsequently emits fluorescence. The transfer is mediated by dipole-dipole interaction between the transition dipole moments of both fluorophores. The transition dipole moment is the electric dipole moment connected with the transition between two electronic states:

$$
M_{n, 0 \rightarrow m, v}=e\left\langle\Psi_{n, 0}|\hat{M}| \Psi_{m, v}\right\rangle
$$

It is not the same as the permanent dipole moment of a molecule. As it results from a dipole-dipole interaction, the FRET efficiency $E$ depends on the inter-dye distance as $r^{-6}$ :

$$
E=\frac{k_{\mathrm{T}}}{1 / \tau_{\mathrm{D}}+k_{\mathrm{T}}}=\frac{R_{0}^{6}}{r^{6}+R_{0}^{6}}
$$

with the donor lifetime $\tau_{\mathrm{D}}$ and the Förster radius $R_{0}$, which is defined below. The energy transfer rate $k_{\mathrm{T}}$ is defined as:

$$
k_{\mathrm{T}}=\frac{1}{\tau_{\mathrm{D}}} \cdot \frac{R_{0}^{6}}{r^{6}}
$$

Since the Förster theory is based on the ideal dipole approximation, it is only valid in the near-field, for distances of approximately $1 \mathrm{~nm}$ to $10 \mathrm{~nm}$. Intermolecular orbital overlap and multipolar interactions are thus excluded [126]. The dynamic range of $E$ is between $0.5 R_{0}$ and $2 R_{0}$ [110], which usually corresponds to inter-dye distances of $2 \mathrm{~nm}$ to $8 \mathrm{~nm}[94]$.

\footnotetext{
${ }^{1}$ Non-radiative means that no photon is emitted and then re-absorbed [71].
} 
Secondly, the efficiency of FRET depends on the relative orientation of the two transition dipole moments, which is described by the orientation factor $\kappa$ :

$$
\kappa=\vec{n}_{\mathrm{D}} \cdot \vec{n}_{\mathrm{A}}-3\left(\vec{n}_{\mathrm{A}} \cdot \vec{r}\right)\left(\vec{n}_{\mathrm{D}} \cdot \vec{r}\right)
$$

where $\vec{r}$ denotes the unit vector joining donor and acceptor and $\vec{n}_{\mathrm{D}}$ and $\vec{n}_{\mathrm{A}}$ are the unit vectors of the transition dipole moments [126]. Usually, freely rotating fluorophores are assumed, such that $\kappa^{2}=2 / 3$. Whether this assumption is valid can be tested with anisotropy measurements.

The third precondition for FRET to occur is that the resonance frequencies of both dipoles have to match, which means that the emission spectrum of the donor and the absorption spectrum of the acceptor have to overlap. This is specified by the spectral overlap integral $J$ :

$$
J(\lambda)=\int_{0}^{\infty} \epsilon_{\mathrm{A}}(\lambda) \cdot \lambda^{4} \cdot F_{\mathrm{D}}(\lambda) d \lambda
$$

where $\epsilon_{\mathrm{A}}(\lambda)$ is the molar absorption coefficient and $F_{\mathrm{D}}(\lambda)$ is the donor emission spectrum normalized to an area of one. FRET between different fluorophores is called heteroFRET. Transfer can also occur between fluorophores of the same kind if their Stokes shift is small, such that their absorption and emission spectra overlap. Then it is termed homo-FRET.

The Förster radius $R_{0}$ subsumes the contributions of orientation factor $\kappa$, overlap integral $J(\lambda)$, the donor quantum yield $Q Y_{D}$ and the refractive index $n$ of the medium [84]:

$$
R_{0}=\left(\frac{9(\ln 10)}{128 \pi^{5} N_{\mathrm{A}}} \cdot \frac{\kappa^{2} \cdot Q Y_{\mathrm{D}} \cdot J(\lambda)}{n^{4}}\right)^{1 / 6}=0.021\left(\frac{\kappa^{2} \cdot Q Y_{\mathrm{D}} \cdot J(\lambda)}{n^{4}}\right)^{1 / 6}
$$

$R_{0}$ is equal to the distance at which the FRET efficiency $E$ is $50 \%$. It is used to characterize FRET pairs.

Due to its strong distance dependence and sensitivity to fluorophore orientation, FRET became a favorite tool in the life sciences, which can determine either the distance of labeled species or their concentration/stoichiometry in close proximity. For example, FRET can measure the conformational changes of proteins, detect protein-protein interactions and oligomerization or function in fluorescent biosensors for small molecules and ions. Several applications are outlined in section 7.2. 


\subsection{Quantification of FRET}

The occurrence of FRET manifests itself in several ways:

- The fluorescence intensity of the donor decreases. The acceptor does not have to fluoresce, it only needs to accept energy from the donor and can also decay non-radiatively. But if it emits, the fluorescence emission of the acceptor increases simultaneously.

- The fluorescence lifetime of the donor is shortened, whereas the fluorescence lifetime of the acceptor grows.

- The fluorescence anisotropy upon linearly polarized excitation is reduced.

All of these effects can be used to experimentally ascertain FRET and to quantify the FRET efficiency, as will be described below. Alternatively, the relative concentration of interaction partners and stoichiometry of complexes can be determined.

\subsubsection{Fluorescence intensity-based methods}

Intensity-based FRET quantification methods are most commonly used, as they require only basic microscope equipment and are easy to perform [54]. Different intensity-based approaches are compared in review [147]. In experiments, the excitation intensity has to be monitored and for analysis, the QY of donor and acceptor as well as the detection efficiency have to be known. Control measurements with only donor and acceptor are needed to correct for spectral crosstalk.

\section{Ratiometric imaging}

The fluorescence emitted upon donor excitation is collected in two spectral channels, which are usually defined by filters. One channel is centered on the donor $\left(I^{D}\right)$ and the other on the acceptor emission band ( $I_{\text {FRET }}^{A}$ ), and the intensity ratio is subsequently analyzed. If the FRET efficiency is high, the donor fluorescence emission will be very low, because almost all the energy is transferred to the acceptor, which will consequently show very bright emission, and vice versa. With ratiometric imaging, it is possible to determine the exact transfer efficiency as:

$$
E_{\mathrm{FRET}}=\frac{I_{\mathrm{FRET}}^{A}}{I^{D}+I_{\mathrm{FRET}}^{A}}
$$

For this, care has to be taken to correct $I_{\mathrm{FRET}}^{A}$ for donor emission bleedthrough into the acceptor channel and for direct cross-excitation of the acceptor, and to subtract back- 
ground signal. The corresponding image acquisition and analysis scheme is illustrated in supplementary Fig. 28.4. The correction factors for donor bleedthrough and acceptor cross-excitation are determined from measurements of samples containing only donor or acceptor.

\section{Spectral unmixing}

A spectral detector can be used to acquire the entire emission spectrum. This can then be analyzed with a spectral unmixing algorithm to determine the contributions of paired and unpaired donors and acceptors, which are measured beforehand in singly labeled samples. Since highly overlapping spectra can be distinguished, highly efficient FRET pairs with large $J(\lambda)$ can be observed. Furthermore, several spectrally distinct FRET pairs can be used simultaneously.

\section{Donor photobleaching}

When FRET occurs, the donor spends less time in the excited state. Therefore, it is less prone to photobleaching. Thus, the energy transfer can be inferred from the bleaching time constant of the donor [110]. However, one has to account for other environmental factors such as the oxygen concentration that also influence the bleaching behavior.

\section{Acceptor photobleaching}

If the acceptor photobleaches, FRET is abolished and the donor is dequenched. By comparing the donor fluorescence intensity before and after acceptor bleaching, one can calculate the FRET efficiency. Unfortunately, information is lost if the donor bleaches first. Further, it is difficult to monitor the generation of dark acceptors which still absorb, as well as the generation of photoconverted fluorophores. 


\subsubsection{Fluorescence lifetime-based methods}

The reduction of the donor's fluorescence lifetime can be used as a measure for the FRET efficiency. The energy transfer constitutes an additional relaxation pathway for the excited donor fluorophore. Thus, the higher the FRET efficiency, the shorter the donor lifetime becomes. By measuring the donor lifetime, the most precise values for the transfer efficiency can be obtained as:

$$
E_{\mathrm{FRET}}=1-\frac{\tau_{\mathrm{DA}}}{\tau_{\mathrm{D}}}
$$

where $\tau_{\mathrm{D}}$ is the unquenched donor lifetime and $\tau_{\mathrm{DA}}$ is the donor lifetime in the presence of the acceptor. Furthermore, it is possible to determine the ratio of FRET active and inactive donors from a biexponential decay fit. The fluorescence lifetime is independent of the excitation intensity and in most cases independent of the local concentration. It is however prone to changes in chemical parameters such as $\mathrm{pH}$, polarity and temperature.

\section{Time-domain measurements}

The fluorescence lifetime can be measured in the time domain using single photon detectors and time correlated single photon counting (TCSPC) electronics. TCSPC can be performed with point detectors such as APDs in laser scanning microscopes. Single photon cameras with a time resolution of ps necessary for measuring ns decay times are currently under development [52].

The arrival times of the fluorescence photons with respect to the excitation laser pulses are registered by the TCSPC electronics and are collected in a lifetime histogram in each image pixel. The histogram is then usually fitted with single or multiple exponential decay functions to extract the fluorescence lifetime. Especially when measuring short decays, the instrument response function (IRF) needs to be characterized either with a scattering sample or with a sample with an extremely short lifetime [117]. Then the lifetime histogram is deconvolved with the IRF before analysis. Further detailed information on TCSPC can be found in [131] and [92].

\section{Frequency-domain measurements}

The fluorescence lifetime can alternatively be measured in the frequency domain using sinusoidally modulated excitation intensity. The fluorescence emission intensity is 
consequently also modulated and the phase shift $\phi$ between excitation and fluorescence emission is related to the fluorescence lifetimes as follows [84]:

$$
\phi=\arctan \left(\frac{\sum_{i=1}^{n} \frac{a_{\mathrm{i}} \omega \tau_{\mathrm{i}}^{2}}{1+\omega^{2} \tau_{\mathrm{i}}^{2}}}{\sum_{i=1}^{n} \frac{a_{\mathrm{i}} \tau_{\mathrm{i}}}{1+\omega^{2} \tau_{\mathrm{i}}^{2}}}\right)
$$

with modulation frequency $\omega$ and lifetimes $\tau_{\mathrm{i}}$. Such experiments can be performed with a camera in a widefield fluorescence microscope. Unfortunately, frequency-domain measurements are less sensitive and accurate than TCSPC measurements at low photon counts. Further information can be found in [37].

\subsubsection{Fluorescence anisotropy-based methods}

The change of fluorescence anisotropy due to FRET after excitation with linearly polarized light can also be used as an observable. There are two opposing trends: On the one hand, since the donor lifetime is shortened, the fluorophore has less time for rotational diffusion, thus the donor emission is more polarized. This can be used to calculate the FRET efficiency as [26]:

$$
E=\frac{P_{0}\left(P_{\mathrm{D}}^{\mathrm{A}}-P_{\mathrm{D}}\right)}{P_{\mathrm{D}}^{\mathrm{A}}\left(P_{0}-P_{\mathrm{D}}\right)}
$$

with the minimal fluorescence polarization $P_{0}$ of the donor, and the polarization of the donor in the presence $P_{\mathrm{D}}^{\mathrm{A}}$ and absence $P_{\mathrm{D}}$ of the acceptor. On the other hand, FRET between a donor and an acceptor that are not parallel leads to depolarization of the acceptor fluorescence emission. This has been used to qualitatively observe FRET. Fluorescence anisotropy-based methods are the only ones that can detect homo-FRET [20]. 
Biological applications of FRET and STED are briefly reviewed in the first chapter of this part to motivate where a combination of both techniques would be beneficial. The advantages that may be gained are highlighted in the second chapter. Even though it would be favorable, FRET and nanoscopy have not been combined so far except in one special case, which is introduced in section 9.4. Thus, a short account is provided in the third chapter of publications in which FRET has been used either to facilitate superresolution imaging or where it has complemented superresolution studies. Hurdles that would have to be overcome to unite FRET with superresolution techniques other than STED are discussed in chapter 25. 



\section{Brief literature review: Biological applications}

\subsection{Biological applications of STED nanoscopy}

STED nanoscopy has been established as a valuable tool in various fields of enquiry in biology because it allows the study of small objects with sizes below the diffraction limit. Several examples are reviewed in [101], such as:

- discoveries in neurobiology

- periodic ring-like structure of actin and associated molecules in neurons and glial cells

- spine-neck morphology and dynamics before and after long-term potentiation

- molecular components co-aligned in pre- and postsynapse

- protein aggregate nucleation and fibril formation in neurodegenerative disorders

- discoveries in mitochondrial biology

- formation of apoptotic pores in the outer mitochondrial membrane

- clustering of nucleoids

- discoveries in virology

- proteolytic maturation of HIV

- ESCRT protein machinery organization at HIV assembly sites at the plasma membrane

- Env clusters at HIV assembly sites [102]

- Herpes virus clustering at the outer mitochondrial membrane [14]

Many reports to date have used STED to study thin, flat cells grown on coverslips, which were often chemically fixed. Mostly protein assemblies with a defined structure, such as the cytoskeleton or nuclear pores, or small volumes, such as synapses, were imaged. In these cases, the advantage of improved resolution was easy to demonstrate.

In the last years, advances in STED microscopes and labeling techniques enabled multicolor live cell imaging ([141], [111], [48]), imaging in tissue slices ([125], [64], 
[59], [124]) and even in live animals ([13], [138], [133]). For the latter, inherent optical sectioning and background suppression due to the confocality of most STED microscopes offer a big advantage.

Time lapse imaging is often required to not just map the distribution of proteins or the static structure of protein complexes, but to elucidate their function in concert with other cellular components. Additionally, to gather enough signal while scanning regions of interest quickly with high resolution, bright and photostable labels are necessary. One prime example of the determination of functional properties has been reported by Richardson et al. [99], who developed ratiometric $\mathrm{pH}$ sensors for the study endosomal pathways with live cell STED.

\subsection{Biological applications of FRET imaging}

The physical phenomenon of FRET occurs between fluorescent dyes which are closer than $10 \mathrm{~nm}$ together and is very sensitive to small distance changes. It is used in conjunction with several fluorescence techniques such as fluorescence microscopy, in vivo imaging of whole animals [22], flow cytometry, immunoassays, real-time PCR assays, automated DNA sequencing, and membrane fusion assays. In this thesis, I focus on applications of FRET in fluorescence microscopy.

Due to its sensitivity to small distance changes, FRET is a suitable tool to monitor protein folding ([107], [5]), conformational changes [83], filament assembly [61] or transient protein interactions [62]. Such measurements are typically performed on ensembles of purified molecules immobilized individually on coverslip surfaces [100]. Since the molecule surface density is freely adjustable, these measurements would not benefit from nanoscopy. Therefore, these applications are not considered in this thesis.

Instead, I focus on imaging studies which are performed in live cells, and which elucidate functions of various proteins and other small molecules by:

- visualizing protein-protein interaction [32]

- measuring oligomerization [11], [98]

- monitoring binding of cofactors and ligands [140], [87]

- measuring enzyme activity [54], [51]

- tracking protein activation by phosphorylation [19], [143]

- reading out FRET biosensors 
FRET-based biosensors constitute a rapidly growing field of research. They are engineered biomolecules containing a FRET pair which change between a high- and low-FRET state in response to an external biological stimulus. Several mechanisms are possible [54]:

- conformational changes between the FRET partners due to ligand binding or changes in the microenvironment

- mechanical forces changing the distance between the fluorophores

- cleavage of the sensor separating the FRET partners

In [54] and [84], biosensors for studying biological processes such as apoptosis, autophagy, signal transduction, and mechanotransduction are listed, in addition to biosensors for measuring metabolite quantification, intracellular $\mathrm{pH}$, oxygen and reactive oxygen species concentration. Zamaleeva et al. [146] report on quantum dot-based biosensors for $\mathrm{Ca}^{2+}$ and $\mathrm{H}^{+}$ions. Furthermore, in [85], numerous biosensors that visualize the spatial activity gradients of kinases, GTPases and G proteins are described. Welch et al. [134] report the use of three biosensors to study the coordination of multiple signalling pathways. Even biosensors for mechanical tension [27] and molecular crowding [28] have been developed. 



\section{Motivation: Combining FRET and STED for functional imaging}

In [40], H. E. Grecco and P. J. Verveer discuss the role of FRET in cell biology in light of the ever increasing spatial resolution of nanoscopy methods. These might indeed become an alternative to FRET measurements in cases where FRET is used as a reporter of colocalization. It should also be possible to replace some cleavage-based FRET biosensors with nanoscopy. A principal advantage of nanoscopy would be that unlike FRET, it could easily measure distances up to tens of nanometers. For example, it could monitor components of protein complexes that are larger in diameter than the Förster radius. However, biosensors for small metabolites or ions will not become obsolete because those are too small to be tagged with a fluorophore and still perform their biological function. Likewise one cannot not replace biosensors for things such as for mechanical forces or molecular crowding.

H. E. Grecco and P. J. Verveer also hypothesize which new types of measurements may be enabled by the combination of both FRET and nanoscopy. They propose "a fictitious ideal system that could both resolve the spatial distribution of the molecules and the activation status of each molecule by detecting the change in donor/acceptor intensities" and conclude that "In such a system, not only the spatial resolution, but also the dynamic range of the activity measurement would be improved.". This is because the reduction of the detection volume reduces the spatial averaging of the fluorescence emitted from it. If we approximate the detection volume as a spheroid and assume that the equatorial diameter in case of STED is one quarter of the confocal diameter, then a 16-fold reduction of the volume is achieved. This means that the spatial averaging would take place over $1 / 16$ th of the population that was observed before. Experiments using FRET biosensors would especially benefit from reduced spatial averaging as a higher dynamic range could be measured. It would be more probable that the measurements approach the extremal values which the biosensors actually sense.

Furthermore, one could imagine studies of small compartments like endosomes, vesicles, synapses or mitochondria with FRET-STED. In these, FRET could report on protein activity, measure the concentration of small metabolites and ions or to read out parameters such as $\mathrm{pH}$ or mechanical tension. STED would enable the discrimination of separate, but closely adjacent compartments. 
Along these lines, the aim of this thesis is to explore the feasibility of performing STED measurements of samples with FRET pairs. 


\section{Brief literature review: Use of FRET for nanoscopy methods}

\subsection{FRET induced blinking to facilitate SMLM}

SMLM makes use of fluorophore blinking, the rate of which depends on the buffer and the wavelength and intensity of the excitation light. This poses problems for the use of SMLM for live cell imaging. Several publications describe the use of FRET to facilitate SMLM by inducing fluctuations of the fluorescence intensity in various ways:

[25] labeled the structure of interest with the donor molecules and added freely diffusing acceptor molecules. The acceptors transiently quenched the donors when being in close proximity, thus inducing fluorescence fluctuations which enabled superresolution optical fluctuation imaging (SOFI).

Along similar lines, [4] used a FRET pair in DNA-PAINT to achieve fast, backgroundfree superresolution imaging. In one modality, freely diffusing donor-labeled DNA strands bind transiently to acceptor-labeled DNA strands which are fixed to the target structure. In the other modality, both donor- and acceptor-labeled DNA strands diffuse freely and can bind to two sections of the docking strand which is fixed to the target. The advantage of the second modality is that both dyes can be replenished after photobleaching. The disadvantage is that imaging is slower because the probability of both labeled strands binding simultaneously to the docking strand is lower than the probability of single-strand binding.

While studying the photophysics of dark quenchers as acceptors, [56] found that the quenchers induced frequent blinking of the donor molecules. Additionally, photobleaching of the quencher mimicked photoactivation of the donor. They conclude that "the high contrast for switching also introduces these pseudo photoactivatable dyes as possible alternatives for superresolution imaging as any dye can be made photoactivatable in combination with a dark quencher.".

Zong et al. [150] labeled donor quantum dots (QDs) with several Alexa Fluor 647 acceptor molecules. These probes, called FREDEN, showed fluorescence fluctuations ("blinking") in two color channels when excited with 405nm light, making them suitable 
for SMLM. As no special buffer is necessary to induce blinking, they could be suitable for live cell imaging.

\subsection{Enhancing resolution with saturated FRET microscopy}

In 1996, saturated FRET microscopy was proposed by Hänninen, Lehtelä and Hell [53]. In the case of very strong excitation of the donor and extremely efficient energy transfer to the acceptor, FRET will eventually be inhibited because the acceptor will not be able to accept energy while still in the excited state. Thus the donor fluorescence will grow nonlinearly with increasing excitation intensity, similar to two-photon excitation microscopy.

In [104], Schönle, Hänninen and Hell calculated the theoretically attainable resolution increase of saturated FRET microscopy to be 30\% compared to an ideal confocal microscope or a factor of two compared to a two-photon excitation microscope. They also predicted optimal results when using a cluster of five acceptors coupled to one donor.

Deng et al. [31] proposed to improve the spatial resolution of STED microscopy by using fluorophore pairs with extremely high FRET efficiency as fluorescent labels. In addition to the resolution improvement due to saturated FRET, a STED beam could deplete the acceptors in the outer rim of the excitation spot, which in turn would deexcite the donor molecules, thus increasing the resolution of the donor signal even further.

Building on this, Deng et al. showed numerically in [30] that it is important to choose an acceptor with a much longer lifetime than the donor to increase the resolution. Besides, they also point out that one could use a STED beam with a wavelength closer to the acceptor's emission maximum, which would reduce the laser power necessary for efficient depletion [129]. The excitation of the acceptor by the STED beam would not matter because they propose to detect the donor fluorescence.

I would like to point out that the simulations of Deng et al. did not include any noise or background signal. This might significantly compromise the practical implementation of their saturated FRET-STED microscopy, since the donor fluorescence signal which they propose to utilize is of course largely quenched by the energy transfer. As far as I know, no experimental validation of saturated FRET-STED microscopy has been reported so far. 


\subsection{FRET and SMLM provide complementary information}

Two publications report the use of both superresolution imaging and FRET in parallel to gain information about a biological process, but quantitative information about the FRET efficiency was never extracted from the diffraction-unlimited images:

Renz et al. [98] used FRET and molecule counting PALM as complementary methods to determine the subunit stoichiometry of asialoglycoprotein receptors, which form homo- and hetero-oligomers. One of the two subunits was fused to the donor protein, the other to the acceptor protein. The FRET efficiency and the donor-to-acceptor ratio upon oligomerization were calculated and compared to that of a standard construct comprised of one donor and one acceptor. In order to validate the results, a sequential two-color PALM imaging and data analysis method was developed, which yields the relative amount of two photoactivatable proteins. Oligomers of different composition were shown to have distinct ligand specificities.

Winckler et al. [140] developed a two-color uPAINT method to observe the binding of endothelial growth factors (EGF), which were labeled with either donor or acceptor dyes, to EGF receptors and to track the individual EGF receptors afterwards. Binding of EGF to a receptor induces receptor dimerization, which initiates intracellular signaling. FRET taking place on an activated EGF receptor dimer enabled them to distinguish the newly activated receptor dimers, visible in the acceptor channel, from the total EGF receptor population, which is visible in the donor channel.

\subsection{Extracting FRET efficiency from superresolution images}

A thorough search of the literature revealed only one publication in which information about FRET has been extracted from diffraction unlimited images. Stöhr et al. [113] studied graphene exfoliated onto a praseodymium-implanted yttrium aluminum garnet (Pr:YAG) crystal using the quenching of the Pr3+ color center's fluorescence by graphene due to FRET. In order to obtain a diffraction unlimited image, the Pr3+ ions are first pumped into the metastable 1D2 state with 609nm light. Afterwards, the ions in the periphery of the focal spot are further excited by a 532nm doughnut-shaped focus, then decaying quickly into the ground state by emission of UV fluorescence. Last, the ions in the center, which were left in the metastable state, are excited by a 532nm Gaussian beam and their fluorescence is read out to construct the final image. The Pr3+ ions in 
the 1D2 state can also relax into the ground state when transferring their energy to the graphene above. Thus, the presence of graphene can be inferred from the shortened fluorescence lifetime of Pr3+. This special system allows for the observation of FRET in diffraction-unlimited, time-resolved images because of the $210 \mu$ s long lifetime of the 1D2 state. It leaves a sufficient number of ions in the metastable, excitable state after the action of the pump beam and doughnut beam to still observe the accelerated decay due to FRET. If the donors had a shorter lifetime, most of them would have decayed already before the start of the readout time gate, not leaving enough signal to analyze.

In conclusion, this kind of superresolution measurement is not possible with organic fluorophores, because they do not possess such a metastable fluorescent state. The lifetime of organic dyes is less than 10ns. However, the example of [113] shows a possible solution for combining STED and lifetime FRET, namely the use of donors with very long lifetimes, such as lanthanide ion complexes. Rajendran et al. describe in 2013 [97] and 2015 [96] the use of lanthanide ion complexes as donors to image protein-protein interactions in live cells. Time-gated detection of the fluorescence decay after the action of the STED pulse on the donors could reveal the accurate FRET efficiency, when used together with acceptors that are not bleached by such a STED beam with a relatively short wavelength. 


\section{Part III}

\section{Simulations}

In my experiments with FRET pairs, their FRET efficiency, calculated from spectral intensity ratios, appeared to depend on the applied STED intensity and the delay between excitation and STED pulses. Usually in STED imaging experiments, the STED pulse delay is optimized once and then left unchanged for similar samples. However, the setting could be different on different microscopes, which would complicate the comparison of data sets. The STED intensity is usually balanced in each sample between the required spatial resolution and the photostability of the fluorophores.

Simulations were performed to study the influence of these individual parameters and to investigate whether this observed dependence was a measurement artifact or whether it can be explained as an interference of stimulated emission with the FRET process. At first, a simple model was chosen to explore if only basic electronic state transitions were involved or if additional processes would need to be considered to explain the experimental data. Further simulations were performed to find out if continuous wave or pulsed STED would be more suitable for FRET experiments and what roles the donor's lifetime and quantum yield play.

Simulations of the ground and excited state population dynamics of a donor and an acceptor molecule upon direct excitation of the molecules, spontaneous and stimulated emission and energy transfer between them are shown in this part. Only the ground state $S_{0}$ and the first excited state $S_{1}$ of each molecule and the transitions in between are considered, as depicted in Fig. 9.1. Electrons in higher excited singlet states vibrationally relax back to the first excited state very quickly, as explained in section 2.1. Therefore, higher excited singlet states are omitted in the simulations. Intersystem crossing from the first excited state to the triplet state is also excluded, because the probability is low. Furthermore, the lifetime of the electron in the triplet state is much longer than the duration of the events simulated here. Besides, for most molecules the energy levels and transition rates of the triplet state are not characterized. 


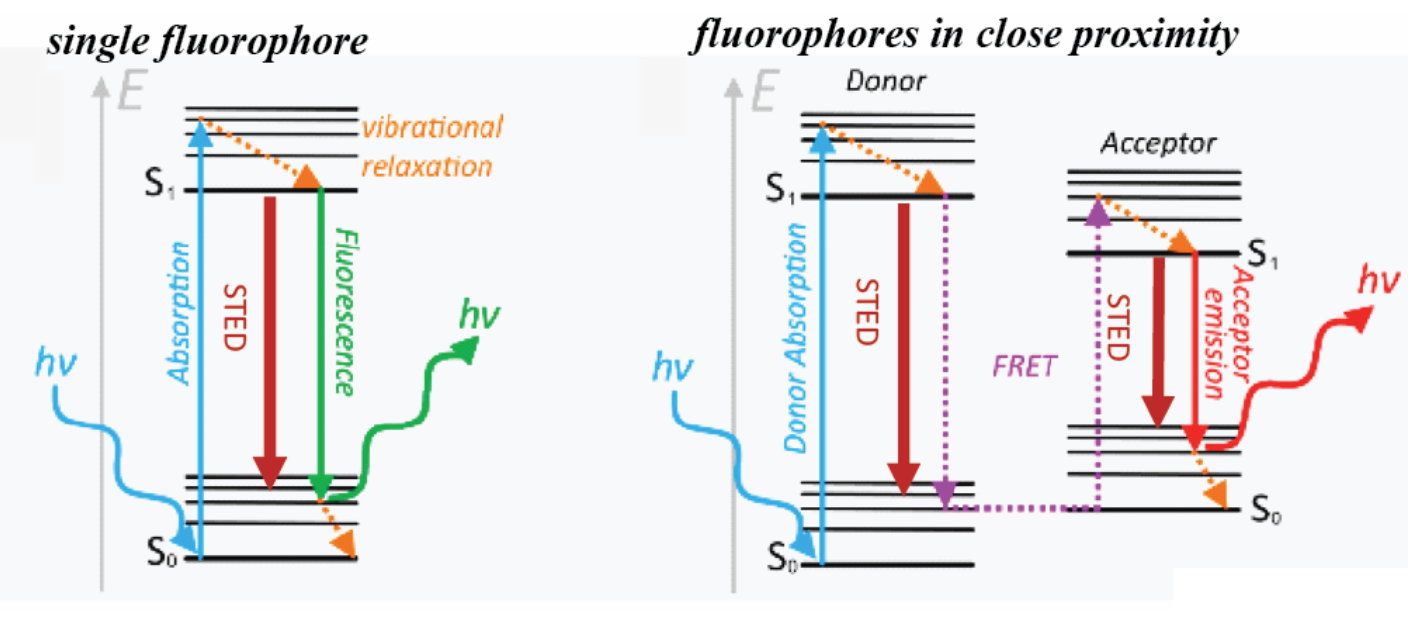

Fig. 9.1: Jablonski diagram showing electronic transitions between ground and first excited state upon absorption, spontaneous and stimulated emission, for a single fluorophore and for two fluorophores between which FRET occurs. Figure adapted from [54].

Bleaching of fluorophores due to photochemical reactions occurs mostly when the electron is transferred to higher excited singlet states or triplet states which were not modeled here. It is very relevant when one considers an ensemble of fluorophores, e.g. in imaging. In a single pair of molecules studied here, bleaching to a non-fluorescent and non-absorbing product would simply abolish the energy transfer. Photoconversion of one partner to a product with changed absorption and emission spectra would be more problematic, as it would cause the spectral overlap integral and thus the FRET efficiency to change. This process is not considered in the simulations, but it is further examined in chapter 22 .

Simulations were performed by numerically solving the rate equation system containing the transition rates corresponding to absorption, spontaneous and stimulated emission and FRET, iteratively for small time steps. Excitation pulses with a Gaussian temporal profile were always used. In the first chapter of this part, stimulated emission by a continuous wave (CW) STED beam is described, whereas in the second chapter, stimulated emission with laser pulses is studied. The rate equation system is not solvable analytically for either case, as will be discussed in section 10.1. In each chapter, the result of changing the FRET rate or the STED intensity is investigated, which corresponds to different experimental settings. Additionally, the influence of varying the delay between excitation and STED pulses is examined, which is an important experimental parameter to choose.

The simulation results are visualized either by plotting the excited state population over time, i.e. lifetime histograms, or by mapping its spatial distribution, which corresponds to line profiles, in order to facilitate the subsequent comparison to experimental data. The acceptor's fluorescence emission is always of more interest here because it forms 
the diffraction unlimited image. The donor is not depleted efficiently, so its emission remains diffraction limited.

Lifetime histograms measured for different FRET rates, STED intensities and pulse delays are shown in chapter 18 and fits to intensity distributions (PSF) in chapter 19. The spectral FRET efficiency was calculated from simulated data sets with different parameters and compared to experimentally determined spectral FRET efficiency in chapter 20. 



\section{Combining FRET with CW-STED}

Diffraction unlimited imaging with CW-STED lasers and pulsed excitation has previously been shown to perform well, especially if time gated detection is implemented [128]. Therefore, simulations of FRET pairs under CW-STED are conducted here.

\subsection{System of rate equations for FRET with CW-STED}

In this chapter, a donor-acceptor pair is studied, which absorbs an excitation pulse, and can subsequently spontaneously emit fluorescence or resonantly transfer energy and is simultaneously depleted by a CW-STED beam. The following equations ${ }^{1}$ describe the ground state $S_{0}$ population dynamics of the donor molecule

$$
\frac{\partial N_{0}^{D}}{\partial t}=-k_{\mathrm{exc}}^{D} \cdot N_{0}^{D}(t) \cdot I_{\mathrm{exc}}(r, t)+\left(k_{\mathrm{em}}^{D}+k_{\mathrm{FRET}}\right) \cdot N_{1}^{D}(t)+k_{\mathrm{STED}}^{D} \cdot I_{\mathrm{STED}}(r) \cdot N_{1}^{D}(t)
$$

and of the acceptor molecule:

$$
\frac{\partial N_{0}^{A}}{\partial t}=-k_{\mathrm{exc}}^{A} \cdot N_{0}^{A}(t) \cdot I_{\mathrm{exc}}(r, t)+k_{\mathrm{em}}^{A} \cdot N_{1}^{A}(t)-k_{\mathrm{FRET}} \cdot N_{1}^{D}(t)+k_{\mathrm{STED}}^{A} \cdot I_{\mathrm{STED}}(r) \cdot N_{1}^{A}(t)
$$

The corresponding equations for calculating the first excited singlet state $S_{1}$ population dynamics of the donor

$$
\frac{\partial N_{1}^{D}}{\partial t}=k_{\mathrm{exc}}^{D} \cdot N_{0}^{D}(t) \cdot I_{\mathrm{exc}}(r, t)-\left(k_{\mathrm{em}}^{D}+k_{\mathrm{FRET}}\right) \cdot N_{1}^{D}(t)-k_{\mathrm{STED}}^{D} \cdot I_{\mathrm{STED}}(r) \cdot N_{1}^{D}(t)
$$

and of the acceptor are:

$$
\frac{\partial N_{1}^{A}}{\partial t}=k_{\mathrm{exc}}^{A} \cdot N_{0}^{A}(t) \cdot I_{e x c}(r, t)-k_{\mathrm{em}}^{A} \cdot N_{1}^{A}(t)+k_{\mathrm{FRET}} \cdot N_{1}^{D}(t)-k_{\mathrm{STED}}^{A} \cdot I_{\mathrm{STED}}(r) \cdot N_{1}^{A}(t)
$$

In each case the superscript $\mathrm{D}$ refers to the donor, and the superscript $\mathrm{A}$ to the acceptor molecule. The subscript 0 denotes ground state, and the subscript 1 the first excited state. The subscript "exc" stands for excitation of the electron upon absorption, and the subscript "em" stands for spontaneous emission.

${ }^{1}$ Compare to [30]. 
The excitation beam has a Gaussian intensity profile of width (s.d.) $\sigma_{\text {exc }}$ and peak height $I_{\text {exc }}$

$$
a_{\mathrm{exc}}(r)=I_{\mathrm{exc}} \cdot \exp \left(-\frac{r^{2}}{2 \cdot \sigma_{\mathrm{exc}}^{2}}\right)
$$

and is pulsed with a Gaussian temporal profile of width $w_{\text {exc }}$ :

$$
I_{\mathrm{exc}}(r, t)=a_{\mathrm{exc}}(r) \cdot \exp \left(-\frac{\left(t-t_{\mathrm{p}, \mathrm{exc}}\right)^{2}}{2 \cdot w_{\mathrm{exc}}^{2}}\right)
$$

The excitation pulse is delayed by $t_{\mathrm{p} \text {,exc }}$ with respect to the start of the simulation. The intensity profile of the STED focus is torus-shaped with width $\sigma_{\mathrm{STED}}$ :

$$
I_{\mathrm{STED}}(r)=I_{\mathrm{max}} \cdot \exp \left(-\frac{r^{2}}{2 \cdot \sigma_{\mathrm{STED}}^{2}}\right) \cdot r^{2}
$$

\section{Rate constants}

For performing the simulations, the following rate constants ${ }^{2}$ were chosen:

$$
\begin{array}{lll}
k_{\mathrm{exc}}^{D}=0.15 \frac{\mathrm{m}^{2}}{\mathrm{~ns} \cdot \mathrm{W}} & k_{\mathrm{em}}^{D}=1 / 3.8 \mathrm{~ns}=0.26 \mathrm{~ns}^{-1} & k_{\mathrm{STED}}^{D}=0.001 \frac{\mathrm{m}^{2}}{\mathrm{~ns} \cdot \mathrm{W}} \\
k_{\mathrm{exc}}^{A}=0.02 \frac{\mathrm{m}^{2}}{\mathrm{~ns} \cdot \mathrm{W}} & k_{\mathrm{em}}^{A}=1 / 3.3 \mathrm{~ns}=0.30 \mathrm{~ns}^{-1} & k_{\mathrm{STED}}^{A}=0.015 \frac{\mathrm{m}^{2}}{\mathrm{~ns} \cdot \mathrm{W}}
\end{array}
$$

The lifetimes of the fluorophores used later in experiments are specified as donor $\tau_{\text {Atto532 }}=3.8 \mathrm{~ns}$ and acceptor $\tau_{\text {Star635P }}=3.3 \mathrm{~ns}$ by the manufacturers. The transition rate and the lifetime are related by $\tau=1 / k$, as stated in theory section 2.1. The characteristic lifetime is defined as the point in time at which the intensity dropped to $1 / \mathrm{e}=37 \%$. The rates for excitation and stimulated emission were chosen such that the simulated lifetime histograms correspond well to the measured ones.

The transfer rates used in the simulations, stated in table 10.1, are the same as expected for the DNA constructs used later in experiments, which are given in table 15.1.

The FRET efficiency $E_{\mathrm{FRET}}$, transfer rate $k_{\mathrm{FRET}}$ and the lifetime of the donor in the absence $\tau_{\mathrm{D}}$ and presence $\tau_{\mathrm{DA}}$ of the acceptor are related as follows:

$$
E_{\mathrm{FRET}}=1-\frac{\tau_{\mathrm{DA}}}{\tau_{\mathrm{D}}}=\frac{k_{\mathrm{FRET}}}{\frac{1}{\tau_{\mathrm{D}}}+k_{\mathrm{FRET}}}
$$

\footnotetext{
${ }^{2}$ The intensities $I_{\mathrm{exc}}(r, t)$ and $I_{\mathrm{STED}}(r)$ are given in units of $\left[\frac{\mathrm{W}}{\mathrm{m}^{2}}\right]$. Thus $I_{\mathrm{exc}}$ is also given in $\left[\frac{\mathrm{W}}{\mathrm{m}^{2}}\right]$ and $I_{\max }$ in $\left[\frac{\mathrm{W}}{\mathrm{m}^{4}}\right]$.
} 


\begin{tabular}{|l|l|l|l|l|l|}
\hline$E_{\text {FRET }}$ & 0.93 & 0.69 & 0.34 & 0.13 & 0.05 \\
\hline$\tau_{\text {DA }}(\mathrm{ns})$ & 0.27 & 1.18 & 2.51 & 3.31 & 3.61 \\
\hline$k_{\text {FRET }}(1 / \mathrm{ns})$ & 3.50 & 0.59 & 0.14 & 0.04 & 0.01 \\
\hline
\end{tabular}

Tab. 10.1: FRET efficiencies, corresponding transfer rates and lifetimes of the donor fluorophore used in simulations. The FRET efficiencies are the same as expected for the DNA constructs used in later experiments, which are stated in table 15.1.

When the FRET efficiency and original donor lifetime are known, the decreased lifetime and the transfer rate can be calculated:

$$
\begin{gathered}
\tau_{\mathrm{DA}}=\left(1-E_{\mathrm{FRET}}\right) \tau_{\mathrm{D}} \\
k_{\mathrm{FRET}}=\frac{E_{\mathrm{FRET}}}{(1-E) \tau_{\mathrm{D}}}=\frac{E_{\mathrm{FRET}}}{\tau_{\mathrm{DA}}}
\end{gathered}
$$

When changing the STED intensity or pulse delay in simulations, the FRET efficiency was kept constant.

The parameters describing the laser beams are listed in subsection 11.1.

\section{Solvability of the equation system}

The rate equation system given above was studied with the software Mathematica (Wolfram). It is solvable if excitation and STED intensity are constant over time. If even one intensity has an explicit time dependence, the equation system is not solvable. This is the case even for a simple, derivable function such as $I_{\text {exc }}(t)=\sin ^{2}(t)$. Therefore, simulations with laser pulses had to be carried out numerically. Furthermore, it means that no explicit analytical fit function for measured lifetime histograms is available, which could have been used to deduce the FRET efficiency.

In order to circumvent this problem in case of pulsed STED, one could estimate the excitation and depletion of the fluorophores by the laser pulses and use them as initial conditions. This way, the time dependent terms are removed, so the rate equations can be solved analytically to yield the following expressions for the detected intensities in donor, acceptor and FRET channel. The equations were adapted from the supplementary information of [23]:

$$
\begin{aligned}
& I_{\text {Aex }}^{\text {Aem }}=N_{1}^{\mathrm{A}}(t) \cdot k_{\mathrm{em}}^{\mathrm{A}}=N_{\text {Achan }}(0) \cdot \exp \left(-\frac{t}{\tau_{\mathrm{A}}}\right) \cdot \frac{Q Y_{\mathrm{A}}}{\tau_{\mathrm{A}}} \\
& I_{\text {Dex }}^{\text {Dem }}=\left(N_{\mathrm{D}}(0) \cdot \exp \left(-\frac{t}{\tau_{\mathrm{D}}}\right)+N_{\mathrm{D}, \mathrm{FRET}}(0) \cdot \exp \left(-\frac{t}{\tau_{\mathrm{DA}}}\right)\right) \cdot \frac{Q Y_{\mathrm{D}}}{\tau_{\mathrm{D}}} \\
& I_{\mathrm{Dex}}^{\text {Aem }}=x \cdot N_{\mathrm{D}, \mathrm{FRET}}(0) \cdot \frac{\tau_{\mathrm{D}}-\tau_{\mathrm{DA}}}{\left(\tau_{\mathrm{A}}-\tau_{\mathrm{DA}}\right) \tau_{\mathrm{D}}} \cdot\left(\exp \left(-\frac{t}{\tau_{\mathrm{A}}}\right)-\exp \left(-\frac{t}{\tau_{\mathrm{DA}}}\right)\right) \cdot Q Y_{\mathrm{A}}+y \cdot I_{\mathrm{Aex}}^{\mathrm{Aem}}+x \cdot I_{\mathrm{Dex}}^{\mathrm{Dem}}
\end{aligned}
$$


These equations could be used to perform a global tailfit of the obtained lifetime histograms in the donor, acceptor and FRET channel to deduce $\tau_{\mathrm{DA}}$ and therewith $E_{\mathrm{FRET}}$. The drawback is that by fitting only after excitation and STED pulse have passed, the first approximately $1.1 \mathrm{~ns}$ of the histogram would be neglected. This corresponds to the duration of the most efficient FRET processes, which are stated as $\tau_{\mathrm{DA}}$ in table 10.1.

In case of CW-STED the intensity is constant over time, therefore the depletion can also be included in the equations to perform a global tailfit after the excitation pulse:

$$
\begin{aligned}
I_{\mathrm{Aex}}^{\mathrm{Aem}}= & N_{\mathrm{Achan}}(0) \cdot \exp \left(-\frac{t}{\tau_{\mathrm{A}}+\tau_{\mathrm{A}, \mathrm{STED}} \cdot I_{\mathrm{STED}}(r)}\right) \cdot \frac{Q Y_{\mathrm{A}}}{\tau_{\mathrm{A}}} \\
I_{\mathrm{Dex}}^{\mathrm{Dem}}= & N_{\mathrm{D}}(0) \cdot \exp \left(-\frac{Q Y_{\mathrm{D}}}{\tau_{\mathrm{D}}+\tau_{\mathrm{D}, \mathrm{STED}} \cdot I_{\mathrm{STED}}(r)}\right) \cdot \frac{t}{\tau_{\mathrm{D}}} \\
& +N_{\mathrm{D}, \mathrm{FRET}}(0) \cdot \exp \left(-\frac{t}{\tau_{\mathrm{DA}}+\tau_{\mathrm{DA}, \mathrm{STED}} \cdot I_{\mathrm{STED}}(r)}\right) \cdot \frac{Q Y_{\mathrm{D}}}{\tau_{\mathrm{D}}} \\
I_{\mathrm{Dex}}^{\mathrm{Aem}}= & y \cdot I_{\mathrm{Aex}}^{\mathrm{Aem}}+x \cdot I_{\mathrm{Dex}}^{\mathrm{Dem}}+x \cdot N_{\mathrm{D}, \mathrm{FRET}}(0) \cdot \frac{\tau_{\mathrm{D}}-\tau_{\mathrm{DA}}}{\left(\tau_{\mathrm{A}}-\tau_{\mathrm{DA}}\right) \tau_{\mathrm{D}}} \cdot Q Y_{\mathrm{A}} \\
& \times\left(\exp \left(-\frac{t}{\tau_{\mathrm{A}}+\tau_{\mathrm{A}, \mathrm{STED}} \cdot I_{\mathrm{STED}}(r)}\right)-\exp \left(-\frac{t}{\tau_{\mathrm{DA}}-\tau_{\mathrm{DA}, \mathrm{STED}} \cdot I_{\mathrm{STED}}(r)}\right)\right)
\end{aligned}
$$

\subsection{Changing the FRET rate under CW-STED light}

This section explores the effect of the FRET rate under otherwise unchanged conditions. Simulated lifetime histograms of donor and acceptor are shown in Fig. 10.1. As expected, the donor lifetime decreases with increasing FRET efficiency and hardly any stimulated depletion is visible. On the other hand, the acceptor lifetime histogram shows increased photon counts right after excitation, which deviate strongly from a monoexponential decay. For high transfer rates, the histogram's peak is high and decreases sharply, because transfer happens fast and the donor returns very soon to the ground state. For low transfer rates, the peak is lower with a gentler slope, as the transfer happens over a longer period of time.

Simulated line profiles, i.e. the spatial distribution of the donor's and acceptor's excited state population, are shown in the lower half of Fig. 10.1. One can clearly see in the left plots that an increasing energy transfer rate leads to increasing acceptor intensity and simultaneously decreasing donor intensity, as expected. In the normalized distributions on the right hand side, it is evident that at the same STED intensity, all acceptors have nearly same FWHM, which is smaller than the donor's FWHM, as expected due to stimulated emission depletion. 

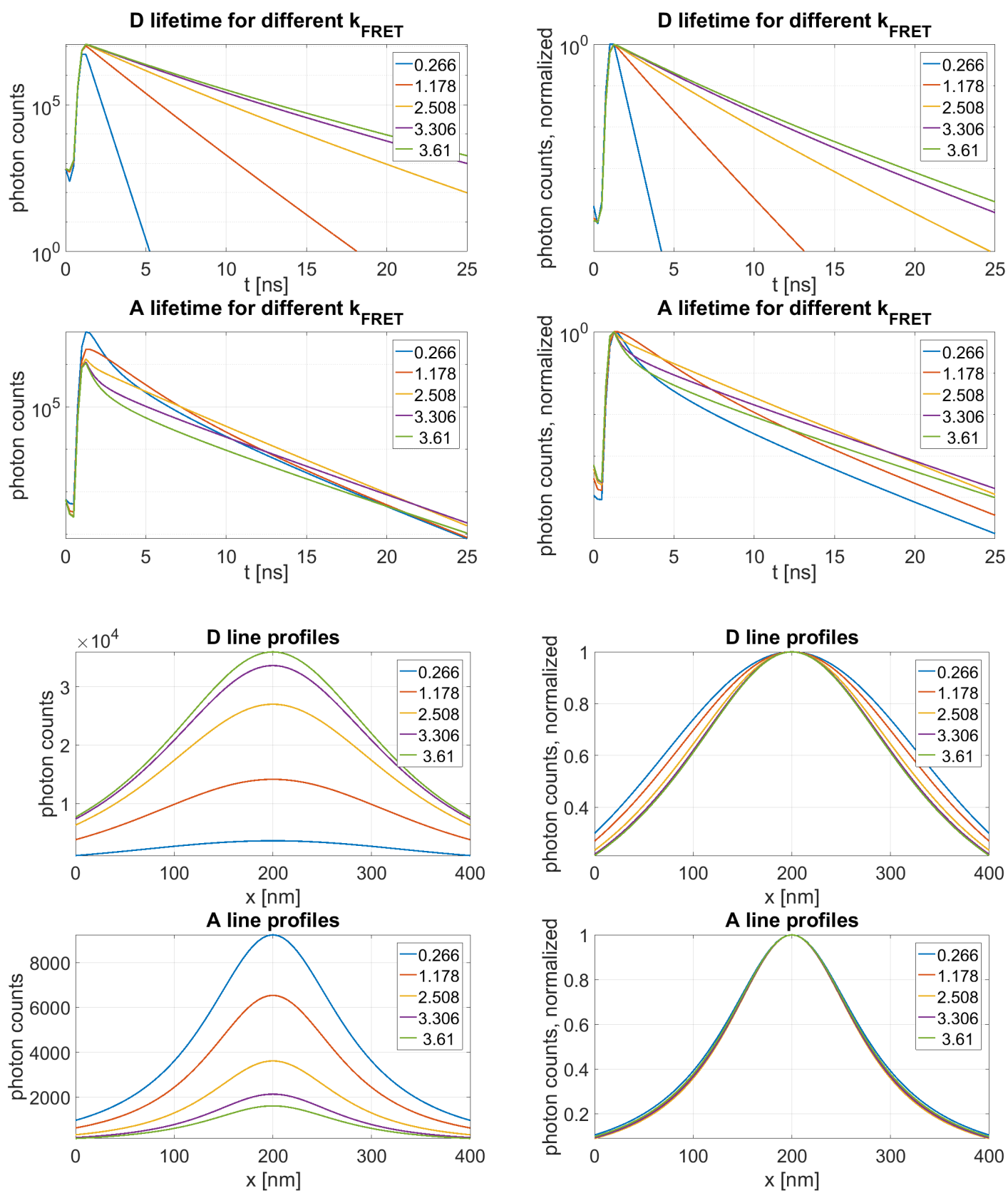

Fig. 10.1: Top: Simulations of the excited state population over time, i.e. lifetime histograms, of a donor and acceptor with different transfer rates under CW-STED light of constant intensity. (semi-log plots) Bottom: Line profiles through the center of the spatial distribution of the excited state populations. The legend states the lifetime of the donor in the presence of the acceptor in ns, refer to table 10.1. The data in the right plots is the same as in the left plots, only normalized. 


\subsection{Changing the CW-STED intensity at constant FRET rate}

In the simulations presented in this section, the transfer efficiency was kept constant at $60 \%$, while the intensity of the CW-STED beam was increased. In the lifetime histograms depicted in Fig. 10.2, the effect of continuous depletion is clearly visible as an initial decrease of the acceptor excited state population, which is steeper the higher the STED intensity.

The associated line profiles in Fig. 10.2 show that a higher STED intensity slightly decreases the FWHM and the total fluorescence intensity of both donor and acceptor as expected. The acceptor's FWHM is narrowed by stimulated emission, but not as much as one might expect when comparing to line profiles obtained under pulsed STED as shown in Fig. 11.1. This is because a four to five times higher CW-STED power or conversely lower pulse energy are needed to achieve the same resolution as with pulsed STED ([139], [73]). 

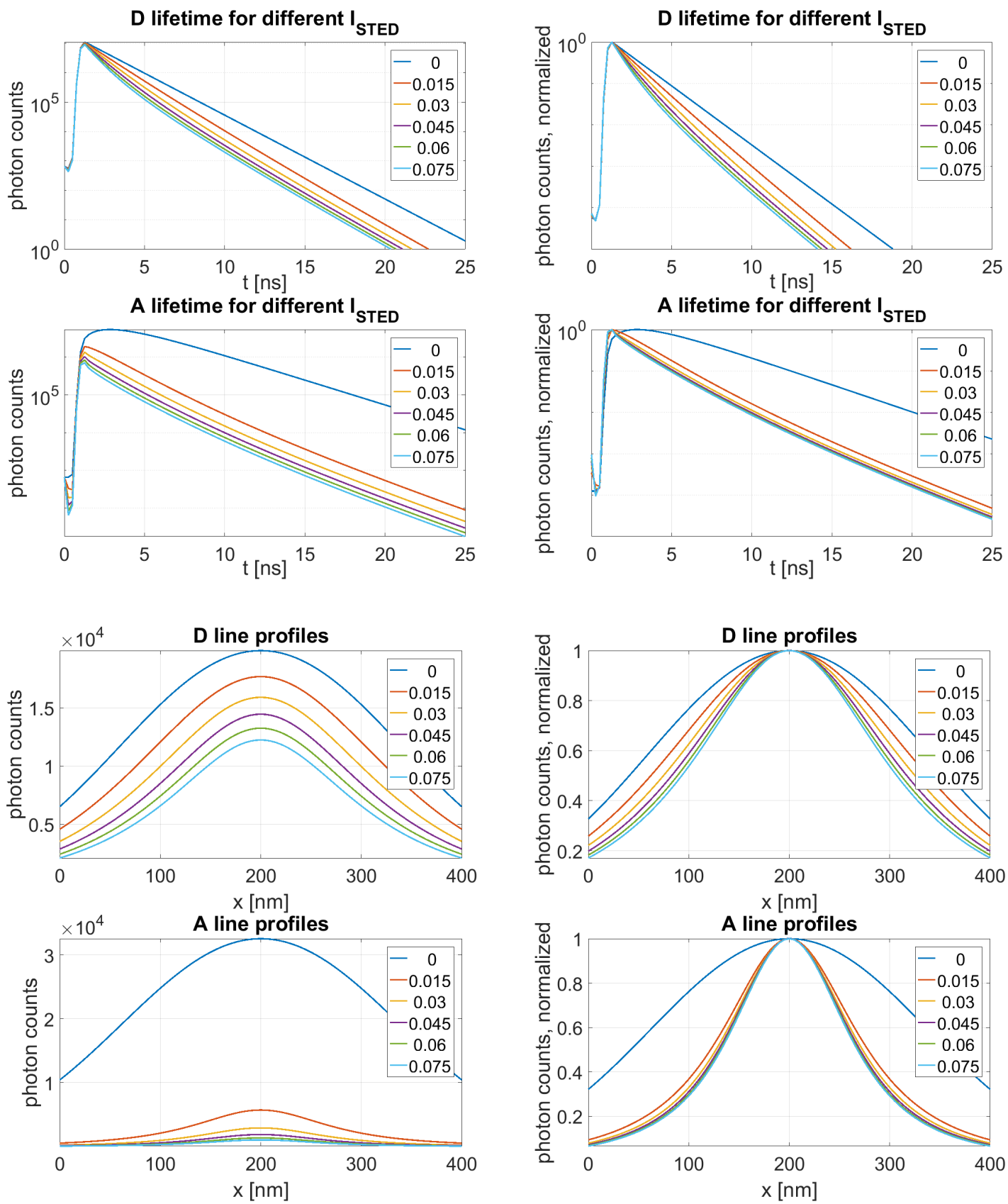

Fig. 10.2: Top half: Simulation of the excited state dynamics of a FRET pair with $60 \%$ transfer efficiency under CW-STED with increasing intensity. (semi-log plots) Bottom half: Simulated line profiles of the same FRET pair. The legend states the STED peak intensity in arbitrary units. The data on the right hand side is the same as in the left plots, only normalized. 



\section{Combining FRET with pulsed STED}

\subsection{System of rate equations for pulsed STED}

This section investigates the influence of a short STED laser pulse on a FRET pair. The rate equations below describe the excitation of donor electrons to the $S_{1}$ state after absorption of a photon, followed by either spontaneous or stimulated emission or return to the ground state after energy transfer to the acceptor. The acceptor can be excited directly by absorption or via FRET and can afterwards release energy by spontaneous or stimulated emission. The following equations govern the ground state $S_{0}$ population of the donor

$$
\frac{d N_{0}^{D}}{d t}=-k_{\mathrm{exc}}^{D} \cdot N_{0}^{D}(t) \cdot I_{\mathrm{exc}}(r, t)+\left(k_{\mathrm{em}}^{D}+k_{\mathrm{FRET}}\right) \cdot N_{1}^{D}(t)+k_{\mathrm{STED}}^{D} \cdot I_{\mathrm{STED}}(r, t) \cdot N_{1}^{D}(t)
$$

and of the acceptor:

$$
\frac{d N_{0}^{A}}{d t}=-k_{\mathrm{exc}}^{A} \cdot N_{0}^{A}(t) \cdot I_{\mathrm{exc}}(r, t)+k_{\mathrm{em}}^{A} \cdot N_{1}^{A}(t)-k_{\mathrm{FRET}} \cdot N_{1}^{D}(t)+k_{\mathrm{STED}}^{A} \cdot I_{\mathrm{STED}}(r, t) \cdot N_{1}^{A}(t)
$$

The equations below describe the first excited singlet state $S_{1}$ population of the donor

$$
\frac{d N_{1}^{D}}{d t}=k_{\mathrm{exc}}^{D} \cdot N_{0}^{D}(t) \cdot I_{\mathrm{exc}}(r, t)-\left(k_{\mathrm{em}}^{D}+k_{\mathrm{FRET}}\right) \cdot N_{1}^{D}(t)-k_{\mathrm{STED}}^{D} \cdot I_{\mathrm{STED}}(r, t) \cdot N_{1}^{D}(t)
$$

and of the acceptor:

$$
\frac{d N_{1}^{A}}{d t}=k_{\mathrm{exc}}^{A} \cdot N_{0}^{A}(t) \cdot I_{\mathrm{exc}}(r, t)-k_{\mathrm{em}}^{A} \cdot N_{1}^{A}(t)+k_{\mathrm{FRET}} \cdot N_{1}^{D}(t)-k_{\mathrm{STED}}^{A} \cdot I_{\mathrm{STED}}(r, t) \cdot N_{1}^{A}(t)
$$

In each case the superscript $\mathrm{D}$ refers to the donor, and the superscript $\mathrm{A}$ to the acceptor molecule. The subscript 0 denotes ground state, and the subscript 1 the first excited state. The subscript "exc" stands for excitation of the electron upon absorption, and the subscript "em" stands for spontaneous emission. 
The equations describing the Gaussian intensity profile of the excitation beam as well as its Gaussian temporal profile are given in the previous chapter 10.1. The intensity profile of the STED focus is torus-shaped with peak intensity $I_{\max }$ :

$$
a_{\mathrm{STED}}(r)=I_{\max } \cdot \exp \left(-\frac{r^{2}}{2 \cdot \sigma_{\mathrm{STED}}^{2}}\right) \cdot r^{2}
$$

The STED pulse also has Gaussian temporal profile with width $w_{\text {STED }}$ and delay $t_{\mathrm{P}, \mathrm{STED}}$ :

$$
I_{\mathrm{STED}}(r, t)=a_{\mathrm{STED}}(r) \cdot \exp \left(-\frac{\left(t-t_{\mathrm{p}, \mathrm{STED}}\right)^{2}}{2 \cdot w_{\mathrm{STED}}^{2}}\right)
$$

It follows that the relative delay between excitation and STED pulse is given by $t_{\mathrm{p}, \mathrm{STED}}-$ $t_{\mathrm{p}, \text { exc. }}$

\section{Laser beam parameters}

In the simulations, the following parameter values were chosen to match the experimental conditions:

$$
\begin{array}{lll}
t_{\mathrm{p}, \text { exc }}=1 \mathrm{~ns} & w_{\mathrm{exc}}=0.3 \mathrm{~ns} & \sigma_{\text {exc }}=127 \mathrm{~nm} \\
t_{\mathrm{p}, \mathrm{STED}}=1.5 \mathrm{~ns} & w_{\mathrm{STED}}=0.8 \mathrm{~ns} & \sigma_{\mathrm{STED}}=160 \mathrm{~nm}
\end{array}
$$

The standard deviation (s.d.) $\sigma$ is related to the FWHM by: FWHM $=2 \cdot \sqrt{2 \cdot \ln (2)} \cdot \sigma \approx$ $2.35 \cdot \sigma$.

A STED intensity of $I_{\max }=0.015 a$.u. was set for CW-STED and a peak intensity of $I_{\max }=0.075 \mathrm{a}$.u. was set for pulsed STED. The duty cycle of the STED laser with $40 \mathrm{MHz}$ repetition rate, corresponding to $25 \mathrm{~ns}$ pulse intervals, is

$$
\frac{\delta t}{T}=\frac{0.8 \mathrm{~ns}}{25 \mathrm{~ns}}=0.032=\frac{P_{\mathrm{avr}}}{P_{\text {peak }}}
$$

This means that a peak intensity of $I_{\max }=0.075$ a.u. is equivalent to an average intensity of 0.0024 a.u., which is 6.25 times lower than the chosen average CW-STED intensity.

For these simulations, the same values of the rate constants for excitation, emission and STED were used which are listed in subsection 10.1. 


\subsection{Changing the FRET rate under pulsed STED light}

In the simulations shown in this section, the transfer rate is changed while the other parameters are kept constant. In the simulated lifetime histograms in Fig. 11.1, the stimulated emission depletion is clearly visible as a sharp, short decrease immediately after the excitation peak. The donor fluorescence decays monoexponentially after the action of the STED pulse. As expected, the donor lifetime decreases with increasing transfer efficiency. In the acceptor lifetime histogram, a second peak or shoulder appears after depletion due to ongoing excitation of the acceptor via FRET. The lower the FRET rate, the lower and broader and slightly later the second peak. The last part of the acceptor histogram approximately follows a monoexponential decay, which is governed by the acceptor's lifetime.

In the line profiles on the left hand side in Fig. 11.1, one can clearly see the decrease in donor fluorescence and corresponding increase in acceptor fluorescence due to the energy transfer. The same data is shown on the right hand side, only normalized. There, the significant decrease of the acceptor's FWHM due to stimulated emission depletion is visible, compared to the donor's nearly unaffected FWHM. Notably, the steepness of the line profiles differs. In the case of medium FRET efficiency, a major amount of energy is transferred to the acceptor after it has been depleted by the STED pulse. Therefore, the acceptor can subsequently emit fluorescence again, giving rise to a broader PSF. On the other hand, if the FRET efficiency is very high, most energy is transferred before or during the action of the STED pulse, thus most fluorescence is emitted by the acceptor within the confines of the STED torus. This phenomenon will be discussed in more detail in section 11.5.

\subsection{Changing STED intensity at constant FRET rate}

This section explores the effect of increasing the STED intensity, while the FRET rate and other parameters are kept constant. In the simulated lifetime histograms in Fig. 11.2, the short fluorescence decrease directly after excitation, which is due to stimulated emission depletion, becomes steeper and deeper with increasing STED pulse intensity. The acceptor is affected more strongly than the donor, as expected. The minimum is reached at the same time point in every case, because the STED pulse width and delay are constant. 

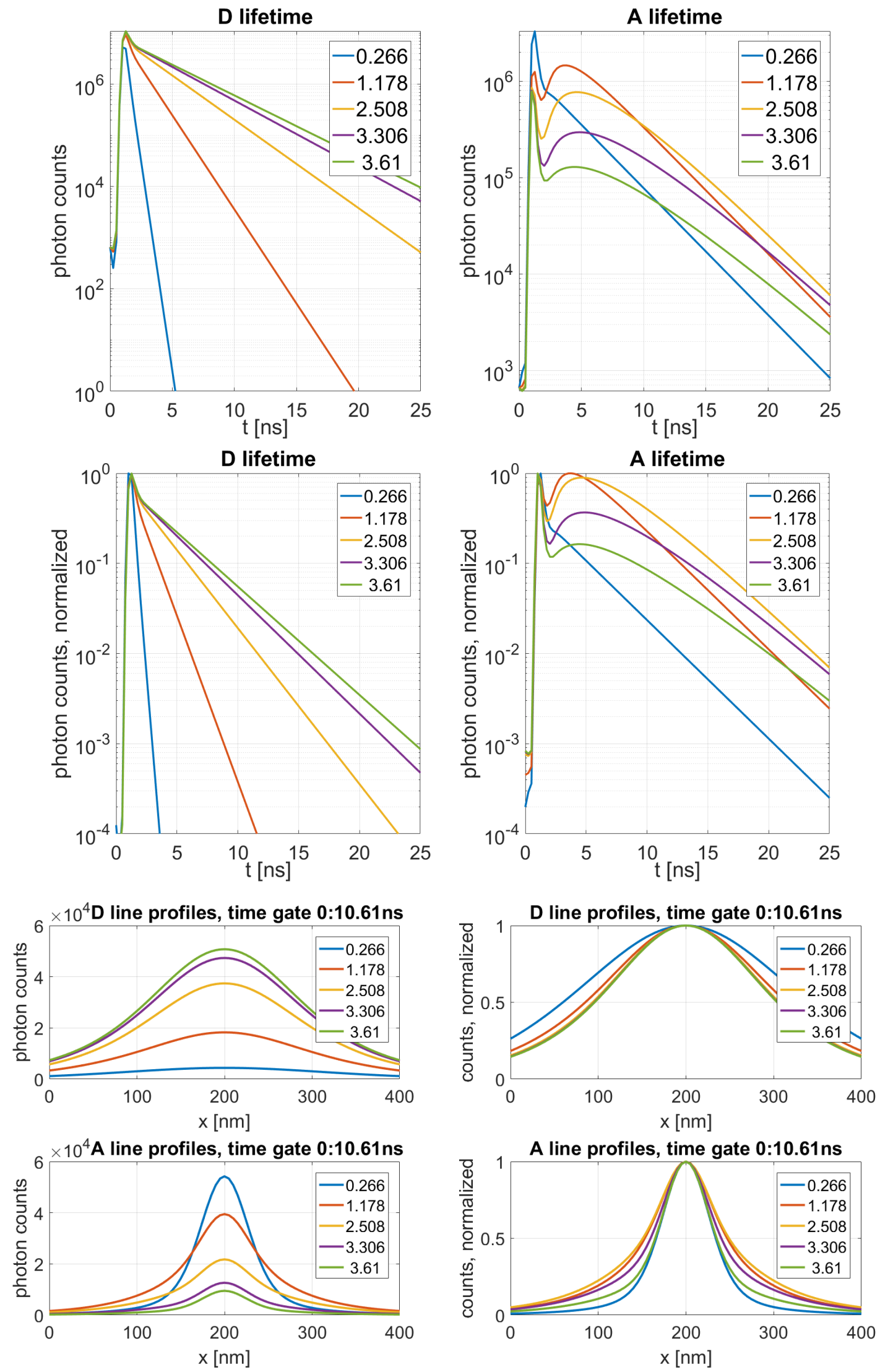

Fig. 11.1: Top half: Simulation of the excited state dynamics of FRET pairs with different transfer rates under pulsed STED light of constant intensity. (semi-log plots) Bottom half: Simulated line profiles of FRET pairs with different transfer rates. The legends state the lifetime of the donor in ns, as listed in table 10.1. The histograms in the lower panel show the same data as above, only normalized. 

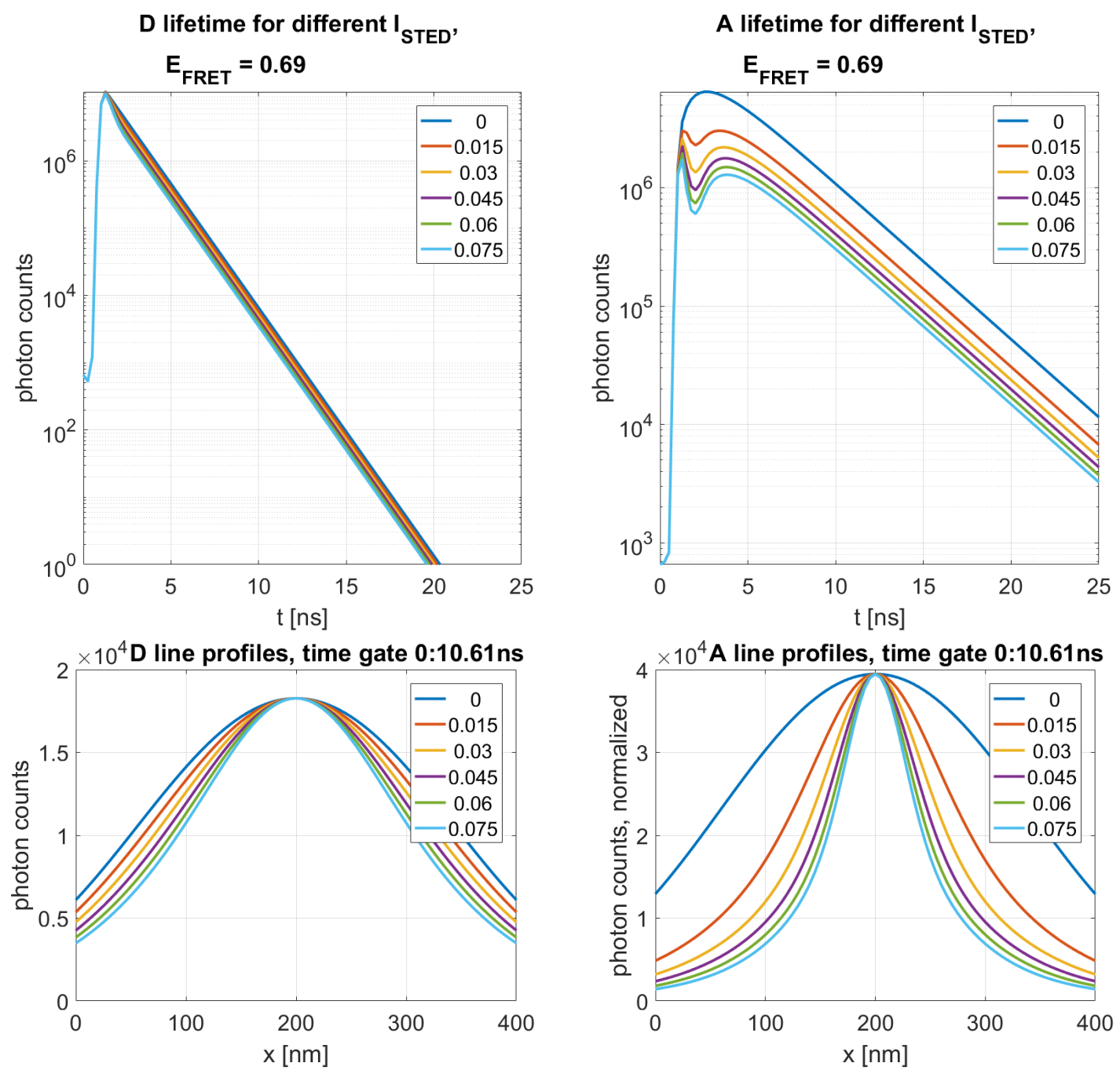

Fig. 11.2: Simulation of the excited state dynamics of a FRET pair with $69 \%$ transfer efficiency under pulsed STED light of increasing intensity. Top: Simulated lifetime histograms of donor and acceptor. (semi-log plots) Bottom: Simulated line profiles. The legend states the maximum STED intensity in arbitrary units.

It is evident that the FWHM of the line profiles in Fig. 11.2 decreases with increasing STED intensity as expected. Again, the acceptor is depleted more efficiently due to its $15 \times$ higher STED rate, thus reaching a better spatial resolution.

\subsection{Changing STED delay at constant FRET rate}

In this section, the consequences of changing the relative delay between excitation pulse and STED pulse are analyzed. In the lifetime histograms in Fig. 11.3, the temporal shift of the stimulated emission depletion relative to the excitation pulse is evident, especially in case of the acceptor. One can see the ratio of both peaks changing: either more photons are emitted before or after the action of the STED pulse. This results in different acceptor line profile shapes, as depicted in the lower right panel of Fig. 11.3. 
D lifetime for different STED delay, $\mathrm{E}_{\text {FRET }}=\mathbf{0 . 6 9}$
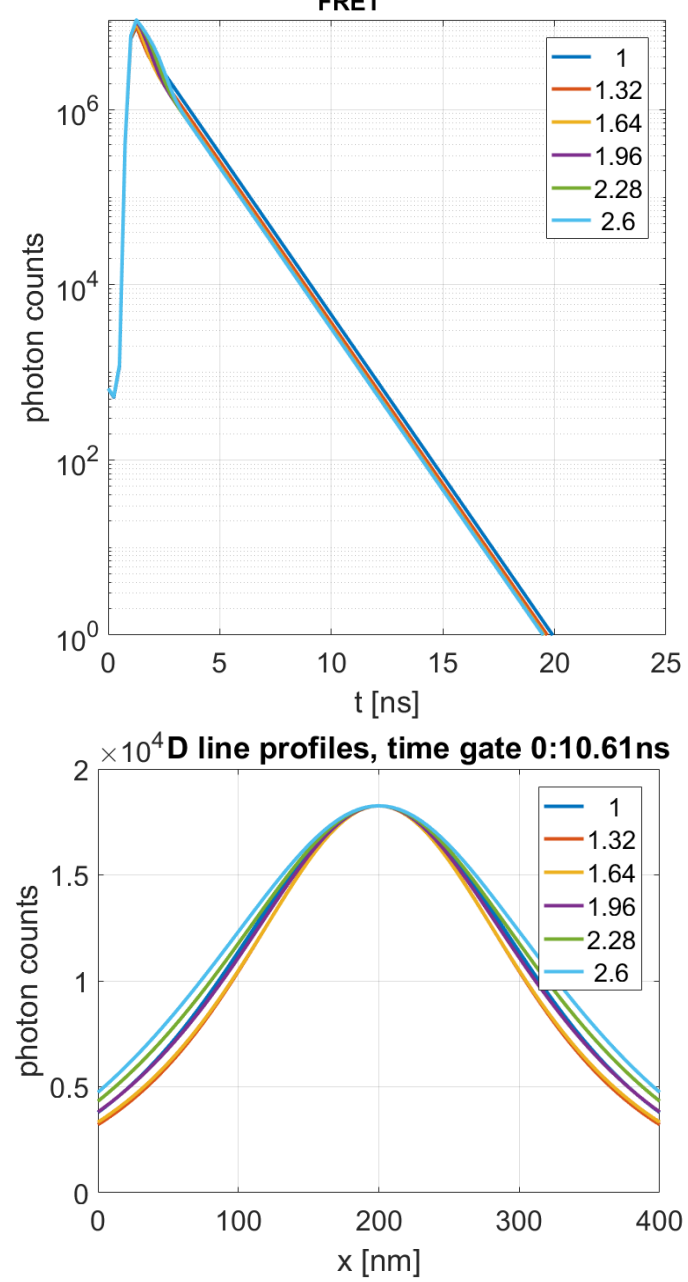

A lifetime for different STED delay, $\mathrm{E}_{\mathrm{FRET}}=\mathbf{0 . 6 9}$
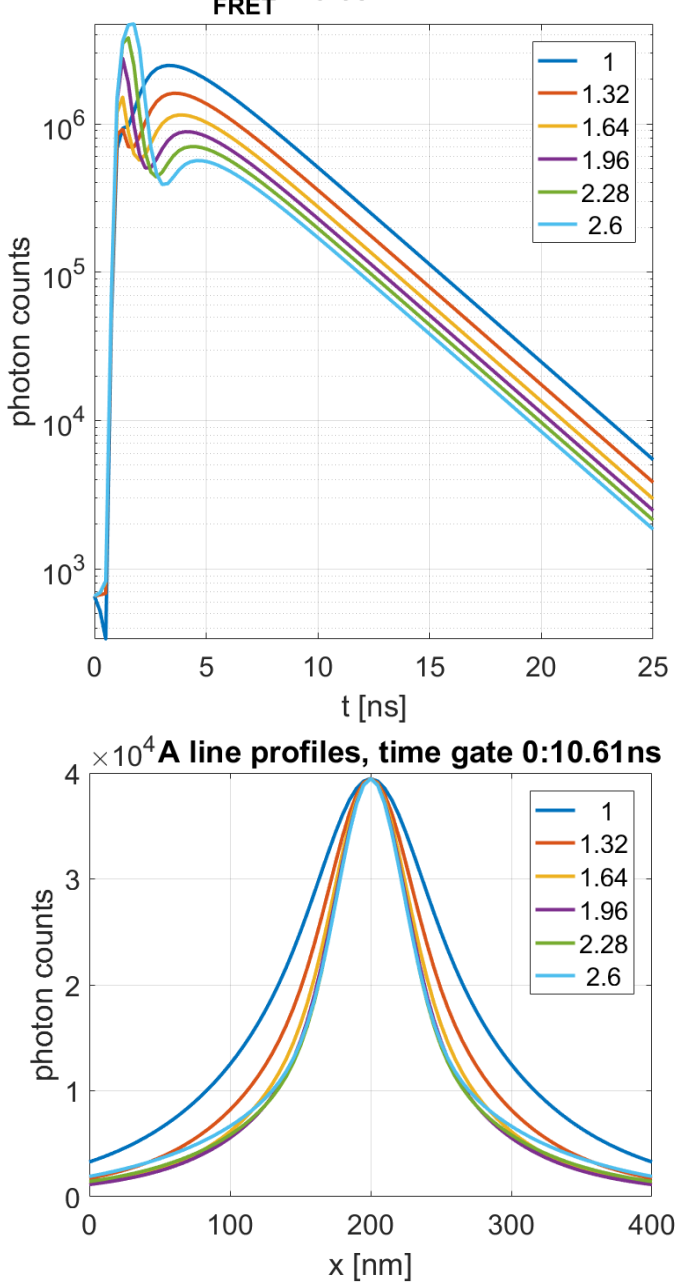

Fig. 11.3: Simulation of the excited state dynamics of a FRET pair with $69 \%$ transfer efficiency under pulsed STED light with an increasing delay between excitation and STED pulse. Top: Simulated lifetime histograms of donor and acceptor. (semi-log plots) Bottom: Simulated line profiles. The legend states the arrival time of the STED pulse in ns. The excitation pulse arrives at $\mathrm{t}=1.64 \mathrm{~ns}$.

If the STED pulse comes early, the FWHM is broader because the majority of the energy transfer occurs after depletion. If the STED pulse arrives late, many photons have been emitted before, resulting in a higher pedestal at the rim of the PSF. This is discussed in more detail in the following sections 11.5 and 11.6.

\subsection{Evolution of the acceptor's PSF over time}

In the previous section, a change of the acceptor's line profile shape due to a change of the STED pulse delay was discovered. In order to study the temporal evolution of the acceptor's spatial fluorescence intensity distribution more closely, four time gates were chosen as indicated in the lifetime histogram in Fig. 11.4: 

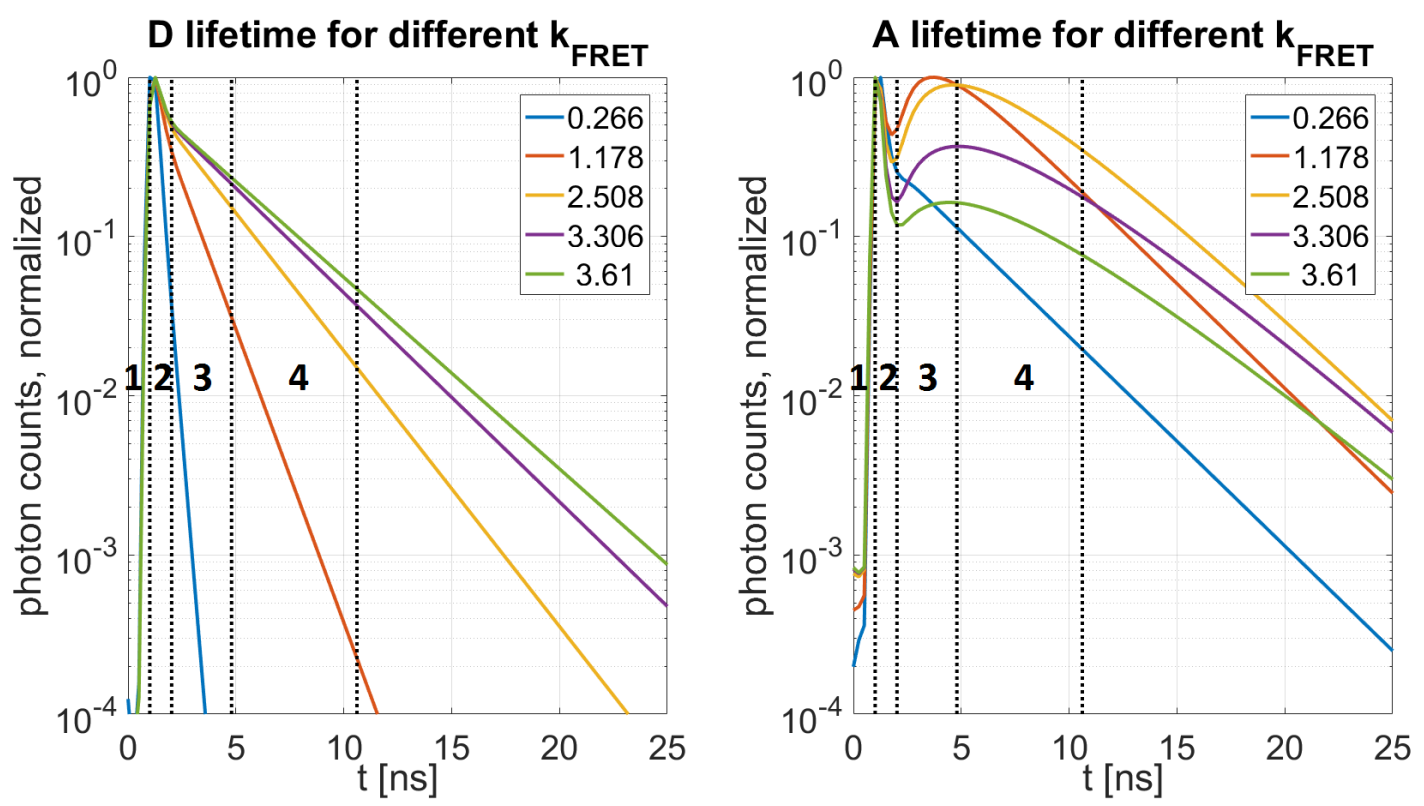

Fig. 11.4: Simulation of the excited state dynamics of FRET pairs with different transfer rates under pulsed STED light of constant intensity. The borders of the applied time gates 1 to 4 are marked with vertical black lines. The legend states the lifetime of the donor in the presence of the acceptor in ns, refer to table 10.1 .

1. before the arrival of the STED pulse

2. during the exposure to the STED pulse

3. shortly after exposure

4. long after stimulated emission depletion

The photons emitted within each time gate were used to generate a series of line profiles that is depicted in Fig. 11.5. The line profiles illustrate that the FWHM of the acceptor is narrow at first for all FRET pairs and successively becomes wider in the case of medium and low transfer rates. This is because a significant amount of energy is transferred to the acceptor after depletion and emitted later, giving rise to a higher signal at the rim of the PSF. In case of high FRET, most donors decayed within the first two time gates, whereas in case of low FRET, some excited donors are still ready to excite the acceptor within the third and fourth time gate. One can imagine the effect of FRET as a broad excitation pulse for the acceptor with the shape of the donor's lifetime histogram. In summary, a dilemma arises if the donor lifetime, and thus the FRET process, is longer than the STED pulse. Then, the depletion is imperfect, either the STED pulse arrives too early or too late.

One possibility would be to use a laser with longer STED pulses, if available, or even to use a CW-STED laser. The second approach to circumvent this dilemma is outlined in the following section 11.6, namely to use a donor with a shorter lifetime. 

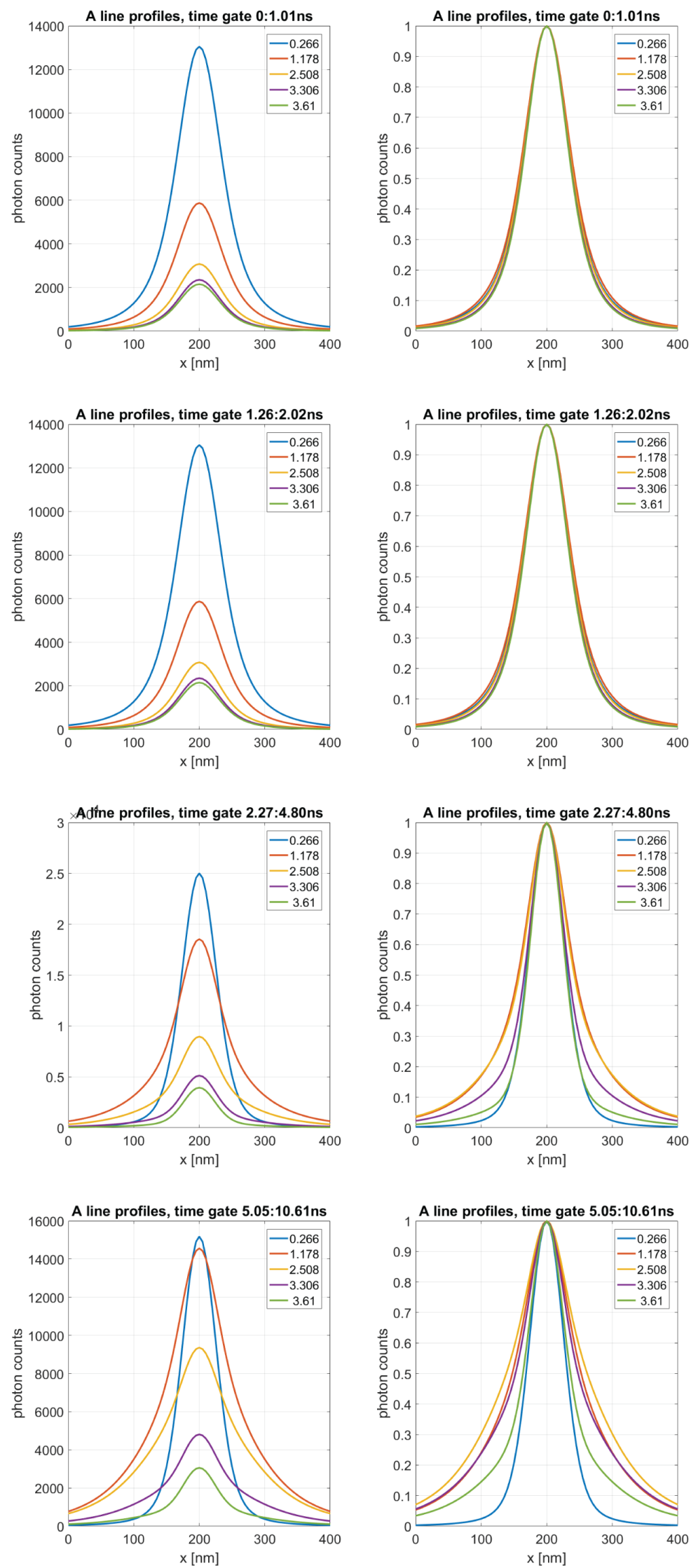

Fig. 11.5: Simulated line profiles of FRET pairs with different transfer rates under pulsed STED light of constant intensity. Only photons emitted within the time gates indicated in Fig. 11.4 contributed to the respective line profiles. Left: absolute photon counts. Right: normalized profiles. The legends state the lifetime of the donor fluorophore in ns, refer to table 10.1 . 


\begin{tabular}{|l|l|l|l|l|l|}
\hline$E_{\text {FRET }}$ & 0.20 & 0.40 & 0.50 & 0.60 & 0.80 \\
\hline$\tau_{\text {DA }}(\mathrm{ns})$ & 0.40 & 0.30 & 0.25 & 0.20 & 0.10 \\
\hline$k_{\text {FRET }}(1 / \mathrm{ns})$ & 0.50 & 1.33 & 2.00 & 3.00 & 8.00 \\
\hline
\end{tabular}

Tab. 11.1: Transfer rates and decreased lifetimes of Cy3.5 were calculated assuming certain FRET efficiencies as in section 10.1.

\subsection{Donor with a short lifetime}

The previous section described situations where the donor's lifetime is longer than the STED pulse. Furthermore, the donor was only slightly depleted. Therefore it can transfer energy to the acceptor after the acceptor has been depleted by the STED pulse to achieve diffraction unlimited resolution. Upon energy transfer, the acceptor can emit again, thus deteriorating the spatial resolution. As one possible solution, one could choose a donor with a very short lifetime, which matches the length of the STED pulses. If the energy transfer would take place during the STED pulse, then acceptors which were not depleted could emit afterwards within their lifetime, but would not be excited via FRET anymore. Thus, one would attain a diffraction unlimited image with optimal resolution in the acceptor channel.

Simulations with such a donor were performed, the fluorophore Cy3.5 was chosen as an example. It has a lifetime of $\tau_{D}=0.5 \mathrm{~ns}$, and a QY of 0.15 . For selected FRET efficiencies, the corresponding transfer rates and the decreased lifetimes were calculated as described in section 10.1 and are listed in table 11.1. The simulated lifetime histograms for different transfer rates between donor Cy3.5 and the same acceptor as before, Star635P, are depicted in Fig. 11.7. The STED pulse intensity and delay were kept constant at $0.075 \mathrm{a}$.u. and $0.64 \mathrm{~ns}$, respectively. The donor fluorescence decreases very rapidly in a monoexponential decay. In the acceptor histogram, a slight shoulder shortly after excitation due to the energy transfer is visible. The later part of the curve mostly follows a monoexponential decay, the time constant of which is the lifetime of the acceptor.

The same four time gates as in section 11.5, indicated in Fig. 11.6, were chosen for comparison. The line profiles of the fluorescence emission in each gate were calculated and are shown in Fig. 11.7. The FWHM of the pairs with different FRET efficiencies is almost the same. It hardly increases over time compared to the case shown in Fig. 11.5 .

Even though the FWHM remains narrow, there are still two potential drawbacks when choosing this approach. A shorter lifetime is usually linked to a lower QY, which will be discussed in the following subsection. Secondly, for analysis of the lifetime histograms 

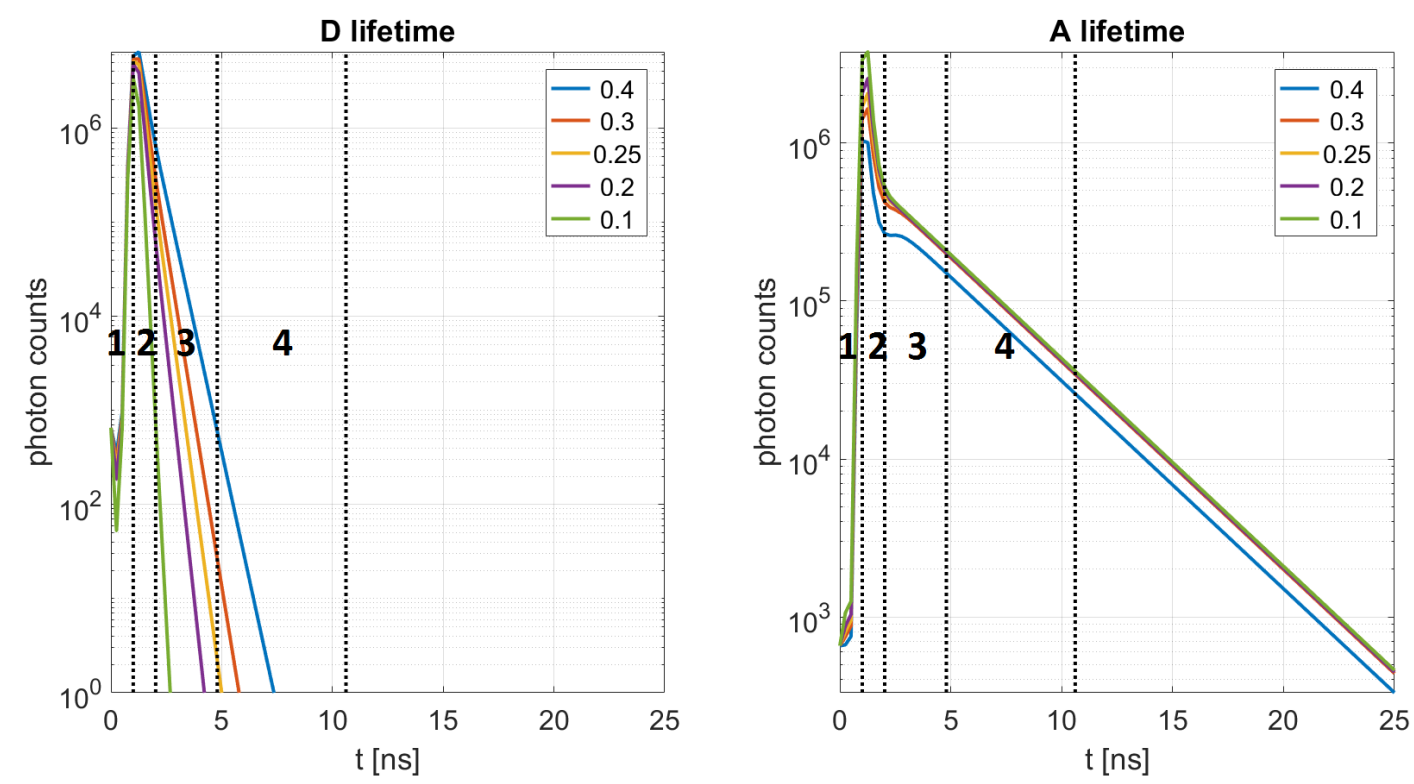

Fig. 11.6: Simulation of the excited state dynamics of FRET pairs with different transfer rates under pulsed STED light of constant intensity. The borders of the time gates 1 to 4 are marked with vertical black lines. The legend states the lifetime of the donor in the presence of the acceptor in ns, refer to table 11.1.

a short IRF is necessary to not obscure the informative first few hundred ps of the histogram which contain most of the photons.

\section{Influence of decreased donor quantum yield}

Most organic fluorophores with an optimized QY close to one have a fluorescence lifetime of approximately 3ns. Using a donor with a shorter lifetime can enable imaging with optimal spatial resolution in the acceptor channel, as described in the previous section. However, a short lifetime generally correlates with a low fluorescence QY [126]. This is because a shorter lifetime is due to a higher proportion of non-radiative decay pathways from the excited to the ground state. More details are explained in theory section 2.1. A low QY not only limits the attainable fluorescence signal, it also restricts the Förster radius $R_{0}$ :

$$
\begin{aligned}
& R_{0}^{6} \propto Q Y_{\mathrm{D}} \\
& E=\frac{R_{0}^{6}}{r^{6}+R_{0}^{6}}
\end{aligned}
$$

For example, the Förster radius of Atto532 and Star635P is 6.16nm. If the spectral overlap is constant, but the QY is decreased from 0.9 to 0.15 , the Förster radius drops to $4.57 \mathrm{~nm}$.

The MATLAB script used for the calculation of $R_{0}$ is provided in supplementary chapter 29. 

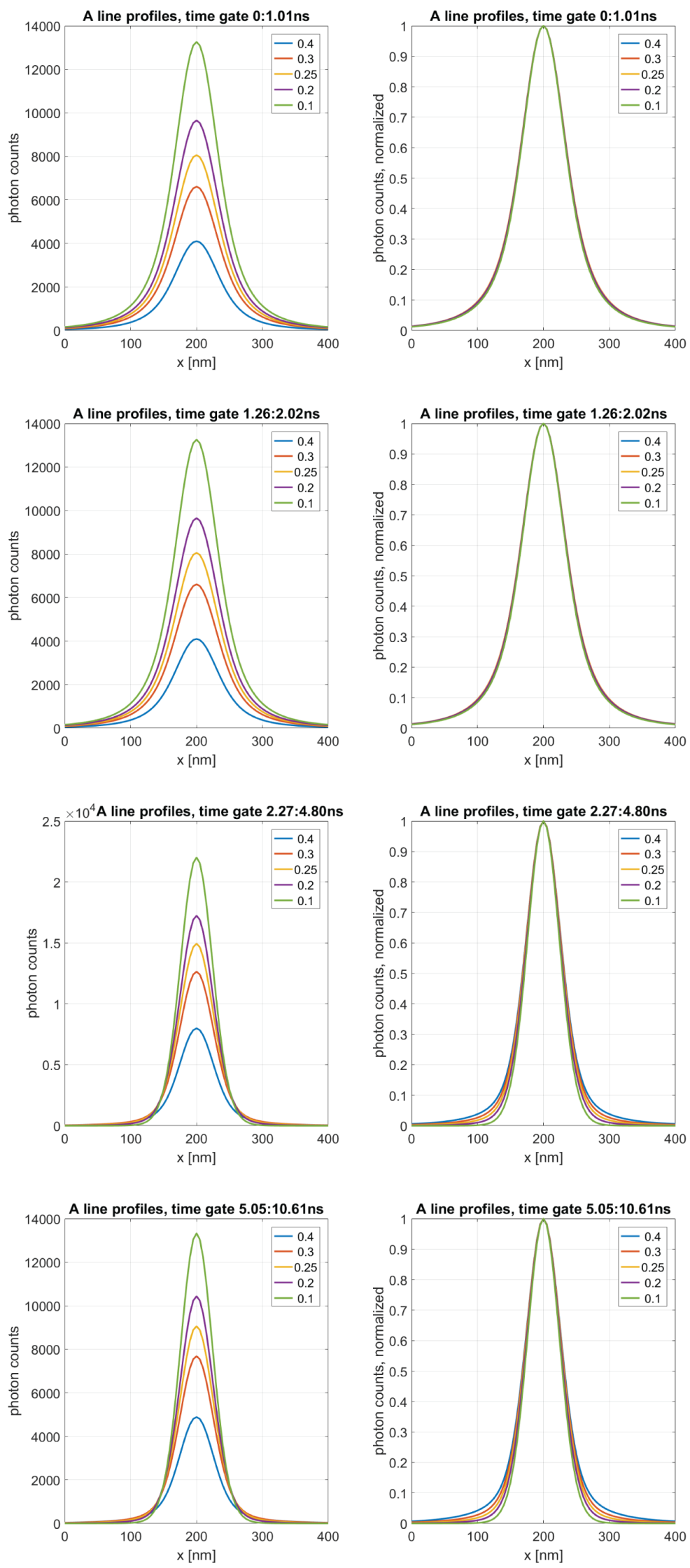

Fig. 11.7: Simulated line profiles of FRET pairs with different transfer rates under pulsed STED light of constant intensity. Only photons emitted within the stated time gates, indicated in Fig. 11.6, contributed to the respective line profiles. Left: absolute photon counts. Right: normalized profiles. The legends state the lifetime of the donor fluorophore in ns, refer to table 11.1. 



\section{Summary and discussion of the simulations}

In this part of the thesis, simulations of a single FRET pair exposed either to CW-STED or to pulsed STED light were performed. A system of rate equations described a simple model containing the ground and first excited singlet state of each molecule. Electronic transitions between both states upon absorption, spontaneous and stimulated emission and FRET were included. The rate equation system is solvable analytically only for special cases. Therefore the effect of the different parameters was studied numerically. First, the energy transfer rate was varied. Then, the STED intensity or the STED pulse delay were changed to investigate how the stimulated emission depletion interferes with the FRET process. The simulated lifetime histograms and line profiles are evaluated in view of two main objectives: the distinguishability of FRET pairs with different transfer rates, which constitutes the biological read-out of experiments, and the improvement of the spatial resolution. Regarding the latter, the acceptor's fluorescence emission is of more interest, as it can be confined efficiently by STED.

For the combination of FRET measurements with CW-STED, the influence of different FRET rates and STED intensities on the lifetime histograms was studied. Looking at the simulated lifetime histograms in Fig. 10.1 and Fig. 10.2, the main differences are the curvature within the first approximately $5 \mathrm{~ns}$ and the slope of the later part of the curve. An increase of the CW-STED intensity mainly changes the curvature, inducing a steeper decline at the beginning of the histogram. Later on, the curves run parallel, because their slope is dictated by the fluorophores' lifetimes only. A change of the FRET efficiency affects the whole curve: The speed of the transfer influences the height and width of the peak. The slope in the later part is determined by the lifetime of both dyes and is gentler if the transfer efficiency is lower. That is, if the lifetime of donor and acceptor are approximately the same. Hence, for experiments with CW-STED, a donor with a lifetime that is similar or even longer than that of the acceptor would be beneficial for discrimination of various $E_{\text {FRET }}$. It is the opposite in the pulsed STED case, where a donor with a much shorter lifetime would be advantageous. Overall, discriminating FRET pairs with different transfer efficiencies exposed to constant CWSTED intensity should be feasible if the SNR is sufficient. Whether different FRET pairs can be distinguished reliably by their lifetime histograms in images acquired with different STED intensities would need to be simulated and tested. However, such a 
test is outside the scope of this thesis. Otherwise, one would need to keep the STED intensity constant for one data set.

When looking at the lifetime histograms simulated with pulsed STED, in Fig. 11.1, Fig. 11.2 and Fig. 11.3, three main features are striking: the first peak due to excitation of the dyes and beginning energy transfer, then a sharp decrease due to depletion and after that in the acceptor's histograms a second peak or shoulder caused by energy transfer. Considering the acceptor, the height of the first peak is influenced by the transfer efficiency and its width by the delay of the STED pulse. In experiments, it will further be convoluted with the IRF. The STED intensity and pulse length determine the steepness and depth of the dip. The height and width of the second peak as well as the time point of the maximum are governed by the FRET efficiency. But additionally, a change of the STED pulse delay shifts the height and time point of the maximum. Altogether, FRET pairs with different transfer efficiencies can be distinguished by their lifetime histograms, but the STED parameters have to be included in the analysis. This will be discussed further in section 20.3.

Bearing the influence of the STED pulse delay on the histogram shape in mind, one could consider using it to better distinguish histograms of pairs with different FRET efficiencies. The ratio of the first and second peak height depends on both the transfer efficiency and the pulse delay. For example, two histograms with different known delays could be acquired and jointly analyzed in this regard. Nonetheless, one still needs to ensure optimal spatial resolution besides inference of the FRET efficiency.

A dilemma was discovered in the case of pulsed STED, namely that if the STED pulse is shorter than the donor lifetime, which dictates the duration of the energy transfer, the time-averaged spatial resolution is degraded. The deterioration of the acceptor's FWHM over time is visible when studying the line profiles in Fig. 11.1 and Fig. 11.3 and their temporal evolution in Fig. 11.5. One approach to this problem could be time gating. It could be used to exclude photons emitted before the STED pulse from image analysis to improve the resolution. Following this logic, if the lifetime of the donor is significantly shorter than that of the acceptor, the STED pulse could be delayed by that time to obtain a clean high resolution signal. An optimal pulse delay should then be found for the range of FRET rates expected in each sample. On the other hand, time gating could be used to select only photons emitted during or shortly after the STED pulse to construct a diffraction unlimited image of optimal resolution. However, for this trade-off between signal and resolution, enough fluorescence signal is necessary, otherwise a low SNR will compromise the spatial resolution again. The deterioration of the resolution could also be prevented if a donor with a lifetime comparable to the length of the STED pulse is chosen. This is illustrated by simulated line profiles in Fig. 11.7. That would be similar to the approach of time gating only photons emitted during the STED beam, only that now all the energy would be transferred during that period 
of time. Yet, as was mentioned before in section 11.6, a short fluorescence lifetime is generally coupled to a low QY. The QY in turn limits not only the intensity of the fluorescence signal, but also the Förster radius. Consequently, a low QY reduces the dynamic range for FRET measurements or vice versa a low QY entails a smaller FRET efficiency at a given inter-dye distance. On the other hand, one could use longer STED pulses if available or, going further, use a CW-STED laser.

The discussion above has shown that both combinations of FRET with CW-STED and pulsed STED have advantages and problems. High spatial resolution of FRET pairs is more easily achieved with CW-STED, implemented with pulsed excitation and time gating [128]. FRET pairs with different transfer rates are distinguishable in the simulations shown here on the basis of their lifetime histograms. How reliable this discrimination is with real data featuring low photon counts and background signal remains to be tested. In pulsed STED, optimal spatial resolution is hard to achieve, different approaches are discussed above. The discrimination of FRET pairs based on the shape of their lifetime histograms is predicted to be easier, if the STED parameters are taken into account. One practical consideration is the dependence of photobleaching on the STED pulse length, which was recently studied by Oracz et al. [91]: the longer the STED pulses, the less photobleaching occurs. The least amount of photobleaching is observed for CW-STED.

Simulated and experimentally measured lifetime histograms will be compared in chapter 18. The main features of the histograms can indeed be explained by the simple photophysical model employed in these simulations. In further experiments, photobleaching and spectral shifting need to be considered, but these belong to the domain of photochemistry.

The donor is depleted less than the acceptor, which is anticipated due to the lower absorption coefficient of the donor at the STED wavelength. From the simulated lifetime histograms, it is evident that the amount of depletion depends nonlinearly on the combination of lifetimes, FRET rates and STED pulse delays and intensity. This means that the ratio of the fluorescence intensity emitted by donor and acceptor is indeed dependent on the STED parameters. Therefore, this ratio is not a reliable measure of the FRET efficiency anymore if STED is involved. This will be discussed in detail in chapter 20. 



\section{Experimental Implementation}

This part describes the microscope setup used in this thesis (chapter 13) and the software used for measurement control and data acquisition (chapter 14). The DNA constructs bearing FRET pairs are detailed (chapter 15) and the procedures for preparing various samples are reported (chapter 16). 



\section{Microscope setup}

The optical setup is a custom built STED microscope with a hyperspectral detection unit and TCSPC capability. It is schematically visualized in Fig.13.1.

Its core with a hyperspectral detection consisting of four red detection channels was designed by Franziska Winter and Volker Westphal (MPIbpc) and built by Franziska Winter and me as described in [142]. As part of this dissertation, three detectors in the green spectrum were added to the hyperspectral detection, the TCSPC was incorporated and the control and analysis software was implemented.

In this chapter follows a description of the optical setup and of its electronic control.

\subsection{Optical setup}




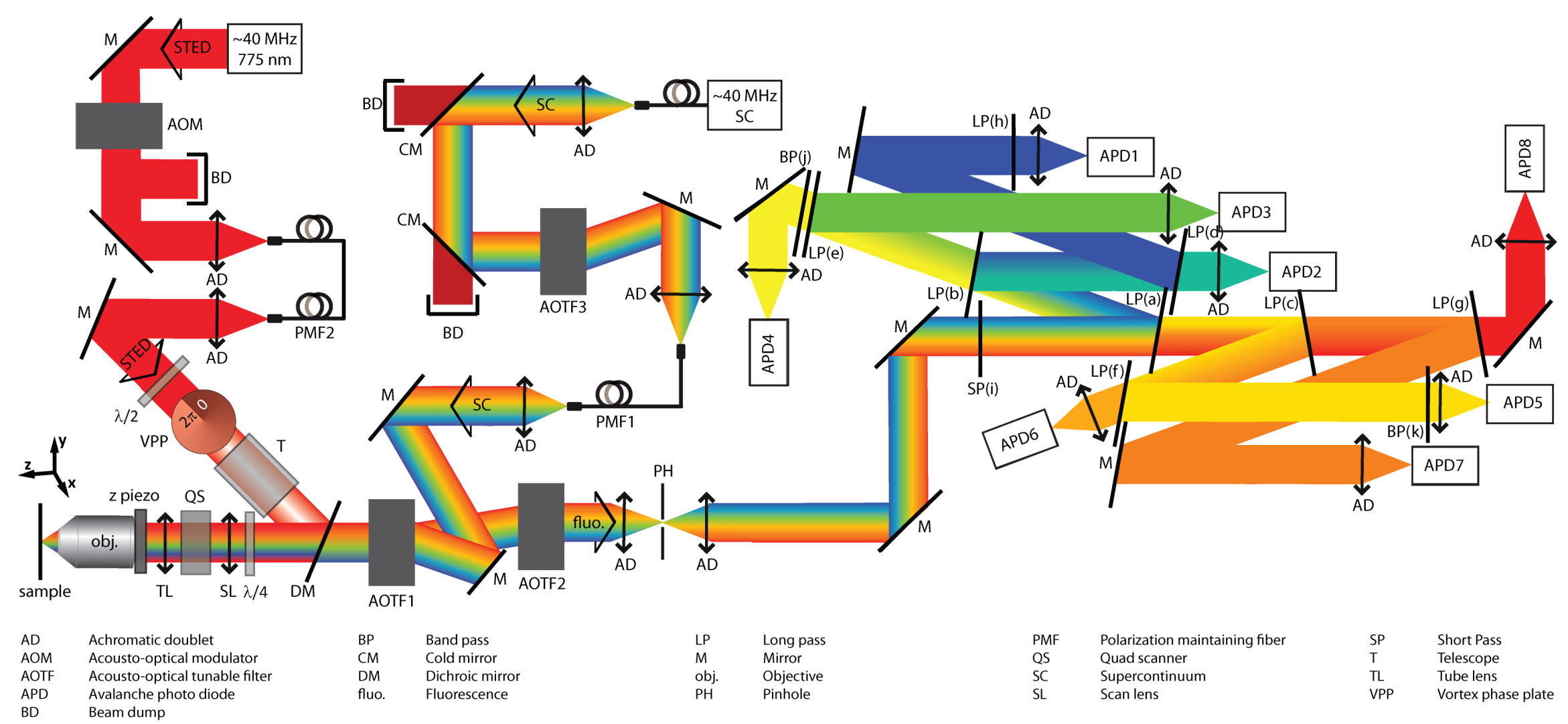

Fig. 13.1: Overview of the optical setup. The excitation light is provided by a SC laser, an arbitrary wavelength is selected by an AOBS consisting of three AOTFs. The STED laser power is modulated by an AOM; a vortex phase plate in conjunction with a quarter-wave plate creates the torus-shaped STED focus. Both beams are overlayed by a dichroic mirror and scanned with a quadscanner. The fluorescence light passes the AOBS and is filtered by a confocal pinhole before entering the hyperspectral detection unit. A cascade of longpass filters splits the fluorescence into different spectral bands, each is detected by a separate APD. Figure adapted from [142]. 


\section{Excitation beam}

The excitation light was provided by a supercontiuum (SC) pulsed laser operating at $40 \mathrm{MHz}$. Two cold mirrors removed the infrared portion of the SC emission. Filtering of the excitation wavelength from the SC was performed by an acousto-optical beam splitter (AOBS) consisting of three acousto-optical tunable filters (AOTFs). The wavelength and intensity of the diffracted beam can be chosen by modulating the radio frequency and amplitude applied to the AOTF crystal. Details about the AOTF's mode of operation are given in supplementary section 27.2. The experimental parameters which were used are listed in supplementary section 31 . AOTF3 performed a first filtering step. The polarization maintaining fiber PMF1 behind it acted like a pinhole to suppress transmitted sidebands. Behind the fiber AOTF1 further filtered the light in order to select a final excitation bandwidth of $2 \mathrm{~nm}$. For more details about the functioning and performance of the AOBS refer to chapter 3.2 of [142].

\section{STED beam}

The STED laser with a wavelength of $775 \mathrm{~nm}$ had a maximal output power of $3 \mathrm{~W}$. It was triggered by the SC laser with a variable time delay, as depicted in Fig.13.3. Regulation of the STED beam intensity was achieved with an acousto-optical modulator (AOM), whose mode of operation is outlined in supplementary section 27.1. The fiber PMF2 guided the light to another part of the optical table. A half-wave plate optimized for $600-900 \mathrm{~nm}$ in conjunction with a quarter-wave plate optimized for 600-900nm ensured a circular polarization of the beam. A vortex phase plate in the beam path imprinted a continuous helical phase change in space ranging from 0 to $2 \pi$ radians on the beam. The circular polarization and the phase change acted together to create a torus-shaped focal spot by destructive interference. A telescope with a magnification of $4 / 3$ widened the beam diameter to overfill the back aperture of the objective.

\section{Sample positioning and scanning}

A dichroic mirror, optimized for an angle of incidence of $22.5^{\circ}$, was used to superimpose excitation and STED beam. A quadscanner (QS) consisting of four galvanometer driven silver coated mirrors scanned both beams across the sample laterally with a field of view of up to $65 \mu \mathrm{m}$. One pair of mirrors deflected the beams along the first scan axis, the other pair of mirrors along the second axis. A dual power supply provided a DC voltage of 18-24V to the galvanometer controllers. For more details on the QS see [39]. The QS was attached to a side port of a commercial microscope stand. This stand afforded a widefield bright and fluorescence view of the sample and allowed manual movement of the objective for focusing. On top of the stand a stage for manual coarse lateral 


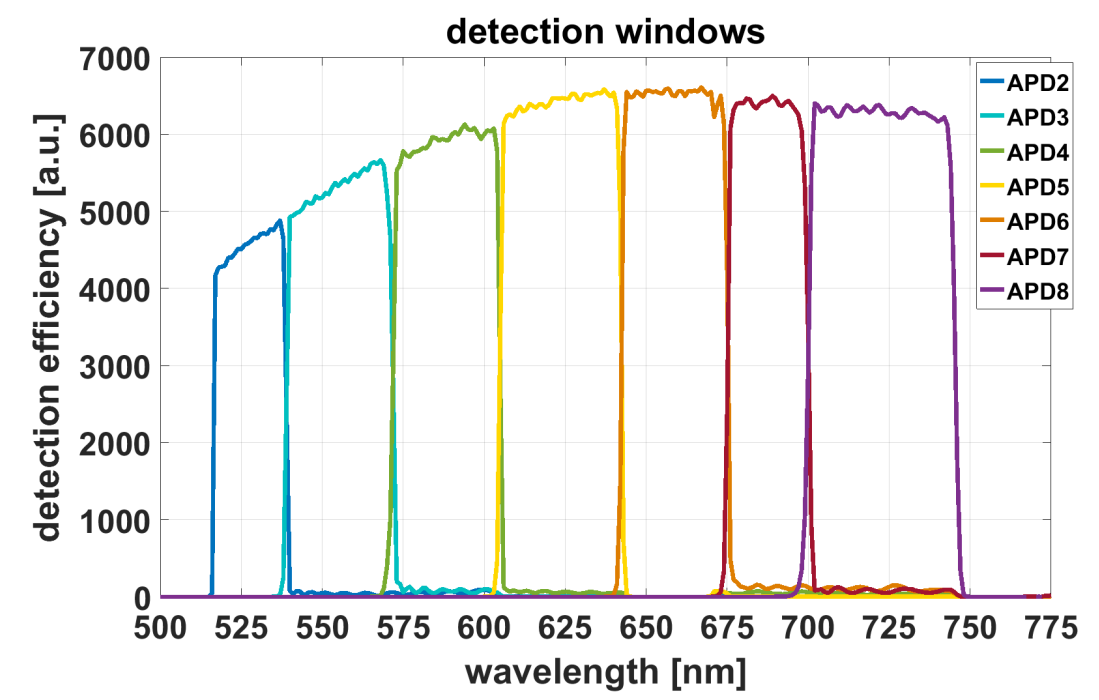

Fig. 13.2: Spectral windows of the seven APDs as defined by the edges of the longpass filters, taking the spectral detection efficiency of the APDs into account. Figure adapted from [142].

positioning of the sample was attached. For all measurements a $100 \times$ oil objective with a NA of 1.4 was used. For scanning along the optical axis, the objective was mounted on a one axis piezo stage with a travel range of $200 \mu \mathrm{m}$ in closed feedback loop control for good z stability.

\section{Fluorescence detection}

Fluorescence light emitted from the sample passed the QS, DM and AOTF1 in the zeroth order beam. No wavelength selection occured, but the fluorescence beam was slightly dispersed. AOTF2, which was arranged mirrored to AOTF1, corrected for this dispersion. The transmission of the AOBS was well above $90 \%$ for both polarization directions [142]. The fluorescence was focused through a $200 \mu \mathrm{m}$ pinhole, corresponding to $1.26 \mathrm{AU}$, to achieve confocal imaging. A shortpass filter was placed behind the pinhole to reject any STED light. Separation of the fluorescence light into different spectral channels was done by a cascade of longpass filters which guided the different wavelengths to different avalanche photodiodes (APD). The resulting spectral detection windows as defined by the edges of the longpass filters are shown in Fig. 13.2. Lenses with a focal length of $40 \mathrm{~mm}$ focussed the light onto the detection areas of the APDs. For more details on the arrangement and mounting of the longpass filters and lenses refer to chapter 3.4 of [142]. Usually, four of the detectors were used simultaneously. The APD on which scattered excitation light would fall was mostly turned off. If needed, additional bandpass filters were placed in front of APD4 and APD5 to reject 612nm excitation light. 
The cascade of longpass filters allows spectral discrimination, thus enabling the measurements of spectral shifts shown in chapter 21. But spectral discrimination comes at the expense of detection sensitivity. If four longpass filters with $90 \%$ transmission each are placed in front of the APD, only $0.9^{4}=66 \%$ of the fluorescence can pass all in all. If only FRET measurements in two detection channels would have been planned, one could have split the emission with a longpass filter into two spectral channels defined by one broad bandpass filter each. In such a design, $0.9 \cdot 0.95=86 \%$ of the fluorescence could reach the detector. Additionally, a bandpass of for example $45 \mathrm{~nm}$ width instead of $33 \mathrm{~nm}$ as in our design would admit $36 \%$ more photons to each detector, resulting in 1.8 times more signal in total. 


\section{List of components}

$\mathrm{AD}$

AOM

AOM driver

AOTF

APD

$\mathrm{BP}(\mathrm{j})$

$\mathrm{BP}(\mathrm{k})$

$\mathrm{CM}$

$\mathrm{DM}$

half-wave plate

LP(a)

$\mathrm{LP}(\mathrm{b})$

$\mathrm{LP}(\mathrm{c})$

$\mathrm{LP}(\mathrm{d})$

$\mathrm{LP}(\mathrm{e})$

LP(f)

$\mathrm{LP}(\mathrm{g})$

LP(h)

microscope stand

objective

PMF1

PMF2

piezo stage

QS mirrors

QS power supply

quarter-wave plate

SC laser

SL

SP(i)

STED laser

TL

VVP
G052012000, Qioptiq, Göttingen, DE

MTS40-A3-750.850, A-A Opto-Electronic, Orsay, FR

MODA40-B2-30, A-A Opto-Electronic, Orsay, FR

PCAOM 97-02838-01, Gooch \& Housego, Ilminster, GB

SPCM-ARQH-13, Excelitas Technologies Corp., Waltham, US

Chroma HQ580/40M

Chroma HQ650/60M

M254C45, Thorlabs, Newton, US

KP750, MSO Jena, now Optics Balzers, Balzers, LI

B. Halle Nachfl. GmbH, Berlin, DE

Semrock BLP01-594R-25

Semrock BLP01-532R-25

Semrock BLP01-664R-25

Semrock LP02-514RU-25

Semrock BLP01-561R-25

Semrock BLP01-633R-25

Semrock FF01-692/LP-25

Semrock BLP01-473R-25

DMI6000CS, Leica Microsystems, Wetzlar, DE

HCX APO CL 100x, Leica Microsystems, Wetzlar, DE

PM488, Thorlabs, Newton, US

780PM, Thorlabs, Newton, US

Mipos250 CAP, Piezosystem Jena, Jena, DE

6215HSB, Cambridge Technology, Bedford, US

3032B, Protek, Malé, MDV

B. Halle Nachfl. GmbH, Berlin, DE

EXU-6, NKT Photonics, Birkerød, DK

$\mathrm{f}=50 \mathrm{~mm}$, ARB2 coating, Qioptiq, Göttingen, DE

Semrock F75-750

Katana08-HP, Onefive, Zürich,CH

$\mathrm{f}=200 \mathrm{~mm}$

RPC Photonics, Rochester, US 


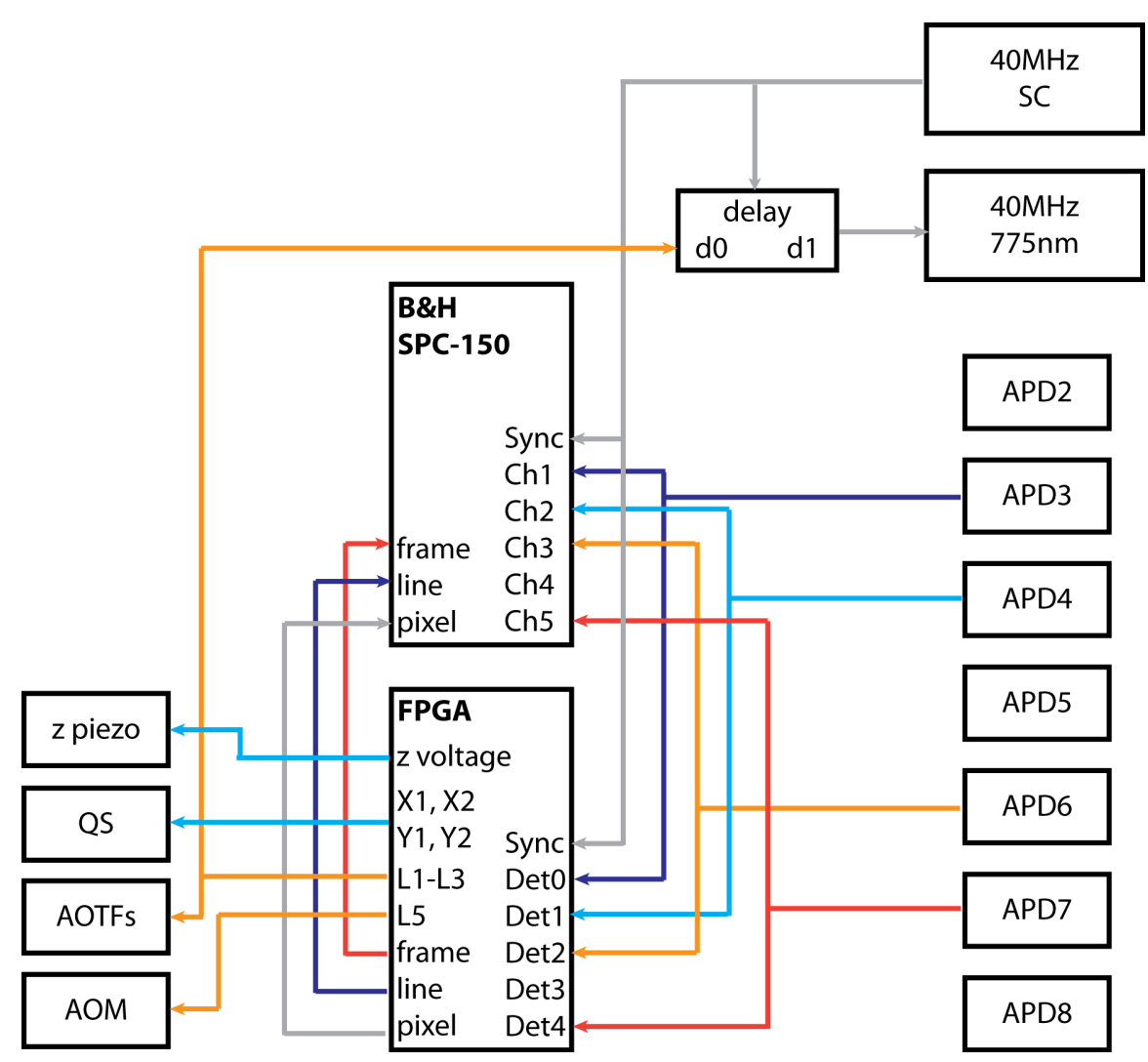

Fig. 13.3: Electronic control of the setup. Laser trigger pulses were delayed by a variable amount of time by the electronic dual channel delay unit. The FPGA board controlled both scanning and data acquisition. TCSPC was performed with the SPC-150 card from Becker\&Hickl.

\subsection{Electronic control}

\section{Laser beam control}

The SC laser provided a NIM trigger output at its pulse repetition rate which was variably delayed by an electronic dual channel delay unit before triggering the STED laser. Setting the appropriate STED pulse delay for each excitation wavelength was necessary because within each SC laser pulse different wavelength components arrived at slightly different times. One of two pre-set delay values d0 or d1, each matching one of two pre-set excitation wavelengths, could be chosen by applying low or high voltage at two inputs. For switching between the delay values, the voltage signals L1, L2 and L3 were provided by the field-programmable gate array (FPGA) board. During the first line scan, L2 was activated; during the second scan of the same line with the other wavelength, L3 was activated. L1 was always on. The same voltage signals L1 to L3 were supplied to the NI-DAQ card controlling AOTF1 and AOTF3 to switch the excitation wavelength in this line-wise fashion during scanning. The additional marker 
signal L5 was passed to the AOM to turn the STED intensity up during a line scan and down during scanner flyback to prevent excess damage of the fluorescent dyes.

\section{Scanning and Imaging}

The FPGA board generated the analog voltage signals X1, X2, Y1 and Y2 which controlled the movement of the four QS mirrors to scan the beams laterally across the sample. It also controlled the piezo stage of the objective during scanning along the optical axis.

The FPGA board accepted signals from up to four APDs simultaneously to build up intensity images during scanning. Thus four of the seven built-in detectors could be connected arbitrarily. Optionally each detection channel could be time gated to eliminate early fluorescence or background photons from the images. In order to synchronize the time gates, the trigger signal of the SC laser was passed to the FPGA board as well.

\section{TCSPC}

The SPC-150 card received the signals from four APDs as well as the SC laser trigger signal in order to perform TCSPC. Additionally, the clock signals marking pixel, line and frame steps were passed to it from the FPGA board. The information was read out by the software Imspector to build up a lifetime histogram for each pixel. The number of bins in the histogram could be freely adjusted. Additionally, the raw photon stream from the SPC-150 card could be stored for later analysis. The input Ch4 of the SPC-150 was not working properly, therefore the inputs Ch5 and Det4 were used.

\section{List of components}

$\begin{array}{ll}\text { AOM driver } & \text { MODA40-B2-30, A-A Opto-Electronic, Orsay, FR } \\ \text { AOM control } & \text { plug-in card custom built by MPIbpc IT \& Electronics Service } \\ \text { B \& H TCSPC module } & \text { SPC-150, Becker\&Hickl GmbH, Berlin, DE } \\ \text { B \& H router } & \text { HRT-82, Becker\&Hickl GmbH, Berlin, DE } \\ \text { B \& H breakout box } & \text { BOB-101, Becker\&Hickl GmbH, Berlin, DE } \\ \text { Dual channel delay } & \text { plug-in card custom built by MPIbpc IT \& Electronics Service } \\ \text { FPGA board } & \text { PCIe-7852R, National Instruments, Austin, US } \\ \text { FPGA breakout box } & \text { NI SCB-68, National Instruments, Austin, US } \\ \text { NI-DAQ card } & \text { NI PCI-6731, National Instruments, Austin, US } \\ \text { NI-DAQ breakout box } & \text { NI BNC-2110, National Instruments, Austin, US }\end{array}$




\section{Data acquisition}

\section{4}

\subsection{Measurement acquisition software}

\section{Imspector}

The software Imspector (version 0.15.12646-win32) was used for the following tasks:

- to control the QS with analog voltage signals for lateral scanning

- to control the piezo stage for scanning along the optical axis

- to read out detector signals for (time gated) intensity image acquisition

- to perform FLIM with the SPC-150 card

- to select the excitation wavelength and power via the AOTFs

- to switch the STED pulse delay

- to switch the STED laser power via the AOM

\section{Python interface}

Through Imspector's Python interface specpy (https://pypi.python.org/pypi/specpy), Python (Miniconda 3, 3.6.0) scripts were used to sequentially scan multiple small regions of interest and to save the acquired images automatically. The Python interface allows the user to set measurement parameters, to activate and start measurements and read out image stacks from Imspector. The Python scripts are given in supplementary chapter 30. They saved regions of interest (ROI) as .mat files for later analysis in MATLAB.

\section{LabView AOTF control}

The AOTFs were controlled with a custom LabView (2011, version 11.0.1f2) program which is described in detail in [80]. The radio frequency and amplitude to be applied to the AOTF's crystal, corresponding to diffracted wavelength and its intensity, were pre-set in the AOTF controller in constant USB mode. The scripts are appended in supplementary chapter 31 . Fast switching between different channels of the AOTF controller, each programmed with a different radio frequency, was done in the controller's 
frequency shift keying (FSK) mode. In FSK mode, the NI-DAQ card generated voltage signals in response to the marker voltages L1, L2 and L3. The signals were applied to the blank pin and three FSK pins of the AOTF controllers host modulation interface, such that a certain combination activated a certain channel.

\subsection{Single molecule measurement setup and workflow}

Images were acquired with Imspector in four detection channels. These were defined in the software as follows: For photolysis measurements, four APDs were connected to the FPGA board and a single excitation wavelength was used to record four images simultaneously. For FRET measurements, two APDs were connected and two excitation wavelengths were used in an alternating fashion, such that for each scan with each wavelength, two images were acquired simultaneously by both detectors.

First, a larger region of $10 \times 10 \mu \mathrm{m}$ was scanned with Imspector to provide an overview of the sample in confocal mode. This image was exported to Python, where a peak finding algorithm located single molecules. A list of the identified peak positions was used to perform multiple smaller scans with Imspector.

At each peak position, a medium-sized confocal scan of $450 \times 450 \mathrm{~nm}$ was performed. For STED measurements, the Python script located the center of intensity of this medium image (frames added) to refine the scan position. In the last step, a region of $110 \times 110 \mathrm{~nm}$ or $250 \times 250 \mathrm{~nm}$ was scanned with the STED beam turned on.

Both medium and small ROI were scanned multiple times quickly so that in the case of bleaching, an image of the complete PSF would still be formed. During both medium and small scan TCSPC was performed with the SPC-150 and a lifetime histogram for each pixel was acquired by Imspector.

Taken together, each medium and small image stack had five dimensions: scan in $\mathrm{x}$, scan in y, multiple frames and multiple bins of the lifetime histogram for each of the four detection channels. Both medium and small stacks were saved as .mat files for later analysis. The values of the different scan parameters are given in supplementary table 32.2 . 


\begin{tabular}{|l|c|c|c|c|c|c|}
\hline delay (a.u.) & 2600 & 2640 & 2700 & 2740 & 3000 & 3040 \\
\hline delay (ns) & 14.08 & 14.40 & 14.72 & 15.04 & 15.36 & 15.68 \\
\hline
\end{tabular}

Tab. 14.1: STED pulse delay

\section{STED pulse delay}

The delay of the STED pulse with respect to the electronic trigger signal was regulated by the custom dual channel delay shown in section 13.2 . Two values $\mathrm{d} 0$ and $\mathrm{d} 1$ were preset manually and toggling between both was controlled by voltage signals. Commonly used values are stated in Tab. 14.1 in units of the delay card and are converted into ns. Since these numbers refer to the delay with respect to electronic trigger pulses, they do not correspond to the value of the delay between excitation and STED laser pulses in sample. However, the chosen steps of 320ps do translate to 320ps delay changes between the laser pulses. 



\section{Fluorescently labeled DNA constructs}

\subsection{Single molecule FRET constructs}

Single molecule FRET constructs are formed by annealing two 45 base long oligonucleotides of single stranded DNA (ssDNA). One DNA strand is labeled at the 5'-end with the fluorescent dye Atto532 and at the 3'-end with biotin for immobilization via biotin-streptavidin interaction. The other DNA strand is labeled internally with the fluorescent dye Star635P via a six-carbon linker at a thymine modified with an amino group. The labeled and annealed DNA oligonucleotides were purchased from IBA GmbH (Göttingen, Germany).

In order to obtain constructs with different FRET efficiencies, the internal labeling is performed either at position 38, 33, 28, 23 or 18 of the ssDNA. The resulting distances between the fluorescent dyes are $7 \mathrm{bp}, 12 \mathrm{bp}, 17 \mathrm{bp}, 22 \mathrm{bp}$ and $27 \mathrm{bp}$. The corresponding physical distance between the fluorophores in units of $\mathrm{nm}$ was calculated with the MATLAB script given in supplementary section 29 . With this information, and the known Förster radius of $6.16 \mathrm{~nm}$, the expected FRET efficiency could be calculated and is stated in table 15.1 .

The sequences are: 5'-(Atto532)-TAA ATC TAA AGT AAC ATA AGG TAA CAT AAC GTA AGC TCA TTC GCG-3'-(Biotin) and for example 5'-CGC GAA TGA GCT TAC GT(Star635P-C6-dT) ATG TTA CCT TAT GTT ACT TTA GAT TTA-3'. The design of these FRET constructs is adapted from [72].

\begin{tabular}{|c|c|c|c|}
\hline name & $\mathbf{r}(\mathbf{b p})$ & $\mathbf{r}(\mathbf{n m})$ & EFRET $_{\text {FR }}$ \\
\hline DNA 79 & 7 & 4.0 & 0.93 \\
\hline DNA 80 & 12 & 5.4 & 0.69 \\
\hline DNA 81 & 17 & 6.9 & 0.34 \\
\hline DNA 82 & 22 & 8.5 & 0.13 \\
\hline DNA 83 & 27 & 10.1 & 0.05 \\
\hline
\end{tabular}

Tab. 15.1: Expected FRET efficiency $E_{\text {FRET }}$ corresponding to different distances $\mathrm{r}$ of fluorophores on DNA. 


\subsection{Constructs with single fluorophores}

The donor control construct consists of the DNA strand 5'-(Atto532)-TAA ATC TAA AGT AAC ATA AGG TAA CAT AAC GTA AGC TCA TTC GCG-3'-(Biotin) and the complementary unmodified ssDNA.

The acceptor control construct consists of the DNA strands 5'-TAA ATC TAA AGT AAC ATA AGG TAA CAT AAC GTA AGC TCA TTC GCG-3'-(Biotin) and 5'-CGC GAA TGA GCT TAC GT(Star635P-C6-dT) ATG TTA CCT TAT GTT ACT TTA GAT TTA-3'. 


\section{Sample Preparation}

\section{6}

\subsection{Buffers}

\subsubsection{ROXS buffer}

ROXS buffer is prepared in a similar manner as described in [130] and [55].

The basic components are put together as follows: Glucose buffer is made by dissolving $1.5 \mathrm{~g}$ glucose (Merck, 1.08342) in $15 \mathrm{ml}$ phosphate buffered saline (PBS, $137 \mathrm{mM} \mathrm{NaCl}$, $\left.2.68 \mathrm{mM} \mathrm{KCl}, 10 \mathrm{mM} \mathrm{Na}_{2} \mathrm{HPO}_{4}, \mathrm{pH} 7.4\right) .50 \mathrm{mg}( \pm)$-6-Hydroxy-2,5,7,8-tetramethylchromane2-carboxylic acid (Trolox, Fluka, 56510) are dissolved in $1 \mathrm{ml}$ acetaldehyde (Fluka, 00070) to yield $0.2 \mathrm{~mol} / 1$ Trolox solution. Glock solution is prepared by dissolving 25mg glucose oxidase (glucose oxidase type VII from aspergillus niger, Sigma-Aldrich, G2133-10KU) in $340 \mu \mathrm{l}$ PBS and adding $160 \mu \mathrm{l}$ catalase (catalase from bovine liver, Sigma-Aldrich, C100-50MG) solution. MV solution is obtained by dissolving $31.16 \mathrm{mg}$ methyl viologen dichloride hydrate (MV, Sigma-Aldrich, 85,617-7) in 1ml PBS. TCEP solution, short for Tris(2-carboxyethyl)phosphine hydrochloride (Pierce, 77712), is prepared by dissolving $2.87 \mathrm{mg}$ TCEP in $1 \mathrm{ml}$ PBS.

To get the final ROXS + ME buffer, $16 \mu \mathrm{l}$ of each component, Trolox, Glock, MV and TCEP solution, as well as $16 \mu \mathrm{l} \beta$-mercaptoethanol (ME, Sigma-Aldrich, 63689) are mixed with $1520 \mu$ l glucose buffer.

\subsubsection{DNA storage buffer}

DNA storage buffer consists of an aquaeous solution of 50mM NaCl (Sigma, S5150-1L) and 10mM Tris- $\mathrm{HCl}$ (Fluka, 93316) at $\mathrm{pH}$ 8.0.

\subsection{Dye solutions}

Freeze-dried dye is solubilized in circa $10 \mu \mathrm{l}$ dimethyl sulfoxide (DMSO, Merck, 1.02952), then diluted to a final concentration of $0.5 \mu \mathrm{M}$ using thiodiethanol (TDE, Sigma, 88559- 
250ML) for Star635P or PBS for Atto532. TDE is quite viscous, preventing the quick formation of a dye film on the coverslip surface. Rose Bengal was diluted in a $5.66 \mathrm{~mol} / 1$ potassium iodide (Aldrich, 22,194-5) solution, according to [117].

$80 \mu \mathrm{l}$ of dye solution are pipetted into a single concave microscope slide and carefully covered with a coverslip, avoiding air bubble formation. The sample is sealed on the sides using nail polish.

\subsection{Gold bead alignment samples}

A colloid of gold beads of 150nm diameter (BB Solutions, EMGC150) is diluted to a suitable degree and sonicated for $10 \mathrm{~min}$ to break up clusters. In the meantime, $75 \mu \mathrm{l}$ poly-L-lysine solution (Sigma, P8920-100ML) is spread on a coverslip. After 5min, the coverslip is rinsed first with deionized, then with distilled water and dried with pressurized air. Afterwards, $70 \mu \mathrm{l}$ of the gold bead solution are pipetted onto the prepared coverslip and left to adsorb for 2 to $5 \mathrm{~min}$. Then, the coverslip is rinsed again. Lastly, the coverslip is mounted on a microscope slide with Mowiol with DABCO (25\% (w/v) glycerol, 9\% (w/v) Mowiol 4-88, 0.1M Tris/Cl, 0.1\% (w/v) 1,4-Diazabicyclo[2.2.2]octane, $\mathrm{pH} 8.5$ ) and sealed with nail polish.

\subsection{Immunostaining of fixed cells}

Immunostaining of fixed cells for testing fluorescent dyes was always performed by Ellen Rothermel (MPIbpc) according to the following protocol. Vero cells grown on coverslips are fixed with

- 4\% paraformaldehyde (PFA) in PBS for $10 \mathrm{~min}$ at room temperature for staining the nuclear pore complex protein Nup153,

- $8 \%$ PFA in PBS for $5 \mathrm{~min}$ at $37^{\circ} \mathrm{C}$ for staining mitochondrial membrane protein Tom20, or

- $-20^{\circ} \mathrm{C}$ cold methanol for staining tubulin.

Next, the cells are permeabilized with

- $0.1 \%$ Triton X-100 (T8787, Sigma-Aldrich) in PBS for 5min for staining Nup153,

- $0.5 \%$ (v/v) Triton in PBS for $5 \mathrm{~min}$ for staining Tom20, or

- not permeabilized after methanol fixation for staining tubulin. 
Afterwards, the sample is blocked with 1\% BSA solution in PBS three times for $5 \mathrm{~min}$ each. Then, the sample is incubated with primary antibodies against the target structure for $1 \mathrm{~h}$ at room temperature. The following primary antibodies were used:

- Anti-Nup153 (QE5), Abcam ab24700

- Anti-Tom20 (FL-145), Santa Crúz Biotechnology sc11415

- Anti- $\alpha$-Tubulin (mouse), Sigma T6074

Next, the cells are washed and blocked again three times for 5min each with 1\% BSA in PBS. Then, the cells are incubated with appropriate secondary antibodies for $1 \mathrm{~h}$. Either commercial dye-labeled antibodies were used if available or the conjugation of dye and antibody was performed by Ellen Rothermel. Afterwards, the sample was again washed three times with $1 \%$ BSA in PBS for $5 \mathrm{~min}$. Finally, the sample is mounted on the microscope slide in Mowiol with DABCO.

\subsection{Cleaning of coverslips and microscope slides}

In order to reduce background from unwanted fluorescent particles on the coverslips, all coverslips are cleaned prior to sample preparation. Cleaning is performed with a $2 \%$ dilution of Hellmanex®III (Hellma GmbH \& Co. KG, Müllheim, Germany) in destilled water. The coverslips are sonicated inside the cleaning solution twice for $15 \mathrm{~min}$. After the first $15 \mathrm{~min}$, they are rinsed with deionized water and the cleaning solution is replaced. After the second 15min sonication step, the coverslips are rinsed with deionized water and dried with compressed $\mathrm{N}_{2}$.

Microscope slides are cleaned in the same manner but with only a single $15 \mathrm{~min}$ sonication step.

\subsection{Immobilization of labeled DNA}

A flow channel is self-assembled by glueing a cleaned coverslip to a cleaned microscope slide with double sided tape (Scotch $\AA$, 3M France).

The channel is filled with $20 \mu$ l biotinylated BSA (Albumin, biotin labeled bovine, SigmaAldrich, A854910MG) dissolved in PBS, $2 \mathrm{mg} / \mathrm{ml}$. After an incubation time of $10 \mathrm{~min}$ the channel is flushed with $600 \mu \mathrm{l}$ PBS. The PBS in the flow channel is replaced by $20 \mu \mathrm{l}$ Streptavidin (Streptavidin, recombinant, Sigma-Aldrich, 11721666001) dissolved in PBS, $0.5 \mathrm{mg} / \mathrm{ml}$. After an incubation time of $10 \mathrm{~min}$ the channel is flushed with $600 \mu \mathrm{l}$ PBS. The PBS in the channel is replaced by $20 \mu$ l DNA solution (200pM in PBS). After 


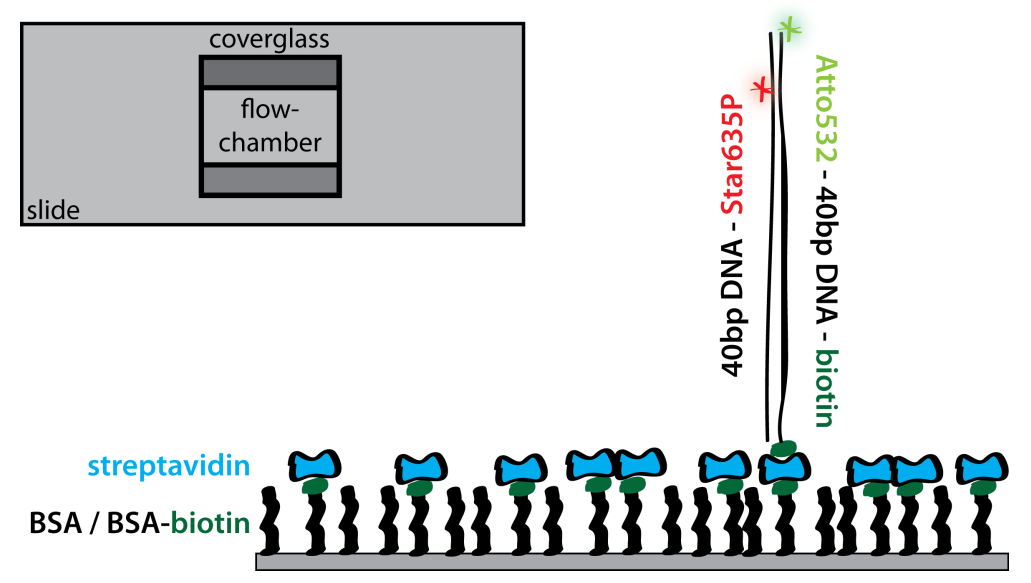

Fig. 16.1: Sketch of the fluorescently labeled DNA immobilized in the flow channel on the surface of the coverslip by biotin-streptavidin interaction.

an incubation time of 10min the channel is flushed with $600 \mu \mathrm{l}$ ROXS + ME for FRET experiments. For photoconversion experiments with single fluorophore constructs, the channel is flushed with PBS. The flow channel is immediately sealed with epoxy glue (Hysol®, Locktite). 


\section{Experimental Results}

In this thesis the interplay of FRET and STED photophysics was studied with the objective of acquiring diffraction unlimited STED images of FRET pairs from which the FRET efficiency could be deduced.

First, an efficient FRET pair of organic fluorophores which is compatible with STED had to be found (chapter 17). In order to avoid ensemble averaging in the analysis, single dye pairs immobilized on DNA strands in aqueous solution were chosen as a sample.

Fluorescence decay curves of single FRET pairs were acquired to visualize the combined effect of FRET and STED. These are compared with the simulations (chapter 18) to understand the underlying mechanisms. Moreover, high resolution images of single molecules and FRET pairs were recorded to measure the FWHM of the intensity distributions as a proxy for the achievable spatial resolution (chapter 19).

During the calculation of the spectral FRET efficiency from intensity ratios in different detection channels, it was noted that the intensity ratio depends on the STED intensity and pulse delay. Therefore, a new measure for the FRET efficiency had to be found which is applicable to STED images (chapter 20).

While analyzing the intensity ratios in different detection channels, spectral shifting of the fluorophores was observed (chapter 21). Implications for STED imaging and FRET measurements (chapter 22) were investigated. 



\section{Selection of a STED-compatible dye pair for FRET experiments}

\subsection{Donor fluorophores}

A screen of FRET literature showed that donors in the green spectral region are usually paired with acceptors in the red spectral region in order to avoid excessive bleedthrough of donor emission into the FRET channel and strong direct excitation of the acceptor. Established FRET pairs with STED-compatible fluorophores are:

- Atto550 \& Atto647N

- Atto532 \& Star635P

- Atto532 \& CF680R

- Atto532 \& Atto647N

- Cy3 \& malachite green

Green fluorophores available in the department, listed in table 17.1, were tested for their brightness, photostability under 775nm STED illumination and excitability by STED light. For testing, cultured Vero cells were immunostained with dye-labeled antibodies, the protocol is provided in section 16.4. The same ROI was imaged for several frames to record photobleaching. It was scanned line-interleaved with green and red excitation and only STED light to measure the fluorescence induced by the STED beam. The image acquisition parameters are detailed in supplementary table 32.1. Mostly, the vortex phase plate was removed from the STED beam path before imaging, so that the STED light focus had a Gaussian intensity distribution. This way, the full impact of the STED beam could be recorded. The same approach was chosen in [91]. Only the measurements of Atto532 and Atto550 on Aug. 13th 2015 were performed with a doughnut-shaped STED focus. In Fig. 17.1, one can see that the excitation by the STED beam seems to be much lower for these measurements. This has two reasons: First, fluorescence originating from the area of the doughnut ring is mostly blocked by the confocal pinhole. Second, the peak intensity of a doughnut-shaped focus is approximately three times lower than that of a Gaussian focus at equal beam power because the light is spread over a larger area. 


\begin{tabular}{|l|c|c|c|c|}
\hline dye & $\lambda_{\text {abs }}(\mathbf{n m})$ & $\lambda_{\text {em }}(\mathbf{n m})$ & $\mathbf{Q Y}$ & $\tau(\mathbf{n s})$ \\
\hline Atto514 & 511 & 532 & 0.85 & 3.9 \\
\hline Atto520 & 517 & 538 & 0.90 & 3.6 \\
\hline Atto532 & 532 & 552 & 0.90 & 3.8 \\
\hline Atto542 & 542 & 562 & 0.93 & 3.7 \\
\hline Atto550 & 554 & 576 & 0.80 & 3.6 \\
\hline Atto565 & 564 & 590 & 0.90 & 4 \\
\hline Alexa514 & 518 & 540 & - & - \\
\hline Alexa532 & 532 & 553 & 0.61 & 2.5 \\
\hline Alexa546 & 556 & 573 & 0.79 & 4.1 \\
\hline Alexa555 & 555 & 565 & 0.10 & 0.3 \\
\hline Star512 & 511 & 530 & 0.82 & 4.1 \\
\hline DyL485LS & 485 & 559 & - & - \\
\hline Dy485XL & 485 & 560 & - & - \\
\hline
\end{tabular}

Tab. 17.1: Absorption and emission maxima, QY and fluorescence lifetime of tested green fluorophores according to the manufacturer.

Fluorophores with a large Stokes shift were also tested. Because of their blue absorption spectrum, direct excitation of the red acceptor dye could mostly be avoided. However, the chosen long-Stokes dyes could not be excited sufficiently below approximately $500 \mathrm{~nm}$ with the available setup.

In order to assess the bleaching rate, the photon counts in every image frame were summed and then all values were normalized to the brightest frame. The corresponding MATLAB code is provided in supplementary section 29. Three measurements were performed in different sample regions with the same STED intensity and the fluorescence signals were averaged. The resulting decay curve of intensity over frames was fit with a single exponential decay in the software Origin. The decay rate was converted to $1 / \mathrm{ns}$ by dividing by the duration of one frame scan. Measurements for a range of STED intensities were done to estimate the dependence of the bleaching rate on the intensity. The results are plotted in the left half of Fig. 17.1, the error bars correspond to the error of the exponential decay fit.

Next, the magnitude of re-excitation of the fluorophores by the STED beam itself was evaluated. To do so, the summed photon count per frame upon excitation by $775 \mathrm{~nm}$ of varying intensity was divided by the photon count excited by green light of constant intensity. The MATLAB code is appended in supplementary section 29. Again, measurements were performed for a range of STED intensities with three measurements each, which were averaged and the standard deviation plotted as error bars. The resulting excitation ratios are shown in the right half of Fig. 17.1. The approximately quadratic dependency indicates that the fluorophores are mostly excited by two-photon absorption. 

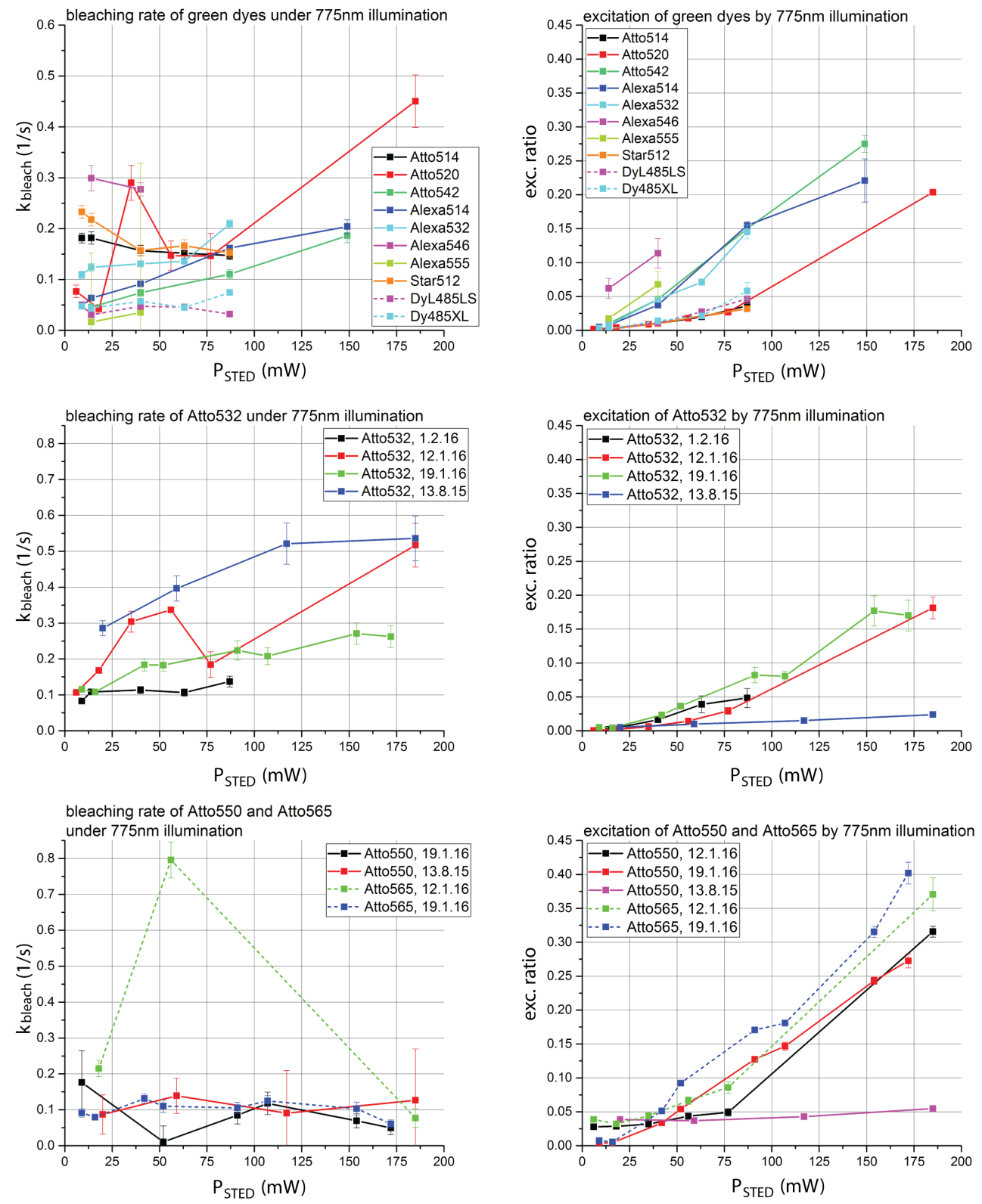

Fig. 17.1: Left: Bleaching rates $k_{\text {bleach }}$ of tested green fluorophores under 775nm STED illumination of increasing intensity. Error bars correspond to the error of the single exponential decay fit. Right: Excitation of the green fluorophores by the STED beam whose intensity was increased step by step. The excitation ratio is the ratio of the emission due to the STED beam and the emission due to blue excitation light of constant power. Error bars correspond to the standard deviation of three measurements at the same power. The vortex phase plate was removed from the STED beam for all measurements except those of Atto532 and Atto550 performed on 13th Aug. 2015. 


\begin{tabular}{|l|c|c|c|c|}
\hline dye & $\lambda_{\text {abs }}(\mathbf{n m})$ & $\lambda_{\text {em }}(\mathbf{n m})$ & $\mathbf{Q Y}$ & $\tau(\mathbf{n s})$ \\
\hline DyL633 & 638 & 658 & 0.06 & - \\
\hline Star635P & 638 & 651 & 0.90 & 3.3 \\
\hline StarRed & 638 & 655 & 0.90 & 3.4 \\
\hline Atto633 & 630 & 651 & 0.64 & 3.3 \\
\hline
\end{tabular}

Tab. 17.2: Absorption and emission maxima, QY and fluorescence lifetime of tested red fluorophores according to the manufacturer. The QY of DyL633 was taken from [90].

Atto532 was chosen as donor for further experiments. It was one of the brightest fluorophores. Its bleaching rate is higher than that of Atto550 and Atto565, but comparable to that of the other tested fluorophores. Atto550 and Atto565 were dismissed because they showed significant spectral bleedthrough into the acceptor detection channel and because their optimal excitation wavelength also directly excited the acceptor fluorophores efficiently.

\subsection{Acceptor fluorophores}

During my Diploma thesis work, Franziska Winter and I tested the performance of red fluorophores under $775 \mathrm{~nm}$ STED. The resulting list of suitable fluorophores can be found on page 70 of [142]. For four of these fluorophores, listed in table 17.2, bleaching rates and excitation by the STED beam were quantified. This was done in the same way as described for the green fluorophores in the previous section, by imaging fixed cells labeled with dye-conjugated antibodies over several frames with red excitation light. The scanning parameters are detailed in supplementary table 32.1. Contrary to the green fluorophore measurements, the red fluorophores were imaged at that time with a doughnut-shaped focus. The results are stated in tables 17.3 and 17.4.

The bleaching rates $k_{\text {bleach }}$ of the red fluorophores are much lower than those of the green fluorophores, even though the red fluorophores have a higher absorption coefficient at $775 \mathrm{~nm}$. This is because the red fluorophores are more efficiently transferred back to the ground state by the STED beam and do not remain in the excited state for a long time. Bleaching reactions usually occur from higher excited states or triplet states, which are reached via the excited state.

The bleaching rates were extracted from images acquired with red excitation. Upon green or yellow excitation, an initial increase in fluorescence or a decrease not following a single exponential decay were often observed. This is attributed to blue-shifting of the emission after photoconversion of the fluorophores. More details are given in chapter 21. Exemplary curves of slight blue-shifting of the fluorescence emission in ensemble 


\begin{tabular}{|l|c|c|c|c|}
\hline dye & $\begin{array}{c}\mathbf{P}_{\text {STED }}=\mathbf{1 1 4 m W} \\
\mathbf{k}_{\text {bleach }}(\mathbf{1} / \mathbf{s})\end{array}$ & $\begin{array}{c}\mathbf{P}_{\text {STED }}=\mathbf{1 1 4 m W} \\
\mathbf{\text { s.d.k }}(\mathbf{1} / \mathbf{s})\end{array}$ & $\begin{array}{c}\mathbf{P}_{\text {STED }}=\mathbf{2 4 2 m W} \\
\mathbf{k}_{\text {bleach }}(\mathbf{1} / \mathbf{s})\end{array}$ & $\begin{array}{c}\mathbf{P}_{\text {STED }}=\mathbf{2 4 2 m W} \\
\mathbf{s . d} \cdot \mathbf{k} \mathbf{( 1 / s})\end{array}$ \\
\hline DyL633 & 0.073 & 0.003 & 0.228 & 0.010 \\
\hline Star635P & 0.029 & 0.004 & 0.075 & 0.006 \\
\hline StarRed & 0.020 & 0.002 & 0.054 & 0.004 \\
\hline Atto633 & 0.027 & 0.003 & 0.182 & 0.011 \\
\hline
\end{tabular}

Tab. 17.3: Bleaching rate $k_{\text {bleach }}$ of tested red fluorophores under 775nm STED illumination at two intensities. Standard error of the single exponential fit is given.

\begin{tabular}{|c|c|c|}
\hline dye & $\begin{array}{c}P_{\text {STED }}=114 \mathrm{~mW} \\
\text { ratio exc }\end{array}$ & $\begin{array}{c}\text { P }_{\text {STED }}=242 \mathrm{~mW} \\
\text { ratioexc }\end{array}$ \\
\hline DyL633 & 0.02 & 0.06 \\
\hline Star635P & 0.04 & 0.10 \\
\hline StarRed & 0.04 & 0.09 \\
\hline Atto633 & 0.03 & 0.09 \\
\hline
\end{tabular}

Tab. 17.4: Excitation of tested red fluorophores by the 775nm STED beam at two intensities, relative to excitation with $612 \mathrm{~nm}$ at constant power.

measurements of the red fluorophores DyL633, Atto633, Star635P, StarRed, Atto594, Star600 and Alexa633 are shown in Appendix E of [142].

Bleaching rates measured at lower STED intensity could not be fitted reliably, because often a slight increase of fluorescence was observed in the first frames before it decreased in later frames. An example is shown in supplementary Fig. 28.1. Similar behaviour of red fluorophores, e.g. Atto647N, has been observed before [69] and is attributed to non-fluorescent dye aggregates which are destroyed by bleaching.

The fraction of fluorescence due to excitation by the STED beam, stated in table 17.4, is negligible. The same was reported by [91].

For further experiments, Star635P was chosen as acceptor due to its high brightness, low bleaching rate and negligible excitation by the STED beam. 


\subsection{Summary and discussion}

Green and red organic fluorophores were tested regarding their brightness, photostability and excitability by the STED beam. For further experiments, Atto532 and Star635P were chosen as FRET pair.

In general, green fluorophores bleach faster than red fluorophores under 775nm STED illumination. As they are depleted less efficiently but excited more strongly by STED light via two-photon absorption and thus spend more time in the excited state, the probability for further excitation, ISC and finally bleaching is higher. In contrast, the fraction of fluorescence emitted by the red fluorophores due to excitation by the STED beam increased linearly with the STED intensity [91], and remained negligible.

Fluorophores with a long Stokes shift were also tested as donors. Due to their blue excitation spectrum, direct excitation of the acceptor could have been minimized while maintaining a high spectral overlap of the donor's emission with the acceptor's absorption spectrum. Thus, less correction of the raw images in the FRET channel would have been necessary. Moreover, long-Stokes-shift donors have low bleaching rates under 775nm STED and are excited comparatively little by the STED beam. Unfortunately, the studied Long-Stokes fluorophores could not be excited efficiently with the supercontinuum laser source, whose emission drops off in the blue spectral range. The installation of an additional excitation laser with higher intensity at 488nm was not possible, because the custom-made dichroic mirror, which superimposes excitation and STED beam, reflects light below 500nm.

Fluorescent proteins are often used in FRET experiments. Yet, in the red spectral region, they are still inferior to organic fluorophores regarding their brightness and photostability in STED experiments. Therefore, only organic dyes were studied here. They can also be targeted to proteins of interest via genetically encoded SNAP-, CLIP- or HALO-tags. 


\section{Comparison of measured and simulated decay curves}

In chapter 11 , the simulation of fluorescence decay curves ${ }^{1}$ with a system of rate equations based on a simple photophysical model was described. The model included electronic transitions between ground and first excited singlet state upon photon absorption, spontaneous and stimulated emission and FRET. In this chapter, the simulated decay curves are compared to measurements in order to check if that simple model is sufficiently accurate, or if additional electronic states and transitions are required.

The fluorescence emitted by single FRET pairs of Atto532 and Star635P immobilized on a coverslip surface as detailed in section 16.6 was acquired in two different spectral channels with TCSPC as described in section 14.2. The fluorescence decay curves collected from over 100 FRET pairs in the same sample under constant illumination conditions were added to improve the SNR. A single fluorescence decay curve can be seen in section 22.2. All added decays were normalized and deconvolved with the corresponding IRF of that detection channel. The IRF was obtained by recording the fluorescence of Rose Bengal in a $5.66 \mathrm{~mol} / 1$ potassium iodide solution, which has a fluorescence lifetime of only 16ps [117].

The resulting deconvolved fluorescence decay curves of different FRET pairs imaged with various STED parameters are plotted in semi-log graphs in Fig. 18.1. On the left, decays acquired in the donor channel, meaning the green detector with green excitation, are shown, and on the right, decays measured in the FRET channel, meaning on the red detector with green excitation light. The legend states the name of the respective FRET pair. For the associated expected FRET efficiency, and corresponding decreased donor lifetime in the presence of the acceptor refer to table 18.1.

The fluorescence decay curves recorded in the FRET channel contain not only the FRET signal, but also fractions of fluorescence emitted by the donor and the directly excited acceptor. Reference measurements with single dyes indicated a spectral bleedthrough of approximately $21 \%$ of the donor fluorescence and a direct excitation of the acceptor by green light of about $16 \%$ compared to direct excitation with red light.

\footnotetext{
${ }^{1}$ also referred to as fluorescence lifetime histograms
} 
In the decay curve on the top left of Fig. 18.1, which was measured in confocal mode, it is evident that the donor originally has a monoexponential decay (green curve) as expected. The lifetime decrease of the donor fluorescence with increasing FRET efficiency is visible in the top left graph. FRET pairs 79 and 80 appear to follow a biexponential decay with a fast FRET component and a slow component that is approximately the same as the original donor lifetime. This could be due to the presence of single donors where either labeling of the DNA was incomplete or the acceptor bleached. The confocal fluorescence decay curves of the donors were fitted with a single or if necessary a double exponential decay in the software Origin. The resulting decay times are stated in table 18.1. The lifetime without FRET is shorter than the $3.8 \mathrm{~ns}$ specified by the manufacturer, but that could be due to the chemical coupling to DNA. If a lifetime of 3.33ns corresponds to 5\% FRET efficiency, the original lifetime would be 3.51ns. Due to FRET, the lifetimes clearly do decrease, but not as strongly as expected. If an original lifetime of $3.51 \mathrm{~ns}$ is assumed, then $0.82 \mathrm{~ns}$ would correspond to $77 \%$ FRET efficiency. When the donors are exposed to STED light, a short drop of the decay curve directly after excitation appears, as expected.

The confocal signal acquired in the FRET channel in the top right graph shows that the acceptor originally has a monoexponential decay (green curve). With FRET, depending on the efficiency, the curve is either narrower and peaks earlier or broader and peaks a little bit later. When measurements with STED are performed, the shape of the curves shows the same features that were seen in simulations in Fig. 11.1: an initial peak due to excitation, then a sharp decrease caused by STED and afterwards, a second peak or shoulder due to ongoing FRET from the donor. Here, the difference between FRET pairs is actually more obvious than in the confocal data. Unfortunately, since there is no explicit fit function for the acceptor lifetime curve as explained in section 10.1, the FRET rate cannot directly be deduced from the data.

In the second right hand graph in Fig. 18.1, one can see that the most efficient FRET (blue curve) has to a significant part already happened during the STED pulse, therefore, the fewest counts are registered after depletion, in qualitative agreement with the simulations. In case of medium FRET, a second peak appears, which for lower FRET is less pronounced and broader, as anticipated. When comparing the second and third graph on the right side of Fig. 18.1, one can see that an increase of the STED intensity causes a sharper decrease of the fluorescence due to depletion, but that the order of the curves, i.e. the relative amount of fluorescence emitted by different FRET pairs afterwards, is not changed. Between the lowest and second lowest right hand graph, the delay between excitation and STED pulse was shortened. This changes the order of the curves, i.e. the relative amount of fluorescence from different FRET pairs. One can see that from the most efficient FRET pair, more counts were registered, because depletion ended earlier. This agrees with expectations from simulations, as shown in Fig. 11.3. Due to the shorter delay, it is more obvious that the more efficient 


\begin{tabular}{|l|c|c|c|c|c|c|c|c|}
\hline name & $\mathbf{E}_{\text {FRET }}$ & $\tau_{\text {DA }}(\mathbf{n s})$ & $\mathbf{t}_{\mathbf{1}}(\mathbf{n s})$ & $\mathbf{s . e . 1}(\mathbf{n s})$ & $\mathbf{A}_{\mathbf{1}}(\mathbf{a . u})$. & $\mathbf{t}_{\mathbf{2}}(\mathbf{n s})$ & $\mathbf{s . e . 2}(\mathbf{n s})$ & $\mathbf{A}_{\mathbf{2}}(\mathbf{a . u})$. \\
\hline DNA 79 & 0.93 & 0.27 & 0.82 & 0.07 & 351,801 & 3.43 & 0.16 & 91,881 \\
\hline DNA 80 & 0.69 & 1.18 & 1.47 & 0.12 & 873,866 & 4.77 & 1.84 & 119,734 \\
\hline DNA 81 & 0.34 & 2.51 & 2.23 & 0.02 & - & - & - & - \\
\hline DNA 82 & 0.13 & 3.31 & 2.75 & 0.03 & - & - & - & - \\
\hline DNA 83 & 0.05 & 3.61 & 3.33 & 0.04 & - & - & - & - \\
\hline
\end{tabular}

Tab. 18.1: Single molecule FRET DNA constructs (described in table 15.1), their theoretically expected FRET efficiency $E_{\mathrm{FRET}}$ and corresponding lifetime $\tau_{\mathrm{DA}}$ (calculated in table 10.1) are stated. The fluorescence decay curves determined from confocal measurements (shown in Fig. 18.1) were fit with a single $\left(t_{1}\right)$ or double exponential decay fit (decay times $t_{1}, t_{2}$, amplitudes $A_{1}, A_{2}$ ) in Origin as necessary, the standard error (s.e.) of the fit is given.

FRET pair (red curve) peaks earlier than the less efficient FRET pair (yellow curve), as anticipated.

In general, the progress of the measured curves does not vary as distinctly as that of the simulated curves, the maxima are flatter and the slopes in the histogram's last section not as different. This is due to the fraction of fluorescence from the directly excited acceptor and bleedthrough of the donor contained in the curves in addition to the FRET signal, that was mentioned above.

Exemplary decays of FRET construct DNA 79, which were accumulated for different STED intensities while keeping the pulse delay constant, are shown in Fig. 18.2. As expected from simulations shown in Fig. 11.2, a heightened STED intensity leads to a stronger decrease of fluorescence during the STED pulse. Due to the increasing depletion of the donor, less energy can be transferred to the acceptor after the STED pulse, so that the area under the acceptor's curve decreases. The depletion of the donor is slightly more efficient than it was simulated.

The STED pulse delay was changed in steps of 320ps while keeping the intensity constant. Histograms of FRET construct DNA 79 that were accumulated for each delay value are shown in Fig. 18.2. For a conversion of the delay values in the figure legend from a.u. to ns, refer to table 14.1. In agreement with the simulations shown in Fig. 11.3 , the sharp decrease of photon counts due to depletion occurs later for larger delays. If the delay increases, the amount of fluorescence detected in the FRET channel after the STED pulse decreases, because a larger part of the energy transfer already happened before or during the pulse. Fluorescence decay curves of all five FRET constructs are shown in supplementary Fig. 28.2 for different STED intensities and in supplementary Fig. 28.3 for different STED delays. 

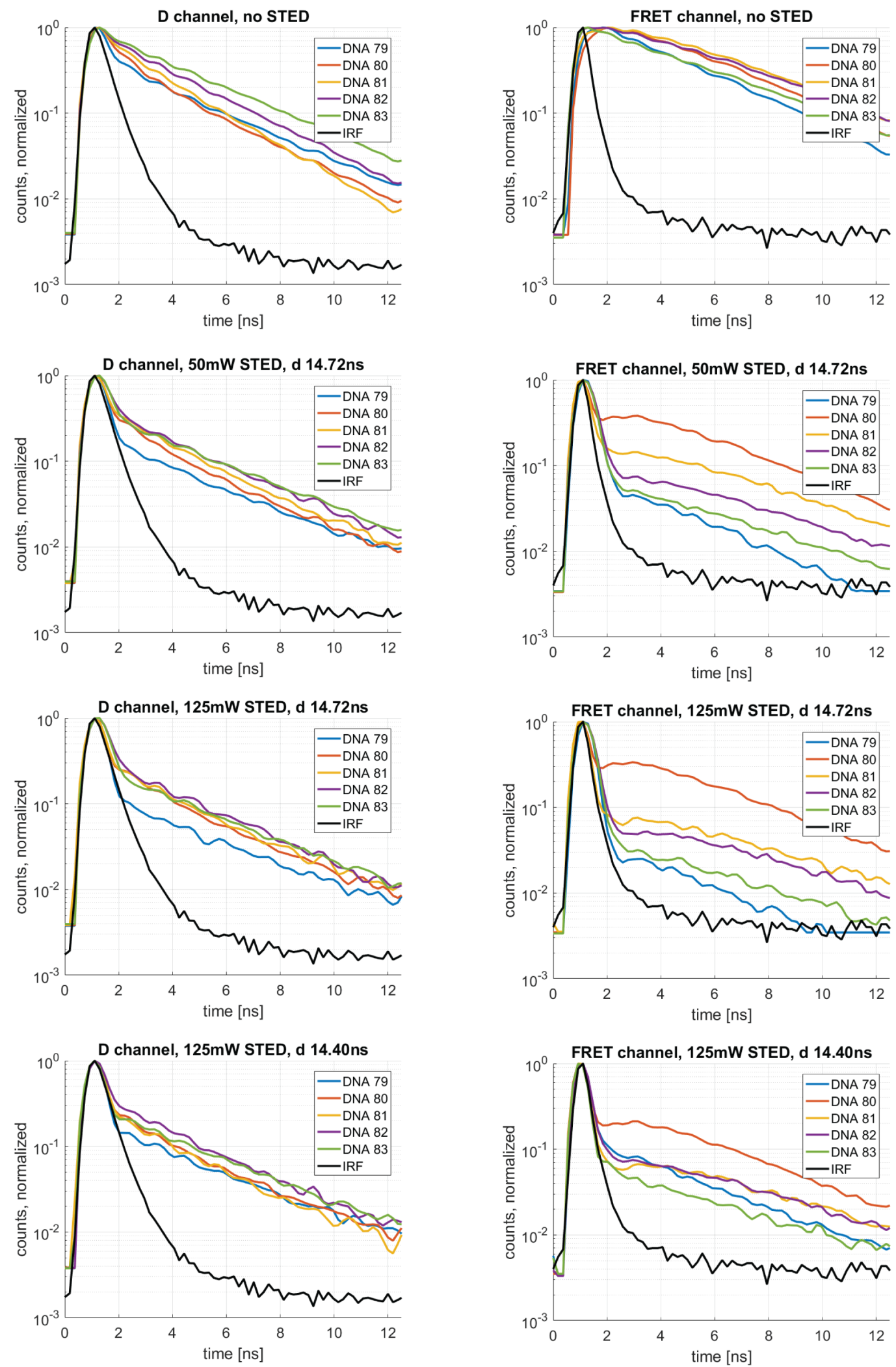

Fig. 18.1: Fluorescence decay curves accumulated in donor and FRET channel, i.e. with green excitation in the green or red detection channel, with different STED parameters from several single molecule FRET constructs, detailed in table 15.1. 

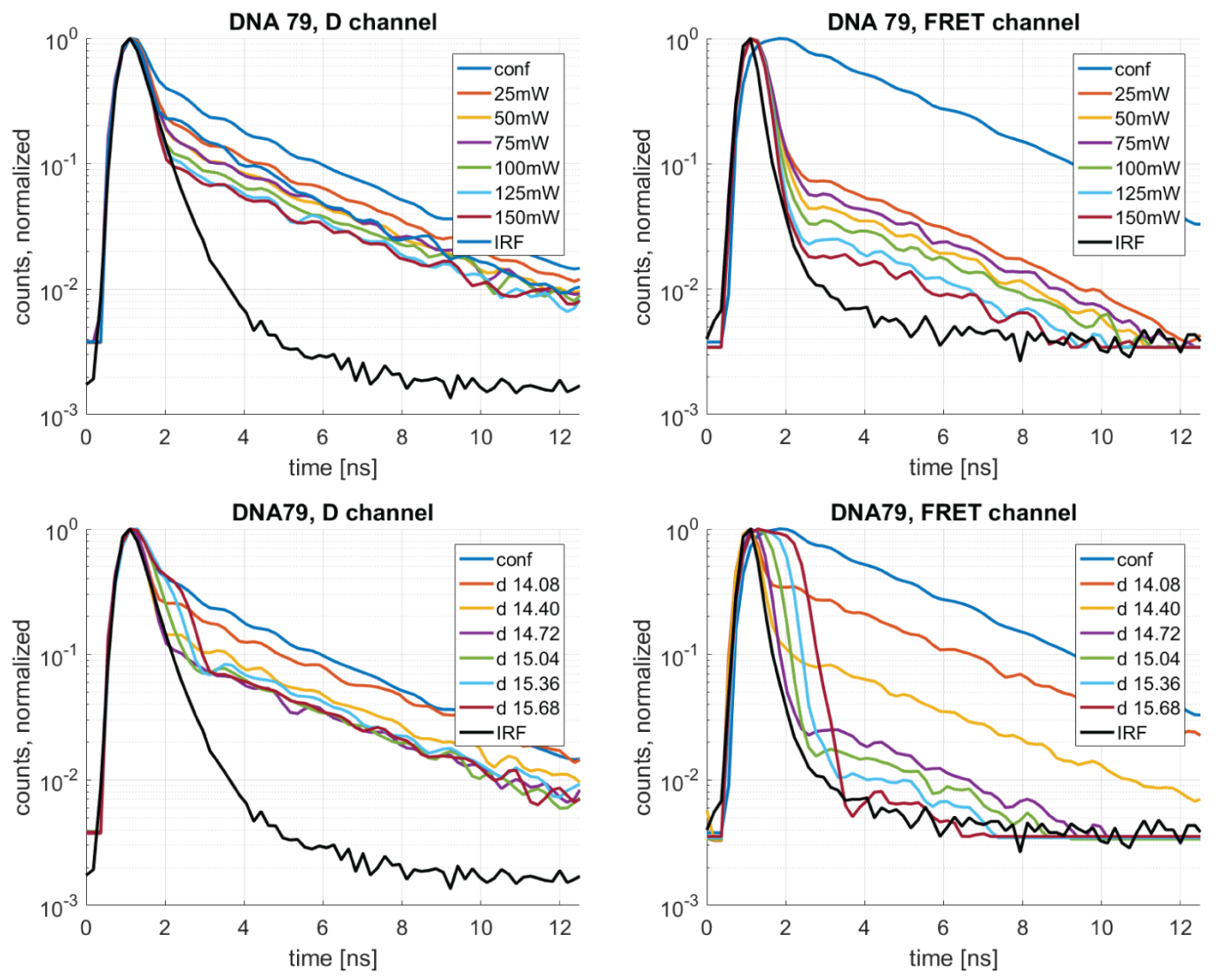

Fig. 18.2: Fluorescence decay curves accumulated in donor and FRET channel for FRET construct DNA79. Top: The STED intensity was varied while keeping the pulse delay constant. Bottom: The STED pulse delay was increased in 320ps steps while keeping the STED intensity constant. The legend states the delay value in ns. All histograms were deconvolved with the measured IRF and normalized. 


\subsection{Summary and discussion}

All in all, the measured and simulated fluorescence decay curves agree very well with each other. All features seen in the progress of the curves and the effects of changing STED intensity and delay are comparable. In conclusion, the simple photophysical model for the simulations is sufficient to explain the combined effects of FRET and STED observed in measurements. No additional higher excited states or triplet states and no further electronic transitions were required.

As was mentioned before in section 10.1, no explicit fit function for the complete acceptor histogram is available. Yet, the shape of the decays is noticeably different for each FRET pair. Thus, one could think of employing a pattern matching approach for identification. This approach will be further discussed in section 20.3. The donor lifetime histogram recorded for each pixel can of course be fit with a single exponential decay function to extract the FRET efficiency. However, the donor image is not superresolved.

Experimentally acquired fluorescence decay curves in the FRET channel contain contributions from donor bleedthrough and directly excited acceptor emission. Both have a known decay time and can thus be accounted for in a data analysis model which unmixes the different decay components. 


\section{Differing spatial resolution}

The aim of the experiments presented in this chapter was to maximize the spatial resolution in the FRET channel by optimizing the STED parameters. In lieu of the spatial resolution, the FWHM of images of single FRET pairs was used. Since both dyes are $10 \mathrm{~nm}$ or less apart, they are regarded as a point source here and their images are fitted with symmetric 2D Gaussian functions.

The workflow for determining the FWHM of FRET pairs for given illumination conditions is outlined in Fig. 19.2. A confocal overview scan of the single molecule sample was performed to locate FRET pairs. Then a small region centered on each pair was scanned with $10 \mathrm{~nm}$ pixel size, as detailed in section 14.2. Excitation was altered in a line-wise fashion to obtain three images quasi simultaneously: the donor channel, i.e. fluorescence excited by $532 \mathrm{~nm}$ detected on APD4, the acceptor channel, i.e. fluorescence detected on APD6 after 612nm excitation and the FRET channel, which contains fluorescence acquired on APD6 after 532nm excitation. The donor was not excited by $612 \mathrm{~nm}$ light. The spectra of donor Atto532 and acceptor Star635P in relation to the spectral detection bands of APDs 4 and 6 as well as the excitation and STED wavelengths are depicted in Fig. 19.1. One exemplary image measured in each channel is shown in the top row of Fig. 19.2. One can clearly see that in this instance two acceptors are located next to each other, but only one is paired with a donor, therefore only one peak is visible in the FRET channel.

In each sample containing one type of FRET pairs, more than 150 small ROIs were scanned for each set of STED parameters. The raw data was acquired with TCSPC and subsequently time gated in order to reduce early emission and background from stray light. Only photons arriving between 2.2ns and 9.6ns (compare to Fig. 18.1) contributed to the intensity images which were then analyzed. The FRET channel was corrected for bleedthrough by subtracting $21 \%$ of the donor channel's intensity and for direct excitation by subtracting $16 \%$ of the acceptor channel's intensity as depicted in supplementary Fig. 28.4. The correction factors were obtained from measurements with singly labeled reference DNA constructs.

The center of each fluorescence peak in the acceptor channel, which has the highest resolution, was determined by fitting it with a 2D Gaussian function in MATLAB, refer to supplementary section 29 . Then, the images in all channels were shifted and 


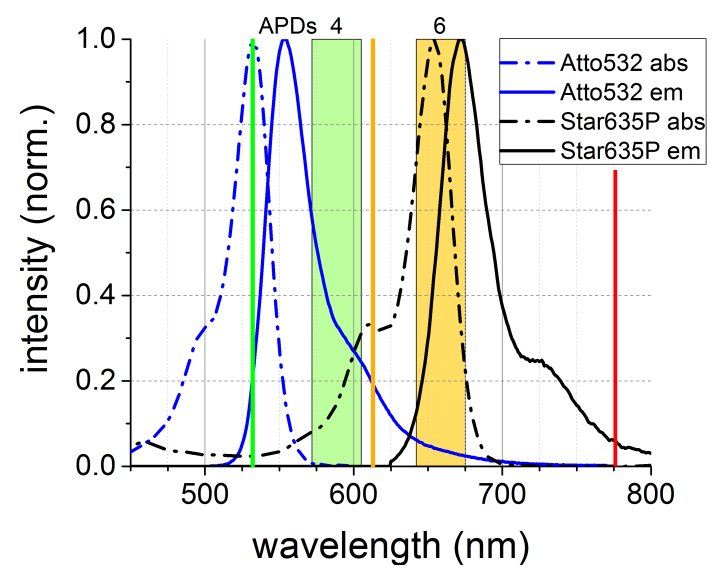

Fig. 19.1: Normalized absorption and emission spectra of Atto532 and Star635P plotted over the spectral windows of detection channels APD4 and APD6. Excitation wavelengths of $532 \mathrm{~nm}$ and $612 \mathrm{~nm}$ as well as the STED wavelength of $775 \mathrm{~nm}$ are indicated.

overlaid accordingly as depicted in the second row of Fig. 19.2 ${ }^{1}$. Concurrently, a mask was generated, which contained the information about how many individual images contributed to each pixel of the summed image. The image stack was summed up and the result is displayed in the third row. Both the summed images and the mask, which acted as a weight for each pixel, were used to fit the summed images again with a 2D Gaussian function. In the last row of Fig. 19.2, this Gaussian model function is shown. The associated MATLAB code is given in supplementary section 29.

For each set of STED parameters, the FWHM of the Gaussian fit to each image channel was noted. The resulting plots in Fig. 19.3 show the dependence of the FWHM on the STED intensity and delay. The length of the error bars corresponds to the standard deviation of the fit of the acceptor images. Since the acceptor is excited directly with $612 \mathrm{~nm}$, its FWHM value must theoretically be the same for each FRET construct. 300nm were set as the upper bound of the STED image FWHM fits. Values of the FWHM below $50 \mathrm{~nm}$ are not realistic at the employed STED intensities, they correspond to cases where the fit wrongly converged on bright noise pixels.

For the same illumination conditions, different FWHM values are obtained in each channel, as can be seen in Fig. 19.3 and the bottom row of Fig. 19.2. The FWHM is used as a substitute for the spatial resolution. The donor's fluorescence image is nearly diffraction limited, with a FWHM of at least 150nm, which is expected due to its low depletion efficiency. The acceptor channel, in the bottom row of Fig. 19.3, shows the maximum resolution achievable for the given STED parameters. The resolution increases for increasing STED intensity as anticipated and reaches values below 100nm. It should be the same FWHM for each FRET construct, because the acceptor is excited directly. The FWHM of the FRET signal is usually in between the donor's and the

\footnotetext{
${ }^{1}$ Images containing less than 200 photons were not included in the analysis.
} 
Donor channel, exc 532nm
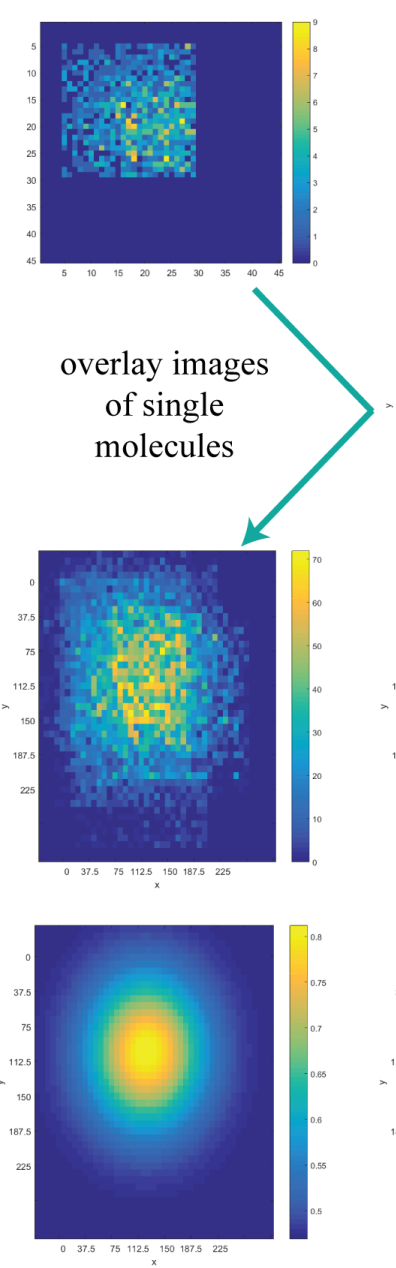

Acceptor channel, exc $612 \mathrm{~nm}$
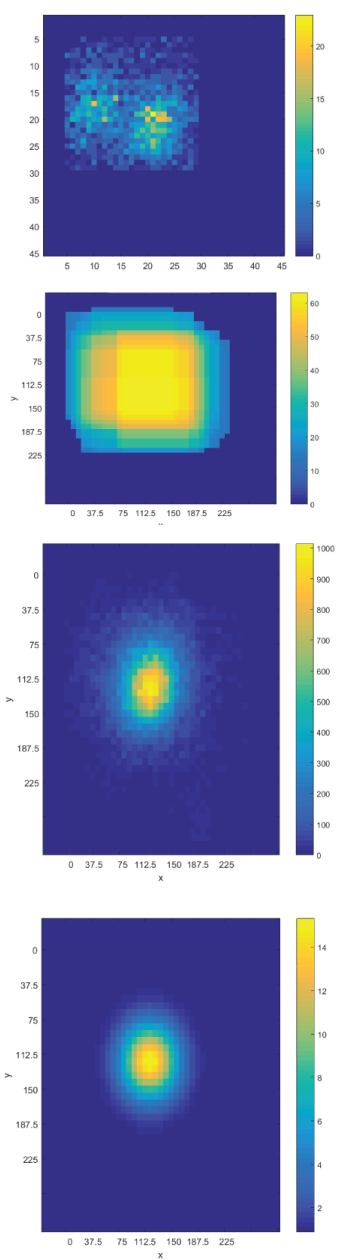

Acceptor channel,

exc 532nm

$=$ FRET channel

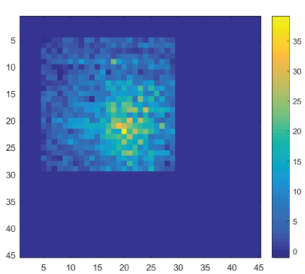

fit with

2D Gaussian function

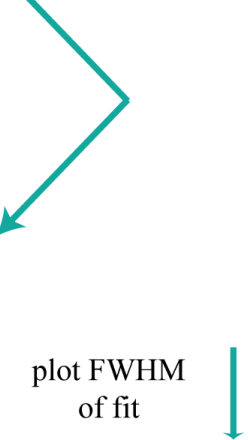

Fig. 19.2: Determining the FWHM dependence on different STED parameters. Scans of more than 150 small ROIs were centered, overlaid and summed. Weights for fitting were determined according to the overlay, and used to fit the summed images with a $2 \mathrm{D}$ Gaussian model function. Resulting FWHM values of the Gaussian fit are plotted in Fig. 19.3. 

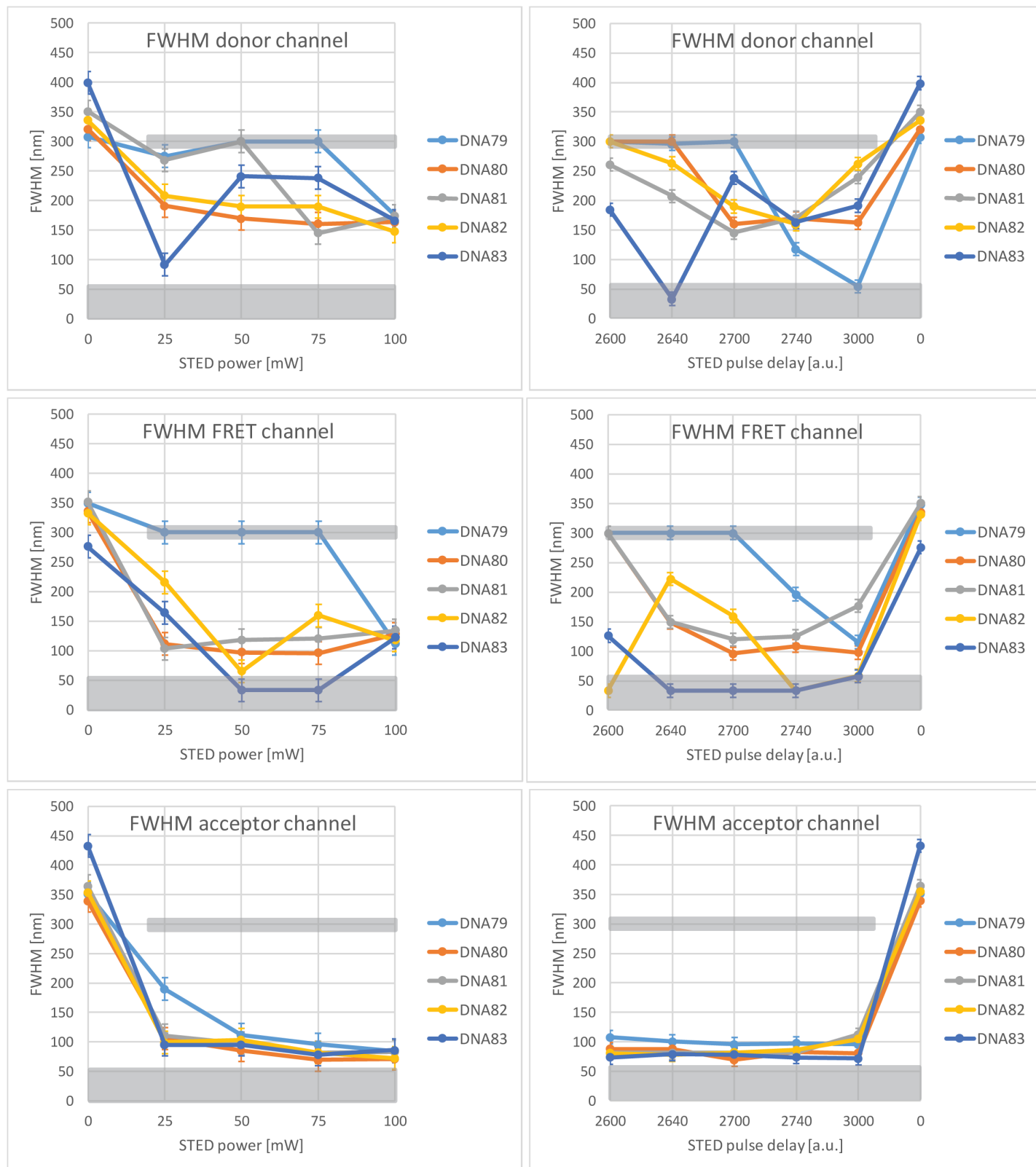

Fig. 19.3: FWHM of a 2D Gaussian function fitted to the summed overlay of many small ROI scans with appropriate weights. Over 150 scans were conducted for each set of STED parameters in each FRET sample. Gray areas: values where fit converged on noise, $300 \mathrm{~nm}$ was set as upper bound for the STED scan fits. 

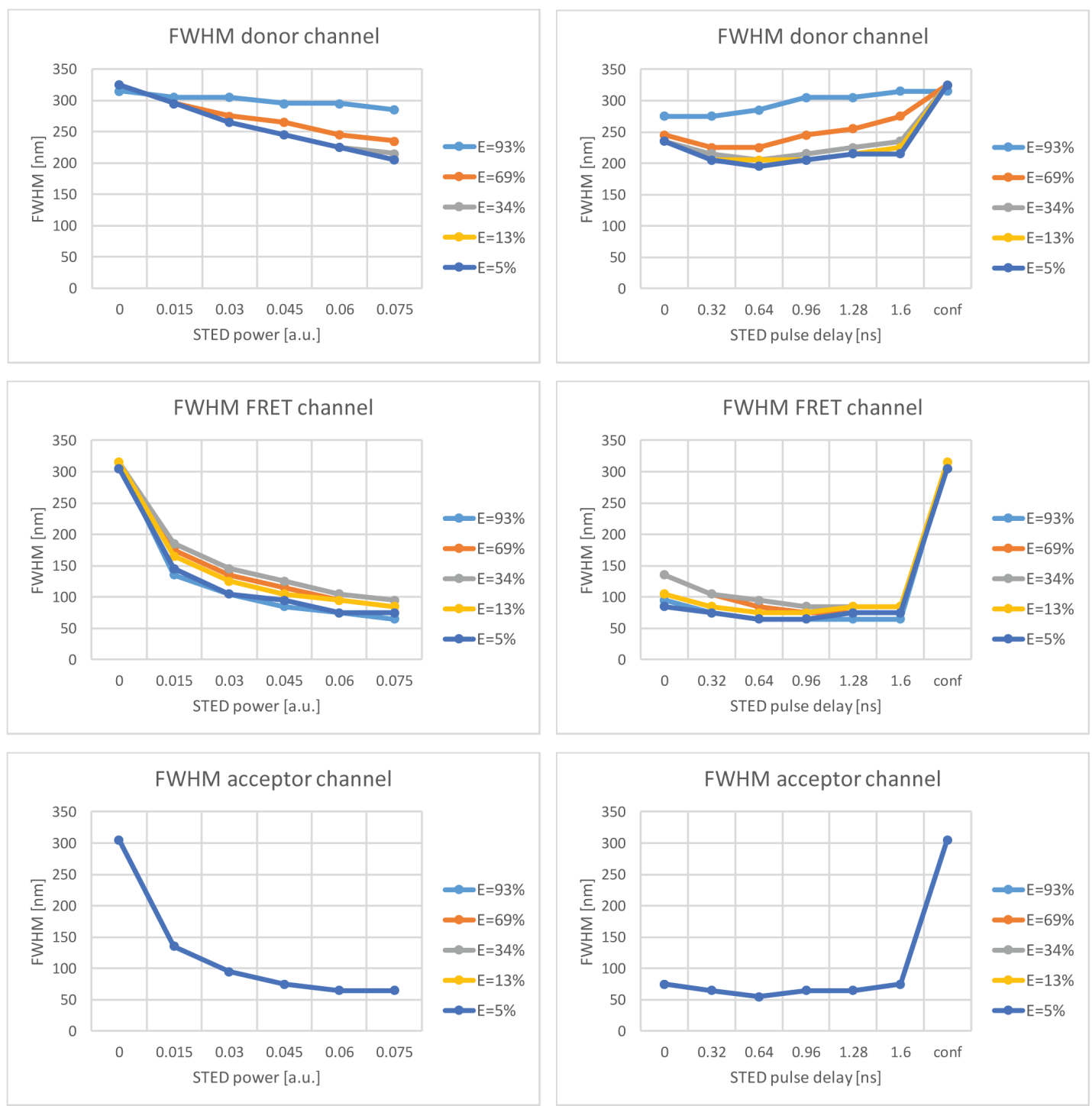

Fig. 19.4: FWHM calculated from simulations with the stated FRET efficiencies E for different STED powers and pulse delays. These values of E are the theoretically expected values for DNA constructs 79 to 83.

acceptor's. This is because the acceptor is depleted efficiently by the STED pulse, but can subsequently emit again after late energy transfer from the donor, as detailed in chapter 11.5. In order to show that this lower resolution in the FRET channel is indeed no measurement artifact, the FWHM dependence was calculated from simulations. The resulting curves are plotted in Fig.19.4 and match the measured curves rather well. One can see that the resolution in the FRET channel increases with the STED intensity. A good compromise value for the STED pulse delay can be found, which is slightly larger than what would be chosen for the acceptor alone. 


\subsection{Summary and discussion}

In this section, the FWHM of single FRET pairs imaged simultaneously in donor, acceptor and FRET channel was determined in lieu of the spatial resolution for different STED parameters. The donor's fluorescence remains almost diffraction limited with a FWHM of at least 150nm. The FWHM of the directly excited acceptor decreases with increasing STED intensity to approximately $75 \mathrm{~nm}$. As expected from simulations shown in chapter 11.5 and Fig. 19.4, the FWHM of the FRET signal decreases as well, but only to approximately 100nm. The optimal STED pulse delay depends slightly on the FRET efficiency, as can be seen in Fig. 19.4. However, a good compromise for all FRET pairs can be found at a delay that is a bit longer than what would be suitable for the acceptor alone.

The difference of spatial resolution in the image channels leads to artifacts, if one tries to calculate the FRET efficiency in a pixel-wise manner. This will be discussed in detail in the following chapter 20.

In chapter 11.5, time gating was discussed as a method to improve the spatial resolution by using only photons detected during and shortly after the STED pulse. I applied the same time gates which are shown in Fig. 11.4 to this data set to generate intensity images only with the photons detected during each gate. These images were overlaid, summed and fitted as described above to obtain the FWHM. However, the low photon counts and thus low SNR deteriorated the images, such that no clear resolution improvement could be demonstrated. Significantly more signal would need to be obtained from each molecule pair during the time gate in order to generate high resolution FRET images from photons emitted only during the STED pulse. 


\section{Finding a measure for the FRET efficiency compatible with STED}

\subsection{Obstacles to using established FRET measures on STED data}

In general, the FRET efficiency can be deduced either with fluorescence intensity based methods or with lifetime based methods, see also chapter 6.2. Since the microscope used in this thesis work features a filter-based detection and several excitation wavelengths, ratiometric FRET imaging would be a straightforward intensity-based quantification method. However, when STED is used, two issues arise. First, the donor, FRET and acceptor channels differ in spatial resolution, which was demonstrated in the previous chapter 19 . Therefore, image artifacts arise if one calculates intensity ratios for individual pixels, because the acceptor channel features sharper edges. One could create a mask from the high-resolution acceptor image before calculating ratios only in certain areas. But the donor fluorescence inside the masked area would still contain contributions from surrounding areas due to diffraction. This is illustrated in Fig. 20.1. Second, donor fluorophores are depleted less efficiently than acceptors by STED, as was presented in chapters 18 and 11 . Hence, the intensity ratio of donor and acceptor in the same instance will always be different in STED and confocal images. Furthermore, the amount of acceptor depletion and thus the intensity ratio will depend on the STED intensity and pulse delay, which will be detailed in the following section 20.2.

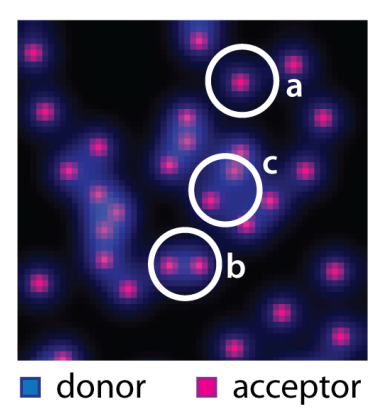

Fig. 20.1: Simulated overlay of donor and acceptor fluorescence images, which differ in spatial resolution. White circles indicate the detection area from which the signal is integrated at different scan positions: a) Signal from one FRET pair. b) Signal from two adjacent FRET pairs. c) Signal from two acceptors and five donors. 
Alternatively, since the microscope is also capable of TCSPC, the FRET efficiency could be deduced from the fluorophore's lifetime. Usually, the donor lifetime histogram can be fit with an exponential decay function. The FRET efficiency is calculated from the ratio of the donor's lifetime in the presence and absence of the acceptor. Unfortunately, the donor image is almost diffraction-limited. Again, creating a mask of the acceptor distribution before analyzing the donor lifetime in that area is no true solution, because the lifetime histogram will contain signal from the surrounding areas due to diffraction. Consequently, superresolution information and functional information via FRET could not be obtained from the same data. Instead, it would be advantageous to extract the FRET efficiency from the acceptor lifetime histogram. However, no explicit analytical fit function is available, as was investigated previously in section 10.1. Pattern matching approaches could offer an interesting approach and will be examined in section 20.3.

\subsection{Normalizing to the acceptor emission}

In the previous section, the problem was posed that the differing spatial resolution of donor and acceptor channel leads to image artifacts when using ratiometric methods to calculate the FRET efficiency $E_{\mathrm{FRET}}$ :

$$
E_{\mathrm{FRET}}=\frac{I_{\mathrm{FRET}}^{A}}{I^{D}+I_{\mathrm{FRET}}^{A}}
$$

Instead of comparing the sensitized acceptor emission $I_{\mathrm{FRET}}^{A}$ to the quenched donor emission $I^{D}$, one could compare it to the directly excited acceptor emission $I_{\mathrm{dir}}^{A}$ :

$$
R_{\mathrm{FRET}}=\frac{I_{\mathrm{FRET}}^{A}}{I_{\mathrm{dir}}^{A}}
$$

Fortunately, the spatial resolutions of acceptor and FRET channels can be rendered almost equal with optimized STED parameters, and a donor with a short lifetime (section 11.6). The fluorescence intensity in the acceptor channel is of course proportional to the number of acceptor molecules and if the excitation intensity is constant, the signal can be used for normalization: more sensitized acceptor emission would correspond to a higher intensity ratio.

The intensity ratios $E_{\text {FRET }}$ and $R_{\text {FRET }}$ of fluorescence emitted by the previously introduced single molecule FRET constructs were calculated for confocal and STED measurements. The resulting ratios for measurements versus STED intensity and pulse delay are plotted in Fig. 20.2. Unfortunately, the s.d. is rather large, probably due to the low photon counts. Hence, the same intensity ratios were calculated from simulated data and are shown in Fig. 20.3. 
The absolute values of $E_{\mathrm{FRET}}$ determined from experimental data and simulations do not fully agree with each other and with the theoretically expected values stated in the legend of Fig. 20.3. However, the proportions are reproduced as expected, i.e. DNA 79 has the highest FRET efficiency, etc. The same is observed for $R_{\mathrm{FRET}}$ : the absolute values derived from simulation and experiment differ, in this case because the direct excitation of the acceptor was stronger in the experiment. Yet, the curves match reasonably well within the error margins. Most importantly, it is evident that both measures depend both on the STED intensity and delay, i.e. the same FRET construct appears to have a different FRET efficiency under different illumination conditions. Thus, both intensity ratios are not a reliable measure for the FRET intensity in STED images.

The reason that $R_{\text {FRET }}$ also correlates with the STED parameters is that the directly excited acceptor intensity $I_{\text {dir }}^{A}$ simply decays either by stimulated or spontanous emission, whereas the amount of sensitized acceptor emission $I_{\text {FRET }}^{A}$ during and after the STED pulse depends on the STED intensity and delay. This can be seen in the lifetime histograms in chapter 18, when comparing the curves of DNA 83 (no FRET) with the curves of the other FRET constructs. The detected intensity corresponds to the area under the curve.

\subsubsection{Combining intensity ratio and photon arrival time}

It was discussed in previous sections that $E_{\mathrm{FRET}}$ is not suitable for calculating the FRET efficiency in STED images, because the donor channel, being depleted much less, has a significantly lower resolution than the FRET and the acceptor channel. Instead, $R_{\text {FRET }}$ was proposed as a measure, because FRET and acceptor channel can have almost the same resolution if the experimental parameters are adjusted. However, the intensity emitted in both channels depends on the STED settings each in a different way. Hence $R_{\text {FRET }}$ also correlates with them, which can be seen in Fig. 20.2 and Fig. 20.3. Most importantly, the curves of $R_{\text {FRET }}$ cross each other, meaning that under certain conditions FRET pairs with different transfer rates could not be distinguished.

To overcome this uncertainty, it was proposed to combine $R_{\mathrm{FRET}}$ with lifetime information to better identify different FRET pairs. The first moment of the acceptor lifetime histograms, the mean photon arrival time $E_{\mathrm{tA}}$, was used. In case of high FRET, more photons arrive earlier, whereas in low FRET, photons arrive later, as can be estimated from the histograms shown in Fig. 28.3.

In order to test if the different FRET constructs could be reliably distinguished by a combination of $R_{\mathrm{FRET}}$ and $E_{\mathrm{tA}}$, both values were calculated for each measured FRET pair. Then, scatter plots were constructed with $R_{\mathrm{FRET}}$ as x-coordinate and $E_{\mathrm{tA}}$ as y-coordinate of each measured data point, which are shown in Fig. 20.4 and Fig. 28.5. The ellipses 


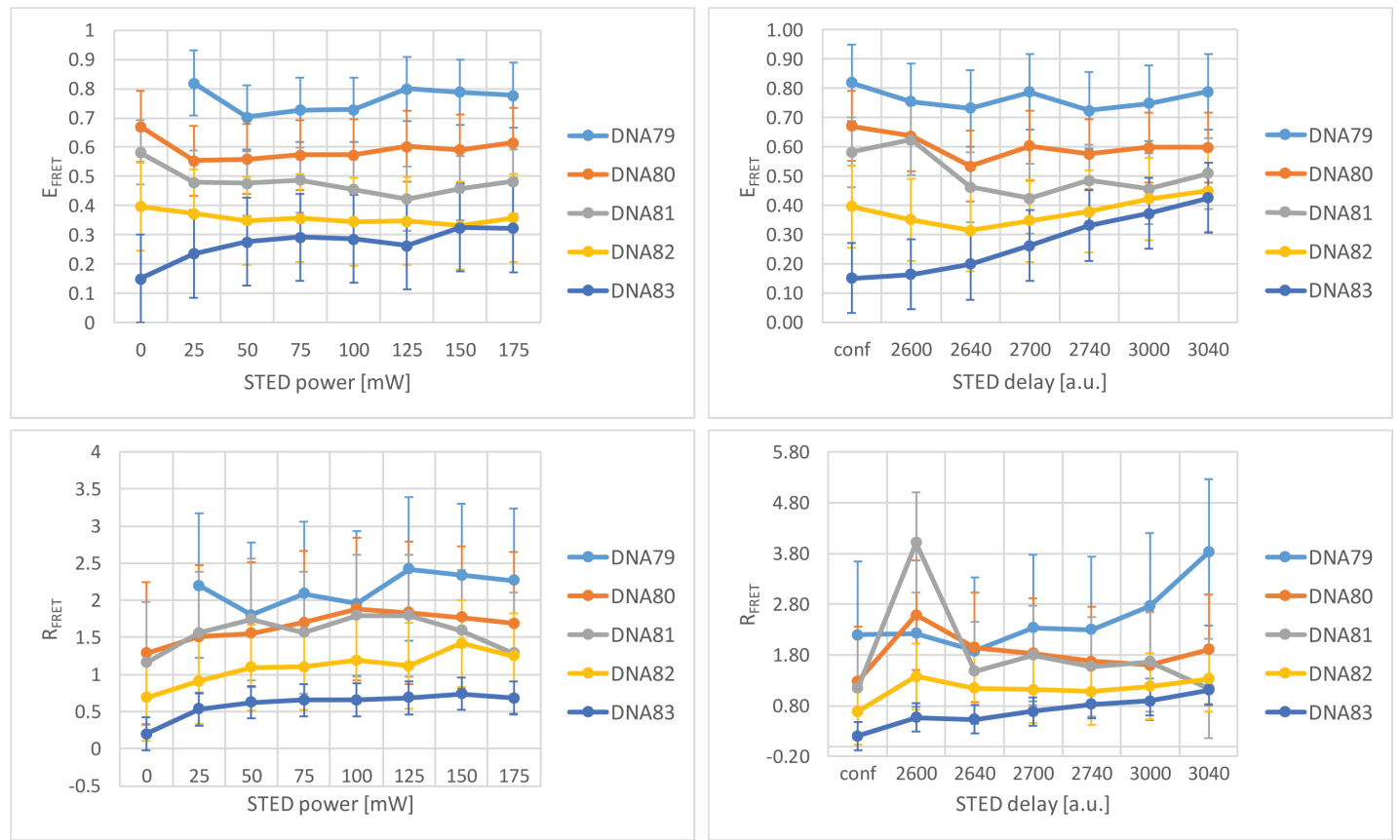

Fig. 20.2: Fluorescence intensity ratios $E_{\mathrm{FRET}}$ and $R_{\mathrm{FRET}}$ were calculated from images of single molecule FRET constructs detailed in table 15.1, which were acquired with different STED powers and pulse delays. Error bars correspond to the s.d. between individual pairs.
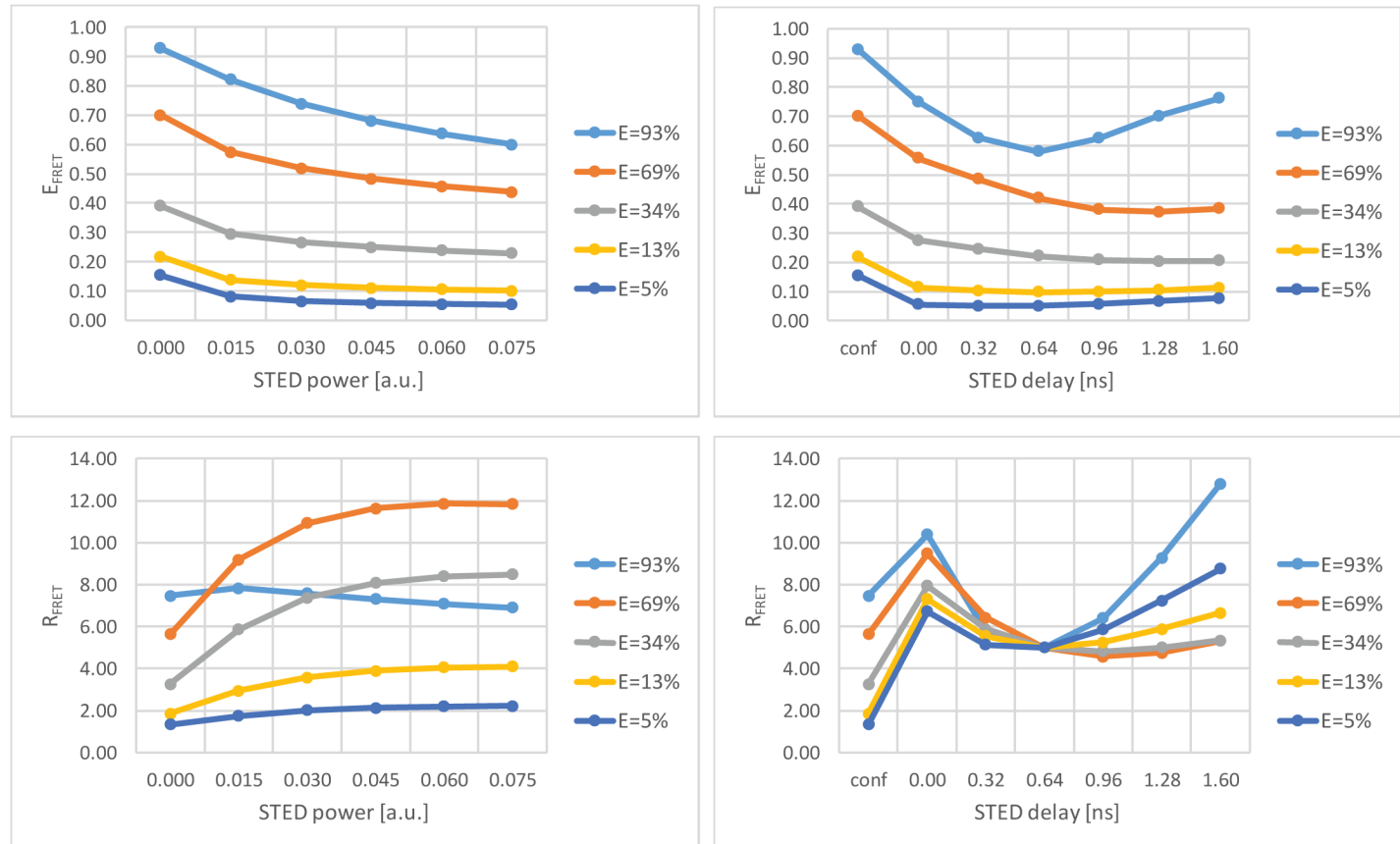

Fig. 20.3: Fluorescence intensity ratios $E_{\text {FRET }}$ and $R_{\text {FRET }}$ were calculated from simulations of single molecule FRET pairs with the same efficiencies as detailed in table 15.1. 
have a diameter of two s.d., meaning they enclose $95 \%$ of the data points belonging to each FRET construct.

In the first graph in Fig. 20.4, one can see that in confocal measurements, the FRET constructs can be discriminated rather well, the s.d. ellipses overlap only a little. Variations of the STED settings change the shape and location of each distribution in the plot. Consequently, the overlap between distributions varies, e.g. at 25mW STED, DNA 80 and DNA 81 almost completely overlap, whereas at 50mW STED, they are distinct, but DNA 81 and DNA 82 completely overlap. In conclusion, the combination of intensity ratio $R_{\text {FRET }}$ and mean photon arrival time $E_{\mathrm{tA}}$ also does not allow a reliable discrimination of the different FRET pairs in STED images. Analogous scatter plots for different STED pulse delays are shown in supplementary Fig. 28.5.

One could try to use not only the first moment (mean), but also the second moment (variance) and maybe the third moment (skewness) to describe the histogram shape and thus distinguish the FRET pairs by their lifetime histograms in addition to their spectral information.

\subsection{Analysis of acceptor decay curves}

Instead of using either a tailfit to extract $k_{\mathrm{FRET}}$, as outlined in section 10.1, or describing the lifetime histogram's shape by statistical moments to identify $E_{\mathrm{FRET}}$, I propose to employ pattern matching. Most importantly, in this method, the complexity of the pattern does not influence the complexity of the analysis. Furthermore, no knowledge of the underlying nature of the decay is necessary. Additionally, pattern matching needs only comparatively few photons for a reliable analysis, in contrast to other methods. It is further discussed in outlook section VII.

In order to employ pattern matching, one first needs reference patterns, i.e. normalized probability density functions that describe how many photons to expect per time interval [41]. These patterns can either be extracted from the data itself in several ways as detailed in [41] and [89], or measured from samples labeled with a single fluorophore or single FRET species of known efficiency.

The raw lifetime histograms should if necessary be binned to increase the photon counts per bin, which helps the algorithm to converge [41], and noise should be reduced. Additionally, the raw data may need to be shifted in time to coincide with the IRF, that could have been measured at a different time. 

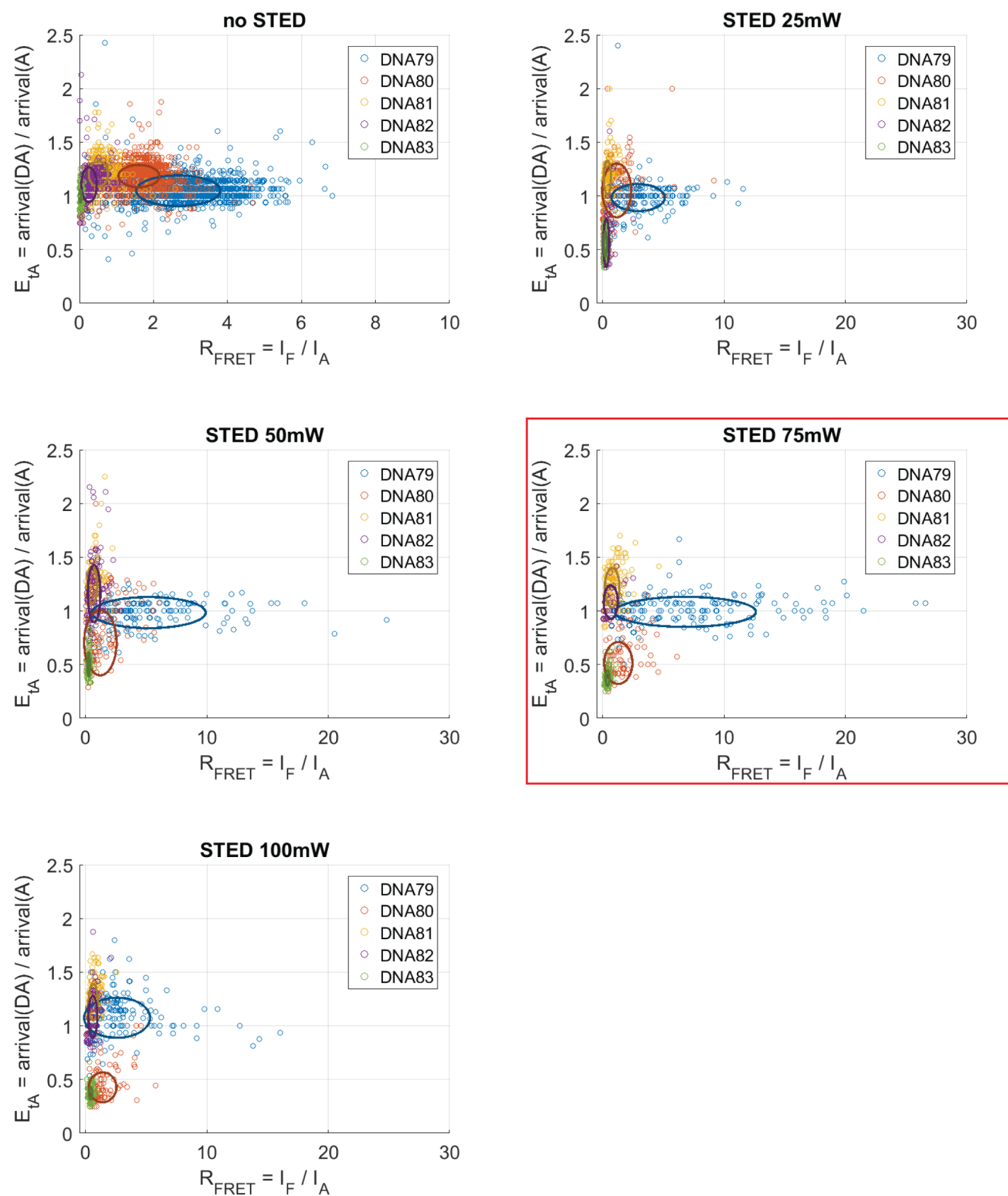

Fig. 20.4: Scatter plots with the acceptor fluorescence intensity ratio $R_{\mathrm{FRET}}$ of emission caused either by FRET $I_{F}$ or by direct excitation $I_{A}$ and the mean photon arrival time $E_{\mathrm{tA}}$ as coordinates for each single FRET pair. Over 150 individual FRET pairs of each FRET construct, detailed in table 15.1, were measured with several different STED intensities. The ellipses have a diameter of two s.d. of each distribution. 
In FRET experiments, any measured lifetime histogram in the FRET channel can contain a superposition of at least four individual patterns:

- sensitized acceptor emission of one or more FRET species

- directly excited acceptor emission with $\tau_{\mathrm{A}}$

- emission of paired donors with $\tau_{\mathrm{DA}}$

- emission of unpaired donors with $\tau_{\mathrm{D}}$

The performance of the algorithm in distinguishing all contributions, especially FRET pairs with similar efficiency, given low photon counts, low SNR or low fraction of one species would have to be tested. However, performing such a test, either experimentally or with simulations, was out of the scope of this thesis. 



\section{Spectral shifting of fluorophores}

During my Diploma thesis work, Franziska Winter and I observed a spectral shift of the fluorescence emission of red fluorophore ensembles to shorter wavelengths during dualexcitation STED imaging. This is described in [142], exemplary intensity distributions in four spectral channels changing over consecutive frames are shown in Appendix E. During my Ph.D. thesis work, I further examined this phenomenon, because it has consequences both for FRET experiments and STED imaging, as will be discussed in chapter 22 and section 21.3.3, respectively. In this chapter, evidence for spectral shifts of the emission of single fluorophores both to shorter (termed blueing) and longer wavelengths (termed redding) is presented. The incidence of the shifts, their magnitude (in $\mathrm{nm}$ ) and the average lifetime of the new species are estimated. I assert that what is observed is photoconversion due to photochemical reactions and not spectral diffusion due to environmental influences, this is further reviewed in section 21.3.1.

\subsection{Confocal measurements of spectral shifts}

This section begins with a short summary of the data acquisition and analysis strategy, which is illustrated in Fig. 21.1. A detailed description of the analysis and results follows.

Single Atto532 or Star635P molecules were immobilized on the surface of a coverslip as depicted in Fig. 16.1. They were imaged in confocal mode in four spectrally adjacent detection windows over many consecutive frames as described in section 14.2. The exact image acquisition parameters are stated in supplementary table 32.2. The absorption and emission spectra of each fluorophore in relation to the spectral detection windows and the excitation wavelengths are illustrated in Fig. 21.2 and Fig. 21.3. The photon counts in each ROI were added up and displayed as an intensity time trace. For each frame the relative intensity distribution in all four detection channels was calculated to better visualize the spectrum of the fluorescence emission. The associated MATLAB code is provided in supplementary section 29.

Spectral shifts of single fluorophores can be observed as sudden jumps between different spectral distributions in the time traces shown in Fig. 21.1. Single step bleaching, i.e. the abrupt drop of the intensity to background level, indicates that indeed only one 


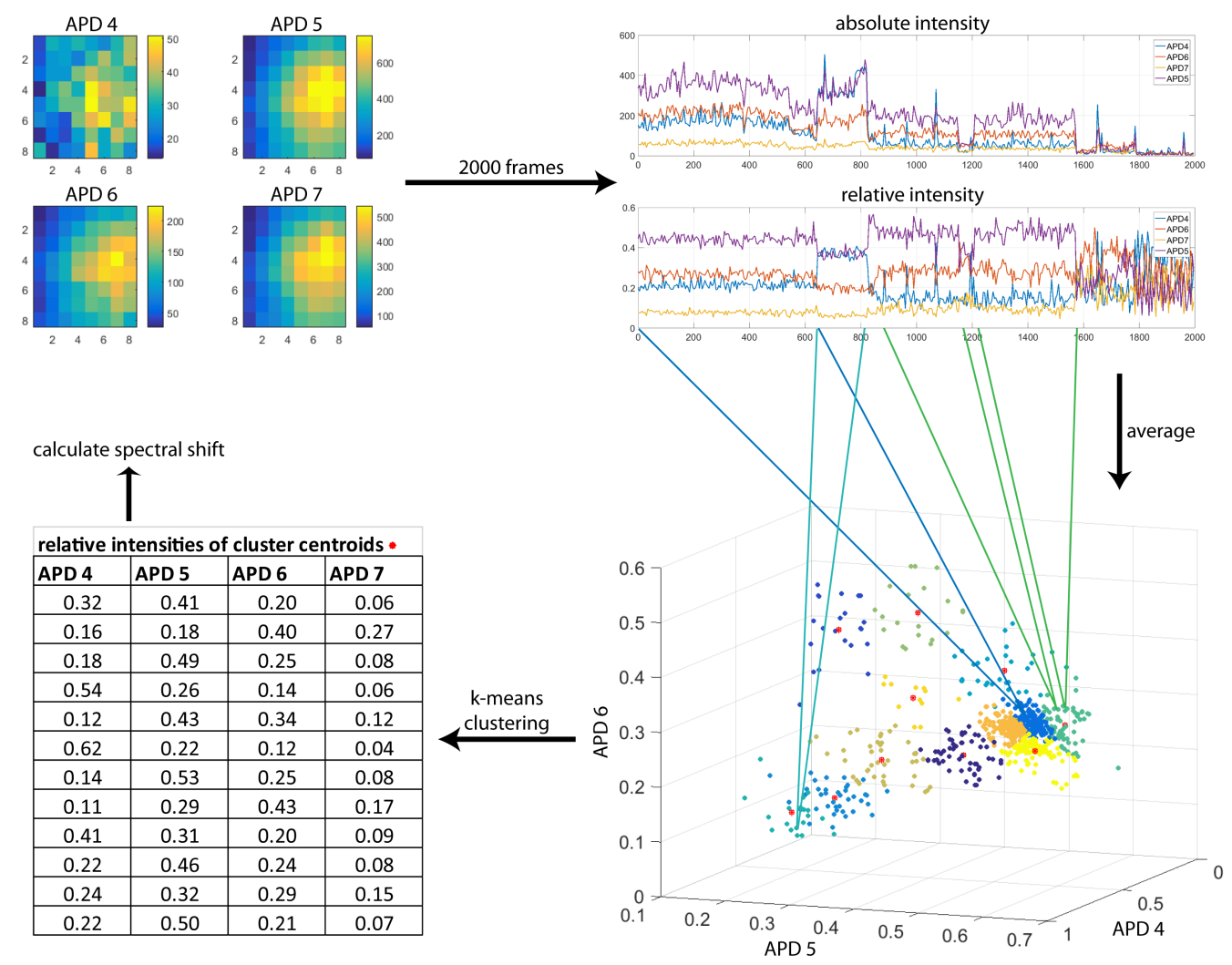

Fig. 21.1: Overview of the analysis workflow of spectral shifts.

fluorescent molecule was present in the scanned area. Therefore, the change of the emission spectrum cannot be due to multiple different fluorophores blinking on and off. In each intensity time trace, one can see the photon count fluctuating in each frame around an average value. This can be due both to noise or to quick blinking of the dyes on a timescale faster than the $5 \mathrm{~ms}$ acquisition time per frame.

Sections of the intensity time trace with the same spectrum were manually selected and the relative intensity in each channel was averaged over time to obtain a single data point with four spectral coordinates. Additionally, the number of frames per section was noted. The corresponding MATLAB code is given in supplementary section 29 . Many such data points, each corresponding to one emission state of a fluorophore, are displayed in a 3D scatter plot in Fig. 21.1. The fourth dimension can be omitted because the relative distribution of the intensity values adds up to one.

A k-means clustering was performed on this data set, the code is provided in supplementary section ??. The number of clusters was preset such that a good separation was achieved while still retaining enough points in each cluster to perform meaningful averaging. The centroid of each cluster, i.e. the mean spectral intensity distribution, was extracted. The spectral coordinates of each centroid were used to calculate the magnitude of the spectral shift compared to the fluorescence emission of the original flu- 

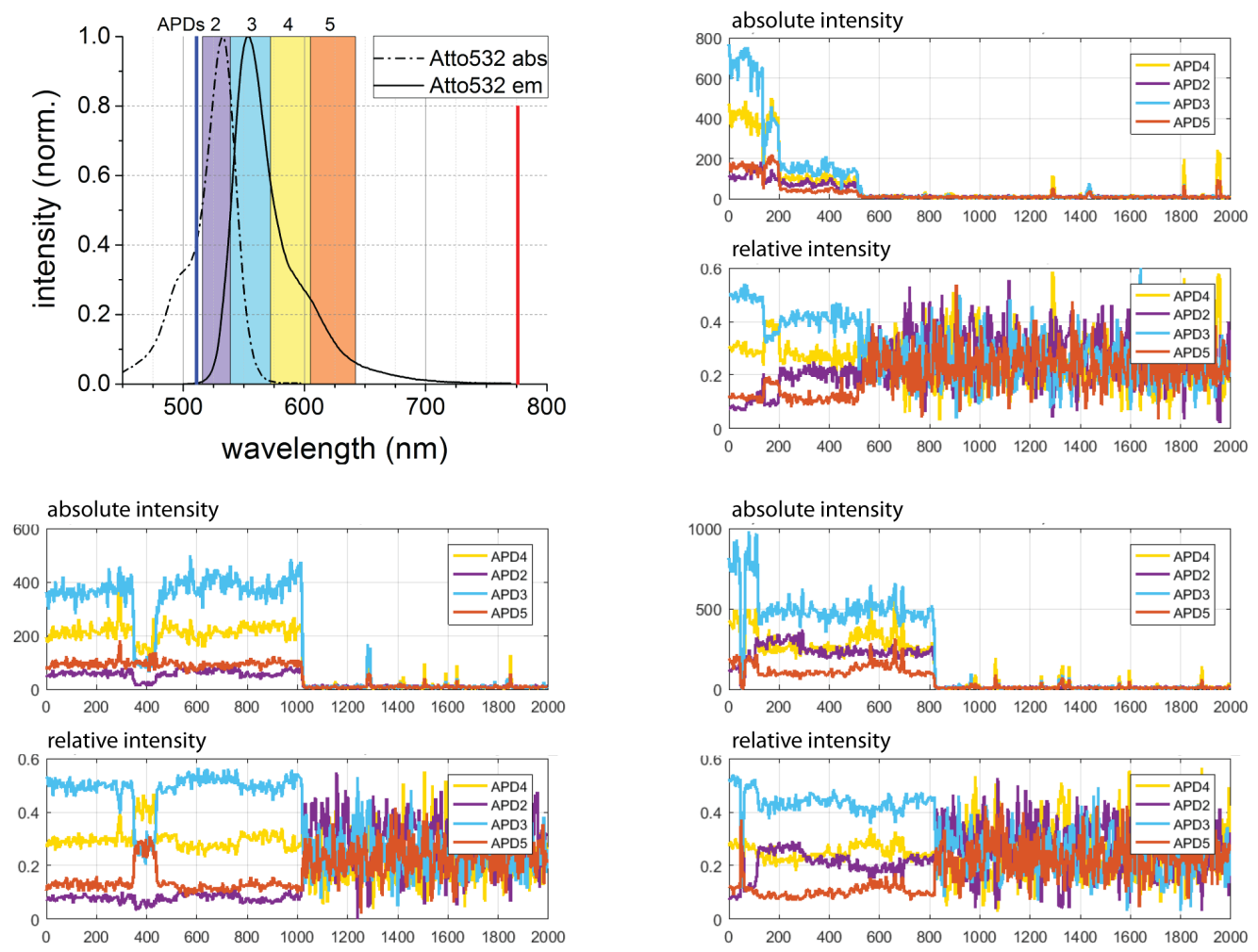

Fig. 21.2: Top left: Absorption and emission spectra of Atto532, plotted over the spectral detection windows of APDs 2 to 5. Excitation was at $510 \mathrm{~nm}$ as indicated by a vertical line, STED at 775nm was not used for these measurements. Besides: Three exemplary fluorescence intensity time traces, each consisting of two plots. The upper plot shows the total photon count per detection channel, the lower plot depicts the relative intensity per detector. The $\mathrm{x}$-axis states the time in ms, corresponding to 400 frames of $5 \mathrm{~ms}$ duration each.

orophore. It was assumed that the shape of the emission spectrum remained unchanged and that each detection channel has the same detection efficiency. Additionally, the number of data points per cluster and the average number of frames of the segments in each cluster were extracted. The MATLAB code is appended in supplementary section 29.

\section{Fluorescence intensity time traces}

In Fig. 21.2, the absorption and emission spectra of Atto532 are plotted over the spectral windows of APDs 2-5, which were used in the measurements. Also depicted are three exemplary intensity time traces, i.e. summed intensity per detection channel over consecutive frames. At each location of a fluorophore, 400 frames of approximately $5 \mathrm{~ms}$ were acquired, totaling to $2000 \mathrm{~ms}$ per trace. The upper plot always contains the absolute photon count on each detector, whereas in the lower plot, the relative 
distribution of the counts per detector is shown in order to facilitate the recognition of spectral shifts.

In the first trace on the top right of Fig. 21.2, three consecutive spectral shifts can be seen: First, at approximately $70 \mathrm{~ms}$, the dye shifts a little to the blue, the intensity on APD 2 increases while that on APD 4 decreases slightly. Then, after about 140ms, the dye undergoes a large red-shift, as can be seen from the marked intensity increase on APDs 4 and 5. Simultaneously, the absolute fluorescence intensity decreases, because the red-shifted photoproduct is excited less efficiently by the 510nm illumination. Later, after $200 \mathrm{~ms}$, the dye converts to a species whose emission is even further blue-shifted than the original. A large fraction of fluorescence is detected on APD 2, while the fractions on APDs 4 and 5 drop. The total intensity emitted by this photoproduct is comparatively low, which could be due either to a reduced absorption coefficient or to a lower QY. Lastly, the fluorophore photobleaches in a single step, with the total intensity dropping to background level.

In the second intensity time trace in the lower left corner of Fig. 21.2, an even more complicated behaviour with a total of seven fluorescent states is observed. First comes a pronounced red-shift at approximately $350 \mathrm{~ms}$, characterized by an intensity drop on APD 3 with a concurrent intensity increase on APDs 4 and 5. This red species emits much less photons in total, probably because it is excited less efficiently by $510 \mathrm{~nm}$. At about 450ms, a series of small spectral shifts with similar total emission starts: First back almost to the original spectral distribution, then a shift to the blue, followed by a slight red-shift to an intermediate state, afterwards another red-shift to a species that resembles state three and finally a clear blue-shift about $950 \mathrm{~ms}$ before single-step photobleaching.

The third intensity time trace also features a total of seven distinct spectral states. After a short excursion to a dark state, the dye shifts slightly to the blue, as seen by the increase of the fluorescence fraction on APD 2. Then, a very large blue-shift occurs at about $100 \mathrm{~ms}$. This is followed by two shifts to the red at $300 \mathrm{~ms}$ and about $470 \mathrm{~ms}$. Another blue-shift occurs at $700 \mathrm{~ms}$, then the dye bleaches in a single step.

In Fig. 21.3, the absorption and emission spectra of Star635P are plotted over the four spectrally adjacent detectors APDs 5-8. The fluorophores were excited with 552nm light, STED at $775 \mathrm{~nm}$ was not used for the measurements described here. STED measurements of single Star635P molecules are described in the following section 21.2. Besides, three exemplary intensity time traces of single Star635P molecules are shown. As described above, 400 frames of about $5 \mathrm{~ms}$ each were acquired at every fluorophore location, totaling to $2000 \mathrm{~ms}$ traces. The upper plot always contains the absolute photon count on each detector, whereas in the lower plot, the relative distribution of the counts per detector is shown in order to facilitate the recognition of spectral shifts. 

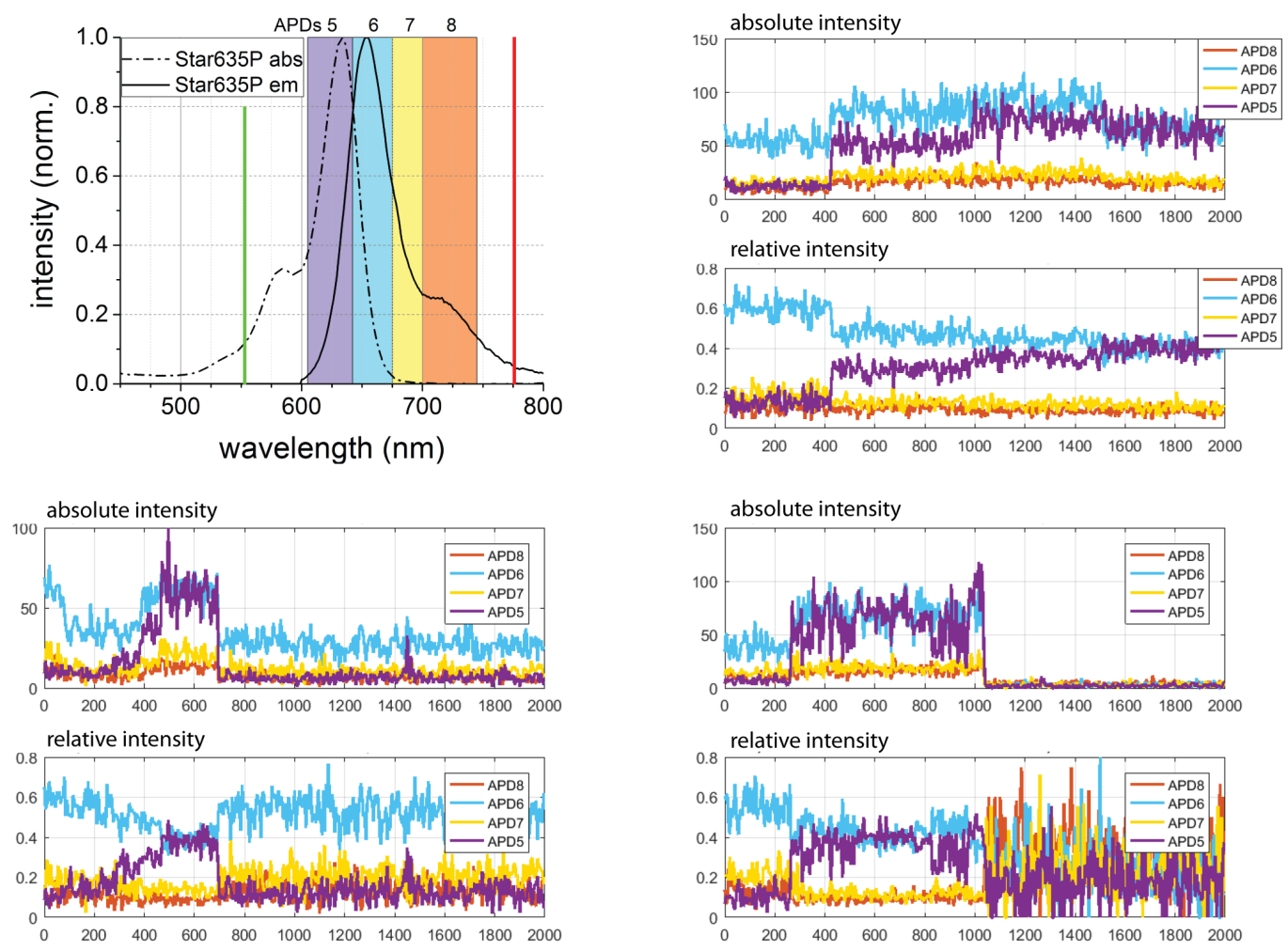

Fig. 21.3: Absorption and emission spectra of Star635P, plotted over the spectral detection windows of APDs 5 to 8 . Excitation at 552nm, STED at $775 \mathrm{~nm}$ not used for these measurements. Besides: Three exemplary fluorescence intensity time traces, each consisting of two plots. The upper plot shows the total photon count per detection channel, the lower plot depicts the relative intensity per detector. The $\mathrm{x}$-axis states the time in $\mathrm{ms}$, corresponding to 400 frames of $5 \mathrm{~ms}$ duration each. 


\begin{tabular}{|l|c|c|c|c|c|}
\hline dye & $\begin{array}{c}\text { no } \\
\text { shift }\end{array}$ & $\begin{array}{c}\text { spectral } \\
\text { shift }\end{array}$ & blue & red & both \\
\hline Atto532 & 103 & 91 & 31 & 41 & 19 \\
\hline Atto532 & $53 \%$ & $47 \%$ & $34 \%$ & $45 \%$ & $21 \%$ \\
\hline Star635P & 85 & 89 & 40 & 16 & 33 \\
\hline Star635P & $49 \%$ & $51 \%$ & $45 \%$ & $18 \%$ & $37 \%$ \\
\hline $\begin{array}{c}\text { Star635P } \\
\text { STED }\end{array}$ & 70 & 109 & 46 & 26 & 37 \\
\hline $\begin{array}{c}\text { Star635P } \\
\text { STED }\end{array}$ & $39 \%$ & $61 \%$ & $42 \%$ & $24 \%$ & $34 \%$ \\
\hline
\end{tabular}

Tab. 21.1: Number of intensity traces of single Atto532 and Star635P molecules that either emit continuously with the same emission spectrum for the duration of the scan or that undergo at least one spectral shift towards the blue, the red or both consecutively. Atto532 was tested for photoconversion only in confocal mode, whereas Star635P was tested both confocally and with 775nm STED. The measurements of Star635P with STED are described in detail in the following section 21.2.

In the first time trace in the top right graph of Fig. 21.3, three blueing steps of a Star635P molecule can be observed, during which the intensity on APD 5 increases while the intensity on APD 6 simultaneously decreases. After the first two steps, the total fluorescence intensity increases, because the photoproducts are excited more efficiently by the 552nm light. In contrast, after the third step, the total intensity decreases, because either absorption coefficient or QY of the photoproduct is reduced.

The second time trace in the bottom left graph of Fig. 21.3 contains four blueing steps of the dye followed by a red-shift. One can identify the blue-shifts at $100 \mathrm{~ms}, 300 \mathrm{~ms}$, 400ms and about 470ms both by the intensity shift from APD 6 to APD 5 in the lower graph and by the different average photon counts on each detector plotted in the top graph. At about 700ms acquisition time, a large red-shift occurs. The resulting molecule is spectrally very similar to the original, but has a markedly lower emission, either due to reduced absorption or QY.

In the third time trace on the bottom right of Fig. 21.3, quick interconversions between different blue-shifted photoproducts can be seen, starting at about 300ms. They are followed by a single photobleaching step.

In total, 297 traces of Atto532 molecules and 261 traces of Star635P molecules were analyzed in this manner. Those that contained fluorescence emission were manually classified into traces which showed spectral shifts and traces which did not. Traces that contain at least one spectral shift were further categorized into ones which show either only shifts towards the blue, only shifts towards the red or both successively. The results in absolute numbers of traces and percentages are stated in table 21.1. 
One can see in table 21.1 that about half of all measured Atto532 and Star635P molecules underwent at least one spectral shift during the observation period. More Atto532 molecules shifted to the red than to the blue, and about $21 \%$ did both. Conversely, the majority of Star635P molecules shifted to the blue, 37\% changed emission in both directions consecutively and just $18 \%$ shifted only to the red.

\section{Scatter plots of mean fractional intensity per trace segment}

All intensity time traces were manually segmented at each time point where the molecule's emission shifted or the molecule bleached, as described above. This resulted in 436 segments of Atto532, corresponding to individual fluorescence emission states, and 371 segments of Star635P emission. The fractional intensity per frame was averaged in each channel over the entire segment, resulting in a four-component vector. Additionally, the total number of frames in the segment was noted. Each segment is represented by one data point in the 3D scatter plots in Fig. 21.4 and Fig. 21.5. The fractional intensities on three detectors are used as coordinates of each data point. The fourth dimension corresponding to the fourth detector can be omitted because the relative distribution values add up to one. In both figures, a 3D view and the three $2 \mathrm{D}$ projections of the same 3D distribution are shown.

Looking at Fig. 21.4 and Fig. 21.5, one can see a quite homogeneous distribution of data points. One can identify the original dye spectrum as the densest cluster of points. From there, the data points spread out mainly into three directions in case of Atto532 and into two main directions in case of Star635P. The lack of separate clusters is somewhat surprising, because a small set of photoconversion products is expected to have a certain discrete set of emission spectra. One reason could be that each photoproduct could experience a small spectral shift due to environmental influences. Another reason is that each intensity trace segment contains a certain amount of background fluorescence counts, which also have a spectral signature. Nevertheless, a k-means clustering was performed on the data set to enable a quantitative description of the magnitude and incidence of spectral shifts. Data points belonging to the same cluster have the same color in the scatter plots. The calculated spectral shifts of each dye are stated in table 21.2 and table 21.3.

In Fig. 21.4, one can see the data points spreading more or less along three lines from the original dye spectrum in clusters 3 and 4 . The first consists of clusters 5,7 , 8 , and 9 and represents a continuous shift to the red. It is especially evident by the anticorrelation of APDs 3 and 4 in the bottom right projection. The second is composed of clusters 6 and 10 and indicates a shift further to the red, which can be seen in table 21.2. The third contains clusters 1 and 2, which consist of blue-shifted photoproducts. This is evident in the top right projection onto APDs 2 and 3. 

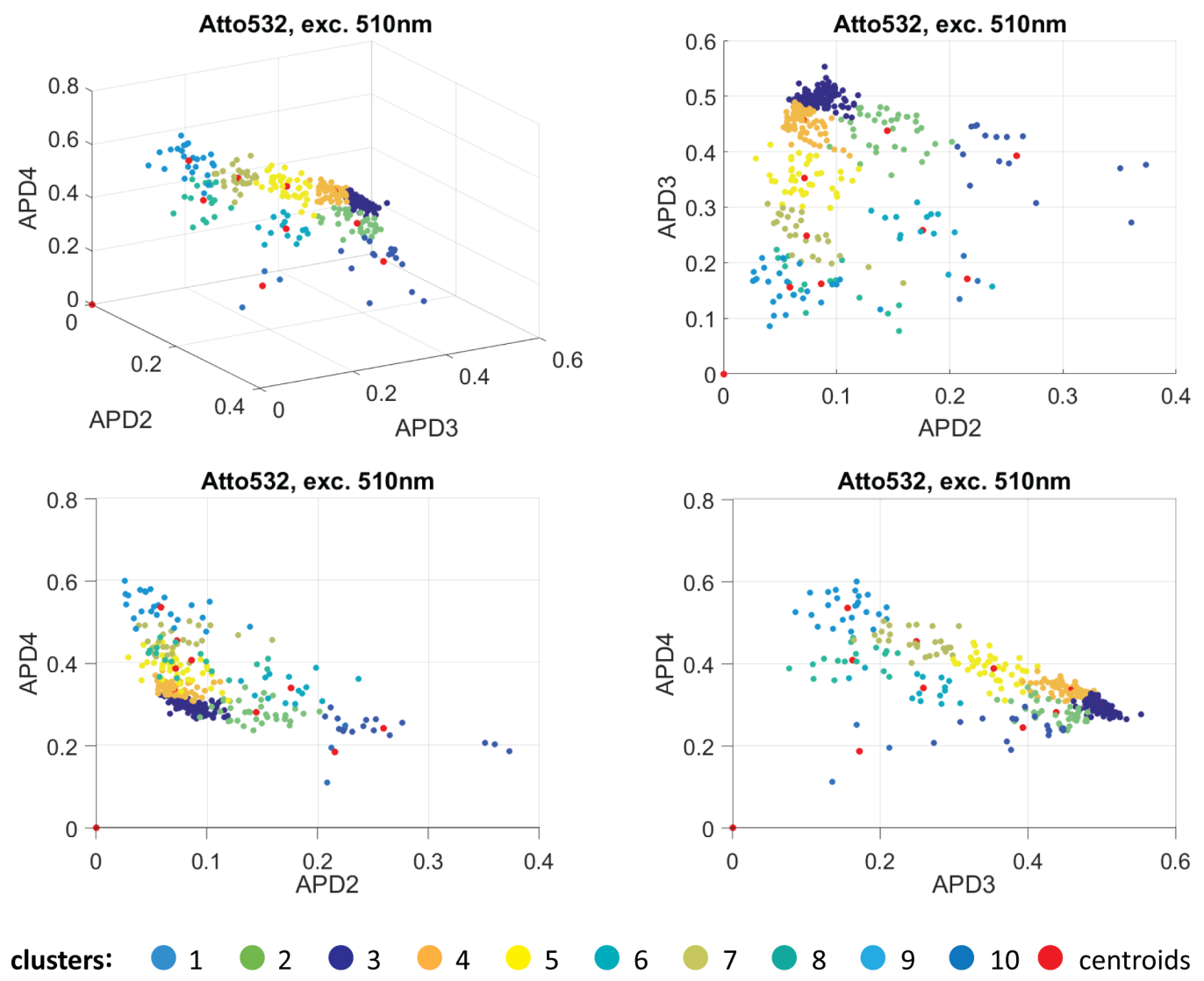

Fig. 21.4: K-means cluster analysis of the spectral intensity distribution of Atto532 (clusters 3 and 4) and its photoconversion products. The averaged fractional fluorescence intensity in each time trace segment is plotted in a 3D scatter plot. A 3D view and $2 \mathrm{D}$ projections are shown. Data points belonging to the same cluster are marked with the same color. The centroid of each cluster is depicted in red.

In Fig. 21.5, one can also see the data points fanning out from the original dye population in cluster 6 . The first branch consists of clusters 1 to 5 , all of which contain blue-shifted photoproducts. This is evident in the top right projection onto APDs 5 and 6 , whose fractional intensities are anticorrelated. The second branch contains clusters 7 to 9 , which feature red-shifted emission. The red-shift is visible as the anticorrelation between APDs 5 and 7 and between APDs 6 and 7 in the two bottom projections.

\section{Cluster analysis of averaged trace segments}

K-means clustering was performed on the data sets of both dyes to estimate the average magnitude and incidence of spectral shifts. The algorithm partitions the data into $\mathrm{k}$ clusters, $\mathrm{k}$ was preset manually. It does so by minimizing the sum of the point-tocentroid distances, which are summed over all clusters. The centroid is the arithmetic mean position of all points in a cluster. Several iterations with new initial values were 

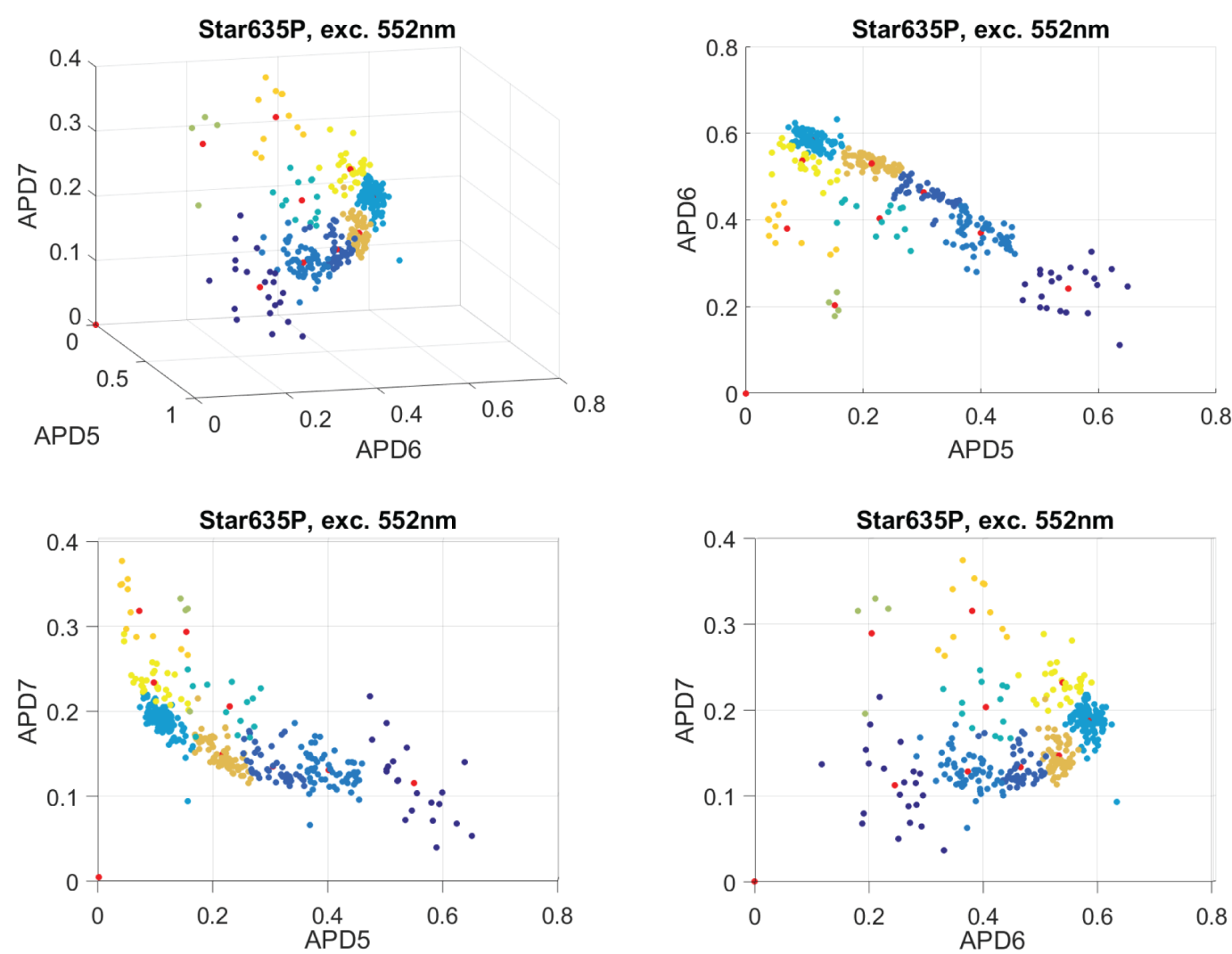
clusters:
$1 \bigcirc 2$
3
4
$5 \bigcirc 6$
$7 \bigcirc 8 \bigcirc 9$
centroids

Fig. 21.5: K-means cluster analysis of the spectral intensity distribution of Star635P (cluster 6) and its photoconversion products. The averaged fractional fluorescence intensity in each time trace segment is plotted in a 3D scatter plot. A 3D view and 2D projections are shown. Data points belonging to the same cluster are marked with the same color. The centroid of each cluster is depicted in red.

\begin{tabular}{|c|c|c|c|c|c|c|c|c|}
\hline $\begin{array}{c}\text { Atto532 } \\
\text { cluster }\end{array}$ & APD2 & APD3 & APD4 & APD5 & shift (nm) & $\begin{array}{c}\text { shift } \\
- \text { offset }\end{array}$ & $\mathbf{n}$ & $\mathbf{t}$ (frames) \\
\hline 1 & 0.26 & 0.39 & 0.24 & 0.11 & 14 & 20 & $16(6 \%)$ & 27 \\
\hline 2 & 0.14 & 0.44 & 0.28 & 0.14 & 8 & 14 & $35(13 \%)$ & 74 \\
\hline 3 & 0.08 & 0.50 & 0.30 & 0.12 & -6 & 0 & 166 & 34 \\
\hline 4 & 0.07 & 0.46 & 0.34 & 0.14 & -10 & -4 & $94(35 \%)$ & 60 \\
\hline 5 & 0.07 & 0.35 & 0.39 & 0.19 & -16 & -10 & $40(15 \%)$ & 32 \\
\hline 6 & 0.18 & 0.26 & 0.34 & 0.23 & -18 & -12 & $14(5 \%)$ & 7 \\
\hline 7 & 0.07 & 0.25 & 0.45 & 0.22 & -21 & -15 & $27(10 \%)$ & 13 \\
\hline 8 & 0.09 & 0.16 & 0.41 & 0.34 & -24 & -18 & $17(6 \%)$ & 13 \\
\hline 9 & 0.06 & 0.16 & 0.54 & 0.25 & -26 & -20 & $24(9 \%)$ & 6 \\
\hline 10 & 0.22 & 0.17 & 0.18 & 0.43 & -53 & -47 & $3(1 \%)$ & 5 \\
\hline
\end{tabular}

Tab. 21.2: Coordinates, i.e. relative intensities on the detectors, of the centroids of Atto532 clusters, therewith calculated spectral shifts, number of data points per cluster and average number of frames of the segments. 
performed to approach the global optimum. In addition to the index of each data point, the algorithm returns the $\mathrm{k}$ cluster centroid coordinates.

The spectral coordinates of each cluster centroid, i.e. the mean fractional intensity per detection channel, are stated in table 21.2 and table 21.3. Additionally, the number of data points $\mathrm{n}$ in each cluster is given. Furthermore, the average number of frames $t$ of all trace segments in each cluster was calculated. It is the time span each fluorescent species was observed. The maximum value possible in this case is 400, which is the total length of the measured traces. All obtained values are significantly below 400, because of photobleaching.

From the fractional intensity per detection channel, the spectral shift of each centroid was calculated. This was done by shifting the emission spectrum of the dye relative to the spectral detection windows, then integrating the emission spectrum over the width of each detection channel, normalizing to one and comparing it to the input distribution until the minimal difference was reached. The MATLAB code is appended in supplementary section 29. With this approach, I assumed that the shape of the emission spectrum remained unchanged and that each detection channel had the same detection efficiency. A small shift of the measured dye spectrum, i.e. the centroid of the largest cluster, relative to the emission spectrum supplied by the manufacturer was calculated. This was subtracted from the other shift values as a constant offset. The reason for this observed spectral shift is likely the modification of the dye with a linker and coupling to the DNA.

In table 21.2, one can see that red-shifted photoproducts of Atto532 are indeed more prevalent than blue-shifted ones, as expected from the statistics in table 21.1. The original Atto532 dye in cluster 3 has a rather short average observation time of 34 frames before it either bleaches completely or photoconverts. The nearest photoproducts in both clusters 2 and 4 are observed almost twice as long. The further the emission shifts to the red, the shorter the survival time of that dye, as one might intuitively expect from large chemical changes.

In table 21.3, blue-shifted photoproducts of Star635P are more common than redshifted ones, also in line with the observations in table 21.1. One can see that the original Star635P dye in cluster 6 is observed almost four times longer than Atto532, it is therefore more stable under confocal illumination. Both nearest photoproducts contained in clusters 5 and 7 have only a slightly longer survival time. The further blue-shifted emission states tend to have shorter observation time.

For both dyes a trend is visible that states with a large emission shift are observed less often and for a shorter period of time. 


\begin{tabular}{|c|c|c|c|c|c|c|c|c|}
\hline $\begin{array}{c}\text { Star635P } \\
\text { cluster }\end{array}$ & APD5 & APD6 & APD7 & APD8 & shift $(\mathbf{n m})$ & $\begin{array}{c}\text { shift } \\
- \text { offset }\end{array}$ & $\mathbf{n}$ & t (frames) \\
\hline 1 & 0.55 & 0.24 & 0.11 & 0.10 & 27 & 31 & $21(9 \%)$ & 10 \\
\hline 2 & 0.40 & 0.37 & 0.13 & 0.10 & 14 & 18 & $56(23 \%)$ & 57 \\
\hline 3 & 0.30 & 0.46 & 0.13 & 0.10 & 8 & 12 & $43(18 \%)$ & 67 \\
\hline 4 & 0.23 & 0.40 & 0.20 & 0.17 & 4 & 8 & $13(5 \%)$ & 19 \\
\hline 5 & 0.21 & 0.53 & 0.15 & 0.11 & 2 & 6 & $64(26 \%)$ & 133 \\
\hline 6 & 0.11 & 0.58 & 0.19 & 0.12 & -4 & 0 & 129 & 124 \\
\hline 7 & 0.10 & 0.54 & 0.23 & 0.13 & -7 & -3 & $30(12 \%)$ & 134 \\
\hline 8 & 0.07 & 0.38 & 0.32 & 0.23 & -19 & -15 & $11(5 \%)$ & 52 \\
\hline 9 & 0.15 & 0.20 & 0.29 & 0.36 & -29 & -25 & $4(2 \%)$ & 66 \\
\hline
\end{tabular}

Tab. 21.3: Coordinates, i.e. relative intensities on the detectors, of the centroids of Star635P clusters, therewith calculated spectral shifts, number of data points per cluster and average number of frames of the segments.

The calculated spectral shifts of up to approximately 30nm indicate chemical changes of the dye molecules, since they are much larger than what would be expected due to environmental influences. These are reviewed in section 21.3.1. However, the exact reaction pathways are unknown, as discussed in section 21.3.2.

\subsection{STED measurements of spectral shifts}

The results detailed in the previous section were obtained with confocal measurements. Additionally, analogous measurements of Star635P molecules were performed with STED illumination in order to judge its influence on the change of emission spectra. The results are described in this section.

It was found previously that Star635P emission shifts mainly towards the blue. It is assumed that the emission of these blue-shifted species deteriorates the resolution in STED images because they are expected to have a lower depletion efficiency at the given STED wavelength. Therefore, the measurements reported here were performed with APDs 4-7 instead of APDs 5-8 to better observe far blue-shifted species. The absorption and emission spectra of Star635P are plotted over the four spectral detection windows in Fig. 21.6, together with the excitation line at 552nm and STED at 775nm.

Single Star635P molecules were immobilized on the surface of a coverslip as depicted in Fig. 16.1. They were located in confocal mode and then imaged with 75mW STED in four spectrally adjacent detection windows over 150 consecutive frames as described in section 14.2. The exact image acquisition parameters are stated in supplementary table 32.2 . 


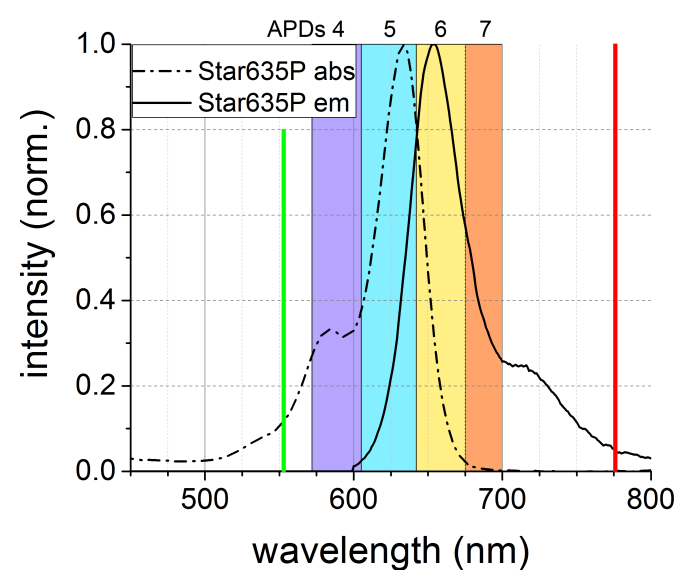

Fig. 21.6: Absorption and emission spectra of Star635P, plotted over the spectral detection windows of APDs 4 to 7 . Excitation at 552nm, STED at 775nm.

\section{Fluorescence intensity time traces}

Time traces showing the absolute photon count in the upper subplot and relative fluorescence intensity on each detector in the lower subplot were plotted over consecutive image frames as described in the previous section 21.1. Examples are displayed in Fig. 21.8 .

In total, 187 such intensity time traces were categorized as detailed previously. Those that contain a spectral shift were classified according to the direction of the shift. The results are stated in table 21.1. One can see that with STED, more spectral shifts are observed than in confocal mode, namely in $61 \%$ of the traces instead of in $51 \%$. However, the direction of the shifts does not change significantly: the majority of molecules shifts only to the blue, $34 \%$ change emission into both directions consecutively and a slightly increased fraction of $24 \%$ shifts emission only to the red.

\section{Scatter plots of mean fractional intensity per trace segment}

The fractional intensity time traces were manually segmented as described previously into sections comprising one emission state of the molecule. In total, 436 sections were selected and analyzed with the MATLAB script appended in supplementary section 29.

The fractional intensity per detector was averaged over each section to obtain one data point with four spectral coordinates. All data points are visualized in the 3D scatter plots in Fig.21.7, which show 3D views of APDs 4-6 and APDs 5-7 and 2D projections of the same data set. Data points that belong to the same cluster according to the k-means 
analysis are marked in the same color in the scatter plots. The centroid of each cluster is marked in red.

Fig. 21.7 cannot be directly compared to Fig. 21.5, because one detector was exchanged, so the intensity fractions per detector are different. The original dye spectrum is contained in cluster 5 , the emission of dyes in clusters 4 and 6 is only slightly shifted. Clusters 1 and 2 comprise far blue-shifted photoproducts, which is evident from the anticorrelation between APD 4 and APDs 5 and 6 in the top right and middle left graph of Fig. 21.7. Cluster 7 consists of a far red-shifted species, which can be seen in the bottom right projection onto APDs 6 and 7. It corresponds to clusters 8 and 9 in Fig. 21.5, as can be seen by comparing tables 21.4 and 21.3. Later, a Gaussian fit of the spatial intensity distribution in each section was performed to estimate the FWHM. Cluster 3 includes all 184 sections where this fit did not converge, which is why the values were set to zero.

\section{Cluster analysis of averaged trace segments}

A k-means cluster analysis was performed on the set of trace segments, as detailed in the previous section 21.1. Since this data set contained fewer data points, the number of clusters was reduced to still enable meaningful averaging. The spectral coordinates of each cluster's centroid, i.e. the mean fractional intensity distribution, were obtained and are stated in table 21.4. In addition to the spectral shift, the number of data points $\mathrm{n}$ per cluster, corresponding to the incidence of the shift, and the average number of frames t per trace segment, representing the survival time of the molecule, were extracted and are also given in table 21.4 .

The spectral coordinates of the centroids were used to calculate the magnitude of their spectral shift. I assumed again that the shape of the emission spectra remained unchanged. Again, changes of the emission to the blue are more common than changes to the red, as in the confocal experiments and in line with the statistics presented in table 21.1. Comparing tables 21.3 and 21.4, one can see that the red shifts calculated for STED clusters 6 and 7 are almost the same as for confocal clusters 7 and 9. The counterpart of confocal cluster 8 was probably split between STED clusters 6 and 7. The incidence of red shifts is higher in case of STED: $38 \%$ of the STED sections compared to $19 \%$ of the confocal sections, or $10 \%$ shifted by $24 \mathrm{~nm}$ in STED versus $7 \%$ shifted by $15 \mathrm{~nm}$ and $25 \mathrm{~nm}$ in confocal mode. The slightly blue shifted STED cluster 4 corresponds quite well to the slightly blue shifted confocal clusters 4 and 5 . All have the emission maximum clearly centered on APD 6. Confocal clusters 2 and 3 do not have a clearly identifiable counterpart in the STED cluster analysis, which may be due to the reduced number of clusters. In both data sets, cluster 1 contains the fluorescent species with the largest blue shift, calculated to be $31 \mathrm{~nm}$ and $61 \mathrm{~nm}$. In both cases, the highest intensity 

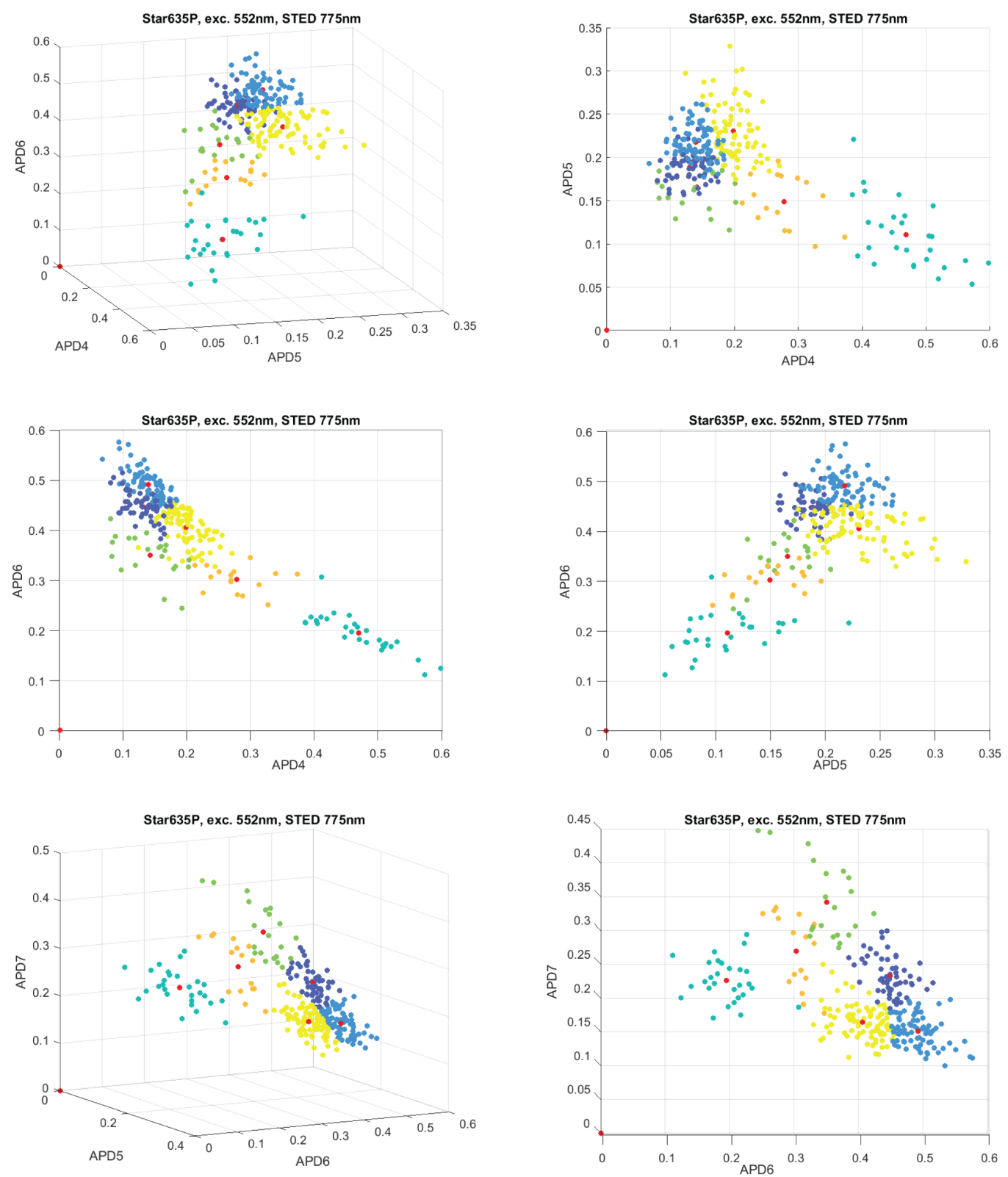

clusters: $1 \quad 2 \bigcirc 3 \quad{ }_{3} \bigcirc 5 \quad{ }_{3} \bigcirc 7 \quad$ centroids

Fig. 21.7: K-means cluster analysis of the spectral intensity distribution of Star635P (cluster 5) and its photoconversion products. The averaged fractional fluorescence intensity in each time trace segment is plotted in a 3D scatter plot. Two 3D views of APDs 4-6 (top) and APDs 5-7 (bottom) and 2D projections are shown. Data points belonging to the same cluster are marked with the same color. The centroid of each cluster is depicted in red. 


\begin{tabular}{|c|c|c|c|c|c|c|c|c|c|c|}
\hline cluster & APD4 & APD5 & APD6 & APD7 & $\begin{array}{c}\text { shift } \\
(\mathbf{n m})\end{array}$ & $\begin{array}{c}\text { shift } \\
-\mathbf{o f f s e t}\end{array}$ & $\mathbf{n}$ & t (frames) & $\begin{array}{c}\text { mean } \\
\text { FWHM (nm) }\end{array}$ & $\begin{array}{c}\text { s.d. } \\
\text { FWHM (nm) }\end{array}$ \\
\hline 1 & 0.47 & 0.11 & 0.19 & 0.23 & 65 & 61 & $28(14 \%)$ & 7 & 119 & 39 \\
\hline 2 & 0.28 & 0.15 & 0.30 & 0.27 & 53 & 49 & $16(8 \%)$ & 9 & 107 & 24 \\
\hline 3 & 0.00 & 0.00 & 0.00 & 0.00 & 49 & 45 & $184(-)$ & 0 & 0 & 0 \\
\hline 4 & 0.20 & 0.23 & 0.40 & 0.17 & 7 & 3 & $81(40 \%)$ & 41 & 86 & 27 \\
\hline 5 & 0.14 & 0.22 & 0.49 & 0.15 & 4 & 0 & $81(-)$ & 35 & 82 & 23 \\
\hline 6 & 0.13 & 0.19 & 0.45 & 0.23 & 2 & -2 & $58(28 \%)$ & 26 & 87 & 21 \\
\hline 7 & 0.14 & 0.17 & 0.35 & 0.34 & -20 & -24 & $21(10 \%)$ & 11 & 87 & 20 \\
\hline
\end{tabular}

Tab. 21.4: Spectral coordinates, i.e. fractional intensities on the detectors, of the centroids of Star635P clusters, and therewith calculated spectral shifts. Additionally, the number of data points $n$ per cluster and average number of frames $t$ of the segments. Further, the average FWHM and s.d. of all sections (all frames added) in each cluster. Cluster 3 contains all scans where the fit of the FWHM did not converge.

is detected on the bluest detector, APD 4 in STED and APD 5 in confocal experiments. So, the emission maximum is not clearly located on one or between two detectors, where its location can be calculated quite precisely. Instead, the right-hand red slope of the emission spectrum needs to be fitted with 4 data points, which is not as accurate. Therefore, this shift value is uncertain, both clusters likely contain the same fluorescent species.

Looking at the average number of frames t per trace segment in each cluster in table 21.4, one can see that photoproducts with a larger spectral shift have a lower survival time before photobleaching or further photoconversion. A similar trend was seen in confocal experiments. Comparing to table 21.3, it seems that the average observation time of the original dye is 3.5 times lower in STED experiments than in confocal. However, the duration of one frame scan was almost four times longer in STED experiments, as stated in supplementary table 32.2. Thus, the observation times of all fluorescent species are comparable in both measurements.

The images in all the frames belonging to a time trace section were summed to obtain a total intensity image. This image was then fitted with a symmetric 2D Gaussian function and the FWHM of the fit was extracted. Examples of raw intensity images and the corresponding Gaussian model functions are depicted in Fig. 21.8, together with the associated intensity time traces.

The average FWHM of all sections in each cluster and the s.d. are stated in table 21.4. If the Gaussian fit did not converge, all values were set to zero, these are contained in cluster 3. One can see that the original dye has the best spatial resolution, with a FWHM of $82 \mathrm{~nm}$ at a STED intensity of $75 \mathrm{~mW}$. The red-shifted fluorophores have a slightly larger FWHM, but not significantly so within the error margin. The far blueshifted fluorescent species in clusters 1 and 2 do have a significantly broader PSF: $25 \mathrm{~nm}$ and $37 \mathrm{~nm}$ more, corresponding to an increase of $30 \%$ and $45 \%$, respectively. This is expected as the blue-shift should decrease the STED efficiency. 

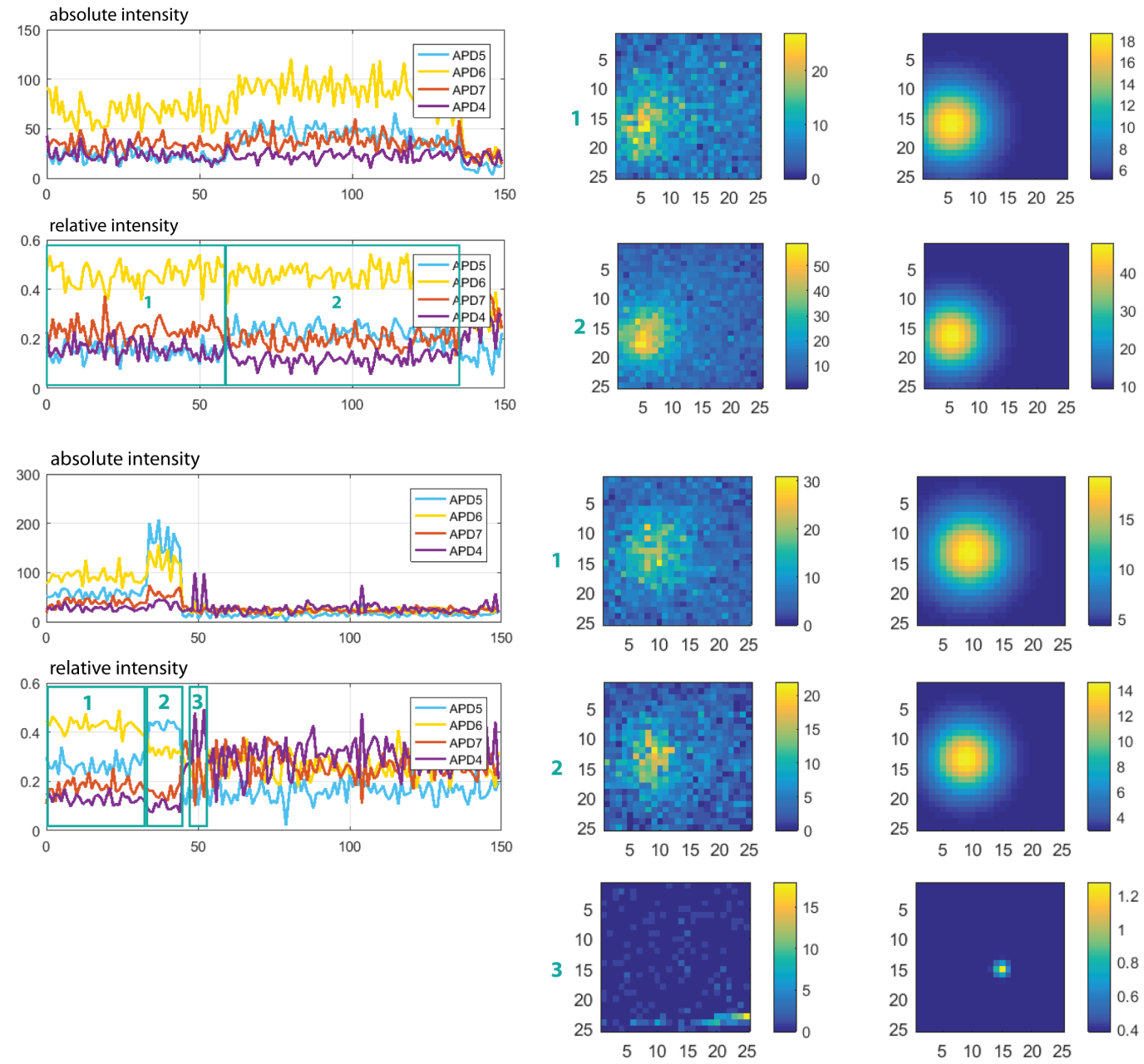

Fig. 21.8: Left: Intensity time traces of absolute photon count (upper subplot) and fractional intensity per detector (lower subplot) are plotted over consecutive frames for two separately scanned regions. The has a ROI size of $25 \mathrm{px}$, which translates to $250 \mathrm{~nm}$. Right: All image frames in the marked sections were added, the total intensity STED image (left) and 2D Gaussian fit model (right) of each section are shown.

The upper intensity time trace in Fig. 21.8 reveals a moderate blue-shift of the emission after approximately 60 frames, which is accompanied by an increase of the absolute photon count in the upper subplot. Accordingly, one can see a higher fluorescence intensity in the second image. However, the Gaussian fit model does not show a marked FWHM increase after blueing, probably due to the counteraction of the increased SNR. In the lower intensity time trace in Fig. 21.8, a larger shift of the emission to the blue is visible after approximately 30 frames, followed by quick photobleaching. Afterwards, a fluorescent contamination diffuses through the observation volume, showing up as a stripe in image three. Comparing images one and two, the FWHM actually decreases slightly due to the increased SNR, instead of increasing due to lower STED efficiency as expected. This behaviour shows one source of the large s.d. of the FWHM stated in table 21.4 . 


\subsection{Summary and discussion}

In this chapter, spectrally and temporally resolved measurements of the fluorescence emission of single Atto532 and Star635P molecules were described. Atto532 was measured in confocal mode and Star635P was measured both in confocal and STED mode. The data provides evidence of spectral shifts of the emission spectrum of individual molecules, both towards shorter and longer wavelengths. The incidence and magnitude of these shifts were analyzed, as well as the observation time period of the shifted species. About half of all molecules undergo at least one change during the observation time. Atto532 shifts more towards the red, whereas Star635P shifts more towards the blue. Species that underwent a larger emission shift tend to have a shorter survival time. The behavior of Star635P is not changed much by STED illumination. Far blue-shifted products of Star635P have a significantly broader FWHM under constant STED illumination. The underlying mechanism of the fluorescence emission shifts was not investigated.

\subsubsection{Spectral diffusion}

Spectral diffusion, which is the change of absorption and emission spectra and spectral width due to changes in the local environment of fluorophores [2], has been reported earlier, see section 3.3. However, the spectral shifts described in the previous sections cannot be due to spectral diffusion by strain, local electric fields, excitation of a matrix or reorientation of matrix molecules, because the experiments were performed in an aqueous solvent at room temperature. Laser-induced heating of the aqueous solvent is expected to be less than $3 \mathrm{~K}$, because the absorption of water is small in the red spectral region ([105], [79]). Even if the sample was heated more, this would be expected to be a continuous process, not to cause a step-wise change. The fluorophore itself is heated during vibrational relaxation after excitation or stimulated emission, which is explained in theory section 2.1. But the fluorophore is cooled within approximately 50ps by collisions with solvent molecules [34], which is about 100 times faster than the fluorophore's excited state lifetime.

Moreover, in contrast to the observations listed in section 3.3, Zhang et al. investigated 14 far-red dyes in immunostained cells and measured a typical s.d. of $2.5-4.5 \mathrm{~nm}$ in the single-molecule spectral mean [149]. They state that "moreover, similar spectra were noted for single molecules labeling different subcellular targets", which might present different microenvironments.

For some molecules, the existence of spectrally distinct isomers is reported ([66], [36]). However, from the structure of the dyes studied here, depicted in Fig. 21.9, one can see 
that they do not exhibit isomerism. In the case of rhodamines, dimer formation leads to a blue-shift according to [112]. Dimer formation as a reason for spectral shifts is excluded here, because single fluorophores were attached to individual DNA strands in my experiments.

One further argument for photoconversion is that the blueing described for ensembles of fluorophores in [142] is irreversible. When I imaged single regions of fixed cells stained with red fluorophores with STED and then examined the same sample again the next day, the scanned regions could be identified by the naked eye in the microscope eyepiece, because they glowed much brighter under green widefield illumination.

Furthermore, the emission changes reported in the previous sections took place on a much faster timescale of a few milliseconds instead of hundreds of milliseconds to minutes. All in all, I assert that what is observed here is not spectral diffusion, but photoconversion.

\subsubsection{Photoconversion}

Photoconversion of fluorophores by light-induced chemical reactions from higher excited or triplet states have previously been described in literature, refer to section 3.7. The chemical reactions causing the spectral changes described previously are unknown so far. Based on the structure of the fluorophores Atto532 and Star635P shown in Fig. 21.9 , one can make an educated guess. The following reactions were proposed by Dr. Alexey N. Butkevich in personal communication:

Two sites of Atto532, encircled in blue in Fig. 21.9, might be deethylated sequentially as numbered. Each deethylation would presumably cause a blue-shift of $10 \mathrm{~nm}$ to $15 \mathrm{~nm}$. This hypothesis could explain the origin of the species in clusters 1 and 2 in table 21.2, which are shifted by $14 \mathrm{~nm}$ and $20 \mathrm{~nm}$. It is unlikely that the sulfonate groups would be attacked and it is unclear what the resulting emission spectrum would look like. It is unknown which reactions might cause the observed red-shifts.

In case of Star635P, several reactions are conceivable. The two rings marked in blue in Fig. 21.9 could open sequentially by addition of water to form long side-chains. The structure of these and other photoproducts are drawn in supplementary Fig. 28.6. Further, the fluorines of Star635P might be replaced by hydroxyl groups by reaction with water. This would likely result in small red-shifts of the emission spectrum. Moreover, it is imaginable that the rings marked in red might react to form double bonds, which would cause a large emission shift to longer wavelengths. It is unlikely that the phosphate groups are attacked. It is unclear what the resulting emission spectrum would look like, it might shift to the red. 

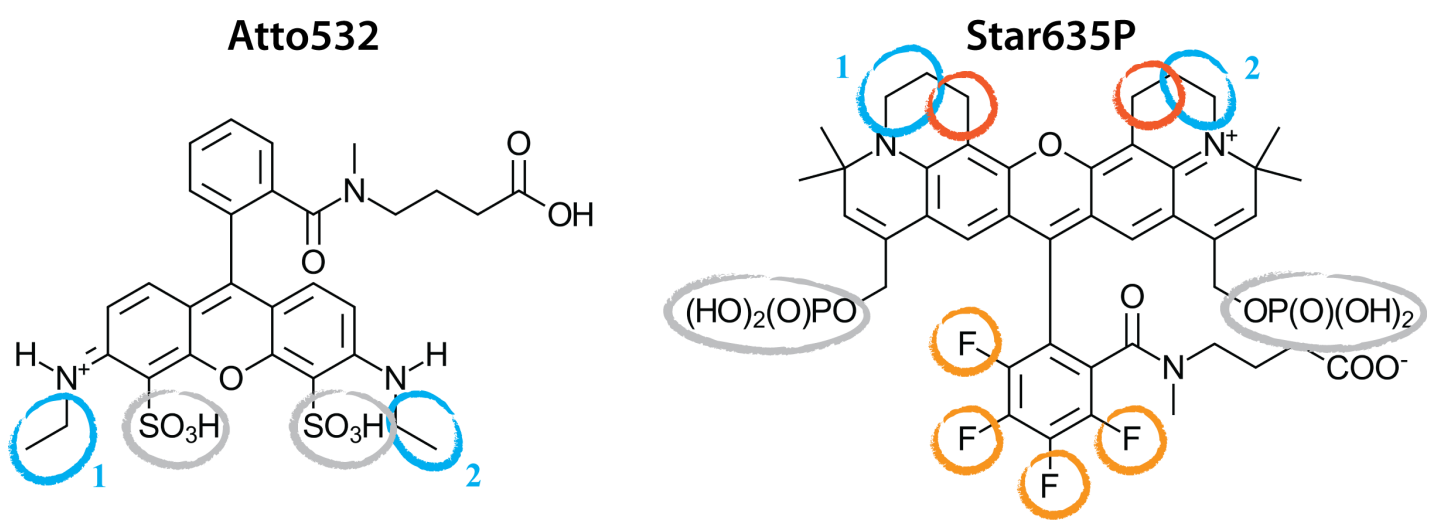

Fig. 21.9: Chemical structure of Atto532, taken from [6], and of Star635P, adapted from [144]. Sites that might be susceptible to photoreactions are marked (suggested by Alexey Butkevich, personal communication, 12th Dec. 2017). Sites whose changes might result in shifts to shorter emission wavelengths are encircled in blue, those that might cause shifts to longer emission wavelengths in orange (small shift) and red (larger shift). Sites that are less likely to be attacked with unknown result are marked in gray. Suggested photoproducts are shown in supplementary Fig. 28.6.

To confirm these hypotheses, the photoproducts would have to be studied further with high-performance liquid chromatography, mass spectrometry or nuclear magnetic resonance, which was out of the scope of this thesis.

\subsubsection{Implications of blueing for STED imaging}

The shift of a fluorophore's emission spectrum towards the blue is in all likelihood accompanied by a similar shift of its absorption spectrum. An indication for this is that often a blue-shift coincided with an increased emission intensity and a red-shift with decreased total intensity. A blue-shift of the absorption spectrum will in turn lead to a lower absorption and therefore lower stimulated emission cross section at the far-red STED wavelength. This will reduce the STED efficiency at a given STED laser intensity and thereby enlarge the FWHM, as seen in table 21.4. Thus, the spatial resolution presumably deteriorates.

One can read from table 21.1 that with STED illumination, $61 \%$ of the measured molecules underwent at least one spectral shift during the observation period. Of these, $42 \%$ shifted towards the blue and $34 \%$ both towards blue and red consecutively, adding up to $76 \%$. Taken together, $46 \%$ of all measured molecules underwent at least one blueing step. In table 21.4 , one can see that of all selected time trace sections, $40 \%$ comprised a small and $22 \%$ a large blue-shift. Since $46 \%$ of the measured molecules transitioned at least once to a blue-shifted state, of which 55\% shifted far, in summary $25 \%$ of all molecules were at least temporarily in a state where their emission can compromise the spatial resolution of STED images. 
However, the molecules were observed in a far blue-shifted state for approximately one quarter of the time period that the original state was observed before further photoconversion or complete photobleaching, as stated in table 21.4.

When a small, countable number of fluorophores is to be imaged, one could scan many short frames analogous to the measurements in the previous section. After acquisition, the spectral distribution could be screened and if a blue-shift is detected, these frames could be discarded before building up the final image. However, one would need to investigate how many fluorophores can be present at most in the observation volume in order to reliably detect individual spectral shifts and to discriminate them from blinking events.

In the graphs in Appendix E, pages 128-129, of [142], one can see that while a fixed cell stained with Star635P is imaged over several frames, the spectral distribution of the measured fluorophore ensemble on the four APDs 5-8 changes continuously towards the blue. Simultaneously, the total fluorescence intensity of the fluorophore population excited with red light decreases while the fluorescence intensity of the fluorophore population excited with green light increases, but with a lower rate. Together, this indicates that a dynamic equilibrium between photoconversion and photobleaching is established. The rate of the spectral change and intensity changes depends on the applied STED intensity. The same behaviour is shown for other red fluorophores. However, the resolution could not be monitored simultaneously because the large nuclear pore complex had been labeled.

Analogous measurements of the fluorescence intensity and spectral distribution of a labeled target structure imaged over several frames could be performed to analyze the dynamic equilibrium of blueing and bleaching rates for a given fluorophore, STED intensity and other scan parameters. Ideally, a small target structure would be chosen which allows the simultaneous measurement of the spatial resolution. Then, an informed choice of scan settings and the number of frames to acquire could be made, which balances total fluorescence signal and blueing in order to optimize the spatial resolution. 


\section{Effect of spectral shifts on the FRET efficiency}

\subsection{Overlap integral changes}

Changes of the emission spectra of single fluorophores were observed in the measurements described in the previous chapter 21.1. In all likelihood, the absorption spectra of the molecules change in a similar fashion. An indication for this is that the total fluorescence intensity changed simultaneously, it often increased upon blueing and decreased upon redding.

The FRET efficiency $E_{\mathrm{FRET}}$ depends on the spectral overlap integral $J(\lambda)$ between the donor emission and acceptor absorption spectra as follows:

$$
\begin{aligned}
J(\lambda) & =\int_{0}^{\infty} \epsilon_{\mathrm{A}}(\lambda) \cdot \lambda^{4} \cdot F_{\mathrm{D}}(\lambda) d \lambda \\
R_{0} & =0.021\left(\frac{\kappa^{2} \cdot Q Y_{\mathrm{D}} \cdot J(\lambda)}{n^{4}}\right)^{1 / 6} \\
E_{\mathrm{FRET}} & =\frac{R_{0}^{6}}{R_{0}^{6}+r^{6}}
\end{aligned}
$$

$J(\lambda)$ is given in units of $\left[\frac{\mathrm{nm}^{4}}{\mathrm{M} \cdot \mathrm{cm}}\right]$ and $R_{0}$ in $[\mathrm{nm}] . F_{\mathrm{D}}(\lambda)$ is the donor emission spectrum normalized to an area of one.

The overlap integral of Atto532 emission and Star635P absorption was calculated with the MATLAB code appended in supplementary section 29. Additionally, it was calculated after the spectra of one or both fluorophores had been shifted numerically

\begin{tabular}{|c|c|c|c|c|c|}
\hline $\begin{array}{c}\text { Atto532 } \\
\text { shift }\end{array}$ & $\begin{array}{c}\text { Star635P } \\
\text { shift }\end{array}$ & $\mathbf{J}\left(\mathbf{n m}^{\mathbf{4}} /(\mathbf{M} \cdot \mathbf{c m})\right)$ & $\mathbf{R}_{\mathbf{0}}(\mathbf{n m})$ & $\mathbf{r}(\mathbf{n m})$ & $\mathbf{E}_{\text {FRET }}$ \\
\hline 0 & 0 & $3.49 \mathrm{E}+15$ & 6.21 & 6.21 & 0.50 \\
\hline 20 & 31 & $3.68 \mathrm{E}+15$ & 6.27 & 6.21 & 0.51 \\
\hline-47 & -25 & $6.70 \mathrm{E}+15$ & 6.92 & 6.21 & 0.66 \\
\hline-47 & 31 & $8.53 \mathrm{E}+15$ & 7.21 & 6.21 & 0.71 \\
\hline 20 & -25 & $1.34 \mathrm{E}+15$ & 5.29 & 6.21 & 0.28 \\
\hline
\end{tabular}

Tab. 22.1: Spectral shifts change FRET efficiency. 
by the experimentally determined maximum and minimum amount stated in table 21.2 and table 21.3. After the overlap integral was computed, the Förster radius $R_{0}$ was calculated. For this, an orientation factor $\kappa=2 / 3$ was assumed and the refractive index $n=1.33$ of water was used. It was further assumed that the shape of absorption and emission spectra remained unchanged and that the donor's QY and acceptor's absorption coefficient staid constant. The FRET efficiency was then computed from $R_{0}$ and a distance of $r=6.21 \mathrm{~nm}$ between the fluorophores. The results are stated in table 22.1 .

The FRET efficiency should be $50 \%$ by choice of $r$, but one can see in table 22.1 that it changes between $28 \%$ and $71 \%$ due to the changes of the overlap integral. Based on the more or less continuous distribution of spectral signatures in the scatter plots in Fig. 21.4 and Fig. 21.5, I expect that theoretically, all values of $E_{\mathrm{FRET}}$ in this range could be observed. Therefore, spectral changes would prevent a quantitative analysis of $E_{\mathrm{FRET}}$ in such single molecule experiments, even in confocal mode. One could only ascertain the presence or absence of FRET.

\subsection{Spectral changes in single molecule FRET time traces}

Single FRET pairs of Atto532 and Star635P attached to dsDNA, which are described in chapter 15, were immobilized on a coverslip surface via streptavidin-biotin interaction as explained in section 16.6. A confocal overview scan of the sample was performed to locate FRET pairs. Then a small region centered on one pair was imaged with FLIMSTED over 50 frames, as detailed in section 14.2. The scan parameters are reported in supplementary table 32.2 . Since two excitation wavelengths were required for ratio imaging, only two detectors could be used, because the software Imspector is limited to four detection channels in total. APD 4 records only donor emission, and the largest fraction of acceptor emission is on APD 6. Excitation was altered in a line-wise fashion to obtain three images quasi simultaneously: the donor channel, i.e. fluorescence excited by 532nm detected on APD4, the acceptor channel, i.e. fluorescence detected on APD6 after 612nm excitation and the FRET channel, which contains fluorescence acquired on APD6 after 532nm excitation. The spectra of Atto532 and Star635P in relation to the spectral detection bands of APDs 4 and 6 as well as the excitation and STED wavelengths are depicted in Fig. 19.1.

This data set, which was acquired with all five FRET constructs for different STED intensities and pulse delays, was used to construct the lifetime histograms presented in chapter 18 and analyzed with regard to the spatial resolution in each detection channel as reported in chapter 19 . 
The raw data of four FRET pairs was analyzed as follows. Fluorescence intensity time traces of the total absolute photon count per detector over consecutive frames were plotted as described previously. Sections of the time trace comprising emission from the same state of the fluorophore were selected manually. For each section, all frames were added to obtain a total fluorescence intensity image. The spatial intensity distribution of each FRET pair was fitted with a 2D Gaussian model function to extract the FWHM. Additionally, the fluorescence lifetime histograms of all pixels in all frames belonging to the section were added to obtain one lifetime histogram with improved SNR. The donor histograms were fitted with a single exponential decay in the software Origin to obtain the lifetimes stated in the figures. Due to the low counts, they were not deconvolved with the IRF. Finally, intensity ratios $E_{\mathrm{FRET}}$ and $R_{\mathrm{FRET}}$ were calculated from the total intensity summed over the entire section in each detection channel. The associated MATLAB code is appended in supplementary section 29. Note that the time traces, lifetime histograms and intensity images of the FRET channel are not corrected for donor bleedthrough and acceptor emission due to direct excitation. The corresponding intensity fractions were subtracted only from the summed photon counts of all frames before the FRET ratios were calculated, as illustrated in supplementary Fig. 28.4. Such sets of data are presented for four exemplary FRET pairs in Fig. 22.1, Fig. 22.2, Fig. 22.3 and Fig. 22.4. In the time traces and lifetime histograms, the donor channel is always marked in green, the acceptor channel in red and the FRET channel in yellow.

The expected FRET efficiency and lifetime of each construct are stated in table 18.1. Construct DNA 79 should have an efficiency of 93\%, corresponding to a donor lifetime of $0.27 \mathrm{~ns}$. In chapter 18 , a lifetime of $0.82 \mathrm{~ns}$ was fitted to the summed histograms of all DNA 79 constructs. The FRET efficiency of construct DNA 80 should be $69 \%$, resulting in a donor lifetime of $1.18 \mathrm{~ns}$. A lifetime of $1.47 \mathrm{~ns}$ was fitted to the summed histograms of DNA 80.

Note that since only two detectors, which are not spectrally adjacent, were used, any spectral changes of donor or acceptor could not be seen directly like in the previous chapter 21. Looking at the emission spectra in Fig. 19.1, one can see that donor blueing leads to an intensity drop on APD 4, whereas acceptor blueing causes an intensity increase on APD 6. Unfortunately any observed intensity changes cannot be related to the magnitude of the spectral shift, because the QY of the dyes and the acceptor absorption coefficient after photoconversion are unknown.

In Fig. 22.1, data of a single FRET construct DNA 80 is presented. In the intensity time traces, one can observe a state change after five frames. First, a high signal is measured in all channels. Then, the photon counts in the donor and FRET channel drop, while the photon count in the acceptor channel remains constant. The remaining signal in the FRET channel stems from directly excited acceptors. Clearly, the donor bleached completely, thus FRET was abolished. The lifetime histograms support this 

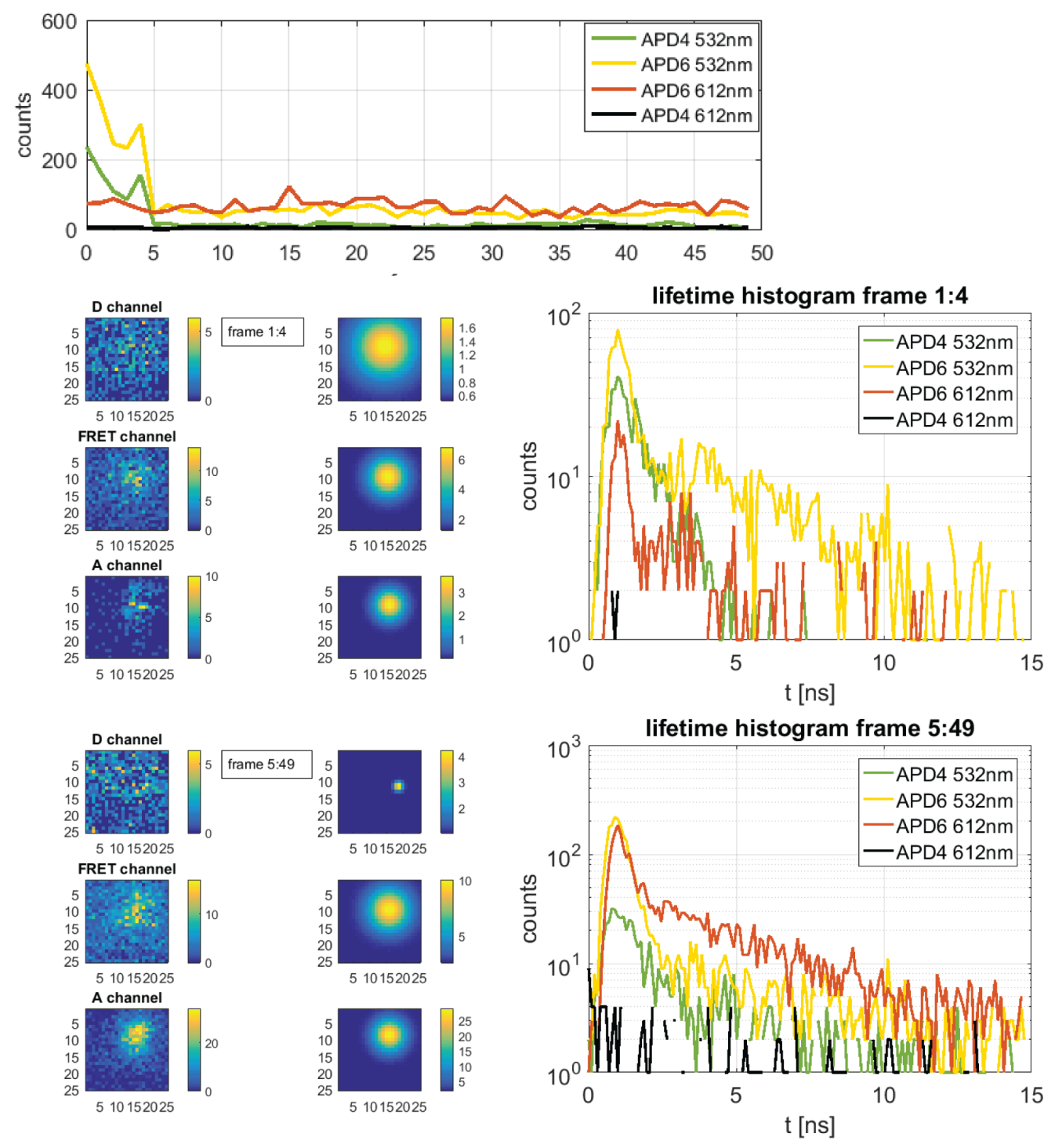

\begin{tabular}{|c|c|c|c|c|c|c|}
\hline frames & FWHM D [nm] & FWHM FRET [nm] & FWHM A [nm] & $\boldsymbol{\tau}_{\mathbf{D}}[\mathbf{n s}]$ & E_FRET & R_FRET \\
\hline $1: 4$ & 150 & 92 & 73 & 1.09 & 0.68 & 4.46 \\
\hline $5: 49$ & 20 & 102 & 77 & 0.62 & 0.81 & 0.76 \\
\hline
\end{tabular}

Fig. 22.1: Data of a DNA 80 FRET construct. Loss of FRET signal due to donor bleaching. Top left: Time trace of photon count in each detection channel over 50 image frames. Middle: Intensity images of 250nm edge length and lifetime histograms summed for each section of the time trace. Bottom: Table of fitted FWHM, fitted donor lifetime and FRET values $E_{\mathrm{FRET}}$ and $R_{\mathrm{FRET}}$. Photon count in the FRET channel is the sum of sensitized emission, donor bleedthrough and acceptor direct excitation. 

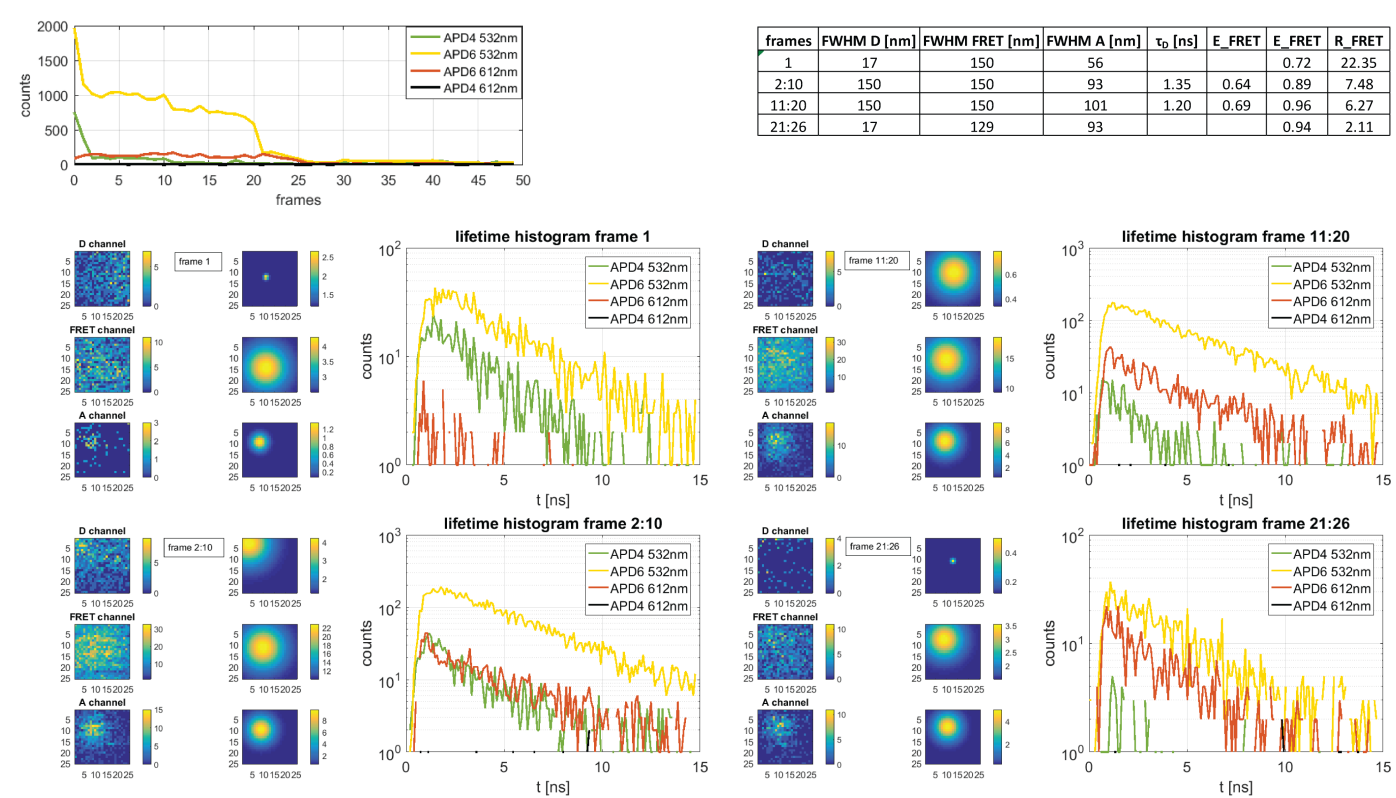

Fig. 22.2: Data of a DNA 79 FRET construct. Donor blueing in two steps reduces FRET signal. Donor bleaches, acceptor bleaches shortly afterwards. Note the changed shape of the last histogram of the FRET channel due to loss of FRET. Top left: Time trace of photon count in each detection channel over 50 image frames. Top right: Table of fitted FWHM, fitted donor lifetime and FRET values $E_{\text {FRET }}$ calculated from lifetime and intensity ratio and $R_{\text {FRET }}$. Below: Intensity images of $250 \mathrm{~nm}$ edge length and lifetime histograms summed for each section of the time trace. Photon count in the FRET channel is the sum of sensitized emission, donor bleedthrough and acceptor direct excitation.

interpretation: in the upper histogram of the FRET channel, a second peak due to energy transfer is visible at about 3ns, which vanished in the lower histogram. The Gaussian fit of the intensity images shows a broader PSF in the FRET channel than in the acceptor channel. This is expected as discussed in chapter 19, but might be aggravated here by a low SNR. Each donor lifetime histogram was fitted with a single exponential decay, the resulting decay times are stated in the table. The fitted donor lifetime of 1.09ns corresponds well to the theoretically expected value of $1.18 \mathrm{~ns}$. It is shorter than the $1.47 \mathrm{~ns} \mathrm{lifetime}$ of the summed histograms. The second donor histogram corresponds to that channel's IRF, which is quite broad at $0.62 \mathrm{~ns}$. The FRET efficiency was calculated from the corrected intensity ratio to be $68 \%$, which also corresponds quite well to the theoretically expected value of $69 \%$. However, the value of $81 \%$ obtained for the second section of the trace is unexpected because it should be close to zero. This means that some correction was inaccurate, probably that of the local background. However, that is difficult to achieve for such a low photon count.

The intensity time trace of a DNA 79 FRET construct in Fig. 22.2 reveals four events: First, the donor presumably undergoes two blueing steps after frames 1 and 10. This is indicated by the simultaneous decrease of the absolute photon counts in donor and FRET channel. Then the donor bleaches completely after frame 20 and the acceptor 
bleaches at frame 27. When comparing the fourth lifetime histogram of the FRET channel with the three previous ones, one can see from the changed shape of the peak that FRET is abolished after donor bleaching. The intensity image of the FRET channel has a quasi confocal resolution, as expected for this FRET construct from chapter 19. The donor lifetime histograms of both long sections were fitted with a single exponential decay, the resulting lifetimes are stated in the table. They are significantly longer than the theoretically expected $0.27 \mathrm{~ns}$ or the $0.82 \mathrm{~ns}$ obtained from fitting the summed lifetime histograms. When assuming an original donor lifetime of $3.81 \mathrm{~ns}$, taken from Fig. 22.3, they result in FRET efficiencies of 64\% and 69\%. These values are lower than expected. They are also lower than those calculated from intensity ratios, again showing difficulties in making spectral corrections at low counts. Based on the intensity trace, two blueing steps were assumed. Both should reduce the FRET efficiency due to decreased spectral overlap. Indeed, both donor lifetimes are longer than expected, meaning $E_{\mathrm{FRET}}$ is lower. As the first value is smaller, the first species should be shifted further to the blue. But the corresponding donor intensity is higher than that in the following time trace section. This can be explained by a change of the QY that accompanied the emission shift. This example highlights the difficulty of disentangling spectral changes from pure changes of the QY, which could have various other reasons as well.

Fig. 22.3 contains data of a single DNA 79 FRET construct. The intensity time trace reveals several events: After the first frame, the donor most likely shifts to the blue, as can be seen from the simultaneous intensity decrease in donor and FRET channel. Between frame 6 and frame 28, the donor transitions to a dark state while the acceptor continues to fluoresce, as can be seen from the disruption of FRET. This disruption is also evident in the changed peak shape of the second FRET lifetime histogram. In frames 29 and 30, the donor shortly blinks on, whereby the FRET signal is restored. The FRET signal is higher than before, which indicates a red-shift of the donor. Between frame 31 and 37, the donor blinks off again, disrupting the energy transfer. In frame 38, the donor turns on again. Presumably it underwent another shift to the red, since the FRET signal is even higher than before. At frame 42, the acceptor bleaches, whereupon the donor emission is unquenched. In the associated lifetime histogram, one can see the significant increase in the donor lifetime to 3.81ns after FRET is abolished. In the last frame, the donor also bleaches. The lifetimes fitted to the donor histograms are again longer than the expected values. However, their step-wise decrease supports the hypothesis of a red-shift, which would increase the FRET efficiency. The observed step-wise increase of the FRET efficiency would be hard to explain in any other way in this sample.

In Fig. 22.4, data of another DNA 80 FRET construct is shown. All donor lifetimes determined from single exponential decay fits are lower than the expected values. This could be due to the contribution of the IRF, which was not deconvolved due to the low 

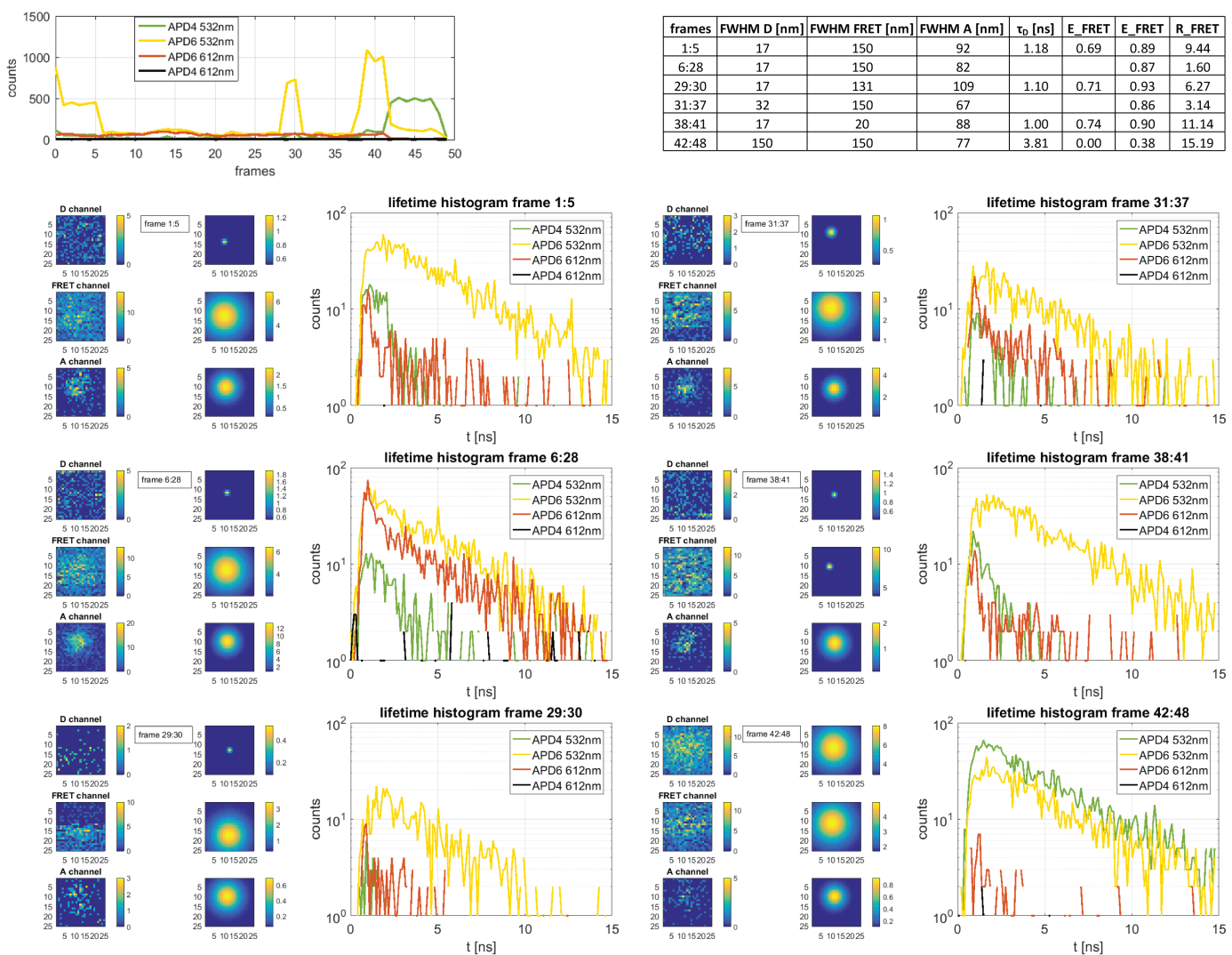

Fig. 22.3: Data of a DNA 79 FRET construct. Donor blueing after first frame reduces FRET signal. Donor blinks off, thus interrupts FRET. Donor blinks on, with increased FRET signal. Donor blinks off again, then on, with yet increased FRET, due to red-shift. Acceptor bleaches, thus unquenches donor emission. Lastly donor bleaches. In the last histogram the increase of the donor lifetime is clearly visible. Top left: Time trace of photon count in each detection channel over 50 image frames. Top right: Table of fitted FWHM, fitted donor lifetime and FRET values $E_{\text {FRET }}$ calculated from lifetime and intensity ratio and $R_{\text {FRET }}$. Below: Intensity images of $250 \mathrm{~nm}$ edge length and lifetime histograms summed for each section of the time trace. Photon count in the FRET channel is the sum of sensitized emission, donor bleedthrough and acceptor direct excitation. 

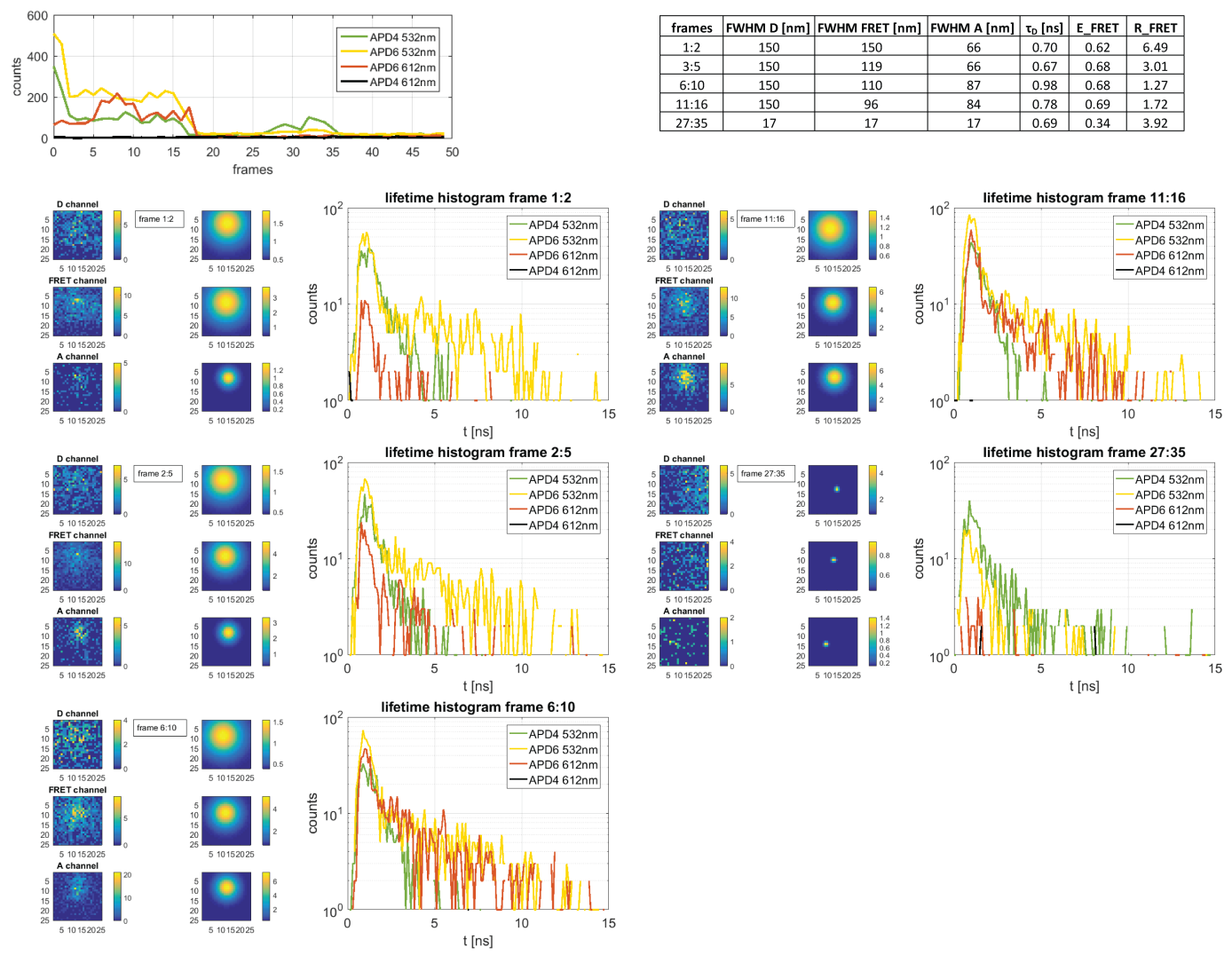

Fig. 22.4: Data of a DNA 80 FRET construct. Donor blueing after second frame lowers FRET. Acceptor blueing, but lower FRET signal in this state. It is probably due to changed absorption, the QY seems similar. Then acceptor redding, FRET signal still constant. Donor bleaches, then acceptor. Later different donor molecule appears. Late photon emission due to FRET clearly visible in histograms. Top left: Time trace of photon count in each detection channel over 50 image frames. Top right: Table of fitted FWHM, fitted donor lifetime and FRET values $E_{\text {FRET }}$ and $R_{\text {FRET }}$. Below: Intensity images of 250nm edge length and lifetime histograms summed for each section of the time trace. Photon count in the FRET channel is the sum of sensitized emission, donor bleedthrough and acceptor direct excitation. 
count rates. Looking at the intensity time trace, the significant drop of donor and FRET intensity after the second frame implies that the donor shifts to the blue. Comparing the shape of the first and second FRET lifetime histogram shows that due to the lower FRET efficiency, less photons arrive at later times. After frame 5, the increased acceptor intensity indicates a shift towards the blue. However, this does not result in an increased FRET signal, as one would expect. Also, the donor lifetime increases significantly, which implies a lower FRET intensity. This is contrary the behavior expected from a blue-shift of the acceptor. It could be that the acceptor's absorption decreased. This shows the difficulty of discriminating between spectral changes and absorption changes, which might also have other reasons. In frame 11, the acceptor signal drops again, but not to the original level. This hints at a blueing shift to an intermediate spectral state, which is supported by an intermediate value of the donor lifetime. However, the total fluorescence intensity in the FRET channel remains approximately constant between frames 3 and 16. This could be due to a changed acceptor QY, as well as a change of absorption proposed above. In frame 17, the donor blinks off and in frame 18 the acceptor bleaches, thus FRET is abolished. From frame 27 to frame 35, the donor appears to switch on again, but with a low emission intensity. That might be due to a blue shift. However, the lifetime is much shorter than that of an unquenched donor should be. This might either mean that it is not an Atto532 molecule, but a fluorescent contamination with a similar spectrum. Or it might be that the acceptor transitioned to a dark state where it retains some absorption but does not fluoresce anymore. 


\subsubsection{Summary and discussion}

In summary, the discussions here show that the correct interpretation of the single molecule FRET traces acquired in two spectral channels is hardly possible once photoconversion is considered. A reliable interpretation would require knowledge of the QY and absorption coefficient of the photoproducts. Even with that information, disentangling spectral changes and changes of QY and absorption would demand further information. On the other hand, the observed changes of the FRET efficiency in such a simple sample would also be hard to explain without taking photoconversion into account. Furthermore, the discrepancy between the FRET efficiency values calculated from fitted donor lifetimes and from intensity ratios highlights the difficulty presented by low photon counts: On the one hand, the lifetime fit is uncertain and additional components of bleedthrough, background, etc. cannot be taken into account by a single exponential fit. On the other hand, the subtraction of local background, donor bleedthrough and directly excited acceptor emission as well as the ratio computation are inaccurate at low counts. For these reasons, a statistical analysis of the whole single FRET pair data set regarding the FRET efficiency was not performed.

In conclusion, for a more reliable FRET efficiency determination more photons would have to be collected without prematurely bleaching the fluorophores. This may be achieved with a different detection design, as was touched upon in chapter 13.1. Yet, splitting the emission into at least two spectrally adjacent detection channels each for donor and acceptor would be necessary to ascertain emission shifts of the fluorophores. 


\section{Part VI}

\section{Further discussions}

This part contains discussions of further topics which are related to the combination of FRET and nanoscopy, but were not investigated experimentally in this thesis.

At the beginning of this part, the determination of donor and acceptor concentrations and their stoichiometry in biological experiments with STED are discussed (chapter 23). Next, homo-FRET as an alternative to hetero-FRET measurements are considered (chapter 24).

Hurdles for uniting FRET with nanoscopy techniques other than STED, both stochastic and coordinate-targeted approaches, are examined (chapter 25). Finally, FRET is compared to the new MINFLUX technique, which also observes single molecules in the sub-10nm range (chapter 26 ). 



\section{Determination of concentration and stoichiometry}

The difficulties of finding a measure for the FRET efficiency which is suitable for STED images were presented in chapter 20. Moreover, it was discussed in chapter 22 that the determination of the FRET efficiency is further hampered if fluorophores photoconvert. Fortunately, in many imaging studies that employ FRET, the exact quantification of the FRET efficiency is not the primary goal. As reviewed in section 7.2, FRET is often used as an indicator of colocalization due to protein interaction or ligand binding, as a reporter of protein activity such as cleavage or phosphorylation, or as a read-out of biosensors.

The FRET efficiency of biosensors in the active/closed state and inactive/open state can be determined beforehand. Then, the fraction of active biosensors in the region of interest is the sought after observable. It can be obtained in several ways, for example by determining the relative contribution of each pattern during a pattern matching analysis, which will be described in the following part VII. Here, analyzing the acceptor lifetime should be sufficient because a biosensor is usually constructed of one donor and one acceptor. Thus, the concentration of active and inactive species could be determined in a subdiffraction observation volume. As a benefit this would reduce spatial averaging of the signal at high concentrations.

If binding interactions, cleavage and such are studied, then the concentrations of free donors and acceptors and bound complexes are wanted. The ratio of free and bound donors can be determined from a biexponential decay fit of the donor lifetime histogram. However, this does not contain diffraction-unlimited information, neither does the bleedthrough into the acceptor channel. The concentration ratio of free and bound acceptors in a subdiffraction volume can be computed for example by a pattern matching analysis.

In biological samples it may happen that several fluorophores are within FRET-range if labeled interaction partners form multimeric complexes. Then, simultaneous energy transfer between all fluorophores is possible and can be analyzed to determine the stoichiometry $v$ of interaction partners in the complex. FRET can be deduced from intensity ratios by two distinct metrics [11]: One measures the fractional reduction of the donor fluorescence intensity. The other reports on the increase of the acceptor 
fluorescence intensity due to FRET. For a 1:1 stoichiometry of donor and acceptor, both metrics yield the same result. If the number of donor and acceptors in a complex is not equal, then their ratio can be determined by comparing both metrics according to [11]:

$$
v=\frac{E_{\mathrm{A}, \max }}{E_{\mathrm{D}, \max }}=\frac{n_{\mathrm{D}}}{n_{\mathrm{A}}}
$$

Here, $E_{\mathrm{A} \text {, max }}$ yields the number of FRET events per acceptor assuming that all donors are excited. Likewise, $E_{\mathrm{D} \text {, max }}$ is the number of FRET events per donor given that all donors are excited.

If hetero-FRET pairs are imaged with STED, then the resolution of donor and acceptor images is not the same, as was discussed previously. Thus the intensity in an image pixel in each detection channel contains contributions from different sample volumes. It follows that in that situation the stoichiometry cannot be determined anymore as described above. This is illustrated in Fig. 20.1. 


\section{Homo-FRET}

In this thesis, so far only hetero-FRET, i.e. FRET between two different fluorophores, was considered. FRET can also occur between fluorophores of the same kind whose absorption and emission spectra overlap due to a small Stokes shift, this is called homo-FRET.

The advantage of homo-FRET is that since only one kind of fluorophore is involved, all fluorophores have the same STED efficiency. Thus one superresolution image could be acquired from which information about FRET could be extracted. However, the FRET efficiency cannot be quantified absolutely. Whether that is strictly necessary depends on the biological application, as discussed in the previous chapter 23.

Since fluorophores of the same kind are involved, the occurrence of homo-FRET cannot be detected with spectral or lifetime measurements. It can only be observed as a decrease of the anisotropy of emitted fluorescence after excitation with linearly polarized light. The decrease of anisotropy occurs as follows: With linearly polarized light, only fluorophores oriented in a certain direction are excited, which is known as excitation photoselection. They emit mainly along the same direction, barring large rotations of the fluorophore during its excited state lifetime. Thus, the fluorescence is highly polarized. The efficiency of FRET depends on the angle between the fluorophore's dipole moments, but FRET occurs also if the dipoles are not parallel. Hence, the acceptor fluorophore can emit at an angle different from the donor dipole and the excitation polarization, which decreases the measured anisotropy. The anisotropy is determined by splitting the fluorescence into perpendicular polarizations onto two detectors.

The disadvantage of anisotropy measurements is that depolarization due to FRET or rotational diffusion have to be discriminated. Furthermore, one has to investigate if fluorophores can rotate freely or if they are restricted by surface interactions and co-rotate with the target protein. If the fluorescence is collected in a time-resolved manner, then depolarisation due to FRET and rotational diffusion can be distinguished, since they usually occur on different time scales. According to the Stokes-Einstein equation, the rotational correlation time constant is

$$
\tau_{\mathrm{DA}}<\tau_{\text {rot }}=\frac{\eta V}{k_{\mathrm{B}} T}
$$


where $\eta$ denotes the viscosity. Since the oligomerization of proteins changes their rotational diffusion, this process can also be observed with time-resolved anisotropy if all monomers are labeled. An example for this application of homo-FRET is reported in [7].

Moreover, when analyzing the anisotropy, its distortion due to scattering by biological medium has to be taken into account, as well as the averaging introduced by high-NA lenses usually used for STED imaging. 


\section{Combining FRET with other nanoscopy techniques}

\subsection{Combining with single molecule localization microscopy}

SMLM requires that only a sparse subset of molecules, spaced further apart than the diffraction limit, is emitting at each point in time. The location of each molecule is determined with high precision as the center of its fluorescence PSF, thus a diffractionunlimited image is acquired. Excitation of a sparse subset is usually achieved by tuning

- on-time and blinking by adjusting the buffer

- photoswitching on-transition rate by adapting the laser intensity

- binding rate by changing the ligand concentration (PAINT).

Extraction of FRET efficiencies from SMLM images has not been experimentally demonstrated yet. The essential pre-condition for doing so is that both donor and acceptor have to be in an excitable state simultaneously for FRET to occur between them. However, whether two neighboring donor and acceptor molecules turn on (or bind, in PAINT) simultaneously and emit enough photons while on is left to chance, because the on-off transitions occur randomly. Therefore, a prolonged acquisition time will be necessary for gathering enough such coincidences to reconstruct an image faithfully with sufficient event density [4]. This practically precludes monitoring of dynamic processes in live cells by FRET using STORM or PAINT. In any case, it would be very hard to determine whether an occurrence or disappearance of FRET was due to changes in the sample or to switching of the labels.

SMLM is conducted in widefield or TIRF microscopy mode. Cameras which can perform TCSPC with picosecond time resolution are currently under development, but the technology is not spread widely yet [52]. Thus, frequency-domain measurements with sinusoidally modulated excitation intensity can be performed to determine the FRET efficiency from lifetime changes. 
An acceptor does not have to emit fluorescence, it only needs to absorb the donor's energy, as a so-called quencher. The FRET efficiency can be deduced from the lifetime of the donor emission. Thus, it could be sufficient to switch only the donor on and off. Acceptor fluorescence emission is strictly necessary only if one wants to determine the FRET efficiency with intensity-based ratiometric methods from two images in separate spectral channels.

Acceptor switching to a non-emitting and non-absorbing state while the donor is still active is yet advantageous, because the brightness of the donor with and without quenching by the acceptor can be compared, yielding additional information about the FRET efficiency. The same information could be accessed by waiting for the acceptor to bleach, as suggested by [56]. Unfortunately, the metastable dark states of fluorescent proteins and fluorophores are often poorly characterized and one molecule can have several of these states. One has to remember that the existence of different nonemitting, but absorbing states could lead to errors in the data analysis and interpretation. Therefore, acceptor fluorescence is useful as an internal control.

PALM uses photoactivatable or photoconvertible labels, which are activated with a certain transition rate and then imaged until they bleach. However, using these irreversible transitions for imaging makes it even more difficult to observe coincidence events during which both donor and neighboring acceptor were switched on, because there is only one chance for this per molecule pair.

The best solution to the challenges described above would be using a photoactivatable donor together with a non-switching acceptor, as pointed out by [40]. Only the donor would need to be activated for FRET to occur and enough signal could be generated before one molecule bleaches ${ }^{1}$. This approach is challenged by the direct acceptor excitation and possibly by ensuing photobleaching by the illumination needed to activate the donor.

The second prerequisite for FRET-SMLM is that a certain number of photons, depending on the SNR, has to be collected during a single molecule event for it to be included in the image analysis. As [40] states: "A few hundreds of photons are sufficient to localize single molecules with a precision that is an order of magnitude better than the resolution of the microscope, but do not allow the accurate detection of multiple FRET states." In practice it will also restrict the range of FRET efficiencies that can be measured during a single molecule burst, because in case of high FRET the donor will emit very few photons and vice versa the acceptor in case of low FRET.

\footnotetext{
${ }^{1}$ The fluorescence emitted per switching cycle is the product of the extinction coefficient, QY and switching-off lifetime of the fluorophore [148].
} 
Super-resolution optical fluctuation imaging (SOFI) is a stochastic nanoscopy method which abandons the prerequisite that only one molecule per diffraction-limited area is allowed to fluoresce, thus enabling fast imaging of many fluorophores emitting simultaneously. Diffraction-unlimited resolution is obtained from higher order statistical analysis of temporal fluorescence intensity fluctuations. Since many molecules are allowed to emit at the same time, it would be more likely in this case that both donor and acceptor are active. If one FRET partner blinks, this induces anticorrelated intensity fluctuations in donor and FRET channel. Neighboring FRET pairs can be distinguished if their emissions fluctuate independently of each other. It follows that in case of a constant FRET signal or in case of concerted fluctuations due to biological processes, the resolution could not be increased. And again, one would have to somehow discriminate between fluctuations caused by photophysical or by biological processes. 


\subsection{Combining with coordinate-targeted nanoscopy other than STED}

The most important factor with regard to combining coordinate-targeted nanoscopy modalities with FRET is whether the state transitions that give rise to the resolution enhancement distort the fluorescence decay from the excited state or not. In both GSD and RESOLFT microscopy, a subset of molecules is transferred to a non-fluorescent metastable state before the image is acquired. The other molecules are not influenced by the switching beam and are afterwards excited to emit a fluorescence signal. Therefore, FRET can occur unaffectedly between the active molecules which are observed at present.

One could switch both the donor and acceptor molecules in order to obtain a diffraction unlimited image. However, it should be sufficient to switch only the donor molecules on and off while permanently leaving the acceptors in the active state. This would be easier to implement because fewer laser lines may be needed. Furthermore, one could optimize the switching parameters of the donor only.

The FRET efficiency could then be determined by spectral and/or lifetime measurements, both are possible with GSD and RESOLFT in point-scanning or parallelized widefield mode. When reversible photoswitching is to be used in RESOLFT, it would be good to use a molecule with uncoupled on- and off-switching, such as Dreiklang [17], as a donor in combination with a non-switchable acceptor. This way, the integration time could be freely adjusted to collect the desired amount of photons.

An additional advantage when shelving part of the donor molecules in long-lived dark states is that a short delay between the switching and the readout pulse can be introduced in order to let any directly excited acceptor fluorescence decay. This would result in a cleaner signal for spectral FRET analysis. In STED microscopy, on the other hand, stimulated emission has to drive the molecules to the ground state immediately after excitation in order to achieve diffraction unlimited resolution. This means that emission from directly excited acceptors in the center of the PSF cannot be avoided. 


\section{Comparison of FRET and MINFLUX}

The localization analysis of MINFLUX relies on the assumption that only one molecule in the probing range is emitting. If several molecules were emitting simultaneously, an averaged position would be obtained, unless the true number of molecules was taken into account. That is why MINFLUX needs photoswitchable, photoactivatable or blinking labels. Therefore, if one wants to use MINFLUX for observing several molecules of the same type located within the probing range, one has to switch them on one by one and localize them sequentially. If this challenge is met, however, one could monitor multiple labeled interaction partners, which is very difficult with FRET due to spectral crosstalk. For this purpose, one could use fluorophores with different emission spectra that could nevertheless be excited simultaneously and localize them by MINFLUX in different detection channels, barring spectral bleedthrough.

Biosensors for small molecules and ions are constantly being developed, as discussed in section 7.2. They consist of a protein binding the molecule of interest with at least two fluorescently labeled subunits. The sensing ability is based on FRET efficiency changes upon conformational changes which are induced by ligand binding [54]. It remains to be seen if such small distance changes between both states can be read out faster and with sufficient precision using MINFLUX.

FRET can measure the relative distance between two dye molecules in a range between $2 \mathrm{~nm}$ and $8 \mathrm{~nm}$ [94]. From this distance and changes thereof, biological information is derived. The precision of the distance measurement is highest around the Förster radius $R_{0}$, which is usually between $4 \mathrm{~nm}$ and $6 \mathrm{~nm}$. It deteriorates for very large and very small distances, because of the $r^{-6}$ dependence of the FRET efficiency. In contrast, the probing range of MINFLUX can be up to circa 150nm in diameter for a single localization step. The localization precision decreases only slightly towards the edge of this range. Therefore, MINFLUX could be used to monitor interaction partners over a longer range, before and after they engage, or to observe for example components of big protein complexes or lipid nanodomains with sizes beyond the dynamic range of FRET.

The FRET rate is proportional to the squared value of the angle between the fluorophore transition dipole moments. Usually, the approximation $\kappa^{2}=2 / 3$ is used, which is based 
on the assumption that the molecules are free to rotate independently and randomly during the observation period [127]. Whether this is the case can be checked for example with anisotropy decay measurements. It turns out that this assumption is indeed not always valid. For example, organic dyes can interact with the surface of the protein they are attached to, causing them to remain fixed in a certain orientation. This can be observed as a co-rotation of the dye with the protein in fluorescence correlation spectroscopy (FCS) measurements [81]. On the contrary, MINFLUX localization measurements are independent of the fluorophore orientation. For excitation a doughnut shaped focus of a circularly polarized beam can be used. Outside its center, the focal field also contains electric field components along the optical axis due to the high NA. Therefore, the molecule can always be excited regardless of its orientation in the focus. Of course, the excitation rate depends on the orientation of the molecule in the local electric field. Therefore, if a molecule was rotating slowly during the repositioning of the doughnut focus, it would be excited differently in the new beam position than in the previous one. Consequently, the fluorescence would also be emitted into a certain direction, causing changes in the detection efficiency. Taken together, this could lead to a localization error, because the localization is done by comparing the fluorescence intensity for different beam positions. However, the rotational correlation time of freely rotating organic dye molecules is less than $10 \mathrm{~ns}$ and the rotational correlation time of entire proteins is on the order of $20 \mathrm{~ns}$ to $100 \mathrm{~ns}$, whereas the exposure time during MINFLUX is several $\mu s$ for each beam position. Therefore, any fluorescence intensity change due to rotation would be averaged out, thereby reducing the localization error.

The fast translational diffusion of proteins and the limited photon emission rate of fluorophores together present a big challenge for ascertaining colocalization with MINFLUX. For example, the maximum emission rate of fluorescein is about 200 photons/ $\mu \mathrm{s}$ [123]. The maximum emission rate of EGFP is 2.9 photons/ $\mu$ s [47] and the brighter fluorescent protein dsRed emits 18 photons/ $\mu$ s at most. As fluorescence is emitted isotropically, a 1.4 NA objective can collect only up to $30 \%$ of the photons due to its limited solid angle. So there will be at most a few tens of photons available per $\mu \mathrm{s}$ to locate a single fluorophore if it was maximally excited. Conversely, the working principle of MINFLUX is to excite the fluorophore as little as possible. On the other hand, the translational diffusion of proteins is very fast compared to the exposure time of MINFLUX. For example, the diffusion coefficient of EGFP in the cytoplasm is $87 \mu \mathrm{m}^{2} / \mathrm{s}$ and that of the enzyme myokinase is $160 \mu \mathrm{m}^{2} / \mathrm{s}$ [3]. So to locate EGFP within an area of $5 \mathrm{~nm}$ diameter, one could take only about $1 \mu \mathrm{s}$ and to localize it within a $0.5 \mathrm{~nm}$ range, one has a measurement period of approximately 10ns. ${ }^{1}$ In conclusion, upon collecting enough photons to precisely locate a single labeled protein, this protein will diffuse within a relatively large volume, thus making it difficult to ascertain colocalization, i.e.

\footnotetext{
${ }^{1}$ Protein association rates vary widely from $10^{3}-10^{9} \frac{1}{\mathrm{M} \cdot \mathrm{s}}[106]$.
} 
protein interaction, within that time period. In contrast, FRET operates reliably, as it reports the donor-acceptor distance independently of the diffusion of the complex.

When large and live biological samples such as tissue sections or live embryos are to be monitored, for example with light sheet microscopy, it is disputable if MINFLUX, even if implemented in a parallelized, multicolor mode, will replace FRET as a reporter of colocalization or as a biosensor readout. 



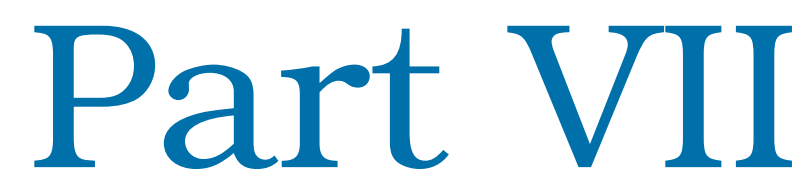

Conclusions and outlook

The physical phenomenon of FRET has been established as a valuable research tool in cell biology. In combination with live-cell fluorescence microscopy, it can be used to gather functional information

- by reporting on colocalization due to protein-protein interactions, oligomerization, or binding of ligands;

- by measuring protein activity, e.g. cleavage or phosphorylation of substrates;

- by reading out FRET biosensors. 
Instead of using FRET, the first two application classes could in principle also be studied with high-resolution multicolor STED imaging, which could directly visualize the location of interaction partners. However, certain classes of FRET biosensors could not be replaced by direct STED imaging because they report on things that cannot be labeled with fluorophores, such as small metabolites, ions, molecular oxygen, mechanical tension or molecular crowding. Applications of such FRET biosensors could benefit from the improved spatial resolution afforded by STED, if on average more than one biosensor is present in a confocal detection volume. The spatial averaging of the signal within the detection volume could be significantly reduced by a higher spatial resolution, which would in turn increase the dynamic range of the biosensor readout. Furthermore, if FRET is used to study processes that occur in adjacent subdiffraction volumes such as vesicles, mitochondrial compartments or small membrane patches, these could be discriminated with STED.

Despite this potential for functional imaging of intricate biological processes on subdiffraction scales, information about FRET has to date not been extracted from superresolved images. To the best of my knowledge, FRET has so far only been used to complement nanoscopy or to facilitate nanoscopy techniques. Thus, the aim of this thesis was to investigate the feasibility of STED imaging of FRET pairs, i.e. to extract information about the FRET efficiency from diffraction unlimited images.

One prerequisite for FRET is that the donor emission and acceptor absorption spectra overlap. It follows that for hetero-FRET, the acceptor emits at longer wavelengths than the donor. With conventional fluorophores, it is better to choose a STED wavelength suitable for the acceptor, i.e. red-shifted from the acceptor emission. A STED wavelength suitable for the donor would efficiently excite the acceptor and the large required STED intensity would easily lead to extreme acceptor bleaching. Since the donor absorbs and emits at shorter wavelengths than the acceptor, it is consequently depleted less efficiently by the far-red STED wavelength. Thus, the image formed by donor emission has a lower spatial resolution than the acceptor image. Therefore, the FRET efficiency should rather be quantified from the acceptor emission.

The FRET efficiency can be calculated from the fluorescence intensity, lifetime and/or anisotropy. Anisotropy-based methods are not sensitive enough for quantitative FRET determination. The different spatial resolution of the donor and acceptor images prevents the computation of the FRET efficiency from intensity ratios for three reasons:

- because the intensity of an image pixel in each channel contains contributions from a different sample volume.

- because the intensity ratio is distorted by the different depletion efficiency of donor and acceptor. 
- because the varying spatial resolution causes artifacts during pixel-wise ratio calculation.

As shown in this thesis, with STED nanoscopy the FRET efficiency can be quantified more reliably based on the fluorescence decay curve of the acceptor.

Numerical simulations explored the interference of STED with the FRET process and illustrated the effects of changing the STED intensity and pulse delay. They comprised the ground and first excited singlet states of a FRET pair and electronic transitions between these due to absorption, spontaneous and stimulated emission and FRET. A comparison of fluorescence decay curves measured and simulated for different illumination parameters showed that this simple model is sufficient to understand the interplay of these photophysical processes.

The shapes of the decay curves of different FRET pairs under pulsed STED illumination were shown to be distinct. Thus, in principle the varying FRET efficiency should be deducible. However, simulations revealed that the spatial resolution is degraded if the STED pulse is shorter than the duration of the energy transfer, which is dictated by the lifetime of the donor. Several approaches can alleviate this issue:

- Simulations demonstrated that a donor with a sufficiently short lifetime could prevent the resolution degradation. Unfortunately, a short fluorescence lifetime often correlates with a low QY, which in turn restricts the Förster radius and the overall fluorescence intensity.

- Time gating was discussed and tried. If the lifetime of the acceptor is much longer than that of the donor, the STED pulse could be delayed until most donors decayed and then the acceptor image could be constructed from photons emitted after the STED pulse. Due to the rather short lifetimes of organic fluorophores to date, this approach would drop an overly large fraction of fluorescence and thereby reduce the SNR.

- A time gate could be used to select only photons emitted during or shortly after the STED pulse to construct an acceptor image with optimal resolution. But for this, enough photons need to be collected from the acceptor in that time period, otherwise the resolution will be deteriorated by a low SNR.

- Long STED pulses or CW-STED could be used to increase the spatial resolution in the acceptor image regardless of FRET efficiency and donor lifetime.

Simulations indicated that with CW-STED, pulsed excitation, and time gating an improved spatial resolution in the acceptor image should be easier to achieve. Simulations of FRET pairs with different transfer efficiency under CW-STED showed that the shapes of their lifetime histograms differ such that the FRET efficiency could in principle be 
elucidated. In practice the limitations set by low photon counts and a high background are to be investigated.

During this thesis work, I upgraded the microscope setup built by Franziska Winter and me. I updated the measurement acquisition software to enable TCSPC for lifetime measurements and implemented automated measurements via the new Python interface. Without the Python scripting, it would not have been possible to gather the single molecule STED data sets presented here. For further large-scale acquisition of single molecule data, the implementation of a focus lock and a computer-guided sample stage would be useful.

For the selection of organic dyes as a STED-compatible FRET pair, several factors turned out to be important:

- photobleaching rate;

- photoconversion behavior;

- excitation of the donor by the STED beam via two-photon absorption; and

- excitation of the acceptor by the STED beam.

I found that the excitation of several dyes by the given STED wavelength was negligible, so a good pair can in all likelihood be selected. If that should not be the case, one would have to include an additional scan step during which only the STED beam illuminates the sample. The signal gathered during this step could then be subtracted from the normal images as illustrated in supplementary Fig. 28.4. However, this step would induce additional bleaching of the dyes.

In single molecule measurements, photoconversion of individual dyes was observed. Photoconversion of the fluorophores is particularly undesirable in FRET-STED. On the one hand, a shift of the acceptor's emission and absorption towards shorter wavelengths reduces the STED efficiency and thus can deteriorate the spatial resolution of the image. On the other hand, a shift of the donor emission or acceptor absorption spectra changes the spectral overlap integral and hence the FRET efficiency. Therefore, photoconversion has to be avoided by choice of appropriate dyes and scan parameters, if possible. Otherwise, it has to be closely monitored to facilitate an accurate FRET efficiency calculation. As it was shown that in the case of Star635P the incidence and magnitude of photoconversion is not significantly altered by STED, this presents a more general problem.

This thesis showed that imaging of FRET pairs with STED nanoscopy is not straightforward. It was discussed how other coordinate-targeted nanoscopy approaches, namely GSD and RESOLFT, may be better suited for diffraction-unlimited FRET studies. The reason is that the state transitions exploited for overcoming the diffraction limit occur 
before signal acquisition, thus they do not interfere with the FRET process during imaging. SMLM techniques are not ideally suited for obtaining superresolved information about FRET either. Stochastic off-switching of one or both interacting fluorophores hinders the observation of FRET and its correct analysis.

\section{Future directions of research}

\section{Acceptor pattern matching}

In order to quantify the FRET efficiency from the acceptor decay curve, a pattern matching analysis seems advantageous because the complexity of the pattern does not influence the complexity of the analysis. Thus the analysis can easily incorporate the following contributions to the FRET channel decay curve:

- sensitized acceptor emission of one or more FRET species;

- directly excited acceptor emission with $\tau_{\mathrm{A}}$;

- bleedthrough emission of paired donors with $\tau_{\mathrm{DA}}$; and

- bleedthrough emission of unpaired donors with $\tau_{\mathrm{D}}$.

An excellent description of pattern matching applied to FLIM is given in [41]. The authors compared the performance of their algorithm to other linear decomposition methods, namely maximum likelihood estimation, a nonnegative least squares algorithm, and phasor analysis. They found pattern matching to be fast and accurate. Moreover, in contrast to phasor plot analysis, spectral information can easily be included and thus more than three patterns can be distinguished simultaneously, which was demonstrated by Niehörster et al. [89]. They discriminated nine fluorophores in a single experiment and also successfully applied their analysis to unmix two-color STED images.

Additionally, pattern matching needs only comparatively few photons for a reliable analysis, in contrast to other methods. Gregor et al. [41] demonstrate the separation of three fluorescent species with a relative s.d. of less than $10 \%$ using 1000 photons per pixel. Niehörster et al. [89] state in supplementary note 1 that they can distinguish five patterns using 4000 photons with a relative s.d. of about $4 \%$. In contrast, Chen et al. [23] use 20,000 photons per pixel for their phasor plot analysis with acceptor lifetime ingrowth.

In order to employ pattern matching, knowledge of the underlying nature of the decay is not necessary, but well-defined and distinct reference patterns with high SNR are required, i.e. normalized probability density functions that describe how many photons 
to expect per time interval [41]. These patterns can either be extracted from the data itself in several ways as detailed in [41] and [89], or measured from samples labeled with a single fluorophore or single FRET species of known efficiency.

Typically, one would like to know the relative fraction of interacting and free donors and acceptors, as discussed in chapter 23. Resolving the individual contributions to the pattern is analogous to spectral unmixing. A suitable algorithm is presented in [41] and a fast variant in supplementary note 3 of [89].

Further research would have to evaluate the accuracy and precision of the pattern matching regarding the following parameters, as it was done in [41], [89], or [63]

- bias towards any and variance of each fraction;

- low fraction of one species;

- low photon counts;

- high fluorescence background; and

- similarity of the reference patterns.

\section{Implementing CW-STED}

When using pulsed STED, it was seen in simulations in section 11.5 and shown experimentally in chapter 19 that the FWHM of individual FRET pairs broadens if the STED pulse is shorter than the FRET process. One way to circumvent this and achieve a high spatial resolution would be to use CW-STED in combination with pulsed excitation and time gated detection. The corresponding fluorescence decay curves were simulated in chapter 10. The shape of the acceptor decay curve varies for different FRET pairs, therefore the determination of the FRET efficiency or the unmixing of different contributing FRET pairs should in principle be possible.

CW-STED might cause less photobleaching as reported by Oracz et al. [91]. This would hopefully allow the collection of more FRET signal. Whether CW-STED also reduces the incidence of photoconversion needs to be investigated as well.

\section{Moving to widefield imaging}

Often, FRET is used to observe processes such as signal transduction that occur across a whole cell or between cells. Imaging such large volumes, preferrably in 3D, is slow with single point-scanning STED. Fortunately, STED has been massively parallelized by Fabian Bergermann [12] to image an area of $33 \mu \mathrm{m}$ edge length within four seconds while maintaining a spatial resolution of below $70 \mathrm{~nm}$ in cells. One positive side effect 
is that photobleaching is reduced because the STED power is shared more efficiently between neighboring minima.

As discussed previously, for information about FRET the acceptor fluorescence lifetime has to be analyzed when using STED. Cameras with TCSPC capability are being developed, with which widefield FLIM could be performed. Alternatively, one might perform frequency-domain measurements with modulated excitation intensity. It is however questionable if such frequency data of the acceptor could be analyzed to unmix all the different contributions listed previously, as this would require a high signal.

\section{Donor STED with non-fluorescent acceptors}

An alternative FRET approach could circumvent both the problems presented by the differing spatial resolution and the difficult quantification of $E_{\text {FRET }}$ from the sensitized acceptor emission. One could use non-fluorescent quenchers as acceptors and perform STED imaging of the donor. The quenched donor lifetime $\tau_{\mathrm{DA}}$ and thus $E_{\mathrm{FRET}}$ could then be deduced directly from the superresolved image by established exponential tail fitting methods.

However, one would first have to test how the quencher behaves under STED illumination. Although it does not fluoresce, it could absorb the STED light. Thus, the quencher might be saturated, which would inhibit FRET. Or it might blink or bleach, both would disrupt FRET. A suitable approach for testing non-fluorescent quenchers as acceptors was described in [72]. Le Reste et al. placed a quencher, which has a very broad absorption spectrum, between a green and a red donor on dsDNA. By monitoring the emission of both donors, they could sort out quencher dependent and independent photophysical events and could characterize quencher blinking and bleaching. Exemplary spectra of Star580 and Star635P, both well suited for STED with 775nm light, paired as donors with the quencher Atto612Q are illustrated in supplementary Fig. 28.7.

Additionally, one would have to determine the minimal number of photons and minimal SNR that are required for the determination of $E_{\mathrm{FRET}}$ with a desired precision and accuracy. STED reduces the number of photons by suppressing the emission of most fluorophores around a target structure. In addition, the more efficient FRET, the more the donor fluorescence is quenched. The combination of both effects could restrict the maximal observable FRET efficiency. 


\section{RESOLFT nanoscopy of FRET pairs}

In RESOLFT nanoscopy, diffraction-unlimited imaging is achieved by reversibly switching molecules to a non-fluorescent state in a coordinate-targeted manner. The transfer of a subset of molecules to the non-fluorescent state occurs before the fluorescence image is acquired. Afterwards, the molecules which were not influenced by the switching beam are excited to emit a fluorescence signal. Due to this uncoupling between the state transition giving rise to the resolution enhancement and the fluorescence excitation and emission, RESOLFT is in principle well suited for imaging FRET. FRET could occur unaffectedly between donor and acceptor molecules when both are in the fluorescent state in the center of the observed volume.

One could think of switching both the donor and acceptor molecules in order to obtain a diffraction unlimited image. However, it should be sufficient to switch only the donor molecules on and off while permanently leaving the acceptor in the active state. This would also be easier to implement because fewer laser lines are needed. The FRET efficiency could be determined when the donor is fluorescent, by spectral and/or lifetime measurements. RESOLFT-FLIM measurements have already been demonstrated by Testa et al. [119]. An additional advantage of shelving donor molecules in long-lived dark states is that a short delay between switching and readout pulses can be introduced in order to let any directly excited acceptor fluorescence decay. This would provide a cleaner signal for FRET analysis.

In order to determine the FRET efficiency precisely, a sufficient number of photons in each pixel is needed, approximately 1000 for FLIM-FRET. A sufficiently long on-time of the fluorophores could be achieved by using molecules with decoupled on- and off-switching mechanisms. In this case, the donor molecule could be switched on, then excited to emit or transfer to the acceptor an adequate number of photons, and afterwards switched off by another wavelength. The fluorescent protein Dreiklang [17] offers these characteristics. Since fluorescent proteins switch slowly compared to STED, a rather long dwell time per pixel would be needed. This can lead to unacceptably long image acquisition times in a point scanning RESOLFT mode. Fortunately, RESOLFT has already been implemented in a parallelized fashion, permitting image acquisition in a $120 \mu \mathrm{m} \times 100 \mu \mathrm{m}$ field of view in less than one second [24].

FRET has been observed between many different pairs of fluorescent proteins. Among the successfully used pairs are EYFP $(513,527 \mathrm{~nm}) \& \operatorname{rsTagRFP}(567,585 \mathrm{~nm})$ and mVenus $(515,528 \mathrm{~nm}) \& \mathrm{mKO} \kappa(551,563 \mathrm{~nm})$ [8]. The reversibly photoswitchable protein Dreiklang absorbs at $412 \mathrm{~nm}$ and $511 \mathrm{~nm}$ in the on-state and at $340 \mathrm{~nm}$ in the off-state; its emission maximum is at $529 \mathrm{~nm}$. Thus, Dreiklang is spectrally very similar to mVenus and EYFP and should therefore exhibit efficient FRET when paired with 
TagRFP or mKO $\kappa$. One should keep in mind that TagRFP is a weak dimer and mKO $\kappa$ is a monomer, which is preferable as an acceptor.

\section{Monitoring photoconversion}

It was shown that photoconversion of fluorophores can degrade the STED image resolution and hampers the FRET analysis. Therefore, it would be beneficial to systematically study the dependence of fluorophore photoconversion on excitation intensity and wavelength, on STED parameters, and buffer composition. Thereby, image acquisition parameters could be optimized to prevent photoconversion as much as possible. Furthermore, robust 'non-converting' fluorophores might be designed, manufactured and studied. 

Part VIII

Supplementary Information 



\section{Supplementary theory}

\section{7}

\subsection{Theory of acousto-optical modulators}

An acousto-optical modulator (AOM) contains a transparent crystal to which an acoustic wave with wavelength $\lambda_{\mathrm{a}}$ is applied, which creates a periodic modulation of the index of refraction. This acts like a phase grating to diffract incident light of the appropriate wavelength $\lambda_{0}$. According to the Bragg condition, the separation angle depends on the acoustic and optic wavelengths as

$$
\frac{\lambda_{0}}{\lambda_{\mathrm{a}}}=\sin \theta_{\mathrm{i}}+\sin \theta_{\mathrm{d}}
$$

with the angles of incident and first order diffracted light $\theta_{\mathrm{i}}$ and $\theta_{\mathrm{d}}$. The intensity of the diffracted light is proportional to the power of the applied acoustic wave and the length of the crystal, i.e. the interaction length of light and acoustic wave. Further information can be found e.g. in [58].

\subsection{Theory of acousto-optical tunable filters}

At the heart of an acousto-optical tunable filter (AOTF) is a birefingent uniaxial crystal. A radio frequency is applied to the crystal with a piezo element to periodically modulate the index of refraction. This acts like a phase grating, so that a portion of the incident light with the matching wavelength is diffracted, as illustrated in Fig. 27.2. The amplitude of the radio frequency regulates the relative intensity of diffracted and undiffracted light.

Under phase-matching condition the wavevectors of the incident beam $\vec{k}_{\mathrm{i}}$, the diffracted beam $\vec{k}_{\mathrm{d}}$ and of the acoustic wave $\vec{k}_{\mathrm{a}}$ are related by

$$
\vec{k}_{\mathrm{i}}=\vec{k}_{\mathrm{a}}+\vec{k}_{\mathrm{d}}
$$

This is illustrated in the wavevector diagram in Fig. 27.3, where o denotes the ordinary polarization and e the extraordinary polarization in the anisotropic uniaxial crystal. 


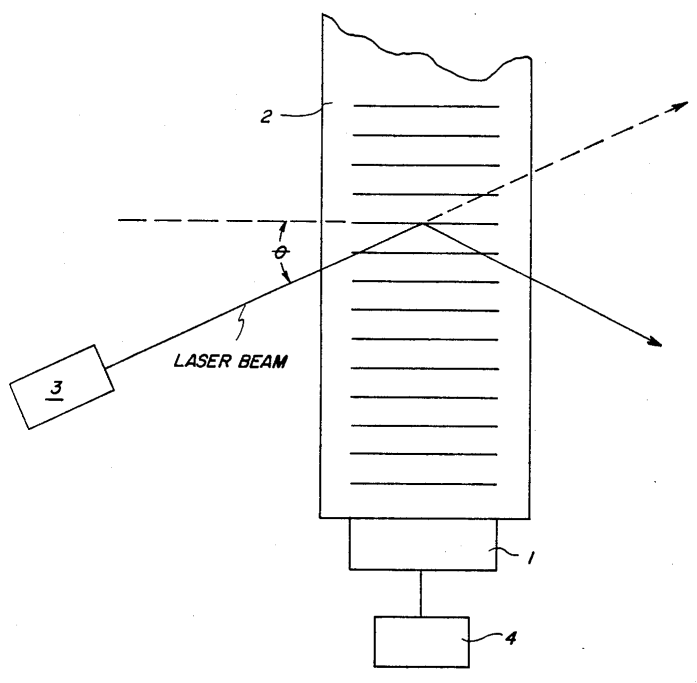

Fig. 27.1: Schematic illustration of a laser beam being diffracted by an acoustic wave inside an AOM crystal. Taken from [15].

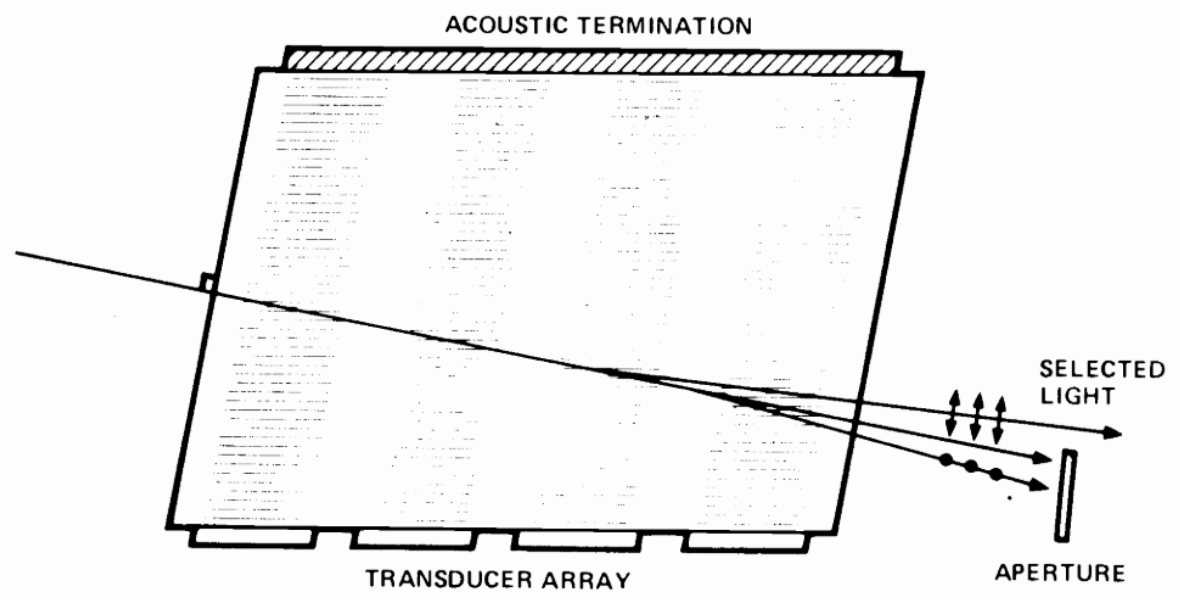

Fig. 27.2: Schematic of the acoustic diffraction of incident light in an AOTF. Taken from [21]. 


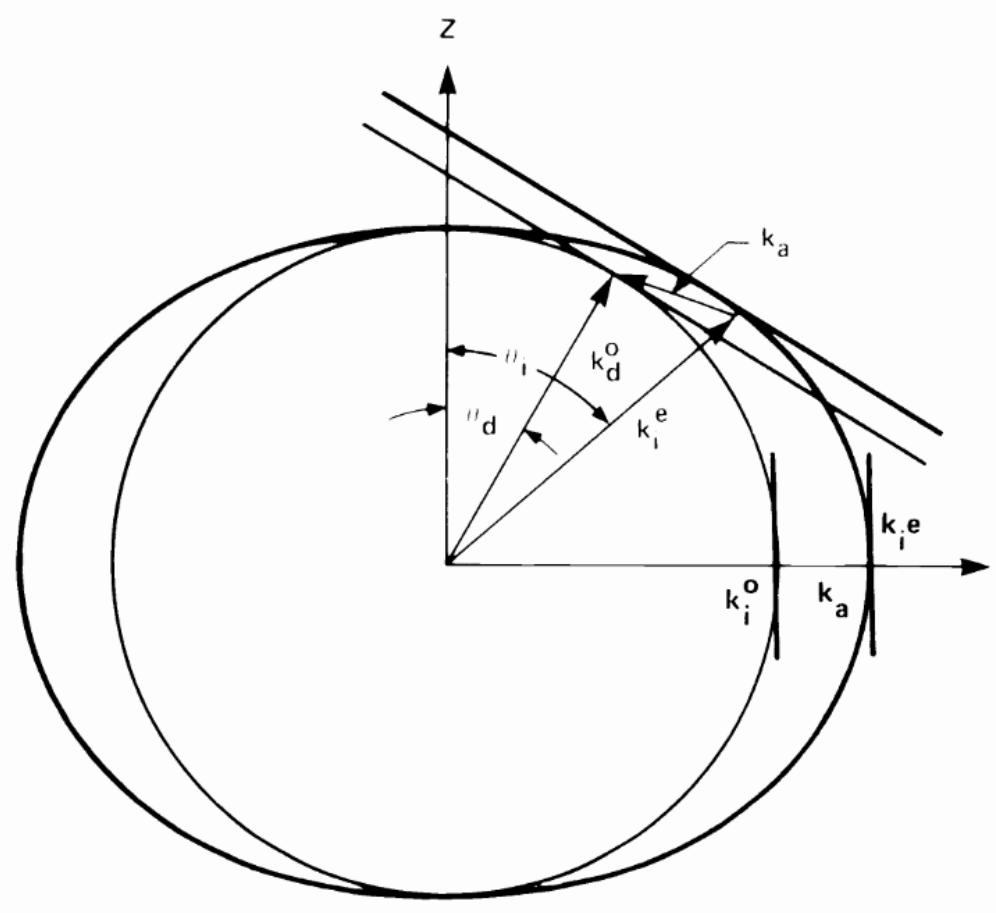

Fig. 27.3: Wavevector diagram for acousto-optic diffraction in a uniaxial crystal. Taken from [21].

According to [21], the approximate tuning relation of an AOTF for an incidence angle $\theta_{\mathrm{i}}$ of $90^{\circ}$ is derived as

$$
f_{\mathrm{a}}=\frac{V_{\mathrm{a}} \cdot \Delta n}{\lambda_{0}}
$$

with the acoustic frequency $f_{\mathrm{a}}$, the acoustic velocity $V_{\mathrm{a}}$, the optical wavelength $\lambda_{0}$ in vacuum and the birefringence $\Delta n=\left|n_{\mathrm{e}}-n_{\mathrm{o}}\right|$.

The intensity of the diffracted beam $I_{\mathrm{d}}$ increases with the applied acoustic power density $P_{\mathrm{d}}$ and the interaction length $L$ of light and acoustic wave as

$$
\frac{I_{\mathrm{d}}}{I_{\mathrm{i}}}=\sin ^{2}\left(\frac{\pi^{2} M_{2} P_{\mathrm{d}} L^{2}}{2 \lambda_{0}^{2}}\right)^{(1 / 2)}
$$

with the acousto-optical figure of merit $M_{2}$. 

Supplementary figures 

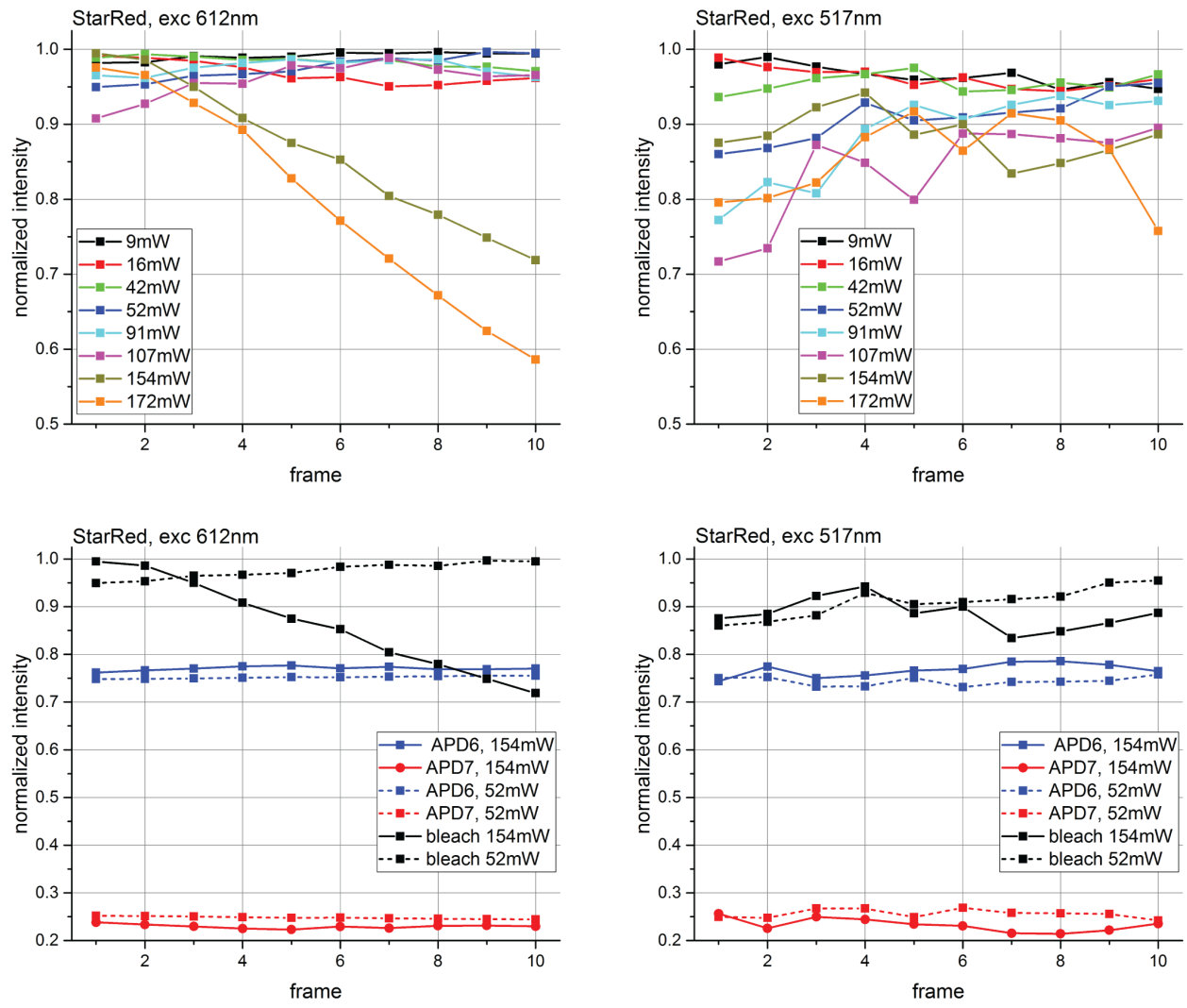

Fig. 28.1: Top: Normalized fluorescence intensity of Star635 over consecutive frames. Fluorescence increase due to destruction of non-fluorescent dye aggregates by bleaching, then fluorescence decrease visible. Bottom: Normalized ratio of intensity detected on APDs 6 (blue) and 7 (red) remains largely constant, showing that spectral shifting was low. Intensity detected on APDs 3 and 4 was negligible. 

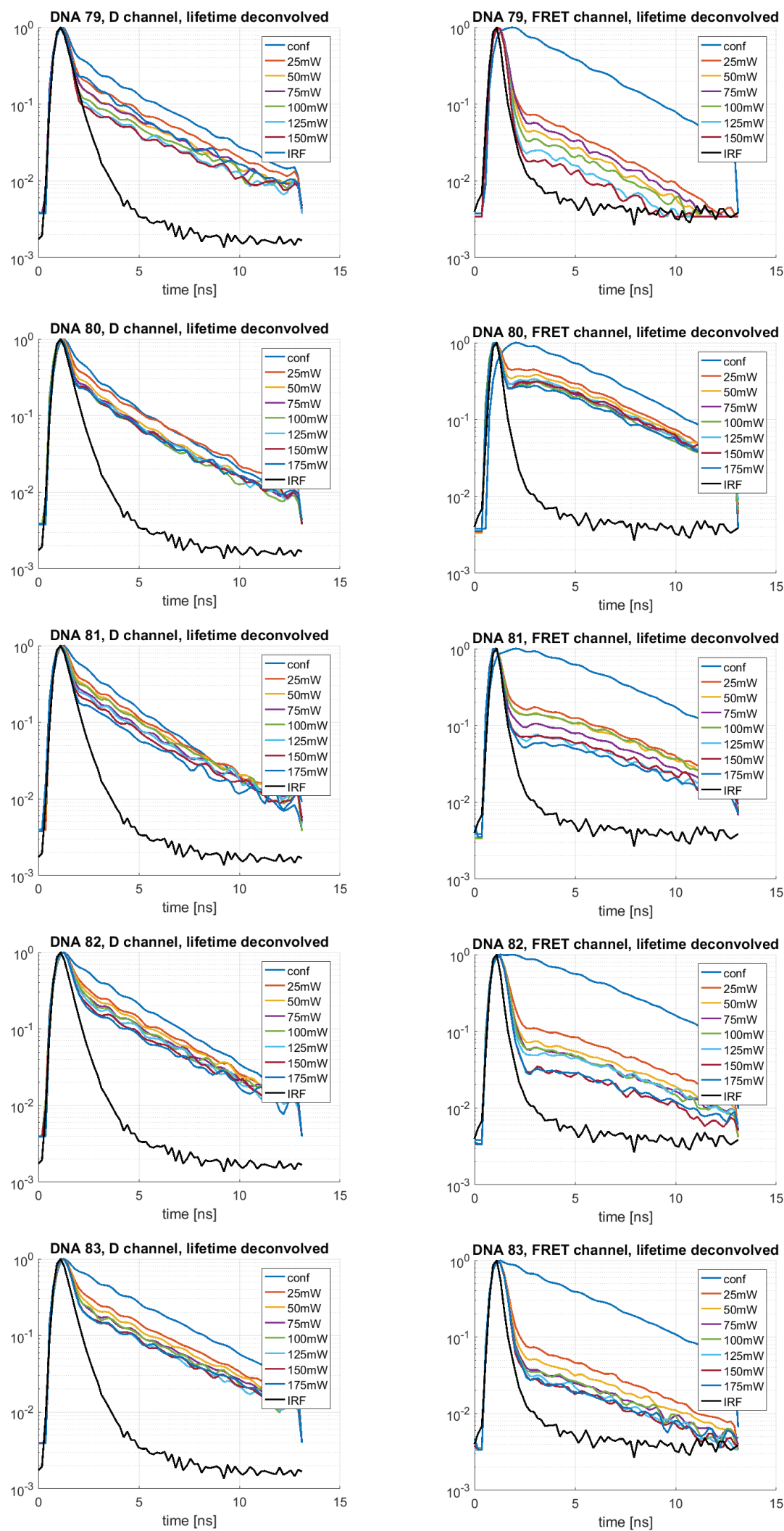

Fig. 28.2: Lifetime histograms accumulated in donor and FRET channel for each single molecule FRET construct with different STED intensity while keeping the pulse delay constant. 

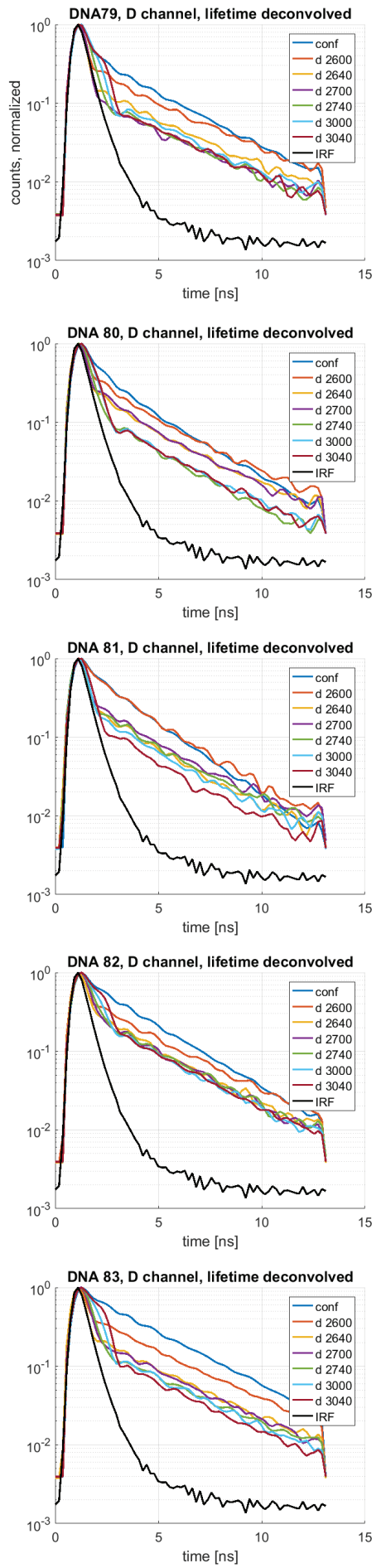
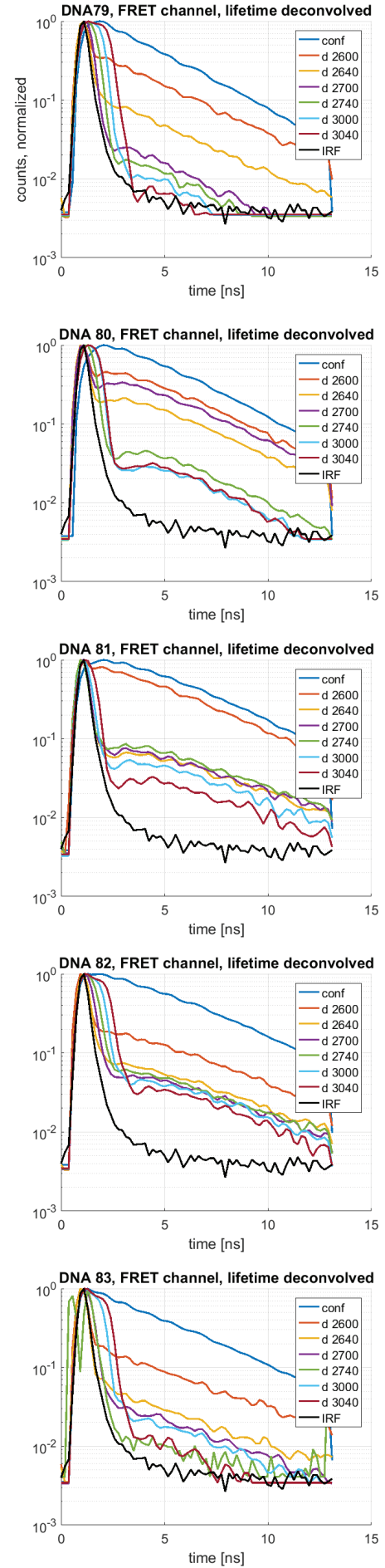

Fig. 28.3: Lifetime histograms accumulated in donor and FRET channel for each single molecule FRET construct. The STED pulse delay was increased in 320ps steps while keeping the STED intensity constant. The legend states the delay value in a.u., a conversion to ns is given in table 14.1. The origin of the double peak in the lowest right figure is unclear, because the corresponding curve in the donor channel, which was acquired simultaneously, looks normal. 


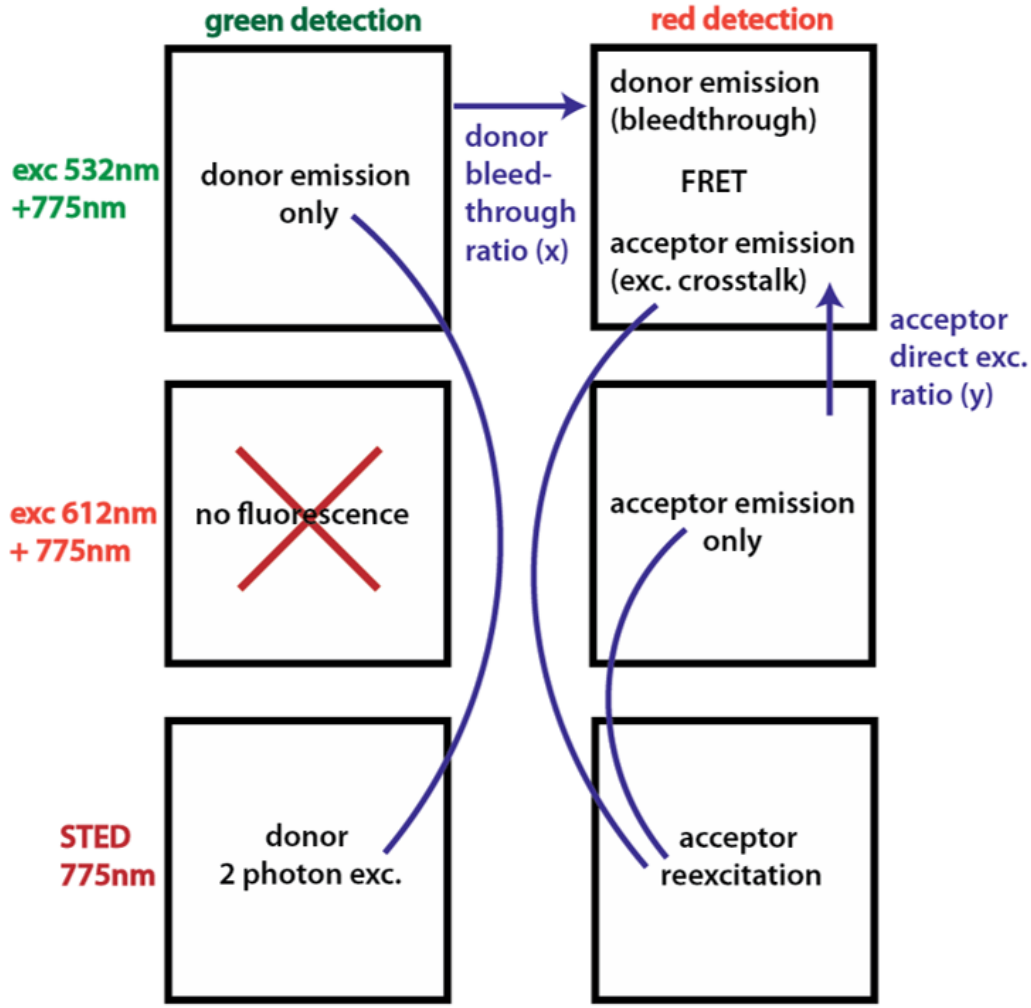

Fig. 28.4: Scheme of spectral corrections of the raw image in the FRET channel. Correction for excitation by the STED beam can be neglected if the dyes are chosen well. The correction factors $\mathrm{x}$ and $\mathrm{y}$ are determined from singly labeled reference samples. 

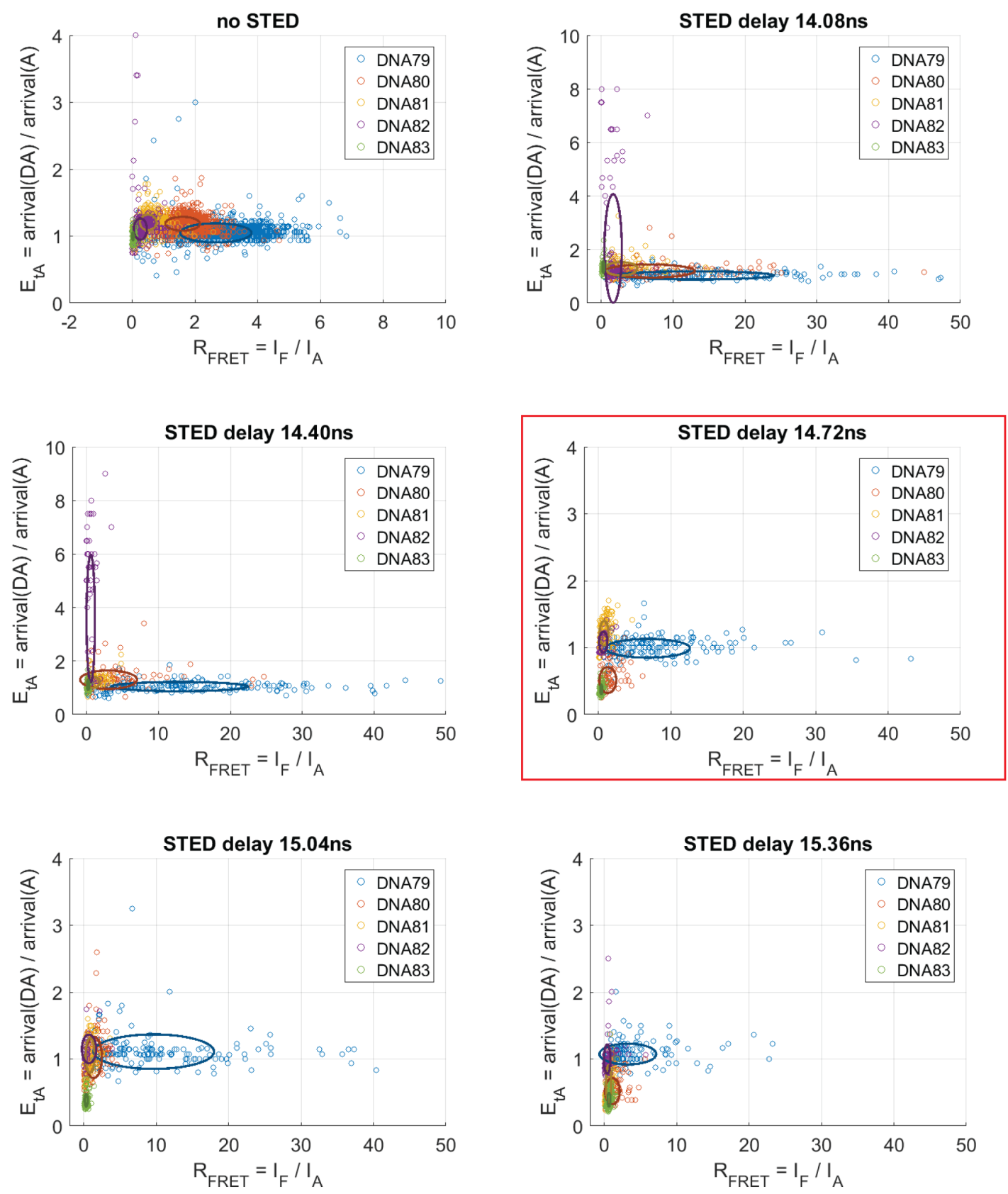

Fig. 28.5: Scatter plots with fluorescence intensity ratio $R_{\text {FRET }}$ and mean photon arrival time $E_{\mathrm{tA}}$ as coordinates for each single FRET pair. Over 150 individual FRET pairs of each FRET construct were measured with several different STED pulse delays. The delay value stated in ns refers to the delay of the STED trigger with respect to the excitation trigger and is converted to a.u. in table 14.1. The ellipses have a diameter of two s.d. of each distribution. 


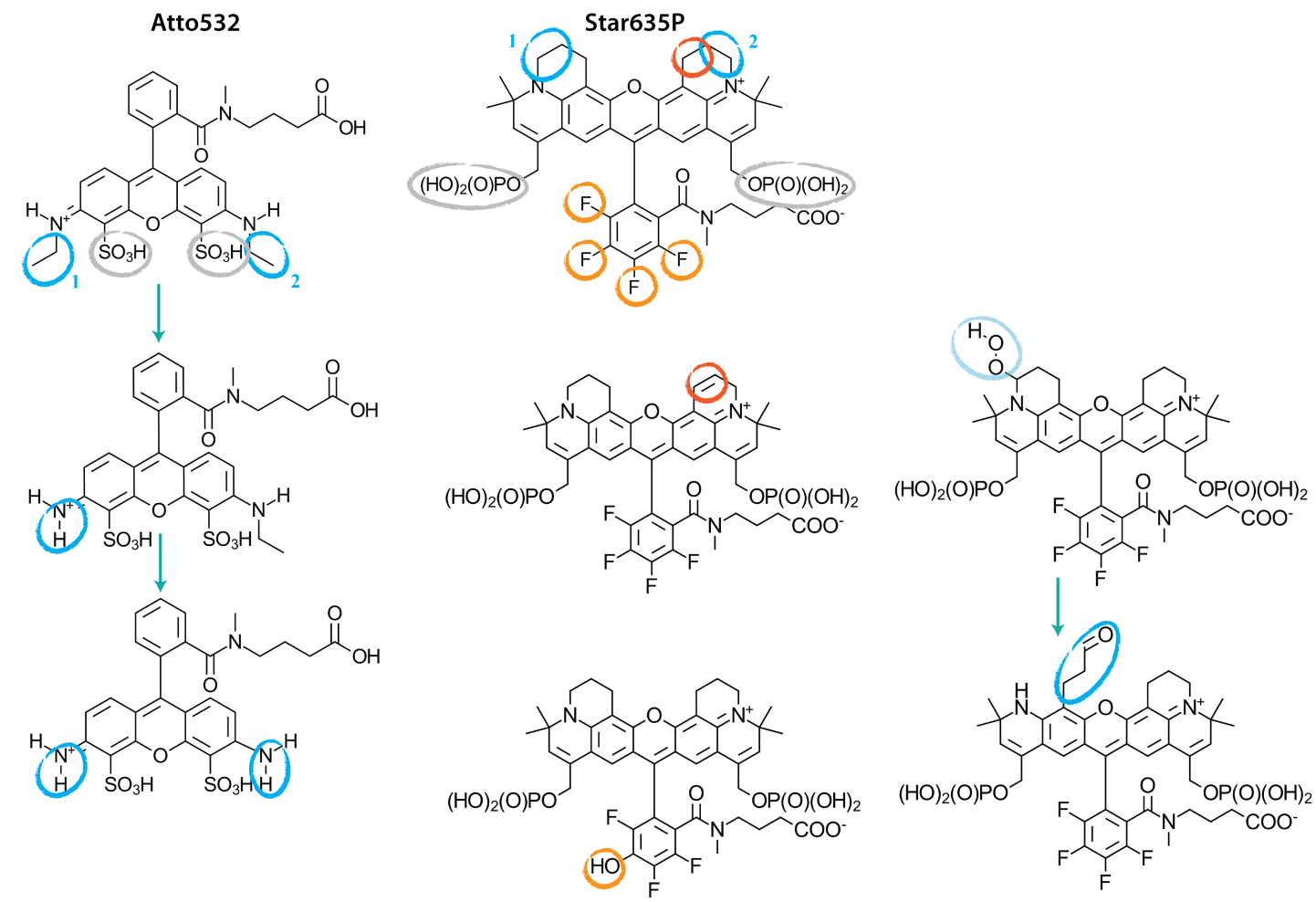

Fig. 28.6: Chemical structures of Atto532, Star635P and suggested photoproducts. The original structure of Atto532 was taken from [6], and that of Star635P from [144]. Possible photoproducts were suggested by Alexey Butkevich (personal communication, 12th December 2017). Structural changes resulting in an emission shift to shorter wavelengths are marked in blue. Structural changes that may cause an emission shift to longer wavelengths are marked in red (large shift) and orange (small shift). Groups that are less likely to be attacked and would result in an unknown spectral change are encircled in gray.

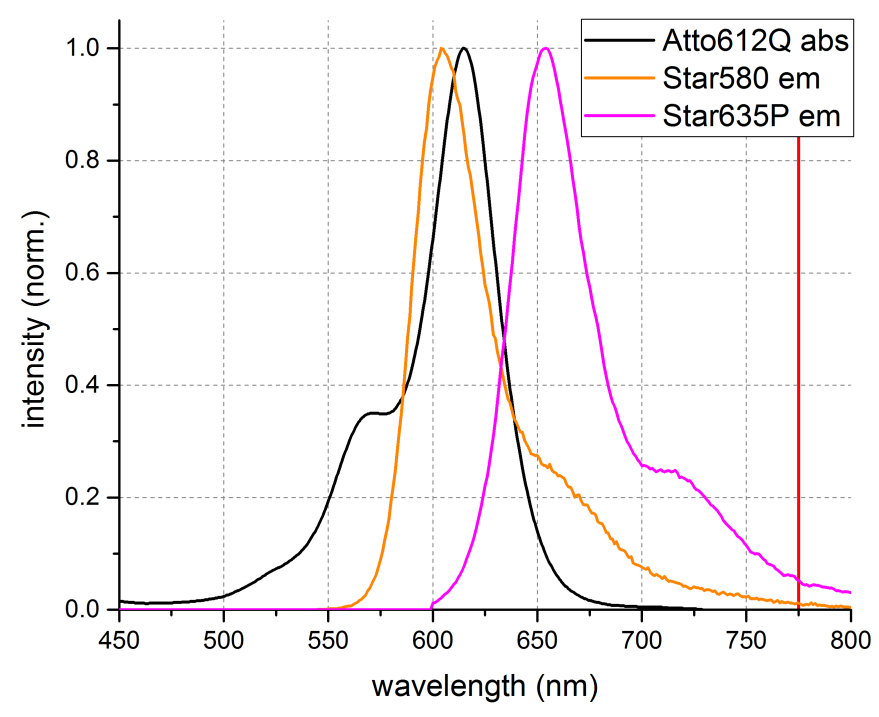

Fig. 28.7: The absorption spectrum of nonfluorescent quencher Atto612Q and emission spectra of Star580 and Star635P are shown in relation to the STED wavelength of $775 \mathrm{~nm}$. 

MATLAB codes 

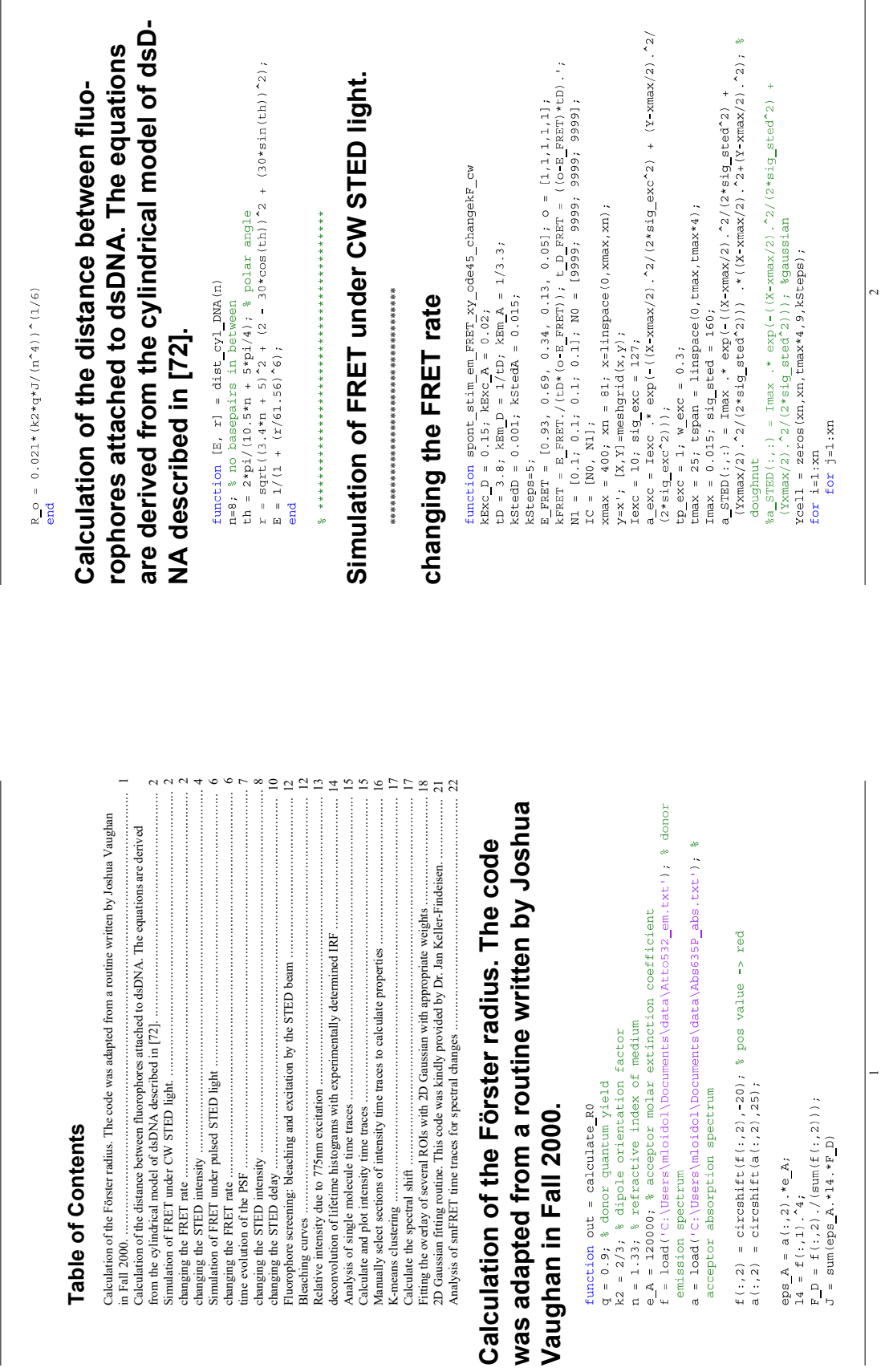

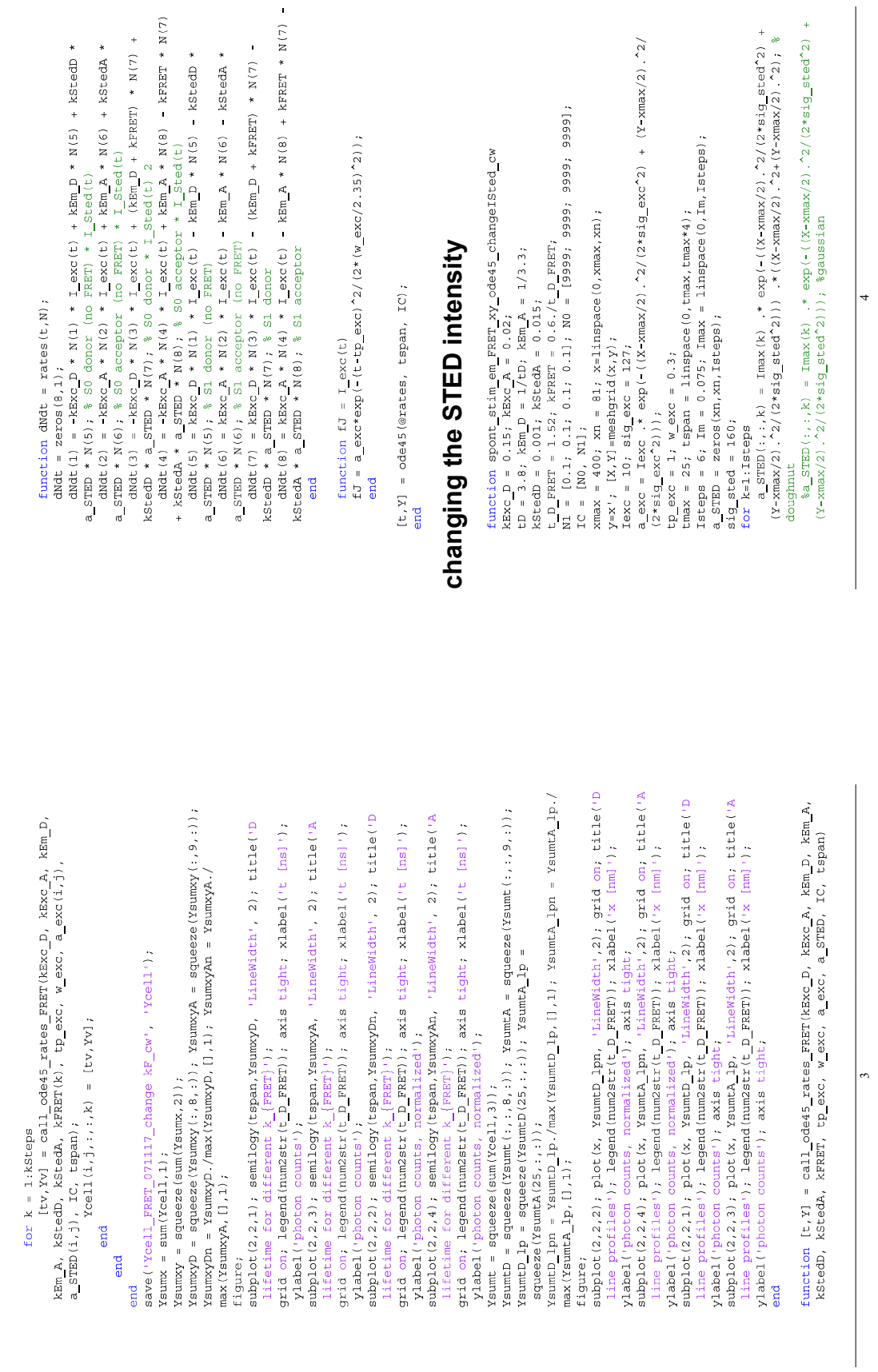

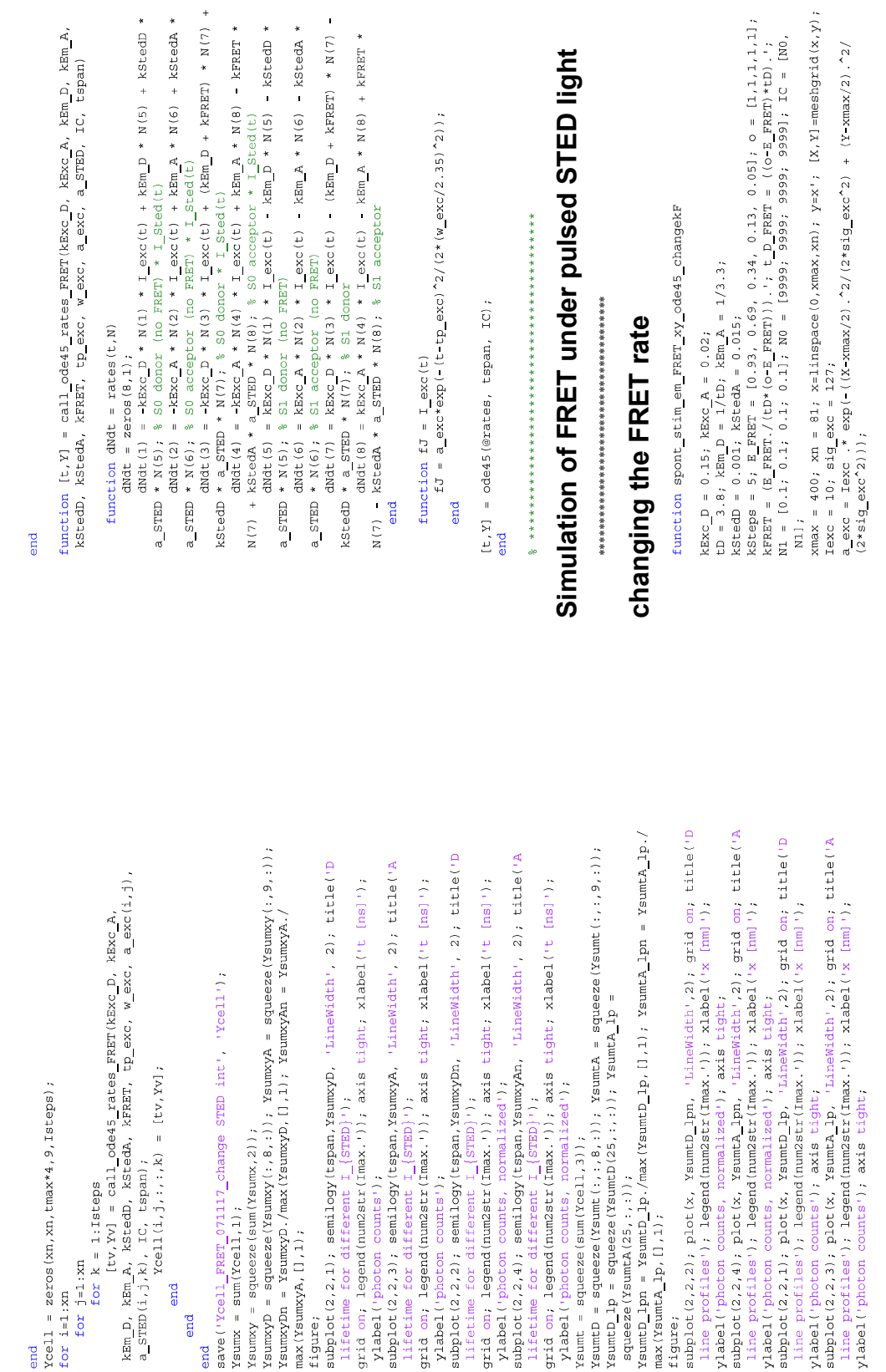

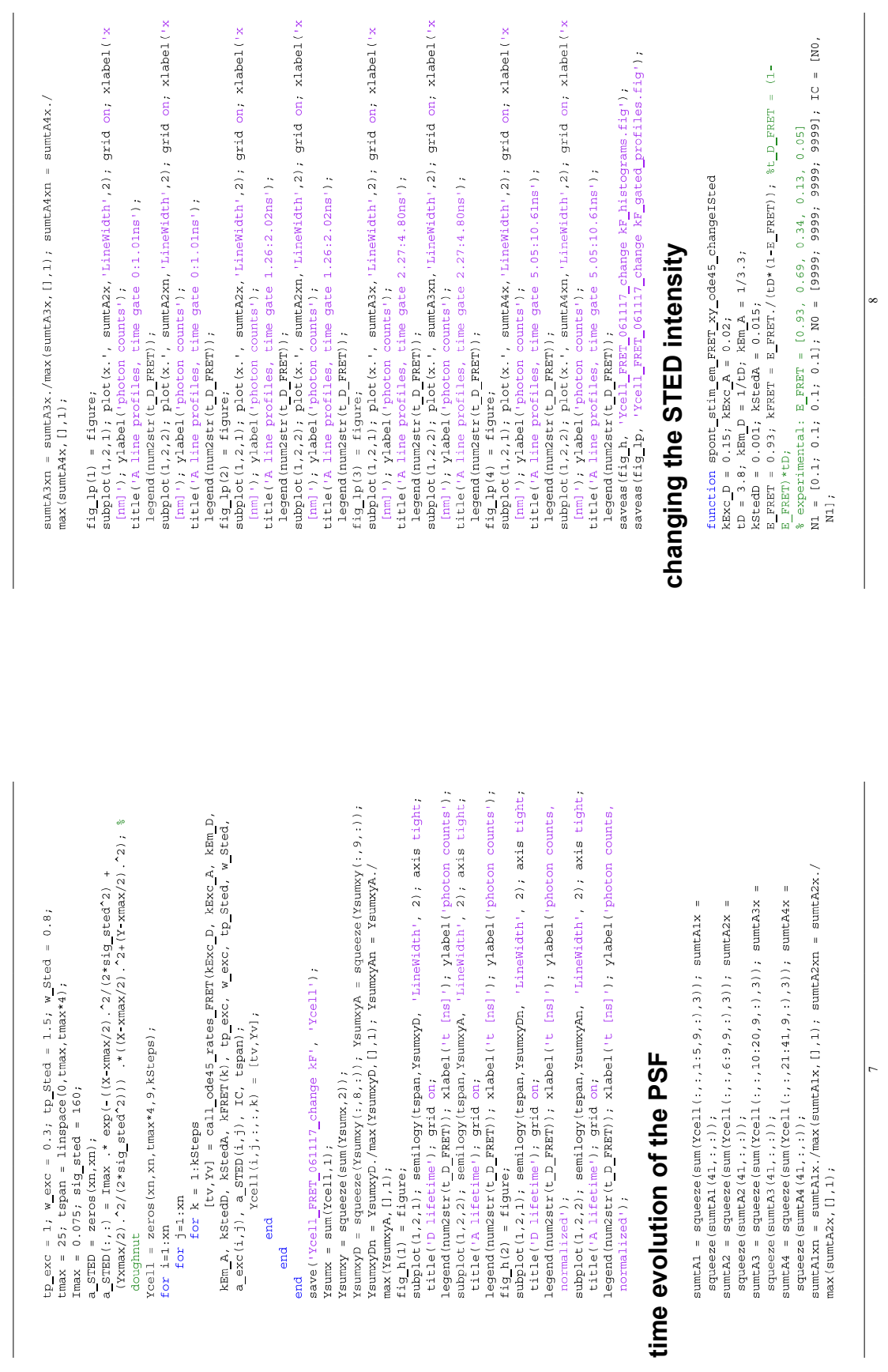

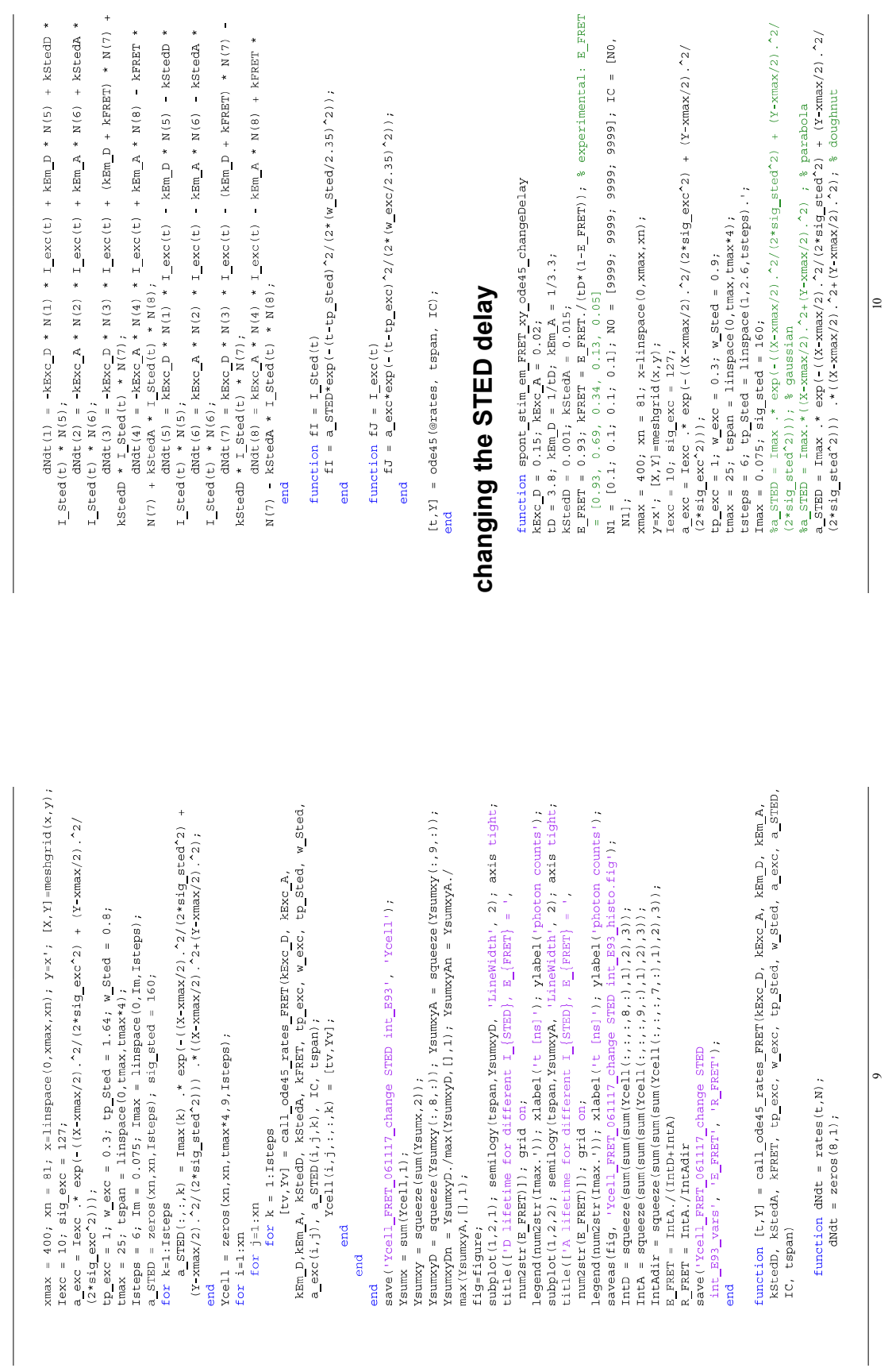

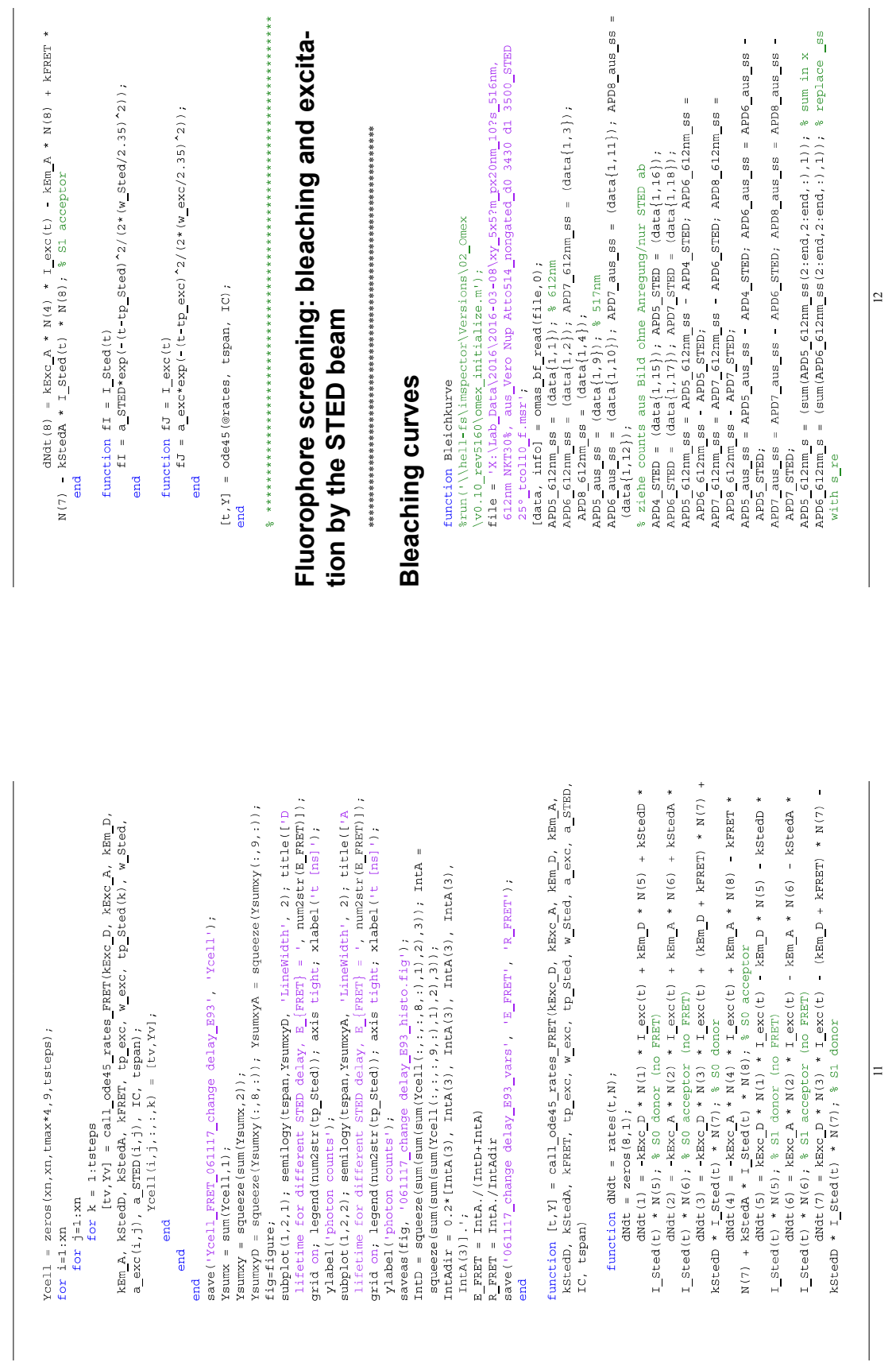

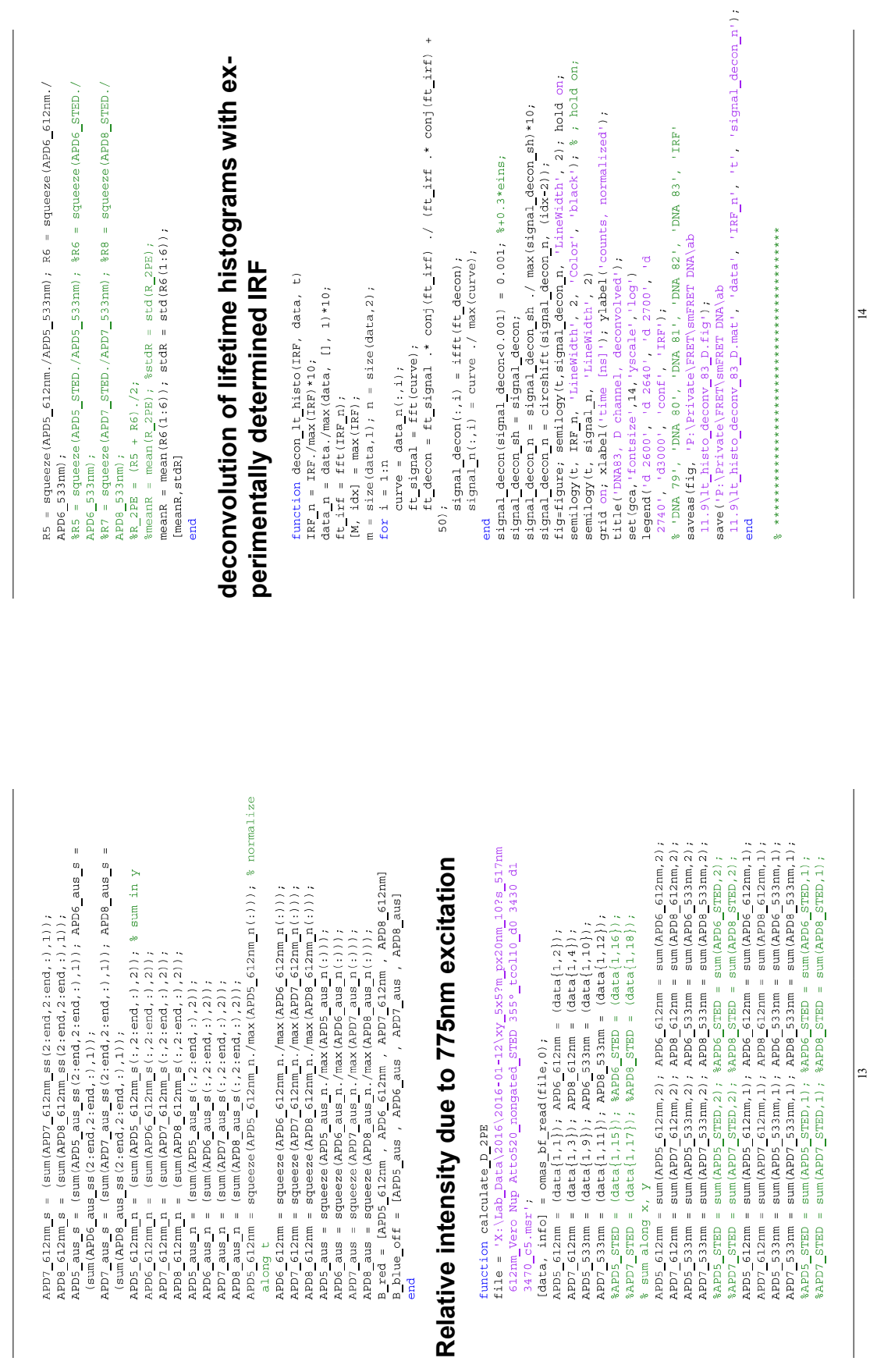

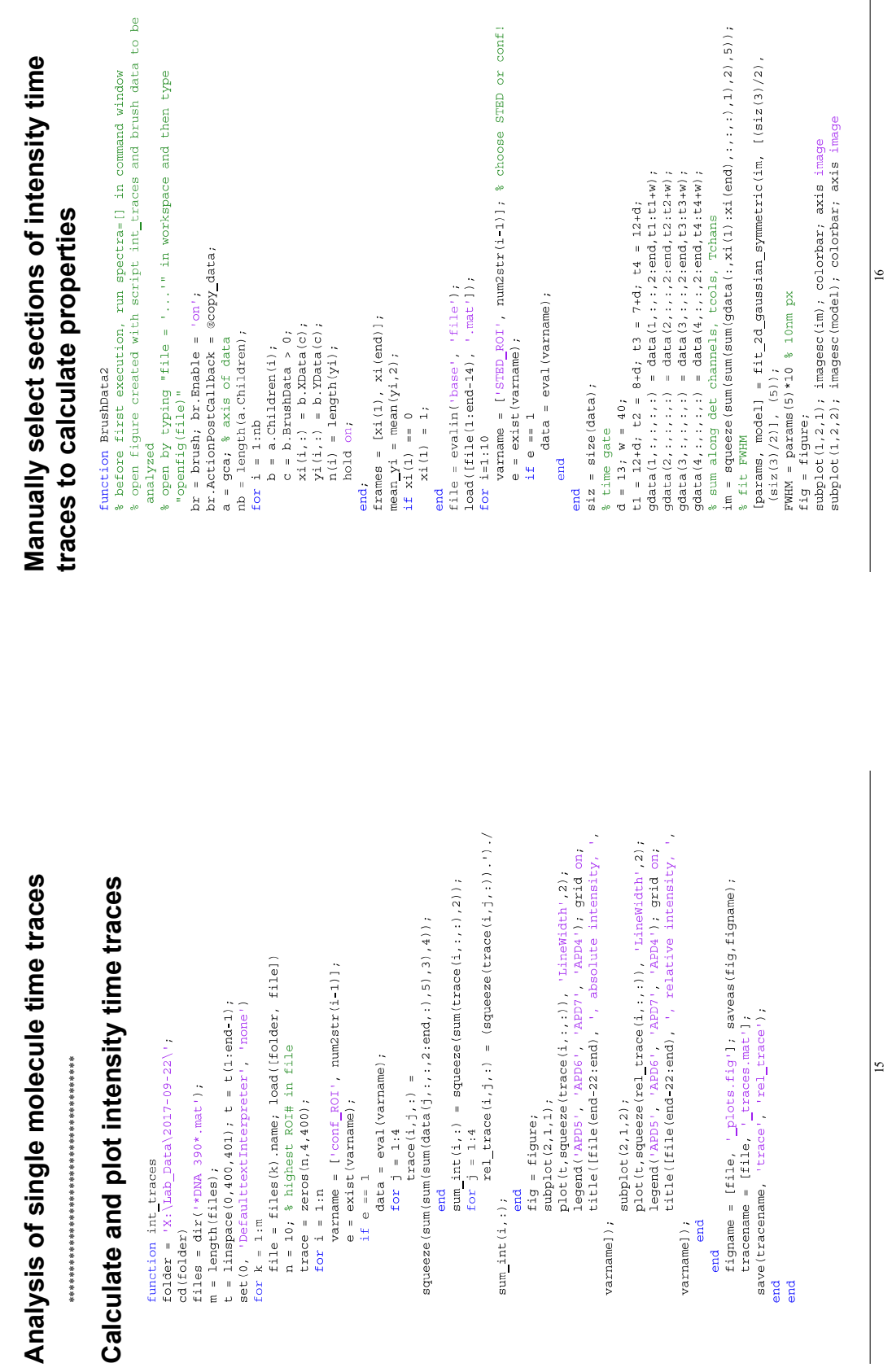

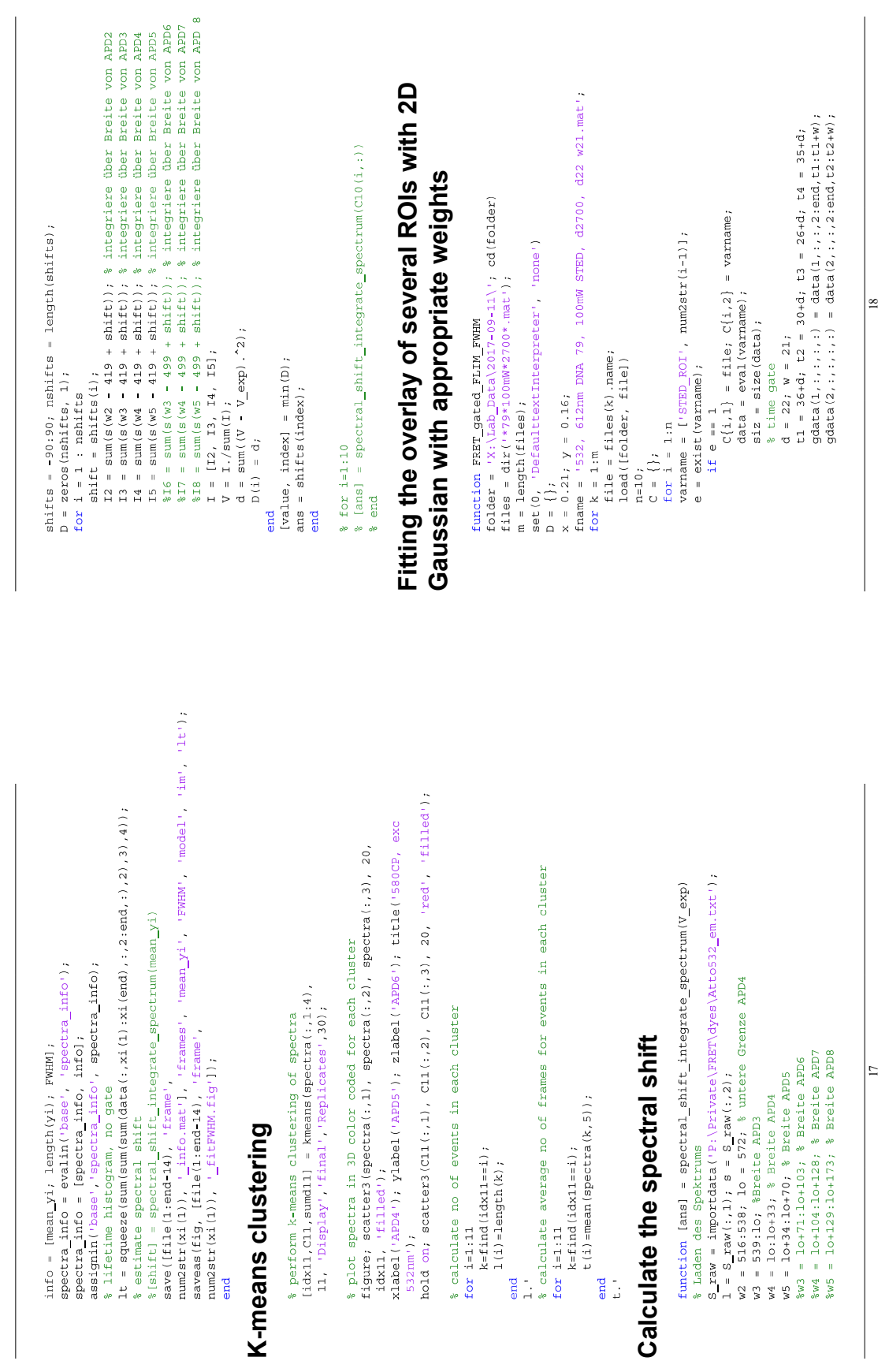

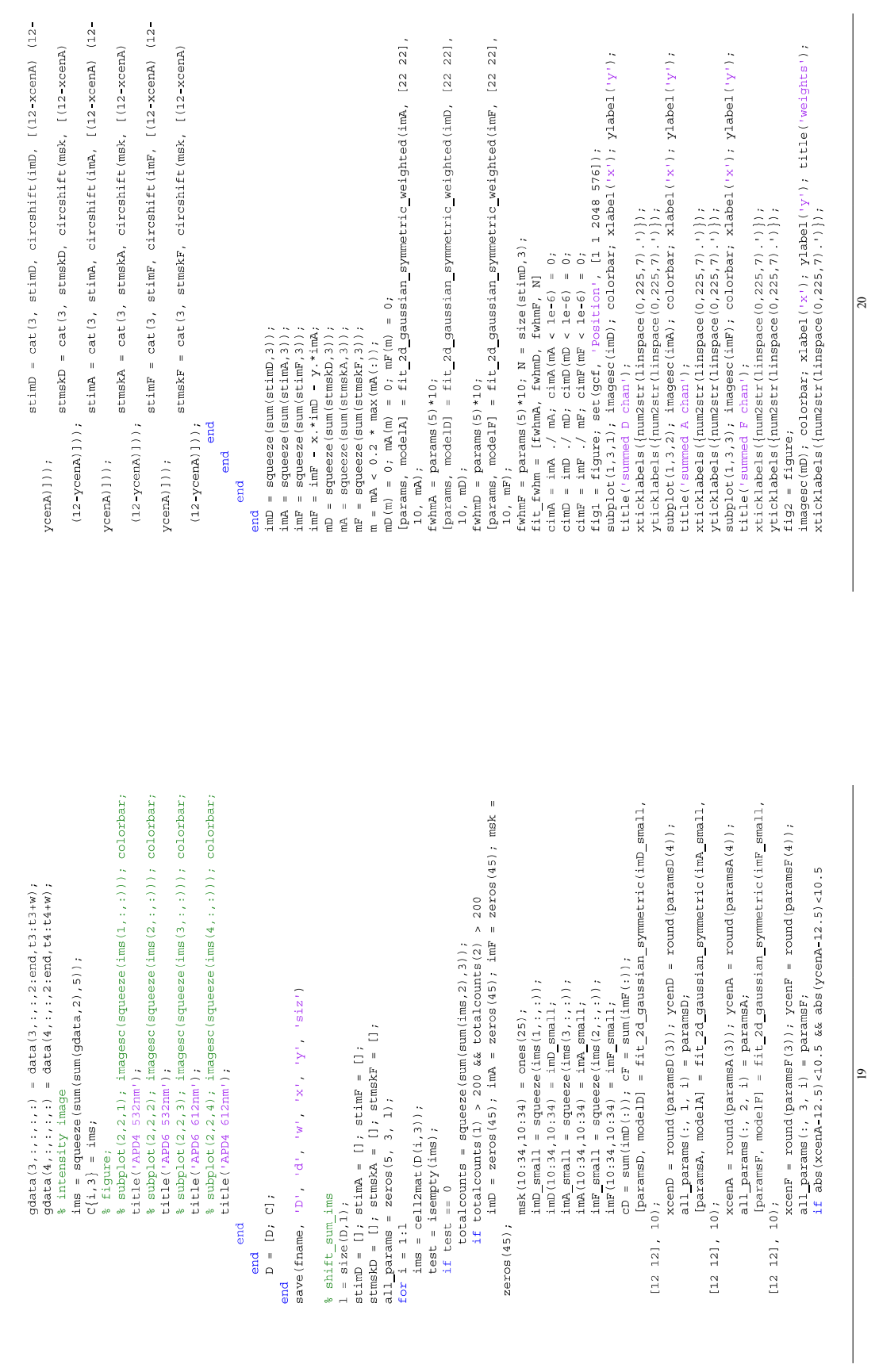

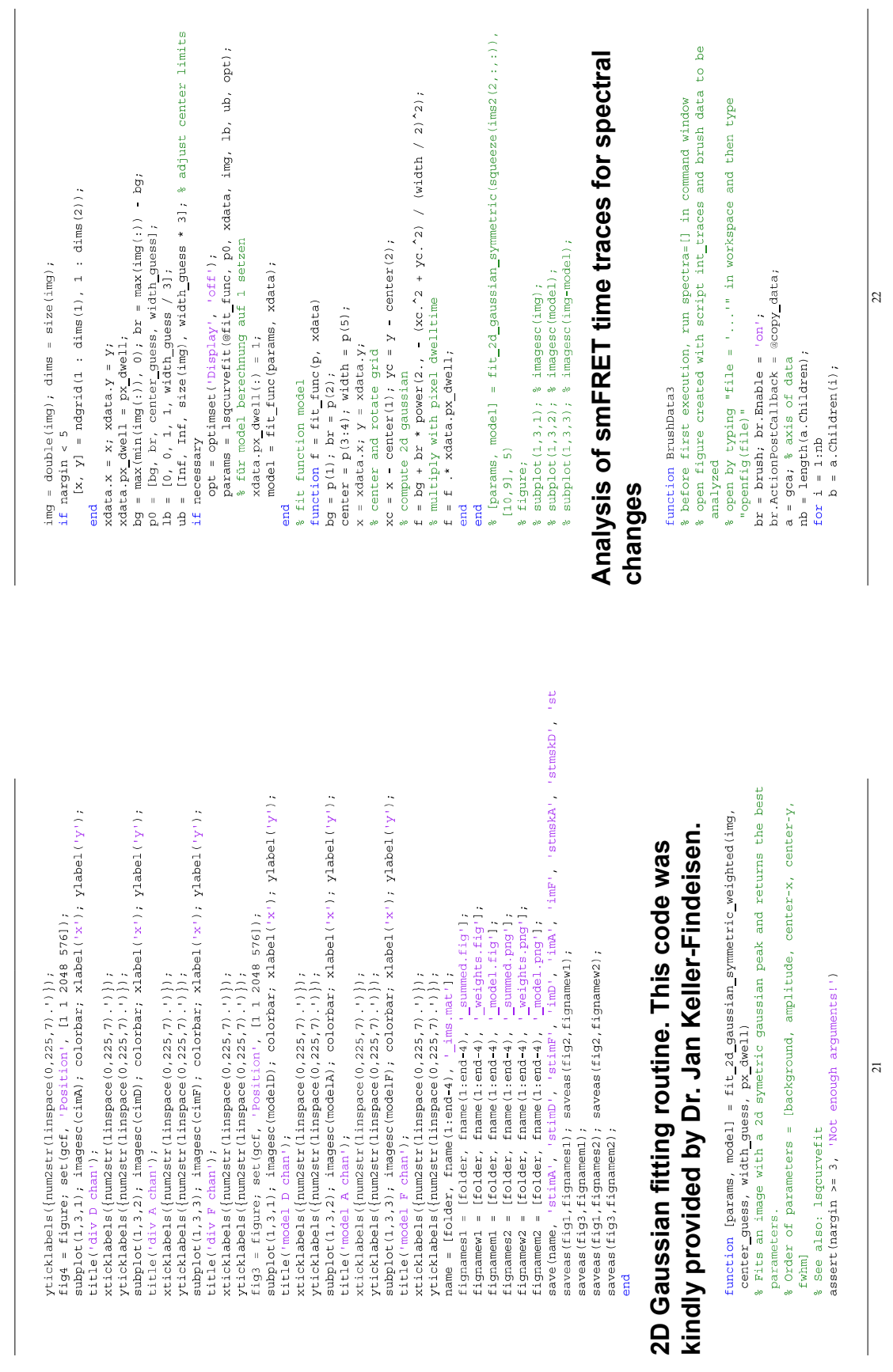


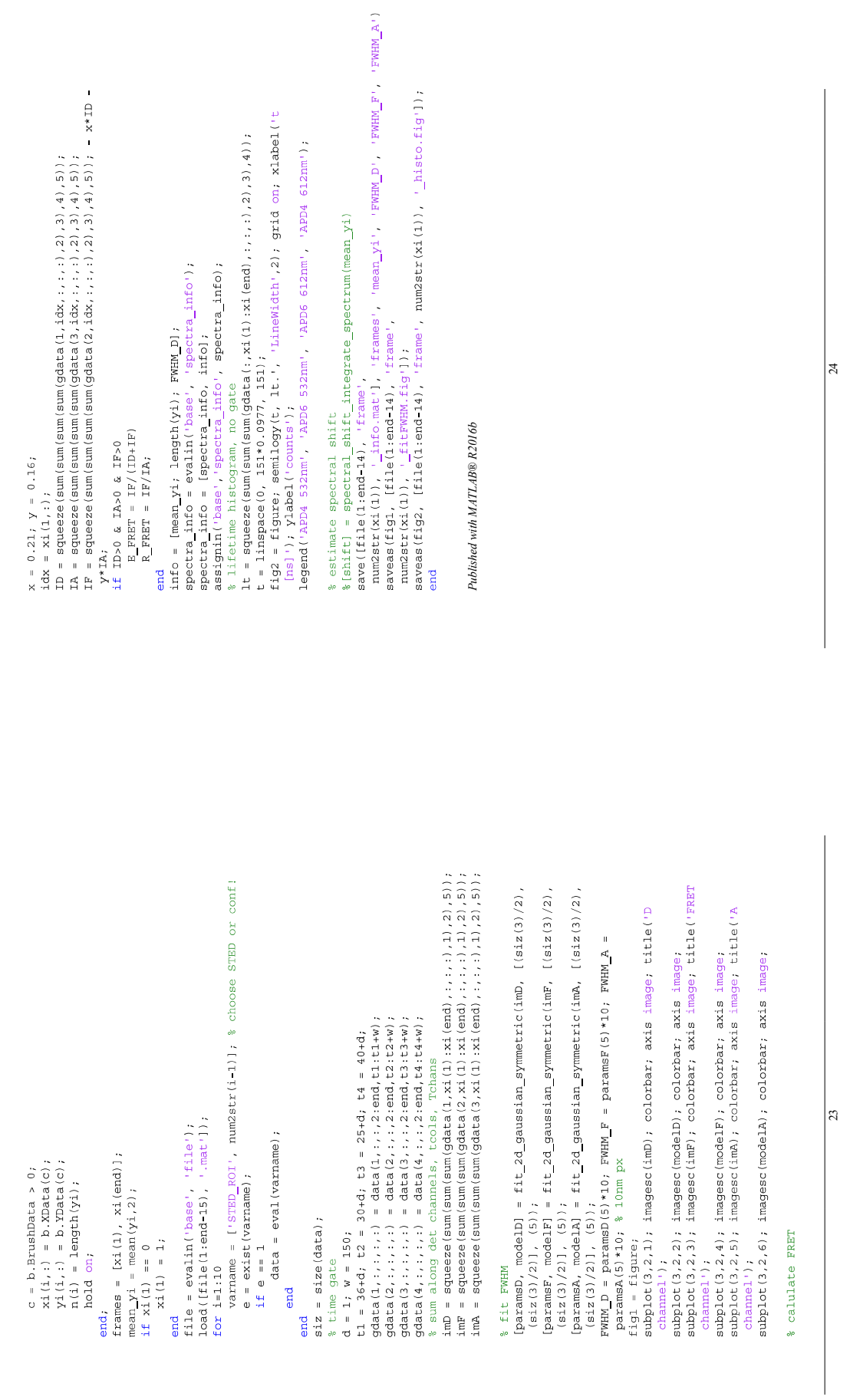



Python codes 
\#-*- coding: utf-8 -*-

Created on Fri Mar 17 16:08:05 2017

eauthor: mloidol

from future__import print_function

import specpy as $\mathrm{sp}$

from autoMinfield.source. autominfield. peak finder_new import peak finder process_stack from autominfield. source.autominfield.focus z keept import focus_z from scipy import io as sio

from scipy import
import datetim
import time

import time
import pickle

import matplotilib.pyplot as p1t
import pylab

sleep $=$ time. sleep

if _name_ = = "_main_":

\# connect to local Imspector

im $=$ s. s. Inspector ()
print ('Connected to Imspector () on (1'. format (im. version (), m. post $(1,1)$

\# Stack names of the confocal overview and the small scan image

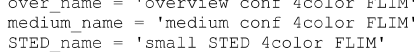

\# run a confocal overview scan at a predefined position

im.a activate (msr $\left.-{ }^{\circ}\right)$
time.sleep (0.1)

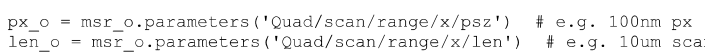

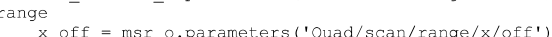

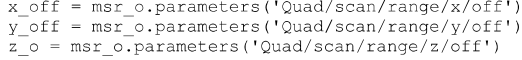

time.sleep $(0.1)$
im.run (mst 0$)$

\# get the data of the overview stack

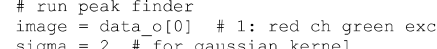

sigma $=2$
size $=12$ F for maxsinum filter
the $=0.7$ \# threshold for valid maxim

peaks, data_sm = peak_finder (image, sigma, size, thr

n_peaks = len (peaks $[0]$ )
print ('found od peaks in overview scan' $\frac{\mathrm{n}}{\mathrm{n}}$ peaks)

\#fig1 $1=$ plt. figure $(1)$
pyylab, imshow (image)

pylab. imshow(ima
ppylab. show ()

\#dict $=\{\}$
if $\mathrm{n}$,peaks $>10:$
n peaks $=10$

for is in range (n_peaks):

\# define ROT offset for intermediate scan

nss_ $m=$ im. measurement (medium name)

im. act ivate (msr $\left.{ }^{-2}\right)$
time. sleep $(0.1)$

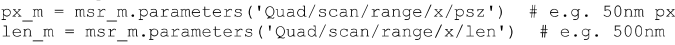

$\mathrm{X}_{-}=\mathrm{px}_{0} \mathrm{O} *$ peaks $[1][i]-1 \mathrm{en}-0 / 2+\mathrm{x}_{0}$ off $-50 \mathrm{e}-0 \mathrm{~g}$ \# positiv

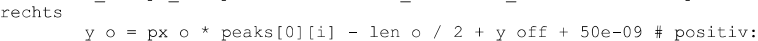

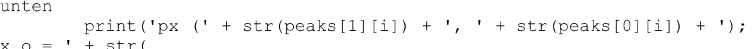

round $(x \circ * 1 \mathrm{e} 06,2))+1, y \circ={ }^{\prime}+\operatorname{str}(r o u n d(y \circ * 1 e 06$

\# run intermediate sca
mst_m. set_parameters $(1$ ?:

msr_m. set_parameters (' Quad/scan/range/z/off $1, z_{-}$o)

im. run (ms $-\mathrm{m})$
time. $\mathrm{sleep}(0.1)$

\# get the data of the intermediate stack
data_m, stack_titles_m $=$ process_stack (msr_m

summed $=$ False
while not summed:

$$
\begin{aligned}
& \text { Ery: } \\
& \text { ph_count }=\text { np.sum(data_m }[0], \text { axis=None) } \\
& \text { summed = True } \\
& \text { except RuntimeError: } \\
& \text { sleep (0.1) }
\end{aligned}
$$

Sleep (0.1)
print ('sleep_sum' 


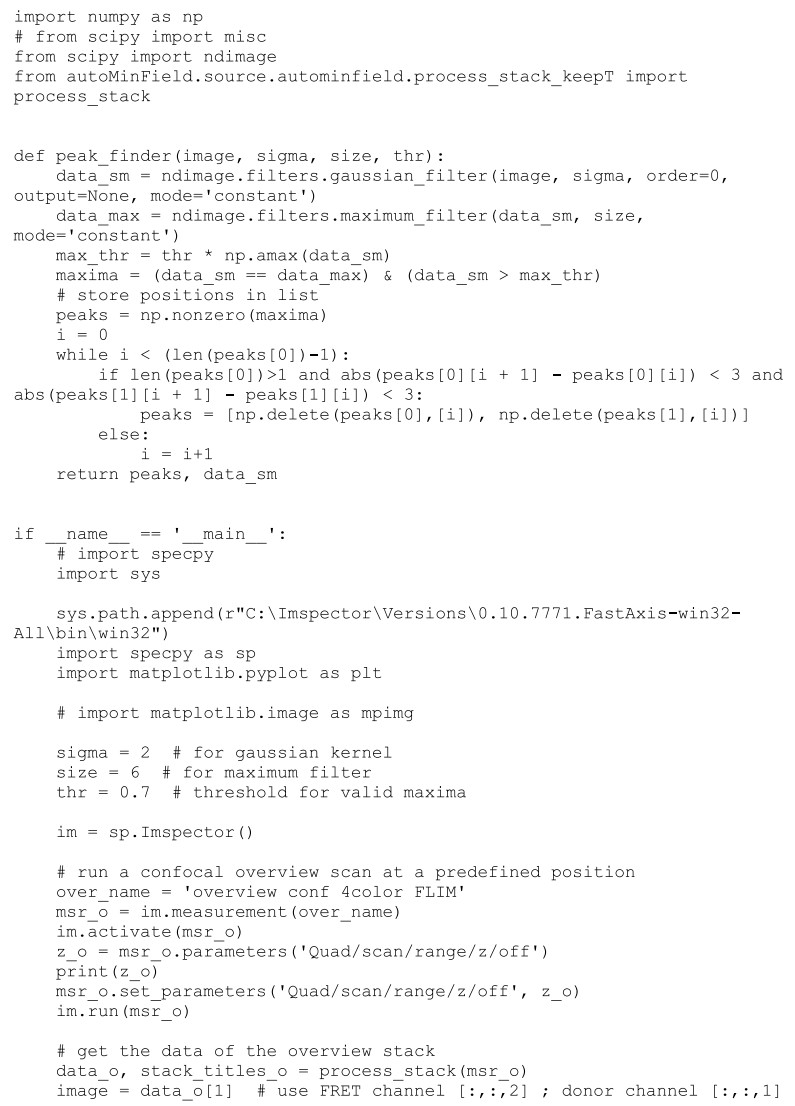
algorithms above to perform small scans at each position. 


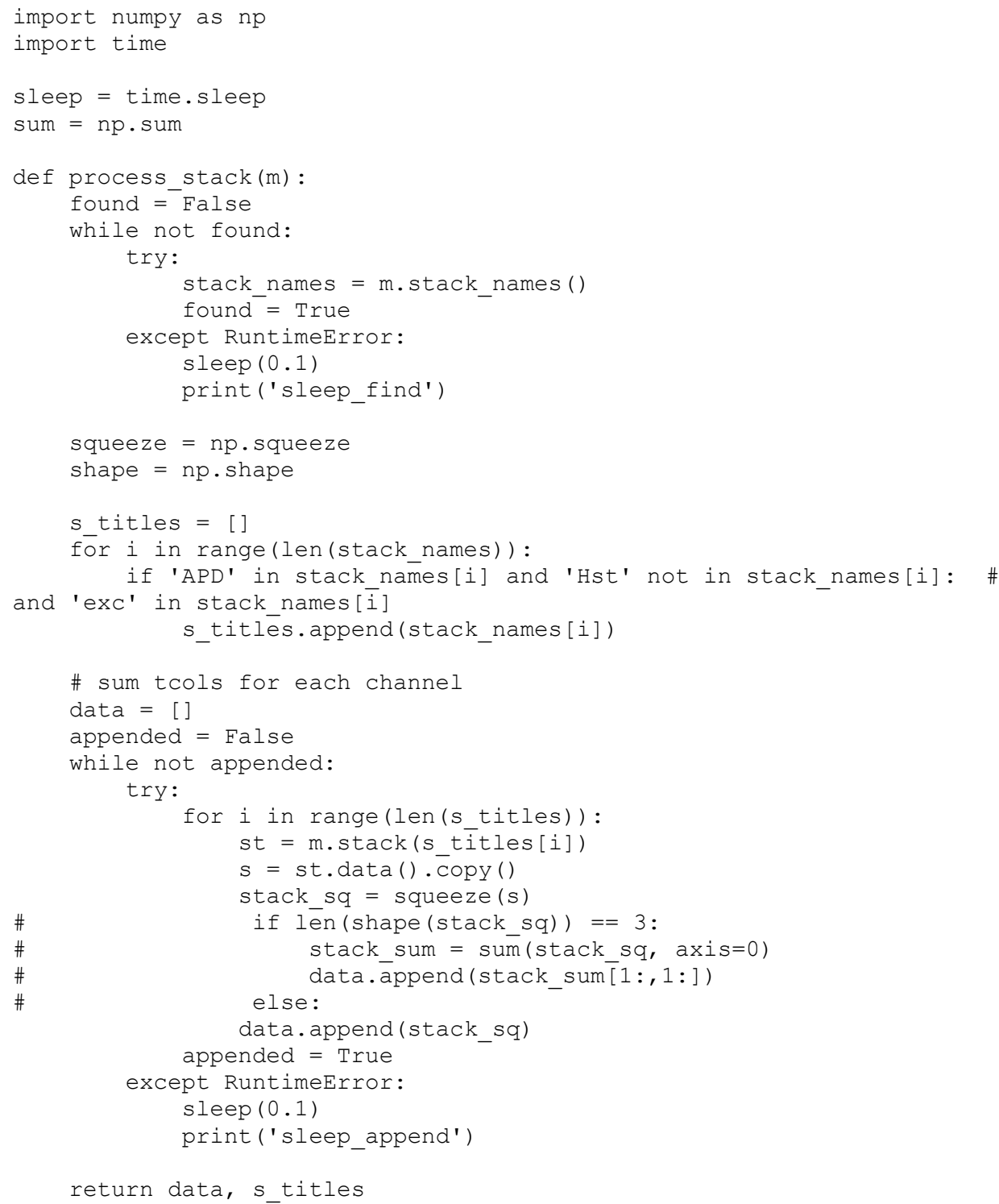

Fig. 30.4: This function extracts the image stacks from Imspector measurement files for further processing and saving in Python. 


\section{AOTF control scripts}

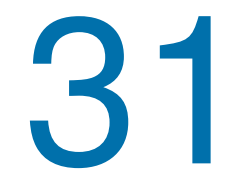

These scripts were put into the custom-written LabView software to select the wavelength and intensity of the excitation light. The frequency is given in $\mathrm{MHz}$ and can be converted to the wavelength in $\mathrm{nm}$ after calibration. The amplitude value and the intensity of the first diffraction order are not linearly related, but have to be calibrated once. For more information on the software, calibration, etc. refer to [80]. 


\section{$501.3 \mathrm{~nm} / 612.2 \mathrm{~nm}$}

AOTF1

dds frequency -p 0094

dds frequency -p 10104

dds frequency - 20115

dds frequency -p 30125

dds frequency -p 0180

dds frequency - 1199
dds frequency -p 21120

dds frequency -p 3196.18

dds frequency - 0290

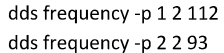

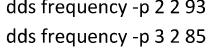

dds fsk *3

dds amplitude 08500

dds amplitude 12500

dds amplitude 20

dds amplitude 30

dds amplitude 40

dds amplitude 50

dds amplitude 60
AOTF3

dds frequency -p 008

dds frequency $-p 1091$

dds frequency $-\mathrm{p} 20135$

dds frequency - $\mathrm{p} 30125$

dds frequency - $p 01131$

dds frequency -p 11128

dds frequency - $p 2192$

dds frequency -p 3196.1

dds frequency -p 02112

dds frequency-p 1282

dds frequency - $\mathrm{p} 22102$

dds fsk *3 3

das tsk 3

dds amplitude 08500

dds amplitu 1850

dds amplitude 30

dds amplitude 30

dds amplitude 40

dds ampitiude 50

dds amplitude 60

\section{$510 \mathrm{~nm} / 612.4 \mathrm{~nm}$}

AOTF1

AOTF3

dds frequency -p 0094

dds frequency - $p 10104$

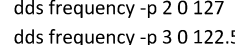

dds frequency $-\mathrm{p} 30122.5$
dds frequency $-\mathrm{p} 0180$

dds frequency - $p 0180$

dds frequency -p 1199
dds frequency -p 21116

dds frequency - 03196.18

dds frequency-p 3196.18
dds frequency-p 0290

dds frequency -p 0290

dds frequency-p 2293

dds frequency - 1285

dds fsk * 3

dds amplitude 08500

dds amplitude 13500

dds amplitude 20

dds amplitude 40

dds ampilitude 40

dds amplitude 50

dds ampilitude 60 dds frequency -p 0083

dds frequency -p 1091

dds frequency -p 20135

dds frequency $-p 30122.5$

dds frequency - 001131

dds frequency -p 11119

dds frequency $-p 2192$

dds frequency - $p 3196.18$

dds frequency -p 02113

dds frequency $-p 1282$

dds frequency -p 22102

dds frequency - 032107

dds fsk" 3

dds amplitude 08500

dds ampilude 18500

dds amplitude 20

dds amplitude 30

dds amplitude 40

dds amplitude 50

dds amplitude 70 
AOTF3

dds frequency -p 0094 dds frequency -p 10104 dds frequency $-\mathrm{p} 20124$ dds frequency - $\mathrm{p} 30120$ dds frequency -p 0180 dds frequency -p 119 dds frequency - 21117 dds frequency $-p 3196.18$ dds frequency - p 0290 dds frequency -p 12111 dds frequency -p 2293

dds frequency - $\mathrm{p} 328$ dds fsk *3

dds amplitude 08500

dds amplitude 13500

dds amplitude 20

dds amplitude 30

dds amplitude 40

dds amplitude 50

dds amplitude 60

dds amplitude 70 dds frequency - 00083

dds frequency $-p 1091$

dds frequency - $\mathrm{p} 20135$

dds frequency - $\mathrm{p} 30120$

dds frequency -p 01131

dds frequency -p 11127

dds frequency - 2192

dds frequency $-p 3196.18$

dds frequency -p 02114

dds frequency -p 1282

dds frequency - $\mathrm{p} 22102$

dds frequency -p 32107

dds fsk * 3

dds amplitude 08500

dds amplitude 18500

dds amplitude 20

dds amplitude 30

dds amplitude 40

dds amplitude 50

dds amplitude 60
dds amplitude 70

\section{$533 \mathrm{~nm} / 612.4 \mathrm{~nm}$}

AOTF1

AOTF3

dds frequency -p 0094

dds frequency -p 10104

dds frequency -p 20125

dds frequency - $\mathrm{p} 30115$

dds frequency -p 0180

dds frequency $-\mathrm{p} 11100$

dds frequency - $p 21120$

dds frequency - $p 3196.1$

dds frequency -p 0289

dds frequency -p 12130

dds frequency -p 2293

dds frequency -p 3285

dds fsk * 3

dds amplitude 08500

dds amplitude 17500

dds amplitude 20

dds amplitude 30

dds amplitude 40

dds amplitude 50

dds amplitude 60

dds amplitude 70 dds frequency -p 0083 dds frequency -p 1090 dds frequency $-\mathrm{p} 20135$ dds frequency - $\mathrm{p} 30115$ dds frequency - 01132 dds frequency - $\mathrm{p} 11127$ dds frequency - $p 2192$ dds frequency -p 3196.18 dds frequency - 02112 dds frequency - $p 1281$ dds frequency - 22102 dds frequency -p 32107 dds fsk *3

dds amplitude 08500

dds amplitude 18500

dds amplitude 20

dds amplitude 30

dds amplitude 40

dds amplitude 50

dds amplitude 60

dds amplitude 70 


\section{$612.4 \mathrm{~nm} /$ aus}

AOTF1

dds frequency - 0095

dds frequency -p 10104

dds frequency -p 20125

dds frequency -p 30107.7

dds frequency -p 0180

dds frequency -p 1199

dds frequency $-p 21120$

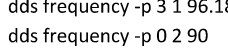

dds frequency -p 12113

dds frequency - $\mathrm{p} 2293$

dds frequency -p 328

dds fsk 3

dds amplitude 00

dds amplitude 15500

dds amplitude 20

dds amplitude 30

dds amplitude 40

dds ampltude 50

dds amplitude 60
AOTF3

dds frequency - 00083

dds frequency $-\mathrm{p} 109$

dds frequency - $\mathrm{p} 20135$

dds frequency $-\mathrm{p} 30100$

dds frequency $-p 01131$

dds frequency $-p 11127$

dds frequency - $p 2192$

dds frequency -p 3196.1

dds frequency - 022123

dds frequency $-\mathrm{p} 1282$

dds frequency - p 22102

dds frequency-p 32111

dds fsk 3

dds amplitude 00

dds amplitude 17000

dds amplitude 20

dds amplitude 30

dds amplitude 50

dds ampiltude 50

dds amplitude 60 


\section{Image acquisition parameters}

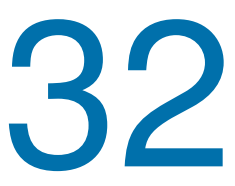

32.1 Green and red dye screening parameters 


\begin{tabular}{|c|c|c|c|c|c|c|c|c|c|c|c|c|c|c|c|}
\hline dye & date & sample & $\lambda_{\mathrm{exc}}(\mathrm{nm})$ & APDs & bleaching & $775 \mathrm{~nm}$ & STED focus & $d_{0}$ (a.u.) & $d_{1}$ (a.u.) & $p x_{x, y}$ & $\begin{array}{c}\mathrm{px} \\
(\mathrm{nm})\end{array}$ & $\begin{array}{l}\text { dwell } \\
(\mu \mathrm{s})\end{array}$ & lines & $\begin{array}{c}\text { frame } \\
(\mathrm{ms})\end{array}$ & $\begin{array}{l}\text { no. } \\
\text { frames }\end{array}$ \\
\hline Atto514 & 08.03 .2016 & Nup & 517,612, none & $3,4,6,7$ & $3,4,2: 10$ & $3,4,1: 10$ & gaussian & 3430 & 3500 & 250 & 20 & 10 & 3 & 1875 & 10 \\
\hline Atto520 & 12.01 .2016 & Nup & 517,612 & $3,4,5,6$ & $3,4,1: 10$ & $4,1: 6$ & gaussian & 3430 & 3470 & 250 & 20 & 10 & 2 & 1250 & 10 \\
\hline Atto532 & 01.02 .2016 & Nup & 517,612 , none & $3,4,6,7$ & $3,4,2: 10$ & $3,4,1: 10$ & gaussian & 3430 & 3470 & 250 & 20 & 10 & 3 & 1875 & 10 \\
\hline Atto532 & 12.01 .2016 & Nup & 517,612 & $3-6$ & $3,4,1: 10$ & $4,1: 10$ & gaussian & 3430 & 3470 & 250 & 20 & 10 & 2 & 1250 & 10 \\
\hline Atto532 & 19.01 .2016 & Nup & 17,612, none & $3,4,6,7$ & $3,4,6,1: 10$ & $3,4,6,1: 6$ & gaussian & 3430 & 3500 & 251 & 20 & 10 & 3 & 1890 & 10 \\
\hline Atto532 & 13.08 .2015 & Nup & 533,612 & $4,6,7,8$ & $4,1: 10$ & $4,6,7,1: 5$ & doughnut & 3320 & - & 250 & 20 & 10 & 2 & 1250 & 5 \\
\hline Atto542 & 29.02 .2016 & Nup & 533,612 , none & $4-7$ & $4-7,1: 10$ & $4-6,1: 5$ & gaussian & 3430 & 3470 & 250 & 20 & 10 & 3 & 1875 & 10 \\
\hline Atto550 & 12.01 .2016 & Nup & 517,612 & $3-6$ & $3,4,2: 10$ & $4-6,1: 10$ & gaussian & 3430 & 3470 & 250 & 20 & 10 & 2 & 1250 & 10 \\
\hline Atto550 & 19.01 .2016 & Tub & 517,612 , none & $3,4,6,7$ & $3,4,6,1: 10$ & $3,4,6,1: 10$ & gaussian & 3430 & 3500 & 251 & 20 & 10 & 3 & 1890 & 10 \\
\hline Atto550 & 13.08 .2015 & Nup & 533,612 & $4,6,7,8$ & $4,1: 10$ & $4,6,7,1: 5$ & doughnut & 3320 & . & 250 & 20 & 10 & 2 & 1250 & 5 \\
\hline Atto565 & 12.01 .2016 & Nup & 517,612 & $3-6$ & $3-6,2: 10$ & $4-6,1: 10$ & gaussian & 3430 & 3470 & 250 & 20 & 10 & 2 & 1250 & 10 \\
\hline Atto565 & 19.01 .2016 & Nup & 517,612 , none & $3,4,6,7$ & $3,4,6,1: 10$ & $3,4,6,1: 6$ & gaussian & 3430 & 3500 & 251 & 20 & 10 & 3 & 1890 & 10 \\
\hline Alexa514 & 29.02.2016 & Mito & 517, none & $3-6$ & $3-6,2: 10$ & $3-6,1: 10$ & gaussian & 3430 & 3470 & 250 & 20 & 10 & 2 & 1250 & 10 \\
\hline Alexa532 & 01.02 .2016 & Nup & 517,612 , none & $3,4,6,7$ & $3,4,2: 10$ & $3,4,1: 6$ & gaussian & 3430 & 3470 & 250 & 20 & 10 & 3 & 1875 & 10 \\
\hline Alexa546 & 29.02.2016 & Nup & 533,612, none & $4-7$ & $4-7,1: 10$ & $4,5,1: 3$ & gaussian & 3430 & 3470 & 250 & 20 & 10 & 3 & 1875 & 10 \\
\hline Alexa555 & 29.02 .2016 & Nup & 533,612, none & $4-7$ & $4-7,2: 10$ & $4-6,1: 3$ & gaussian & 3430 & 3470 & 250 & 20 & 10 & 3 & 1875 & 10 \\
\hline Star512 & 01.02 .2016 & Nup & 517,612 , none & $3,4,6,7$ & $3,4,2: 10$ & $3,4,1: 7$ & gaussian & 3430 & 3470 & 250 & 20 & 10 & 3 & 1875 & 10 \\
\hline DyL485LS & 03.03 .2016 & Nup & 502,612, none & $3,4,6,7$ & $3,4,2: 10$ & $3,4,1: 10$ & gaussian & 3430 & 3530 & 250 & 20 & 10 & 3 & 1875 & 10 \\
\hline Dy485XL & 03.03 .2016 & Nup & 502,612, none & $3,4,6,7$ & $3,4,2: 10$ & $3,4,1: 10$ & gaussian & 3430 & 3530 & 250 & 20 & 10 & 3 & 1875 & 10 \\
\hline DyL633 & 18.03 .2015 & Nup & 571,656 & $5-8$ & $6-8,1: 10$ & - & doughnut & 3175 & - & 500 & 20 & 10 & 2 & 5000 & 10 \\
\hline Star635P & 18.03 .2015 & Nup & 571,656 & $5-8$ & $6-8,1: 10$ & - & doughnut & 3175 & - & 500 & 20 & 10 & 2 & 5000 & 10 \\
\hline StarRed & 17.03 .2015 & Nup & 571,656 & $5-8$ & $6-8,1: 10$ & - & doughnut & 3175 & - & 500 & 20 & 10 & 2 & 5000 & 10 \\
\hline Atto633 & 17.03 .2015 & Nup & 571,656 & $5-8$ & $6-8,1: 10$ & - & doughnut & 3175 & - & 500 & 20 & 10 & 2 & 5000 & 10 \\
\hline
\end{tabular}

Tab. 32.1: Samples for screening green and red dyes. Different structures in cultured Vero cells, namely nuclear pore complex protein Nup153 (Nup), tubulin (Tub) or mitochondrial protein Tom20 (Mito) were stained with dye-labeled antibodies. Stated are: the days on which the measurements were performed, excitation wavelengths, detectors, STED beam parameters and scan parameters. A gaussian-shaped STED focus was created by removing the phase plate from the beam path. In the "bleaching" column, it is stated which detection channels and frames were evaluated for obtaining the bleaching rate. In the "775nm" column the detection channels and frames which were used to calculate the excitation of the dye by the 775nm STED beam are listed. The number of times each line of the image was scanned, namely once with each excitation wavelength, is given in column "lines". The number of pixels in $\mathrm{x}$ and $\mathrm{y}$, the dwell time per pixel and the number of line steps were multiplied to obtain the duration of each frame. The scanner flyback was omitted because its duration is not known. 


\subsection{Scan parameters for single molecule photoconversion and single pair FRET experiments}




\begin{tabular}{|c|c|c|c|c|c|c|c|c|c|c|c|c|c|}
\hline dye & date & $\lambda_{\mathrm{exc}}(\mathrm{nm})$ & APDs & STED & $d_{0}$ (a.u.) & $d_{1}$ (a.u.) & $p x_{x}$ & $p x_{y}$ & $\mathrm{px}(\mathrm{nm})$ & $\begin{array}{c}\text { dwell } \\
(\mu \mathbf{s})\end{array}$ & $\begin{array}{c}\text { frames } \\
\text { (ms) }\end{array}$ & $\begin{array}{c}\text { no. } \\
\text { frames }\end{array}$ & $\begin{array}{c}\text { histogram } \\
\text { bins }\end{array}$ \\
\hline Atto532 & $\begin{array}{l}\text { 26.06.2017, } \\
\text { 19.07.2017 }\end{array}$ & 510 & $2-5$ & no STED & - & - & 9 & 8 & 50 & 70 & 5.04 & 400 & 137 \\
\hline Star635P & $\begin{array}{l}\text { 26.06.2017 } \\
\text { 19.07.2017 }\end{array}$ & 552 & $5-8$ & no STED & - & - & 9 & 8 & 50 & 70 & 5.04 & 400 & 137 \\
\hline Star635P & 22.09 .2017 & 552 & $4-7$ & $\begin{array}{l}\text { doughnut, } \\
75 \mathrm{~mW}\end{array}$ & 3220 & 2640 & 26 & 25 & 10 & 30 & 19.5 & 150 & 100 \\
\hline $\begin{array}{c}\text { Atto532 } \\
\text { \& Star635P }\end{array}$ & $\begin{array}{c}08.08 . \\
-17.08 .2017\end{array}$ & 532,612 & 4,6 & no STED & - & - & 10 & 9 & 50 & 50 & 4.5 & 10 & 137 \\
\hline $\begin{array}{c}\text { Atto532 } \\
\text { \& Star635P }\end{array}$ & $\begin{array}{c}08.08 . \\
-17.08 .2017\end{array}$ & 532,612 & 4,6 & $\begin{array}{l}\text { doughnut, } \\
25-175 \mathrm{~mW}\end{array}$ & 3220 & $2600-3040$ & 8 & 7 & 15 & 30 & 1.68 & 100 & 137 \\
\hline $\begin{array}{c}\text { Atto532 } \\
\text { \& Star635P }\end{array}$ & $\begin{array}{c}11.09 . \\
-21.09 .2017\end{array}$ & 532,612 & 4,6 & no STED & - & - & 10 & 9 & 50 & 50 & 4.5 & 10 & 256 \\
\hline $\begin{array}{c}\text { Atto532 } \\
\text { \& Star635P }\end{array}$ & $\begin{array}{c}11.09 \\
-21.09 .2017\end{array}$ & 532,612 & 4,6 & $\begin{array}{l}\text { doughnut, } \\
25-100 \mathrm{~mW}\end{array}$ & 3220 & $2600-3000$ & 26 & 25 & 10 & 25 & 16.25 & 50 & 256 \\
\hline
\end{tabular}

Tab. 32.2: Experimental settings to test single dyes Atto532 and Star635P for photoconversion and to measure FRET between single pairs of Atto532 \& Star635P. Stated are: the days on which the measurements were performed, excitation wavelengths, detectors, STED parameters, scan settings, and the number of lifetime histogram bins. For the data set acquired in August 2017, spectral correction factors $x=0.19$ (D bleedthrough) and $\mathrm{y}=0.16$ (A direct excitation) were measured with singly labeled DNA constructs. For the data set acquired in September 2017, $\mathrm{x}=0.21$ and $\mathrm{y}=0.16$ were obtained. 


\section{Bibliography}

[1]Alison Abbott. „Microscopic marvels: Seeing the system“. In: Nature News 459.7247 (2009), pp. 630-631 (cit. on p. 22).

[2]W. P. Ambrose, Th. Basché, and W. E. Moerner. „Detection and spectroscopy of single pentacene molecules in a p-terphenyl crystal by means of fluorescence excitation". In: The Journal of Chemical Physics 95.10 (1991), pp. 7150-7163 (cit. on pp. 12, 13, 135).

[3]M. Arrio-Dupont, G. Foucault, M. Vacher, P. F. Devaux, and S. Cribier. „Translational diffusion of globular proteins in the cytoplasm of cultured muscle cells". In: Biophys $J$ 78.2 (2000), pp. 901-7 (cit. on p. 160).

[4]Alexander Auer, Maximilian Thomas Strauss, Thomas Schlichthaerle, and Ralf Jungmann. „Fast, background-free DNA-PAINT imaging using FRET-based probes“. In: Nano Letters () (cit. on pp. 39, 155).

[5]Haim Yuval Aviram, Menahem Pirchi, Yoav Barak, Inbal Riven, and Gilad Haran. „Two states or not two states: Single-molecule folding studies of protein L“. In: The Journal of Chemical Physics 148.12 (2018), p. 123303 (cit. on p. 34).

[6]Murat Aydemir, Vygintas Jankus, Fernando B. Dias, and Andy Monkman. „The key role of geminate electron-hole pair recombination in the delayed fluorescence in rhodamine 6G and ATTO-532“. In: Phys. Chem. Chem. Phys. 16 (39 2014), pp. 21543-21549 (cit. on pp. 137, 185).

[7]Arjen N. Bader, Erik G. Hofman, Jarno Voortman, Paul M. P. van Bergen en Henegouwen, and Hans C. Gerritsen. „Homo-FRET Imaging Enables Quantification of Protein Cluster Sizes with Subcellular Resolution“. In: Biophysical Journal 97 (2009), 2613-2622 (cit. on p. 154).

[8]Bryce T. Bajar, Emily S. Wang, Shu Zhang, Michael Z. Lin, and Jun Chu. „A Guide to Fluorescent Protein FRET Pairs“. In: Sensors 16.9 (2016), pp. 1488-1-1488-24 (cit. on p. 170).

[9]Vincenzo Balzani, Paola Ceroni, and Alberto Juris. Photochemistry and photophysics: concepts, research, applications. John Wiley \& Sons, 2014 (cit. on pp. 6, 7).

[10]Francisco Balzarotti, Yvan Eilers, Klaus C. Gwosch, et al. „Nanometer resolution imaging and tracking of fluorescent molecules with minimal photon fluxes". In: Science (2016). eprint: http : / / science. sciencemag . org/ content / early/2016/12/21/science . aak9913.full.pdf (cit. on p. 24). 
[11]Manu Ben-Johny, Daniel N. Yue, and David T. Yue. „Detecting stoichiometry of macromolecular complexes in live cells using FRET“. In: Nature Communications 7 (2016), pp. 13709-1 -13709-10 (cit. on pp. 34, 151, 152).

[12]Fabian Bergermann. „Massively Parallelized STED Nanoscopy“. Dissertation. 2016 (cit. on p. 168).

[13]Sebastian Berning, Katrin I. Willig, Heinz Steffens, Payam Dibaj, and Stefan W. Hell. „Nanoscopy in a Living Mouse Brain“. In: Science 335.6068 (2012), pp. 551-551. eprint: http://science.sciencemag.org/content/335/6068/551.full.pdf (cit. on p. 34).

[14]Shivaprasad Bhuvanendran, Kyle Salka, Kristin Rainey, et al. „Superresolution Imaging of Human Cytomegalovirus vMIA Localization in Sub-Mitochondrial Compartments“. In: Viruses 6.4 (2014), pp. 1612-1636 (cit. on p. 33).

[15]Gerald E. Blair, J. Bradford Merry, and James M. Wylot. Acousto-optic device and method using tellurite glass composition. US Patent 4,265,517. 1981 (cit. on p. 176).

[16]Marie N. Bongiovanni, Julien Godet, Mathew H. Horrocks, et al. „Multi-dimensional superresolution imaging enables surface hydrophobicity mapping“. In: Nature Communications 7 (2016), p. 13544 (cit. on p. 13).

[17]Tanja Brakemann, Andre C. Stiel, Gert Weber, et al. „A reversibly photoswitchable GFP-like protein with fluorescence excitation decoupled from switching". In: Nature Biotechnology 29.10 (2011), pp. 942-947 (cit. on pp. 158, 170).

[18]Stefan Bretschneider, Christian Eggeling, and Stefan W. Hell. „Breaking the Diffraction Barrier in Fluorescence Microscopy by Optical Shelving“. In: PRL 98 (2007), pp. 218103-1 -218103-4 (cit. on p. 21).

[19]Joshua A. Broussard, Benjamin Rappaz, Donna J. Webb, and Claire M. Brown. „Fluorescence resonance energy transfer microscopy as demonstrated by measuring the activation of the serine/threonine kinase Akt“. In: Nature Protocols 8.2 (2013), pp. 265-281 (cit. on p. 34).

[20]Fiona T. S. Chan, Clemens F. Kaminski, and Gabriele S. Kaminski Schierle. „HomoFRET Fluorescence Anisotropy Imaging as a Tool to Study Molecular Self-Assembly in Live Cells“. In: ChemPhysChem 12.3 (2011), pp. 500-509 (cit. on p. 30).

[21]I. C. Chang. „Acousto-optic tunable filters“. In: Optical Engineering 20.6 (1981), pp. 824 829 (cit. on pp. 176, 177).

[22]Danielle M. Charron and Gang Zheng. „Nanomedicine development guided by FRET imaging“. In: Nano Today (2018) (cit. on p. 34).

[23]WeiYue Chen, Edward Avezov, Simon C. Schlachter, et al. „A Method to Quantify FRET Stoichiometry with Phasor Plot Analysis and Acceptor Lifetime Ingrowth“. In: Biophysical Journal 108.5 (2015), pp. 999-1002 (cit. on pp. 49, 167).

[24]Andriy Chmyrov, Jan Keller, Tim Grotjohann, et al. „Nanoscopy with more than 100,000 'doughnuts'،. In: Nature Methods 10 (2013), pp. 737-740 (cit. on p. 170).

[25]Sangyeon Cho, Jaeduck Jang, Chaeyeon Song, et al. „Simple super-resolution live-cell imaging based on diffusion-assisted Förster resonance energy transfer". In: Scientific Reports 3 (2013), pp. 1208-1 -1208-7 (cit. on p. 39). 
[26]Meir Cohen-Kashi, Yaniv Namer, and Mordechai Deutsch. „Fluorescence resonance energy transfer imaging via fluorescence polarization measurement". In: Journal of Biomedical Optics 11 (2006), pp. $11-11-13$ (cit. on p. 30).

[27]Daniel E. Conway, Mark T. Breckenridge, Elizabeth Hinde, et al. „Fluid Shear Stress on Endothelial Cells Modulates Mechanical Tension across VE-Cadherin and PECAM-1“. In: Current Biology 23.11 (2013), pp. 1024-1030 (cit. on p. 35).

[28]Megan Currie, Hannah Leopold, Jacob Schwarz, et al. „Fluorescence Dynamics of a FRET Probe Designed for Crowding Studies“. In: The Journal of Physical Chemistry B 121.23 (2017), pp. 5688-5698 (cit. on p. 35).

[29]Johann G. Danzl, Sven C. Sidenstein, Carola Gregor, et al. „Coordinate-targeted fluorescence nanoscopy with multiple off states“. In: Nature Photonics (2016) (cit. on p. 21).

[30]Suhui Deng, Jianfang Chen, Zhaoshuai Gao, et al. „Effects of donor and acceptor's fluorescence lifetimes on the method of applying Förster resonance energy transfer in STED microscopy“. In: Journal of Microscopy (2017), pp. 1-7 (cit. on pp. 40, 47).

[31]Suhui Deng, Jianfang Chen, Qing Huang, Chunhai Fan, and Ya Cheng. „Saturated Förster resonance energy transfer microscopy with a stimulated emission depletion beam: a pathway toward single-molecule resolution in far-field bioimaging“. In: Optics Letters 35.23 (2010), pp. 3862-3864 (cit. on p. 40).

[32]Evgenia Dobrinskikh, Luca Lanzano, Joanna Rachelson, et al. „Shank2 contributes to the apical retention and intracellular redistribution of NaPiIIa in OK cells". In: Am J Physiol Cell Physiol 304.6 (2013), pp. C561-C573 (cit. on p. 34).

[33]Christian Eggeling, Katrin I. Willig, Steffen J. Sahl, and Stefan W. Hell. „Lens-based fluorescence nanoscopy“. In: Quarterly Reviews of Biophysics 48 (2015), pp. 178-243 (cit. on pp. 21, 24).

[34]Thomas Elsaesser and Wolfgang Kaiser. „Vibrational and vibronic relaxation of large polyatomic molecules in liquids“. In: Annual review of physical chemistry 42.1 (1991), pp. 83-107 (cit. on p. 135).

[35]Kenneth N. Fish. „Total Internal Reflection Fluorescence (TIRF) Microscopy“. In: Current Protocols in Cytometry. John Wiley \& Sons, Inc., 2001 (cit. on p. 24).

[36]Kazuto Fukuhara, Atsuko Tsurumi, and Masahiro Kotani. „Real-time observation of reversible spectral change of single pyridine 2 molecules adsorbed on a glass surface“. In: Chemical Physics Letters 465.1 (2008), pp. 53-56 (cit. on pp. 13, 135).

[37]Theodorus W. J. Gadella, Arie van Hoek, and Antonie J. W. G. Visser. „Construction and characterization of a frequency-domain fluorescence lifetime imaging microscopy system“. In: J Fluoresc 7.1 (1997), pp. 35-43 (cit. on p. 30).

[38]Silvia Galiani, Benjamin Harke, Giuseppe Vicidomini, et al. „Strategies to maximize the performance of a STED microscope“. In: Opt. Express 20.7 (2012), pp. $7362-7374$ (cit. on p. 23).

[39]F. Goerlitz, P. Hoyer, H. J. Falk, et al. „A STED Microscope Designed for Routine Biomedical Applications“. In: Progress In Electromagnetics Research 147 (2014), pp. 57-68 (cit. on p. 75).

[40]Hernán E. Grecco and Peter J. Verveer. „FRET in Cell Biology: Still Shining in the Age of Super-Resolution?"In: ChemPhysChem 12 (2011), pp. 484-490 (cit. on pp. 37, 156). 
[41]Ingo Gregor and Matthias Patting. „Pattern-Based Linear Unmixing for Efficient and Reliable Analysis of Multicomponent TCSPC Data“. In: Advanced Photon Counting: Applications, Methods, Instrumentation. Ed. by Peter Kapusta, Michael Wahl, and Rainer Erdmann. Cham: Springer International Publishing, 2015, pp. 241-263 (cit. on pp. 115, $167,168)$.

[42]Tim Grotjohann, Ilaria Testa, Marcel Leutenegger, et al. „Diffraction-unlimited all-optical imaging and writing with a photochromic GFP“. In: Nature 478 (2011), p. 204 (cit. on p. 21).

[43]Fabian Göttfert, Christian A. Wurm, Veronika Mueller, et al. „Coaligned Dual-Channel STED Nanoscopy and Molecular Diffusion Analysis at $20 \mathrm{~nm}$ Resolution“. In: Biophysical Journal 105.1 (2013), pp. L01-L03 (cit. on p. 21).

[44]T. Ha, Th. Enderle, D. S. Chemla, P. R. Selvin, and S. Weiss. „Quantum jumps of single molecules at room temperature“. In: Chemical Physics Letters 271.1 (1997), pp. 1-5 (cit. on p. 13).

[45]Taekjip Ha and Philip Tinnefeld. „Photophysics of Fluorescent Probes for Single-Molecule Biophysics and Super-Resolution Imaging“. In: Annu. Rev. Phys. Chem. 63 (2012), pp. 595617 (cit. on p. 12).

[46]Handbook of optics. McGraw-Hill, 1995 (cit. on pp. 15, 16).

[47] G. S. Harms, L. Cognet, P. H. Lommerse, G. A. Blab, and T. Schmidt. „Autofluorescent proteins in single-molecule research: applications to live cell imaging microscopy“. In: Biophys J 80.5 (2001), pp. 2396-408 (cit. on p. 160).

[48]Elke Hebisch. „STED microscopy of cardiac membrane nanodomains“. Thesis. 2017 (cit. on p. 33).

[49]Stefan W. Hell. „Far-Field Optical Nanoscopy“. In: Science 316.5828 (2007), pp. 11531158. eprint: http://science.sciencemag.org/content/316/5828/1153.full.pdf (cit. on pp. 22, 23).

[50]Stefan W. Hell, Steffen J. Sahl, Mark Bates, et al. „The 2015 super-resolution microscopy roadmap“. In: J. Phys. D: Appl. Phys. 48 (2015) (cit. on p. 24).

[51]Kari A. Herrington, Andrew L. Trinh, Carolyn Dang, et al. „Spatial analysis of Cdc42 activity reveals a role for plasma membrane-associated Cdc42 in centrosome regulation". In: $M B o C$ (2017) (cit. on p. 34).

[52]Liisa M. Hirvonen and Klaus Suhling. „Wide-field TCSPC: methods and applications“. In: Meas. Sci. Technol. 28 (2016), pp. 012003-1 -012003-19 (cit. on pp. 29, 155).

[53]Pekka E. Hänninen, Lotta Lehtelä, and Stefan W. Hell. „Two- and multiphoton excitation of conjugate-dyes using a continuous wave laser“. In: Optics Communications 130.1 (1996), pp. 29-33 (cit. on p. 40).

[54]Bernhard Hochreiter, Alan Pardo Garcia, and Johannes A. Schmid. „Fluorescent Proteins as Genetically Encoded FRET Biosensors in Life Sciences“. In: Sensors 15 (2015), pp. 26281-26314 (cit. on pp. 27, 34, 35, 44, 159).

[55]Phil Holzmeister, Andreas Gietl, and Philip Tinnefeld. „Geminate Recombination as a Photoprotection Mechanism for Fluorescent Dyes“. In: Angew. Chem. Int. Ed. 53 (2014), pp. 5685-5688 (cit. on p. 87). 
[56]Phil Holzmeister, Bettina Wünsch, Andreas Gietl, and Philip Tinnefeld. „Single-molecule photophysics of dark quenchers as non-fluorescent FRET acceptors". In: Photochem. Photobiol. Sci. 13.6 (2014), pp. 853-858 (cit. on pp. 39, 156).

[57]Kazuyuki Horie, Hideharu Ushiki, et al. Molecular photonics: fundamentals and practical aspects. John Wiley \& Sons, 2000 (cit. on p. 14).

[58]Robert G Hunsperger. „Acousto-optic modulators“. In: Integrated Optics. Springer, 2009, pp. 201-220 (cit. on p. 175).

[59]Peter Ilgen, Stefan Stoldt, Lena-Christin Conradi, et al. „STED Super-Resolution Microscopy of Clinical Paraffin-Embedded Human Rectal Cancer Tissue“. In: PLOS ONE 9.7 (July 2014), pp. 1-8 (cit. on p. 34).

[60]Joseph M. Johnson, Taekjip Ha, Steve Chu, and Steven G. Boxer. „Early Steps of Supported Bilayer Formation Probed by Single Vesicle Fluorescence Assays". In: Biophysical Journal 83.6 (2002), pp. 3371 -3379 (cit. on p. 14).

[61]Chirlmin Joo, Sean A. McKinney, Muneaki Nakamura, et al. „Real-Time Observation of RecA Filament Dynamics with Single Monomer Resolution“. In: Cell 126 (2006), pp. 515527 (cit. on p. 34).

[62]Manuel F. Juette, Daniel S. Terry, Michael R. Wasserman, et al. „Single-molecule imaging of non-equilibrium molecular ensembles on the millisecond timescale“. In: Nat Meth 13.4 (2016), pp. 341-344 (cit. on p. 34).

[63]Bryan Kaye, Peter J. Foster, Tae Yeon Yoo, and Daniel J. Needleman. „Developing and Testing a Bayesian Analysis of Fluorescence Lifetime Measurements“. In: PLoS ONE 12.1 (2017), e0169337-1-e0169337-13 (cit. on p. 168).

[64]Christian Kempf, Thorsten Staudt, Pit Bingen, et al. „Tissue Multicolor STED Nanoscopy of Presynaptic Proteins in the Calyx of Held“. In: PLOS ONE 8.4 (Apr. 2013), pp. 1-10 (cit. on p. 33).

[65]R. Kettner, J. Tittel, Th. Basche, and C. Braeuchle. „Optical Spectroscopy and Spectral Diffusion of Single Dye Molecules in Amorphous Spin-Coated Polymer Films“. In: The Journal of Physical Chemistry 98.27 (1994), pp. 6671-6674 (cit. on p. 13).

[66]Doory Kim, Zhengyang Zhang, and Ke Xu. „Spectrally Resolved Super-Resolution Microscopy Unveils Multipath Reaction Pathways of Single Spiropyran Molecules“. In: Journal of the American Chemical Society 139.28 (2017), pp. 9447-9450 (cit. on p. 135).

[67]Petr Klán and Jakob Wirz. Photochemistry of organic compounds: from concepts to practice. John Wiley \& Sons, 2009 (cit. on pp. 11, 14).

[68]Andrey S. Klymchenko. „Solvatochromic and Fluorogenic Dyes as Environment-Sensitive Probes: Design and Biological Applications“. In: Accounts of Chemical Research 50.2 (2017), pp. 366-375 (cit. on p. 13).

[69]Kirill Kolmakov, Vladimir N. Belov, Christian A. Wurm, et al. „A Versatile Route to RedEmitting Carbopyronine Dyes for Optical Microscopy and Nanoscopy“. In: European Journal of Organic Chemistry 2010.19 (2010), pp. 3593-3610 (cit. on p. 97).

[70]Sheldon J. J. Kwok, Myunghwan Choi, Brijesh Bhayana, et al. „Two-photon excited photoconversion of cyanine-based dyes“. In: Scientific Reports 6 (2016), p. 23866 (cit. on p. 14). 
[71]Joseph R. Lakowicz. Principles of Fluorescence Spectroscopy. 3rd. New York: Springer Science+Business Media, LLC, 2006 (cit. on p. 25).

[72]Ludovic Le Reste, Johannes Hohlbein, Kristofer Gryte, and Achillefs N. Kapanidis. „Characterization of Dark Quencher Chromophores as Nonfluorescent Acceptors for SingleMolecule FRET“. In: Biophysical Journal 102.11 (2012), 2658-2668 (cit. on pp. 85, 169).

[73]Marcel Leutenegger, Christian Eggeling, and Stefan W. Hell. „Analytical description of STED microscopy performance“. In: Optics Express 18.25 (2010), pp. 26417-26429 (cit. on p. 52).

[74]Wen-hong Li and Genhua Zheng. „Photoactivatable fluorophores and techniques for biological imaging applications“. In: Photochemical \& photobiological sciences : Official journal of the European Photochemistry Association and the European Society for Photobiology 11.3 (2012), pp. 460-471 (cit. on p. 14).

[75]Zhiyu Liao, Sidsel Ammitzbøll Bogh, Marco Santella, et al. „Emissive Photoconversion Products of an Amino-triangulenium Dye“. In: The Journal of Physical Chemistry A 120.20 (2016), pp. 3554-3561 (cit. on p. 14).

[76]Zhiyu Liao, Emma N. Hooley, Long Chen, et al. „Green Emitting Photoproducts from Terrylene Diimide after Red Illumination". In: Journal of the American Chemical Society 135.51 (2013). PMID: 24304094, pp. 19180-19185. eprint: http://dx.doi .org/10. 1021/ja407431w (cit. on p. 14).

[77]Sebastian van de Linde and Markus Sauer. „How to switch a fluorophore: from undesired blinking to controlled photoswitching“. In: Chem. Soc. Rev. 43 (2014), pp. 1076-1087 (cit. on p. 12).

[78]Stephen G. Lipson, Henry S. Lipson, and D.S. Tannhauser. Optik. Springer-Lehrbuch. Springer-Verlag Berlin Heidelberg, 1997, p. 490 (cit. on p. 16).

[79]Y. Liu, D.K. Cheng, G.J. Sonek, et al. „Evidence for localized cell heating induced by infrared optical tweezers“. In: Biophysical Journal 68.5 (1995), pp. 2137 -2144 (cit. on p. 135).

[80]Maria Loidolt. „Mehrfarben-STED-Mikroskopie“. Thesis. 2013 (cit. on pp. 81, 207).

[81]Anastasia Loman, Ingo Gregor, Christina Stutz, Markus Mund, and Jörg Enderlein. „Measuring rotational diffusion of macromolecules by fluorescence correlation spectroscopy“. In: Photochem. Photobiol. Sci. 9 (2010), pp. 627-636 (cit. on p. 160).

[82]H. Peter Lu and X. Sunney Xie. „Single-molecule spectral fluctuations at room temperature“. In: Nature 385 (1997), p. 143 (cit. on p. 13).

[83]Boris Martinac. „Single-molecule FRET studies of ion channels“. In: Progress in Biophysics and Molecular Biology (2017) (cit. on p. 34).

[84]Igor L. Medintz and Niko Hildebrandt. FRET-Förster resonance energy transfer: from theory to applications. John Wiley \& Sons, 2013 (cit. on pp. 25, 26, 30, 35).

[85]Sohum Mehta and Jin Zhang. „Illuminating the cell's biochemical activity architecture“. In: ACS Biochemistry (2017) (cit. on p. 35).

[86]Karl Dieter Möller. Optics. 2nd ed. Springer-Verlag New York, 2007, pp. XVI, 455 (cit. on p. 9). 
[87]Masayuki X. Mori, Yuko Imai, Kyohei Itsuki, and Ryuji Inoue. „Quantitative Measurement of Ca2+-Dependent Calmodulin-Target Binding by Fura-2 and CFP and YFP FRET Imaging in Living Cells“. In: Biochemistry 50 (2011), pp. 4685-4696 (cit. on p. 34).

[88]Douglas B. Murphy and Michael W. Davidson. „Frontmatter“. In: Fundamentals of Light Microscopy and Electronic Imaging. John Wiley \& Sons, Inc., 2012, pp. i-xiii (cit. on p. 8).

[89]Thomas Niehörster, Anna Löschberger, Ingo Gregor, et al. „Multi-target spectrally resolved fluorescence lifetime imaging microscopy“. In: Nature Methods 13.3 (2016), pp. 257-262 (cit. on pp. 115, 167, 168).

[90]Takashi Ohtsuki, Shunya Miki, Shouhei Kobayashi, et al. „The molecular mechanism of photochemical internalization of cell penetrating peptide-cargo-photosensitizer conjugates“. In: Scientific Reports 5 (2015), p. 18577 (cit. on p. 96).

[91]Joanna Oracz, Volker Westphal, Czesław Radzewicz, Steffen J. Sahl, and Stefan W. Hell. „Photobleaching in STED nanoscopy and its dependence on the photon flux applied for reversible silencing of the fluorophore“. In: Scientific Reports 7.1 (2017), p. 11354 (cit. on pp. 13, 69, 93, 97, 98, 168).

[92]Sandra Orthaus-Mueller, Ben Kraemer, Rhys Dowler, et al. rapidFLIM: The New and Innovative Method for Ultra fast FLIM Imaging. Application Note. PicoQuant GmbH, 2016 (cit. on p. 29).

[93]Christian Osseforth, Jeffrey R. Moffitt, Lothar Schermelleh, and Jens Michaelis. „Simultaneous dual-color 3D STED microscopy“. In: Opt. Express 22.6 (2014), pp. 7028-7039 (cit. on p. 1).

[94]Sergi Padilla-Parra and Marc Tramier. „FRET microscopy in the living cell: Different approaches, strengths and weaknesses“. In: Bioessays 34 (2012), pp. 369-376 (cit. on pp. 25, 159).

[96]Megha Rajendran and Lawrence W. Miller. „Evaluating the Performance of Time-Gated Live-Cell Microscopy with Lanthanide Probes“. In: Biophysical Journal 109.2 (2015), pp. 240-248 (cit. on p. 42).

[97]Megha Rajendran, Engin Yapici, and Lawrence W. Miller. „Lanthanide-Based Imaging of Protein-Protein Interactions in Live Cells“. In: Inorg. Chem. 53.4 (2013), pp. 1839-1853 (cit. on p. 42).

[98]Malte Renz, Brian R. Daniels, György Vámosi, Irwin M. Arias, and Jennifer LippincottSchwartz. „Plasticity of the asialoglycoprotein receptor deciphered by ensemble FRET imaging and single-molecule counting PALM imaging“. In: PNAS 109.44 (2012), E2989-E2997 (cit. on pp. 34, 41).

[99]Douglas S. Richardson, Carola Gregor, Franziska R. Winter, et al. „SRpHi ratiometric $\mathrm{pH}$ biosensors for super-resolution microscopy“. In: Nature Communications 8 (2017), p. 577 (cit. on p. 34).

[100]Rahul Roy, Sungchul Hohng, and Taekjip Ha. „A practical guide to single-molecule FRET“. In: Nature Methods 5.6 (2008), pp. 507-516 (cit. on p. 34).

[101]Steffen J. Sahl, Stefan W. Hell, and Stefan Jakobs. „Fluorescence nanoscopy in cell biology“. In: Nat Rev Mol Cell Biol advance online publication (2017) (cit. on p. 33). 
[102]Volkan Sakin, Janina Hanne, Jessica Dunder, et al. „A Versatile Tool for Live-Cell Imaging and Super-Resolution Nanoscopy Studies of HIV-1 Env Distribution and Mobility". In: Cell Chemical Biology 24.5 (2017), 635 -645.e5 (cit. on p. 33).

[103]Stefan Scharring, Albrecht Brandenburg, Gernot Breitfuss, et al. „Online Monitoring of Airborne Allergenic Particles (OMNIBUSS)“. In: Biophotonics. Wiley-VCH Verlag GmbH \& Co. KGaA, 2006, pp. 31-87 (cit. on p. 19).

[104]Andreas Schönle, Pekka E. Hänninen, and Stefan W. Hell. „Nonlinear fluorescence through intermolecular energy transfer and resolution increase in fluorescence microscopy". In: Annalen der Physik 8.2 (1999), pp. 115-134 (cit. on p. 40).

[105]Andreas Schönle and Stefan W. Hell. „Heating by absorption in the focus of an objective lens“. In: Opt. Lett. 23.5 (1998), pp. 325-327 (cit. on p. 135).

[106]G. Schreiber, G. Haran, and H. X. Zhou. „Fundamental aspects of protein-protein association kinetics“. In: Chem Rev 109.3 (2009), pp. 839-60 (cit. on p. 160).

[107]Benjamin Schuler and William A. Eaton. „Protein folding studied by single-molecule FRET“. In: Current Opinion in Structural Biology 18.1 (2008). Folding and Binding / Protein-nucleic acid interactions, pp. 16-26 (cit. on p. 34).

[108]Science of Microscopy. Springer-Verlag New York, 2007, p. 1322 (cit. on pp. 17, 18).

[109]Alexey Sharonov and Robin M. Hochstrasser. „Wide-field subdiffraction imaging by accumulated binding of diffusing probes". In: Proceedings of the National Academy of Sciences 103.50 (2006), pp. 18911-18916. eprint: http://www . pnas.org/content/103/ 50/18911.full.pdf (cit. on p. 24).

[110]Dilip Shrestha, Attila Jenei, Péter Nagy, György Vereb, and János Szöllősi. „Understanding FRET as a Research Tool for Cellular Studies". In: International Journal of Molecular Sciences 16.4 (2015), p. 6718 (cit. on pp. 25, 28).

[111]Sven C. Sidenstein, Elisa D'Este, Marvin J. Böhm, et al. „Multicolour Multilevel STED nanoscopy of Actin/Spectrin Organization at Synapses“. In: Scientific Reports 6 (2016), pp. 26725-1 -26725-8 (cit. on p. 33).

[112]Elana M. S. Stennett, Monika A. Ciuba, and Marcia Levitus. „Photophysical processes in single molecule organic fluorescent probes“. In: Chemical Society Reviews 43.4 (2014), pp. 1057-1075 (cit. on p. 136).

[113]Rainer J. Stoehr, Roman Kolesov, Kangwei Xia, et al. „Super-resolution Fluorescence Quenching Microscopy of Graphene“. In: ASC Nano 6.10 (2012), pp. 9175-9181 (cit. on pp. 41, 42).

[114]Rita Strack. „Taking nanoscopy to the limit“. In: Nature Methods 14 (2017), p. 221 (cit. on p. 24).

[115]Frank Stracke, Christian Blum, Stefan Becker, Klaus Müllen, and Alfred J. Meixner. "Correlation of Emission Intensity and Spectral Diffusion in Room Temperature SingleMolecule Spectroscopy“. In: ChemPhysChem 6.7 (2005), pp. 1242-1246 (cit. on p. 13).

[116] Sune Svanberg. Atomic and molecular spectroscopy: basic aspects and practical applications. Vol. 6. Springer Science \& Business Media, 2012 (cit. on pp. 7, 9).

[117]Mariusz Szabelski, Rafal Luchowski, Zygmunt Gryczynski, et al. „Evaluation of instrument response functions for lifetime imaging detectors using quenched Rose Bengal solutions". In: Chemical Physics Letters 471 (2009), pp. 153-159 (cit. on pp. 29, 88, 99). 
[118]Johnny Tam and David Merino. „Stochastic optical reconstruction microscopy (STORM) in comparison with stimulated emission depletion (STED) and other imaging methods“. In: Journal of Neurochemistry 135.4 (2015), pp. 643-658 (cit. on p. 1).

[119]Ilaria Testa, Elisa D’Este, Nicolai T. Urban, Francisco Balzarotti, and Stefan W. Hell. „Dual Channel RESOLFT Nanoscopy by Using Fluorescent State Kinetics“. In: Nano Lett. 15.1 (2015), pp. 103-106 (cit. on p. 170).

[120]Philip Tinnefeld, Dirk-Peter Herten, and Markus Sauer. „Photophysical Dynamics of Single Molecules Studied by Spectrally-Resolved Fluorescence Lifetime Imaging Microscopy (SFLIM)“. In: The Journal of Physical Chemistry A 105.34 (2001), pp. 7989-8003 (cit. on p. 13).

[121]Giorgio Tortarolo, Marco Castello, Alberto Diaspro, Sami Koho, and Giuseppe Vicidomini. „Evaluating image resolution in stimulated emission depletion microscopy“. In: Optica 5.1 (2018), pp. 32-35 (cit. on p. 17).

[122]J. K. Trautman, J. J. Macklin, L. E. Brus, and E. Betzig. „Near-field spectroscopy of single molecules at room temperature“. In: Nature 369 (1994), p. 40 (cit. on p. 13).

[123]Roger Y. Tsien and Alan Waggoner. „Fluorophores for confocal microscopy“. In: Handbook of biological confocal microscopy. Springer, 1995, pp. 267-279 (cit. on p. 160).

[124]David Unnersjö-Jess, Lena Scott, Hans Blom, and Hjalmar Brismar. „Super-resolution stimulated emission depletion imaging of slit diaphragm proteins in optically cleared kidney tissue“. In: Kidney International 89.1 (2016), pp. 243 -247 (cit. on p. 34).

[125]Nicolai T. Urban, Katrin I. Willig, Stefan W. Hell, and U. Valentin Nägerl. „STED Nanoscopy of Actin Dynamics in Synapses Deep Inside Living Brain Slices“. In: Biophysical Journal 101.5 (2011), pp. $1277-1284$ (cit. on p. 33).

[126]Bernard Valeur and Mario Nuno Berberan-Santos. Molecular Fluorescence: Principles and Applications. Wiley-VCH, 2012, p. 592 (cit. on pp. 5, 7, 11, 18, 25, 26, 64).

[127]Darren B. VanBeek, Matthew C. Zwier, Justin M. Shorb, and Brent P. Krueger. „Fretting about FRET: Correlation between k and R“. In: Biophysical Journal 92 (2007), pp. 41684178 (cit. on p. 160).

[128]Giuseppe Vicidomini, Ivan Coto Hernández, Marta d'Amora, et al. „Gated CW-STED microscopy: A versatile tool for biological nanometer scale investigation“. In: Methods 66.2 (2014), pp. 124-130 (cit. on pp. 47, 69).

[129]Giuseppe Vicidomini, Gael Moneron, Christian Eggeling, Eva Rittweger, and Stefan W. Hell. „STED with wavelengths closer to the emission maximum“. In: Optics Express 20.5 (2012), pp. 5225-5236 (cit. on p. 40).

[130]Jan Vogelsang, Robert Kasper, Christian Steinhauer, et al. „A Reducing and Oxidizing System Minimizes Photobleaching and Blinking of Fluorescent Dyes“. In: Angew. Chem. Int. Ed. 47 (2008), pp. 5465-5469 (cit. on pp. 12, 87).

[131]Michael Wahl. Time-Correlated Single Photon Counting. Technical Note. PicoQuant GmbH, 2014 (cit. on p. 29).

[132]Tadashi Watanabe, Takuo Takizawa, and Kenichi Honda. „Photocatalysis through excitation of adsorbates. 1. Highly efficient N-deethylation of rhodamine B adsorbed to cadmium sulfide“. In: The Journal of Physical Chemistry 81.19 (1977), pp. 1845-1851 (cit. on p. 14). 
[133]Waja Wegner, Alexander C. Mott, Seth G. N. Grant, Heinz Steffens, and Katrin I. Willig. „In vivo STED microscopy visualizes PSD95 sub-structures and morphological changes over several hours in the mouse visual cortex“. In: Scientific Reports 8.1 (2018), p. 219 (cit. on p. 34).

[134] Christopher M. Welch, Hunter Elliott, Gaudenz Danuser, and Klaus M. Hahn. „Imaging the coordination of multiple signalling activities in living cells“. In: Nature Reviews Molecular Cell Biology 12 (2011), pp. 749-756 (cit. on p. 35).

[135]Jerker Widengren, Andriy Chmyrov, Christian Eggeling, Per-Åke Löfdahl, and Claus A. M. Seidel. „Strategies to Improve Photostabilities in Ultrasensitive Fluorescence Spectroscopy“. In: The Journal of Physical Chemistry A 111.3 (2007). PMID: 17228891, pp. 429440. eprint: http://dx.doi.org/10.1021/jp0646325 (cit. on p. 13).

[136]Martin Wiklund, Hjalmar Brismar, and Bjorn Onfelt. „Acoustofluidics 18: Microscopy for acoustofluidic micro-devices“. In: Lab Chip 12 (18 2012), pp. 3221-3234 (cit. on p. 18).

[137]Dominik Wildanger, Johanna Bückers, Volker Westphal, Stefan W. Hell, and Lars Kastrup. „A STED microscope aligned by design“. In: Opt. Express 17.18 (2009), pp. 16100-16110 (cit. on p. 23).

[138] Katrin I. Willig, Heinz Steffens, Carola Gregor, et al. „Nanoscopy of Filamentous Actin in Cortical Dendrites of a Living Mouse“. In: Biophysical Journal 106.1 (2014), pp. L01-L03 (cit. on p. 34).

[139]Katrin I. Willig, Benjamin Harke, Rebecca Medda, and Stefan W. Hell. „STED microscopy with continuous wave beams“. In: Nat Meth 4.11 (2007), pp. 915-918 (cit. on p. 52).

[140]Pascale Winckler, Lydia Lartigue, Gregory Giannone, et al. „Identification and superresolution imaging of ligand-activated receptor dimers in live cells". In: Scientific Reports 3 (2013), pp. 2387-1 -2387-5 (cit. on pp. 34, 41).

[141]Franziska R. Winter, Maria Loidolt, Volker Westphal, et al. „Multicolour nanoscopy of fixed and living cells with a single STED beam and hyperspectral detection". In: Scientific Reports 7 (2017), p. 46492 (cit. on p. 33).

[142]Franziska Ruth Winter. „Multicolour STED nanoscopy with hyperspectral detection“. Thesis. 2016 (cit. on pp. 73-76, 96, 97, 119, 136, 138).

[143]Qian Wu, Miao Sun, Laura P. Bernard, and Huaye Zhang. „Postsynaptic density 95 (PSD95) serine 561 phosphorylation regulates a conformational switch and bidirectional dendritic spine structural plasticity“. In: J. Biol. Chem. (2017) (cit. on p. 34).

[144]Christian A. Wurm, Kirill Kolmakov, Fabian Göttfert, et al. „Novel red fluorophores with superior performance in STED microscopy“. In: Optical Nanoscopy 1.1 (2012), p. 7 (cit. on pp. 137, 185).

[145]X. Sunney Xie and Robert C. Dunn. „Probing Single Molecule Dynamics“. In: Science 265.5170 (1994), pp. 361-364. eprint: http://science. sciencemag.org/content/ 265/5170/361.full.pdf (cit. on p. 13).

[146]Alsu I. Zamaleeva, Guillaume Despras, Camilla Luccardini, et al. „FRET-Based Nanobiosensors for Imaging Intracellular Ca2+ and H+ Microdomains“. In: Sensors 15 (2015), pp. $24662-24680$ (cit. on p. 35 ). 
[147]André Zeug, Andrew Woehler, Erwin Neher, and Evgeni G. Ponimaskin. „Quantitative Intensity-Based FRET Approaches—A Comparative Snapshot“. In: Biophysical Journal 103.9 (2012), pp. 1821-1827 (cit. on p. 27).

[148]Xi Zhang, Mingshu Zhang, Dong Li, et al. „Highly photostable, reversibly photoswitchable fluorescent protein with high contrast ratio for live-cell superresolution microscopy". In: Proceedings of the National Academy of Sciences of the United States of America 113.37 (2016), pp. 10364-10369 (cit. on p. 156).

[149]Zhengyang Zhang, Samuel J. Kenny, Margaret Hauser, Wan Li, and Ke Xu. „Ultrahighthroughput single-molecule spectroscopy and spectrally resolved super-resolution microscopy“. In: Nat Meth 12.10 (2015), pp. 935-938 (cit. on p. 135).

[150]Shenfei Zong, Xiaoyue Jiang, Zhuyuan Wang, et al. „FRET Based Dual Emission Nanoprobe (FREDEN) with Improved Blinking Behavior for Single Molecule Localization Imaging“. In: Nanoscale (2016), pp. 1-27 (cit. on p. 39).

\section{Webseiten}

[95]porphyrin. Why are the absorption and emission spectra mirror images of each other? 2016. URL: https : // chemistry . stackexchange . com/questions/55574/why-arethe-absorption-and-emission-spectra-mirror-images-of-each-other (visited on Dec. 25, 2017) (cit. on p. 7). 


\section{List of Figures}

2.1 Instantaneous (vertical) transitions, according to the Franck-Condon principle, between different vibrational levels of ground and excited state (potential energy curves of nuclear configurations), give rise to the shape of absorption and emission spectra of fluorophores. Taken from [95]. . . . 7

2.2 Normalized absorption and fluorescence emission spectra of fluoresceinconjugated immunoglobulin $\mathrm{G}$ antibodies. Taken from [88] . . . . . . . 8

2.3 Jablonski diagram depicting a molecule's electronic states and transitions between them. Adapted from [88]. . . . . . . . . . . . . . 8

2.4 Schematic of stimulated absorption and stimulated and spontaneous emission. Adapted from $[86] . \ldots \ldots$. . . . . . . . . . . . . 9

4.1 Ray path in the microscope from the object to the observer's eye. Taken from [46]. . . . . . . . . . . . . . . . . . . . . 15

4.2 Ray path in a microscope with infinity-corrected objective and tube lens. Taken from $[46] . \ldots \ldots \ldots \ldots \ldots \ldots \ldots$

4.3 Conceptual-model illustration of the Rayleigh criterion for the minimum resolvable distance D between two small point-like objects. (a) The diffraction-limited predicted image (lower panel) of a small object (upper panel) consisting of a bright sphere. (b) The corresponding image of two such small objects, unresolved case. (c) Same as in (b) but the limit where the spacing, $\mathrm{D}$, is just large enough for resolving the two small spheres. The radius of the imaged spot, $r_{\text {spot }}$, in (a) is equal to $\mathrm{D}$ in (c). The situation in (c) illustrates the Rayleigh criterion. Adapted from [136]. . . . . . . . . 18

4.4 Working principle of the fluorescence microscope, taken from [103] . . . 19

4.5 The confocal pinhole rejects light from out-of-focus planes. . . . . . . . . . 19

5.1 STED mode of operation: The diffraction-limited excitation focus excites the fluorophores. The torus-shaped STED focus transfers the fluorophores in the periphery of the excitation spot back to the non-fluorescent ground state. The remaining fluorophores in the center of the STED doughnutfocus are allowed to emit fluorescence. Taken from [1] . . . . . . . . . .

5.2 Coordinate-targeted read-out with STED: Excitation focus (blue) and STED focus (orange) are superimposed and scanned across the sample simultaneously. Only fluorophores in the effective excitation spot (green) emit fluorescence, as illustrated in Fig. 5.1. Thus fluorophores spaced closer than the diffraction limit are read out sequentially to obtain a diffractionunlimited image. Adapted from [49]. . . . . . . . . . . . . . . 
5.3 A vortex phase plate (left) induces a helical phase shift in the circularly polarized STED beam, which creates a doughnut-shaped focus (right) by destructive interference. Taken from [137] . . . . . . . . . . .

5.4 Simulated effective STED PSF for different incomplete inhibition factor $\delta$. Incomplete depletion was modeled by adding the constant offset $\delta$ in the spontaneous emission probability. Adapted from [38]. . . . . . . . . .

9.1 Jablonski diagram showing electronic transitions between ground and first excited state upon absorption, spontaneous and stimulated emission, for a single fluorophore and for two fluorophores between which FRET occurs. Figure adapted from $[54] . \ldots \ldots \ldots \ldots$. . . . . . . . .

10.1 Top: Simulations of the excited state population over time, i.e. lifetime histograms, of a donor and acceptor with different transfer rates under CW-STED light of constant intensity. (semi-log plots) Bottom: Line profiles through the center of the spatial distribution of the excited state populations. The legend states the lifetime of the donor in the presence of the acceptor in ns, refer to table 10.1. The data in the right plots is the same as in the left plots, only normalized. . . . . . . . . . . . .

10.2 Top half: Simulation of the excited state dynamics of a FRET pair with 60\% transfer efficiency under CW-STED with increasing intensity. (semi-log plots) Bottom half: Simulated line profiles of the same FRET pair. The legend states the STED peak intensity in arbitrary units. The data on the right hand side is the same as in the left plots, only normalized. . . . . . .

11.1 Top half: Simulation of the excited state dynamics of FRET pairs with different transfer rates under pulsed STED light of constant intensity. (semi-log plots) Bottom half: Simulated line profiles of FRET pairs with different transfer rates. The legends state the lifetime of the donor in ns, as listed in table 10.1. The histograms in the lower panel show the same data as above, only normalized. . . . . . . . . . . . . .

11.2 Simulation of the excited state dynamics of a FRET pair with 69\% transfer efficiency under pulsed STED light of increasing intensity. Top: Simulated lifetime histograms of donor and acceptor. (semi-log plots) Bottom: Simulated line profiles. The legend states the maximum STED intensity in arbitrary units. . . . . . . . . . . . . .

11.3 Simulation of the excited state dynamics of a FRET pair with 69\% transfer efficiency under pulsed STED light with an increasing delay between excitation and STED pulse. Top: Simulated lifetime histograms of donor and acceptor. (semi-log plots) Bottom: Simulated line profiles. The legend states the arrival time of the STED pulse in ns. The excitation pulse arrives

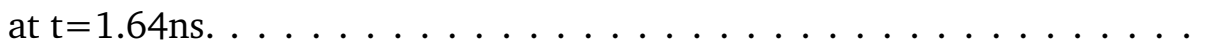


11.4 Simulation of the excited state dynamics of FRET pairs with different transfer rates under pulsed STED light of constant intensity. The borders of the applied time gates 1 to 4 are marked with vertical black lines. The legend states the lifetime of the donor in the presence of the acceptor in ns, refer to table 10.1. . . . . . . . . . . . . . . . . . 61

11.5 Simulated line profiles of FRET pairs with different transfer rates under pulsed STED light of constant intensity. Only photons emitted within the time gates indicated in Fig. 11.4 contributed to the respective line profiles. Left: absolute photon counts. Right: normalized profiles. The legends state the lifetime of the donor fluorophore in ns, refer to table 10.1. . . .

11.6 Simulation of the excited state dynamics of FRET pairs with different transfer rates under pulsed STED light of constant intensity. The borders of the time gates 1 to 4 are marked with vertical black lines. The legend states the lifetime of the donor in the presence of the acceptor in ns, refer to table $11.1 . \ldots \ldots \ldots \ldots \ldots \ldots \ldots \ldots \ldots$

11.7 Simulated line profiles of FRET pairs with different transfer rates under pulsed STED light of constant intensity. Only photons emitted within the stated time gates, indicated in Fig. 11.6, contributed to the respective line profiles. Left: absolute photon counts. Right: normalized profiles. The legends state the lifetime of the donor fluorophore in ns, refer to table 11.1. 65

13.1 Overview of the optical setup. The excitation light is provided by a SC laser, an arbitrary wavelength is selected by an AOBS consisting of three AOTFs. The STED laser power is modulated by an AOM; a vortex phase plate in conjunction with a quarter-wave plate creates the torus-shaped STED focus. Both beams are overlayed by a dichroic mirror and scanned with a quadscanner. The fluorescence light passes the AOBS and is filtered by a confocal pinhole before entering the hyperspectral detection unit. A cascade of longpass filters splits the fluorescence into different spectral bands, each is detected by a separate APD. Figure adapted from [142]. .

13.2 Spectral windows of the seven APDs as defined by the edges of the longpass filters, taking the spectral detection efficiency of the APDs into account. Figure adapted from $[142] \ldots \ldots \ldots \ldots \ldots$

13.3 Electronic control of the setup. Laser trigger pulses were delayed by a variable amount of time by the electronic dual channel delay unit. The FPGA board controlled both scanning and data acquisition. TCSPC was performed with the SPC-150 card from Becker\&Hickl. . . . . . . . . . .

16.1 Sketch of the fluorescently labeled DNA immobilized in the flow channel on the surface of the coverslip by biotin-streptavidin interaction. . . . . . 
17.1 Left: Bleaching rates $k_{\text {bleach }}$ of tested green fluorophores under $775 \mathrm{~nm}$ STED illumination of increasing intensity. Error bars correspond to the error of the single exponential decay fit. Right: Excitation of the green fluorophores by the STED beam whose intensity was increased step by step. The excitation ratio is the ratio of the emission due to the STED beam and the emission due to blue excitation light of constant power. Error bars correspond to the standard deviation of three measurements at the same power. The vortex phase plate was removed from the STED beam for all measurements except those of Atto532 and Atto550 performed on 13th Aug. 2015 . . . . . . . . . . . . . . . . . . .

18.1 Fluorescence decay curves accumulated in donor and FRET channel, i.e. with green excitation in the green or red detection channel, with different STED parameters from several single molecule FRET constructs, detailed in table $15.1 . \ldots \ldots \ldots \ldots \ldots \ldots \ldots \ldots$

18.2 Fluorescence decay curves accumulated in donor and FRET channel for FRET construct DNA79. Top: The STED intensity was varied while keeping the pulse delay constant. Bottom: The STED pulse delay was increased in 320ps steps while keeping the STED intensity constant. The legend states the delay value in ns. All histograms were deconvolved with the measured IRF and normalized. . . . . . . . . . . . . . . . .

19.1 Normalized absorption and emission spectra of Atto532 and Star635P plotted over the spectral windows of detection channels APD4 and APD6. Excitation wavelengths of $532 \mathrm{~nm}$ and $612 \mathrm{~nm}$ as well as the STED wavelength of $775 \mathrm{~nm}$ are indicated. . . . . . . . . . . . . . . . 106

19.2 Determining the FWHM dependence on different STED parameters. Scans of more than 150 small ROIs were centered, overlaid and summed. Weights for fitting were determined according to the overlay, and used to fit the summed images with a 2D Gaussian model function. Resulting FWHM values of the Gaussian fit are plotted in Fig. 19.3. . . . . . . . . . . . 107

19.3 FWHM of a 2D Gaussian function fitted to the summed overlay of many small ROI scans with appropriate weights. Over 150 scans were conducted for each set of STED parameters in each FRET sample. Gray areas: values where fit converged on noise, 300nm was set as upper bound for the STED scan fits. . . . . . . . . . . . . . . . . . . . . . 108

19.4 FWHM calculated from simulations with the stated FRET efficiencies E for different STED powers and pulse delays. These values of $\mathrm{E}$ are the theoretically expected values for DNA constructs 79 to 83. . . . . . . . . 109

20.1 Simulated overlay of donor and acceptor fluorescence images, which differ in spatial resolution. White circles indicate the detection area from which the signal is integrated at different scan positions: a) Signal from one FRET pair. b) Signal from two adjacent FRET pairs. c) Signal from two acceptors and five donors. . . . . . . . . . . . . . . . . . . 111 
20.2 Fluorescence intensity ratios $E_{\mathrm{FRET}}$ and $R_{\mathrm{FRET}}$ were calculated from images of single molecule FRET constructs detailed in table 15.1, which were acquired with different STED powers and pulse delays. Error bars correspond to the s.d. between individual pairs. . . . . . . . . . . . . .

20.3 Fluorescence intensity ratios $E_{\mathrm{FRET}}$ and $R_{\mathrm{FRET}}$ were calculated from simulations of single molecule FRET pairs with the same efficiencies as detailed

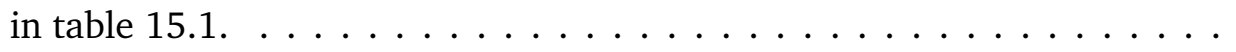

20.4 Scatter plots with the acceptor fluorescence intensity ratio $R_{\text {FRET }}$ of emission caused either by FRET $I_{F}$ or by direct excitation $I_{A}$ and the mean photon arrival time $E_{\mathrm{tA}}$ as coordinates for each single FRET pair. Over 150 individual FRET pairs of each FRET construct, detailed in table 15.1, were measured with several different STED intensities. The ellipses have a diameter of two s.d. of each distribution. . . . . . . . . . . . . . .

21.1 Overview of the analysis workflow of spectral shifts. . . . . . . . . . .

21.2 Top left: Absorption and emission spectra of Atto532, plotted over the spectral detection windows of APDs 2 to 5. Excitation was at 510nm as indicated by a vertical line, STED at $775 \mathrm{~nm}$ was not used for these measurements. Besides: Three exemplary fluorescence intensity time traces, each consisting of two plots. The upper plot shows the total photon count per detection channel, the lower plot depicts the relative intensity per detector. The $\mathrm{x}$-axis states the time in ms, corresponding to 400 frames of $5 \mathrm{~ms}$ duration each. . . . . . . . . . . . . . . . . . . . . . . . 121

21.3 Absorption and emission spectra of Star635P, plotted over the spectral detection windows of APDs 5 to 8 . Excitation at 552nm, STED at $775 \mathrm{~nm}$ not used for these measurements. Besides: Three exemplary fluorescence intensity time traces, each consisting of two plots. The upper plot shows the total photon count per detection channel, the lower plot depicts the relative intensity per detector. The $\mathrm{x}$-axis states the time in $\mathrm{ms}$, corresponding to 400 frames of $5 \mathrm{~ms}$ duration each. . . . . . . . . . . . . . . . . 123

21.4 K-means cluster analysis of the spectral intensity distribution of Atto532 (clusters 3 and 4 ) and its photoconversion products. The averaged fractional fluorescence intensity in each time trace segment is plotted in a 3D scatter plot. A 3D view and 2D projections are shown. Data points belonging to the same cluster are marked with the same color. The centroid of each cluster is depicted in red. . . . . . . . . . . . . . . . . . 126

21.5 K-means cluster analysis of the spectral intensity distribution of Star635P (cluster 6) and its photoconversion products. The averaged fractional fluorescence intensity in each time trace segment is plotted in a 3D scatter plot. A 3D view and 2D projections are shown. Data points belonging to the same cluster are marked with the same color. The centroid of each cluster is depicted in red. . . . . . . . . . . . . . . . . . . . . . 127

21.6 Absorption and emission spectra of Star635P, plotted over the spectral detection windows of APDs 4 to 7. Excitation at 552nm, STED at 775nm. 130 
21.7 K-means cluster analysis of the spectral intensity distribution of Star635P (cluster 5) and its photoconversion products. The averaged fractional fluorescence intensity in each time trace segment is plotted in a 3D scatter plot. Two 3D views of APDs 4-6 (top) and APDs 5-7 (bottom) and 2D projections are shown. Data points belonging to the same cluster are marked with the same color. The centroid of each cluster is depicted in red.132

21.8 Left: Intensity time traces of absolute photon count (upper subplot) and fractional intensity per detector (lower subplot) are plotted over consecutive frames for two separately scanned regions. The has a ROI size of $25 \mathrm{px}$, which translates to $250 \mathrm{~nm}$. Right: All image frames in the marked sections were added, the total intensity STED image (left) and 2D Gaussian fit model (right) of each section are shown. . . . . . . . . . . . . . . . . . 134

21.9 Chemical structure of Atto532, taken from [6], and of Star635P, adapted from [144]. Sites that might be susceptible to photoreactions are marked (suggested by Alexey Butkevich, personal communication, 12th Dec. 2017). Sites whose changes might result in shifts to shorter emission wavelengths are encircled in blue, those that might cause shifts to longer emission wavelengths in orange (small shift) and red (larger shift). Sites that are less likely to be attacked with unknown result are marked in gray. Suggested photoproducts are shown in supplementary Fig. 28.6. . . . . . . 137

22.1 Data of a DNA 80 FRET construct. Loss of FRET signal due to donor bleaching. Top left: Time trace of photon count in each detection channel over 50 image frames. Middle: Intensity images of 250nm edge length and lifetime histograms summed for each section of the time trace. Bottom: Table of fitted FWHM, fitted donor lifetime and FRET values $E_{\text {FRET }}$ and $R_{\text {FRET }}$. Photon count in the FRET channel is the sum of sensitized emission, donor bleedthrough and acceptor direct excitation. . . . . . . . . . . . . 142

22.2 Data of a DNA 79 FRET construct. Donor blueing in two steps reduces FRET signal. Donor bleaches, acceptor bleaches shortly afterwards. Note the changed shape of the last histogram of the FRET channel due to loss of FRET. Top left: Time trace of photon count in each detection channel over 50 image frames. Top right: Table of fitted FWHM, fitted donor lifetime and FRET values $E_{\mathrm{FRET}}$ calculated from lifetime and intensity ratio and $R_{\text {FRET }}$. Below: Intensity images of $250 \mathrm{~nm}$ edge length and lifetime histograms summed for each section of the time trace. Photon count in the FRET channel is the sum of sensitized emission, donor bleedthrough and acceptor direct excitation. . . . . . . . . . . . . . . . . . 143 
22.3 Data of a DNA 79 FRET construct. Donor blueing after first frame reduces FRET signal. Donor blinks off, thus interrupts FRET. Donor blinks on, with increased FRET signal. Donor blinks off again, then on, with yet increased FRET, due to red-shift. Acceptor bleaches, thus unquenches donor emission. Lastly donor bleaches. In the last histogram the increase of the donor lifetime is clearly visible. Top left: Time trace of photon count in each detection channel over 50 image frames. Top right: Table of fitted FWHM, fitted donor lifetime and FRET values $E_{\mathrm{FRET}}$ calculated from lifetime and intensity ratio and $R_{\text {FRET }}$. Below: Intensity images of $250 \mathrm{~nm}$ edge length and lifetime histograms summed for each section of the time trace. Photon count in the FRET channel is the sum of sensitized emission, donor bleedthrough and acceptor direct excitation. . . . . . . . . . . . 145

22.4 Data of a DNA 80 FRET construct. Donor blueing after second frame lowers FRET. Acceptor blueing, but lower FRET signal in this state. It is probably due to changed absorption, the QY seems similar. Then acceptor redding, FRET signal still constant. Donor bleaches, then acceptor. Later different donor molecule appears. Late photon emission due to FRET clearly visible in histograms. Top left: Time trace of photon count in each detection channel over 50 image frames. Top right: Table of fitted FWHM, fitted donor lifetime and FRET values $E_{\mathrm{FRET}}$ and $R_{\mathrm{FRET}}$. Below: Intensity images of 250nm edge length and lifetime histograms summed for each section of the time trace. Photon count in the FRET channel is the sum of sensitized emission, donor bleedthrough and acceptor direct excitation.

27.1 Schematic illustration of a laser beam being diffracted by an acoustic wave inside an AOM crystal. Taken from [15]. . . . . . . . . . . . . . . . 176

27.2 Schematic of the acoustic diffraction of incident light in an AOTF. Taken from $[21] \ldots \ldots \ldots \ldots \ldots$

27.3 Wavevector diagram for acousto-optic diffraction in a uniaxial crystal.

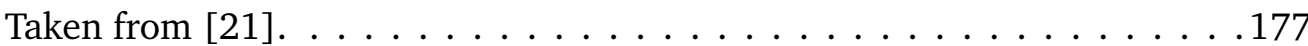

28.1 Top: Normalized fluorescence intensity of Star635 over consecutive frames. Fluorescence increase due to destruction of non-fluorescent dye aggregates by bleaching, then fluorescence decrease visible. Bottom: Normalized ratio of intensity detected on APDs 6 (blue) and 7 (red) remains largely constant, showing that spectral shifting was low. Intensity detected on APDs 3 and 4 was negligible. . . . . . . . . . . . . . . . . . . 180

28.2 Lifetime histograms accumulated in donor and FRET channel for each single molecule FRET construct with different STED intensity while keeping the pulse delay constant. . . . . . . . . . . . . . . . . . . . . 181 
28.3 Lifetime histograms accumulated in donor and FRET channel for each single molecule FRET construct. The STED pulse delay was increased in 320 ps steps while keeping the STED intensity constant. The legend states the delay value in a.u., a conversion to ns is given in table 14.1. The origin of the double peak in the lowest right figure is unclear, because the corresponding curve in the donor channel, which was acquired simultaneously, looks normal. . . . . . . . . . . . . . . . . . . . . . . . . . 182

28.4 Scheme of spectral corrections of the raw image in the FRET channel. Correction for excitation by the STED beam can be neglected if the dyes are chosen well. The correction factors $\mathrm{x}$ and $\mathrm{y}$ are determined from singly labeled reference samples. . . . . . . . . . . . . . . . . .

28.5 Scatter plots with fluorescence intensity ratio $R_{\text {FRET }}$ and mean photon arrival time $E_{\mathrm{tA}}$ as coordinates for each single FRET pair. Over 150 individual FRET pairs of each FRET construct were measured with several different STED pulse delays. The delay value stated in ns refers to the delay of the STED trigger with respect to the excitation trigger and is converted to a.u. in table 14.1. The ellipses have a diameter of two s.d. of each distribution. 184

28.6 Chemical structures of Atto532, Star635P and suggested photoproducts. The original structure of Atto532 was taken from [6], and that of Star635P from [144]. Possible photoproducts were suggested by Alexey Butkevich (personal communication, 12th December 2017). Structural changes resulting in an emission shift to shorter wavelengths are marked in blue. Structural changes that may cause an emission shift to longer wavelengths are marked in red (large shift) and orange (small shift). Groups that are less likely to be attacked and would result in an unknown spectral change are encircled in gray. . . . . . . . . . . . . . . .

28.7 The absorption spectrum of nonfluorescent quencher Atto612Q and emission spectra of Star580 and Star635P are shown in relation to the STED wavelength of $775 \mathrm{~nm} . \ldots \ldots \ldots \ldots \ldots$

30.1 This code performs first an overview scan and then a series of small confocal scans to study spectral shifting of single dyes. . . . . . . . . . . 202

30.2 This code performs first a confocal overview scan and then a series of small scans. At each ROI, a medium confocal scan is done to refine the centering of the ROI on the single molecule. Then, a smaller STED scan with many frames follows. .....................

30.3 This peak finder locates the positions of single molecules or FRET pairs in a confocal overview scan. The list of positions is then passed to one of the algorithms above to perform small scans at each position. . . . . . . . . 205

30.4 This function extracts the image stacks from Imspector measurement files for further processing and saving in Python. . . . . . . . . . . . . . 206 


\section{List of Tables}

10.1 FRET efficiencies, corresponding transfer rates and lifetimes of the donor fluorophore used in simulations. The FRET efficiencies are the same as expected for the DNA constructs used in later experiments, which are

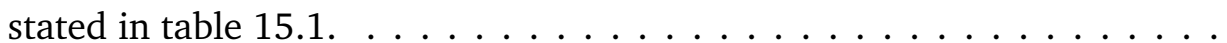

11.1 Transfer rates and decreased lifetimes of Cy3.5 were calculated assuming certain FRET efficiencies as in section 10.1 . . . . . . . . . . . . 63

14.1 STED pulse delay $\ldots \ldots \ldots \ldots \ldots \ldots \ldots \ldots$

15.1 Expected FRET efficiency $E_{\mathrm{FRET}}$ corresponding to different distances $\mathrm{r}$ of fluorophores on DNA. . . . . . . . . . . . . . .

17.1 Absorption and emission maxima, QY and fluorescence lifetime of tested green fluorophores according to the manufacturer. . . . . . . . . . .

17.2 Absorption and emission maxima, QY and fluorescence lifetime of tested red fluorophores according to the manufacturer. The QY of DyL633 was taken from $[90] . \ldots \ldots \ldots \ldots \ldots \ldots$

17.3 Bleaching rate $k_{\text {bleach }}$ of tested red fluorophores under 775nm STED illumination at two intensities. Standard error of the single exponential fit is given. . . . . . . . . . . . . . . . . . . . . 97

17.4 Excitation of tested red fluorophores by the 775nm STED beam at two intensities, relative to excitation with $612 \mathrm{~nm}$ at constant power. . . . . . . . 97

18.1 Single molecule FRET DNA constructs (described in table 15.1), their theoretically expected FRET efficiency $E_{\mathrm{FRET}}$ and corresponding lifetime $\tau_{\mathrm{DA}}$ (calculated in table 10.1) are stated. The fluorescence decay curves determined from confocal measurements (shown in Fig. 18.1) were fit with a single $\left(t_{1}\right)$ or double exponential decay fit (decay times $t_{1}, t_{2}$, amplitudes $A_{1}, A_{2}$ ) in Origin as necessary, the standard error (s.e.) of the fit is given. .101

21.1 Number of intensity traces of single Atto532 and Star635P molecules that either emit continuously with the same emission spectrum for the duration of the scan or that undergo at least one spectral shift towards the blue, the red or both consecutively. Atto532 was tested for photoconversion only in confocal mode, whereas Star635P was tested both confocally and with $775 \mathrm{~nm}$ STED. The measurements of Star635P with STED are described in detail in the following section 21.2 . . . . . . . . . . . . . . . 124 
21.2 Coordinates, i.e. relative intensities on the detectors, of the centroids of Atto532 clusters, therewith calculated spectral shifts, number of data points per cluster and average number of frames of the segments. . . . . . .127

21.3 Coordinates, i.e. relative intensities on the detectors, of the centroids of Star635P clusters, therewith calculated spectral shifts, number of data points per cluster and average number of frames of the segments. . . . . .

21.4 Spectral coordinates, i.e. fractional intensities on the detectors, of the centroids of Star635P clusters, and therewith calculated spectral shifts. Additionally, the number of data points $\mathrm{n}$ per cluster and average number of frames $t$ of the segments. Further, the average FWHM and s.d. of all sections (all frames added) in each cluster. Cluster 3 contains all scans where the fit of the FWHM did not converge. . . . . . . . . . . . . . .

22.1 Spectral shifts change FRET efficiency. . . . . . . . . . . . . . .

32.1 Samples for screening green and red dyes. Different structures in cultured Vero cells, namely nuclear pore complex protein Nup153 (Nup), tubulin (Tub) or mitochondrial protein Tom20 (Mito) were stained with dyelabeled antibodies. Stated are: the days on which the measurements were performed, excitation wavelengths, detectors, STED beam parameters and scan parameters. A gaussian-shaped STED focus was created by removing the phase plate from the beam path. In the "bleaching" column, it is stated which detection channels and frames were evaluated for obtaining the bleaching rate. In the "775nm" column the detection channels and frames which were used to calculate the excitation of the dye by the 775nm STED beam are listed. The number of times each line of the image was scanned, namely once with each excitation wavelength, is given in column "lines". The number of pixels in $\mathrm{x}$ and $\mathrm{y}$, the dwell time per pixel and the number of line steps were multiplied to obtain the duration of each frame. The scanner flyback was omitted because its duration is not known. . . . . . .

32.2 Experimental settings to test single dyes Atto532 and Star635P for photoconversion and to measure FRET between single pairs of Atto532 \& Star635P. Stated are: the days on which the measurements were performed, excitation wavelengths, detectors, STED parameters, scan settings, and the number of lifetime histogram bins. For the data set acquired in August 2017, spectral correction factors $x=0.19$ (D bleedthrough) and $y=0.16$ (A direct excitation) were measured with singly labeled DNA constructs. For the data set acquired in September 2017, $\mathrm{x}=0.21$ and $\mathrm{y}=0.16$ were obtained. . . . . . . . . . . . . . . . . . . . . . . . . . . . 214 



\section{Abstracts of publications}

\section{Publications}

Winter, F. R., Loidolt, M., Westphal, V., Butkevich, A. N., Gregor, C., Sahl, S. J., Hell, S. W. Multicolour nanoscopy of fixed and living cells with a single STED beam and hyperspectral detection. (2017). Scientific Reports 7:46492.

The extension of fluorescence nanoscopy to larger numbers of molecular species concurrently visualized by distinct markers is of great importance for advanced biological applications. To date, up to four markers had been distinguished in STED experiments featuring comparatively elaborate imaging schemes and optical setups, and exploiting various properties of the fluorophores. Here we present a simple yet versatile STED design for multicolour imaging below the diffraction limit. A hyperspectral detection arrangement (hyperSTED) collects the fluorescence in four spectral channels, allowing the separation of four markers with only one excitation wavelength and a single STED beam. Unmixing of the different marker signals based on the simultaneous readout of all channels is performed with a nonnegative matrix factorization algorithm. We illustrate the approach showing four-colour nanoscopy of fixed and living cellular samples.

Haralampiev, I., Prisner, S., Jolmes, F., Schade, M., Schreiber, M., Loidolt-Krüger, M., Jongen, K., Chamiolo, J., Winter, F., Seitz, O., Herrmann, A. Deciphering the assembly of multi-segment genome complexes in influenza A virus by the introduction of MuSeq-FISH. (2018). In preparation. 


\section{Posters}

Loidolt-Krüger, M., Westphal, V., Hell, S. W., STED Microscopy of FRET Pairs.

Poster presented at: Third Infinity 2017, 09.-11.10.2017, Göttingen

Förster Resonance Energy Transfer (FRET) is a popular tool in the life sciences, for example to detect protein-protein interactions or to construct fluorescent biosensors for small molecules. However, the fluorescence signal of several FRET pairs located within the detection volume is spatially averaged due to diffraction. This limits for example the dynamic range of biosensor readouts. We propose to use Stimulated Emission Depletion (STED) microscopy for locating and resolving distributions of FRET pairs more accurately and thereby reducing the spatial averaging of FRET signals. We performed numerical simulations of the excited state dynamics of a single FRET pair in order to visualize the combined photophysics of FRET and STED and to estimate the influence of different parameters. As a proof of principle experiment, we imaged and discriminated single FRET pairs attached to DNA at specific distances with FLIMSTED. Additionally, we used FLIM-STED to image the FRET signal between SNAP- and CLIP-tagged Gag proteins in HIV-1 particles before and after maturation of the viruses.

Loidolt, M., Winter, F. R., Haralampiev, I., Prisner, S., STED Microscopy of Influenza Virus Assembly.

Poster presented at: Focus on Microscopy 2017, 09.-12.04.2017, Bordeaux

Influenza A viruses cause seasonal epidemics and occasionally lead to pandemics. Vaccination is complicated by genetic reassortment between different virus strains that co-infect the same cell. Therefore, it is of great interest to understand how the viral genome is assorted and selectively packaged into newly forming virus particles. Multicolor STED microscopy enables the discrimination of otherwise overlapping signals, allowing accurate colocalization analysis. Here, we demonstrate the feasibility of using multicolor STED microscopy to simultaneously image several viral RNA segments together with other cellular structures of interest in infected cells.

Winter, F. R., Loidolt, M., Westphal, V., Hell, S. W., 4-Colour STED-Nanoscopy With a Single STED-Beam.

Poster presented at: Focus on Microscopy 2015, 29.03.-01.04.2015, Göttingen

Multicolour Imaging

Mechanisms and dynamic processes in living cells result from the interplay of different proteins and molecules. A detailed understanding of such processes requires the observation of several molecular species at high spatial resolution at the same time. The simplest way in fluorescence microscopy is to label different structures with fluorescent markers. These are distinguished by their emission and absorption spectra leading to an unambiguous relation between marker and structure. Therefore the employed microscopy method needs to be able to discriminate several markers via different detec- 
tion channels on the nanometer scale. For this purpose STED-microscopy (STimulated Emission Depletion) is an excellent tool. The doughnut-shaped STED-beam keeps molecules in the periphery of a Gaussian excitation beam in a dark state. The resulting focal spot from which the fluorescence signal is then detected is no longer subjected to Abbe's diffraction limit. Limitations of this method arise from the necessity of matching and optimizing the excitation and STED-wavelength pair together with the detection channels. This can only be achieved for a small number of markers simultaneously, hence restricting the versatility and the number of observable structures of interest.

Set-up and Data Analysis

Here we present a set-up where up to 8 different excitation wavelengths can be arbitrarily chosen. The detection spans a range of 150nm in 4 channels. Along with a single STED-beam this enables the observation and spectral discrimination of several markers at once on the nanometer scale. Preliminary results show that 4 markers can unambiguously be distinguished by their emission spectra. Thereby a resolution on the nm scale was reached for all structures. Results were obtained with only one excitation and one STED-beam. Raw Data is being analyzed with a Non Negative Matrix Factorization algorithm. The obtained results are then compared to conventional Linear Unmixing. 


\section{Oral contribution}

Nordwig, L., Loidolt-Krüger, M., Butkevich, A. N., Mitronova, G. Y., Leutenegger, M., Hell, S. W., Photolysis Of Water-Soluble Carbopyronine Fluorophores And PhotoConversion Into Other Fluorophores

Oral contribution at: 15th Conference on Methods and Applications in Fluorescence, 10.-13.09.2017, Brügge

In single-molecule localization experiments, we observed a complex behaviour of many fluorophores that might be caused by in-situ photo-conversion. Similar observations were made during multicolour STED microscopy experiments, where the spectral shifts hampered linear unmixing of multicolour images. While photobleaching of cyanines in aqueous solutions was investigated in detail [1], little is known on the photolysis of carbopyronine fluorophores in aqueous solutions. For these reasons we performed photobleaching experiments with the carbopyronine fluorophores 610CP and 580CP that were recently synthesised by Butkevich et al. in our group [2]. Recently multi-step photobleaching was shown for a non-water-soluble tris(diethylamino)- trioxatriangulenium dye in organic solvents [3]. To verify multi-step photobleaching of 610CP and $580 \mathrm{CP}$, we irradiated aqueous solutions of these fluorophores in a cuvette with laser light of $532 \mathrm{~nm}$ and $570 \mathrm{~nm}$ wavelength. The photolysis process was monitored by UVVIS absorption and emission spectroscopy and by high-pressure liquid chromatography (HPLC). A blue shift of $10 \mathrm{~nm}$ was observed in the absorption and emission spectra of the bulk solution. By separation of the intermediate products with HPLC we observed several fluorescent species with up to $40 \mathrm{~nm}$ shifted absorption and emission spectra. Some photolysis products absorbing in the visible region of the spectrum fluoresced. Some of these products were identified by comparison of the measured mass-to-charge ratio $(\mathrm{m} / \mathrm{z})$ in the liquid chromatography mass spectrometry (LC-MS) to the $\mathrm{m} / \mathrm{z}$ of the species involved in the expected photolysis pathways. One of the fluorescent products of 610CP was identified as 580CP. We show that the sequential photolysis of carbopyronine dye 610CP leads to loss of methyl groups and can photoconvert 610CP into 580CP. Further irradiation of 580CP leads to the formation of red- and blue-shifted fluorescent photoproducts. Thereby we show that photobleaching of 610CP involves the dealkylation of the carbopyronine core on the first step with subsequent reactions that finally result in non-fluorescent photoproducts. In addition to these bulk measurements, we performed spectrally resolved singlemolecule photobleaching experiments in aqueous solution in a confocal microscope (c.f. setup [4]). We observed spectral jumps (blueing and redding) of individual fluorophores during irradiation, which we correlated to the spectral characteristics of some of the previously identified intermediate fluorescent products to underpin our proposed reaction pathways on the level of single molecules. In this study we investigated the conversion of carbopyronine fluorophores upon irradiation with green and yellow light. The photobleaching experiment results can add to the understanding of key reaction pathways in aqueous solutions and therefore in biological samples. By inhibiting the photolysis of residues one may eliminate main pathways involved in the photo-conversion of common fluorophores and avoid issues 
in multicolour imaging and spectroscopic measurements due to blue- and/or redshifted intermediates. Our observations might lead to a new class/generation of fluorophores with increased photostability in aqueous solutions and in the presence of oxygen.

\section{Diploma thesis}

\section{Mehrfarben-STED-Mikroskopie}

In the context of this thesis a multicolor stimulated emission depletion (STED) fluorescence microscope was constructed, using a supercontinuum excitation laser and a STED laser with a wavelength of $775 \mathrm{~nm}$. The selection of one or several arbitrary excitation wavelengths from the supercontinuum spectrum with a freely adjustable intensity as well as line by line switching between them was implemented using acousto optical tunable filters (AOTF). In a first step it was shown in confocal mode that employing the principle of hyperspectral detection can facilitate the differentiation of fluorescent dyes whose emission maxima differ by $20 \mathrm{~nm}$ or more. The addition of excitation multiplexing allows the reduction of this value to ca. $10 \mathrm{~nm}$. Thereby the discrimination of three fluorescent markers based solely on their spectral properties was demonstrated using fluorescent beads as well as E. coli strains expressing different fluorescent proteins. Afterwards, the STED parameters were optimized using single-color fluorescent beads, and a lateral resolution of approximately $40 \mathrm{~nm}$ could be demonstrated. 



\section{Curriculum vitae}

\section{Personal details}

\section{Maria Loidolt-Krüger}

Birthday: 26th April 1989

Birthplace: Lutherstadt Wittenberg

Nationality: German

\section{Education}

since 10/2014 Doctorate in physics - Georg-August-Universität Göttingen

- work carried out at the Max Planck Institute for Biophysical Chemistry in Göttingen

- topic: STED microscopy of FRET pairs

- supervisor: Prof. Dr. Stefan W. Hell

2008-2013 Studies in Biophysics - Technical University Kaiserslautern

- degree: Diplom Biophysik (grade: 1.3)

- Diploma thesis: "Mehrfarben-STED-Mikroskopie" (grade: 1.0)

- supervisors: Prof. Dr. Stefan W. Hell, Jun. Prof. Dr. Sandro Keller

2011-2012 Erasmus exchange studies - University of Southern Denmark, Odense

- modules: Medical Neurobiology, Molecular Disease Diagnostics, Human Pathophysiology

- research project: "Aberrant U1snRNP binding as a molecular defect mechanism What are the rules?"

1999-2007 High school Johann-Gottfried-Seume Gymnasium, Vacha 
- degree: Abitur (grade: 1.1)

- advanced courses: mathematics, chemistry

\section{Achievements}

- Scholarship of the German Academic Scholarship Foundation

- Freshman scholarship of the Technical University Kaiserslautern

\section{Work experience}

2007-2008 English teacher - Foreign Language Kindergarten, Taixing

- Kindergarten English teacher at the Nanjing Normal University Affiliated Foreign Language Kindergarten in Taixing, Jiangsu province, China

since 2018 Application specialist - PicoQuant, Berlin

- technical sales, responsible for laser scanning microscopes and upgrade kits 


\section{Colophon}

This thesis was typeset with $\operatorname{LT}_{\mathrm{E}} \mathrm{X} 2 \varepsilon$. It uses the Clean Thesis style developed by Ricardo Langner. The design of the Clean Thesis style is inspired by user guide documents from Apple Inc.

Download the Clean Thesis style at http://cleanthesis.der-ric.de/. 